\title{
Elucidation of protein-protein interactions in mitochondria by cross-linking mass spectrometry
}

\author{
Dissertation \\ for the award of the degree \\ "Doctor rerum naturalium" \\ of the Georg-August-Universität Göttingen
}

\author{
within the doctoral program \\ "Biomolecules: Structure - Function - Dynamics" \\ of the Georg-August University School of Science (GAUSS)
}

submitted by

Andreas Linden

from Stolberg (Rhld.), Germany

Göttingen, 2020 


\section{Thesis Committee}

Prof. Dr. Henning Urlaub, Bioanalytical Mass Spectrometry Group, Max Planck Institute for Biophysical Chemistry and Clinical Chemistry, University Medical Center Göttingen

Prof. Dr. Jürgen Wienands, Cellular and Molecular Immunology, University Medical Center Göttingen

Dr. Alexander Stein, Membrane Protein Biochemistry, Max Planck Institute for Biophysical Chemistry

\section{Members of the Examination Board}

Referee: Prof. Dr. Henning Urlaub, Bioanalytical Mass Spectrometry Group, Max Planck Institute for Biophysical Chemistry and Clinical Chemistry, University Medical Center Göttingen

$2^{\text {nd }}$ Referee: Prof. Dr. Peter Rehling, Cellular Biochemistry, University Medical Center Göttingen

\section{Further members of the Examination Board}

Prof. Dr. Jürgen Wienands, Cellular and Molecular Immunology, University Medical Center Göttingen

Dr. Alexander Stein, Membrane Protein Biochemistry, Max Planck Institute for Biophysical Chemistry

Dr. Ricarda Richter-Dennerlein, Cellular Biochemistry, University Medical Center Göttingen

Prof. Dr. Stefan Jakobs, Structure and Dynamics of Mitochondria, Max Planck Institute for Biophysical Chemistry

Date of oral examination: 6 July 2020 




\section{Abstract}

Saccharomyces cerevisiae can generate energy in form of ATP either by fermenting or by respiring nutrition. This depends on the availability of fermentable or non-fermentable carbon sources. The respective energy metabolism of yeast cells affects protein abundances and protein-protein interactions (PPIs). This is also observed in respective mitochondria, organelles in eukaryotic cells that are key players in ATP production under respiratory conditions. Proteins and their abundances can be identified by mass spectrometry (MS). In combination with chemical protein-protein cross-linking, MS is also capable of identifying hundreds of protein-protein cross-links in a single sample (XL-MS). A cross-linker induces a covalent bond between reactive amino acids providing lowresolution information about their proximity to each other under native conditions. In this thesis, protein-protein cross-linking networks in mitochondria isolated from yeast grown either on glucose, a fermentable carbon source, or on glycerol, a non-fermentable carbon source, were elucidated by XL-MS. Mitochondria were treated with the non-cleavable cross-linker BS3 and cross-linked peptides were identified by separate database searches covering the 400 most abundant proteins for each condition. This approach resulted in 386 and 396 uniquely identified protein-protein cross-links in the glucose and the glycerol condition, respectively. Additionally, a quantitative analysis of residue-to-residue crosslinks between both conditions was performed by using an isotopically labeled cross-linker. However, differences in protein abundances induced by the carbon sources rather than PPI dynamics were the main driving force for the obtained significant changes. In mitochondria derived from yeast grown on glycerol-containing medium, the internal $\mathrm{NADH}$ :ubiquinone oxidoreductase Ndi1 located in the matrix of mitochondria was shown to participate in an $\mathrm{Ndi1}_{\mathrm{CII}} \mathrm{CIV}_{2}$ electron transport chain supercomplex. Also, hitherto undescribed interactions and interactions of uncharacterized proteins were identified. Amongst them, the association of Min8 to the cytochrome $c$ oxidase was revealed. Biochemical experiments further corroborated this interaction and could show that Min8 might be involved in the assembly of Cox12 into an intermediate complex of cytochrome $c$ oxidase. The established cross-linking workflow was also applied to mitoplasts generated from mitochondria of human HEK cells. Here, it could be demonstrated that the use of cross-linkers with different reaction chemistries improves the results by providing complementary protein-protein cross-links. 


\section{Abbreviations}

$A B C$

ammonium bicarbonate

ACN

acetonitrile

AGC

automatic gain control

ambig

ambiguous

$\mathrm{BN}$

blue native

BS3

bis(sulfosuccinimidyl)suberate

BSA

bovine serum albumin

$\mathrm{Cl}-\mathrm{CV}$

complexes I-V of ETC

CID

collision-induced dissociation

cryo-EM

cryogenic-electron microscopy

CSM

cross-linked peptides spectrum match

DDA

data-dependent acquisition

DDM

n-dodecyl $\beta$-D-maltoside

DIA

data-independent acquisition

DMSO

dimethyl sulfoxide

DSS

disuccinimidyl suberate

DTT

dithiothreitol

EDC

1-ethyl-3-(3-dimethylaminopropyl)carbodiimide hydrochloride

ESI

electrospray ionization

ETC

electron transport chain

FDR

false discovery rate

$\mathrm{HCD}$

higher-energy collisional dissociation

IAA

iodoacetamide

iBAQ

intensity-based absolute quantification

IM

inner membrane

IMS

intermembrane space

IP

immunoprecipitation

IT

injection time

LC-MS/MS

liquid chromatography tandem mass spectrometry

$m / z$

mass-to-charge ratio

MIA

mitochondrial intermembrane space import and assembly

MICOS

mitochondrial contact-site and cristae-organizing system

mitoribosome

mitochondrial ribosome

mtDNA

mitochondrial DNA

$\mathrm{NHS}$

$\mathrm{N}$-hydroxysuccinimide 


\begin{tabular}{|c|c|}
\hline not mt & not mitochondrial \\
\hline $\mathrm{OM}$ & outer membrane \\
\hline ORF & open reading frame \\
\hline OT & orbitrap \\
\hline OXPHOS & oxidative phosphorylation \\
\hline PAGE & polyacrylamide gel electrophoresis \\
\hline PAM & presequence translocase-associated motor \\
\hline PCA & principle component analysis \\
\hline Pdc & pyruvate decarboxylate \\
\hline $\mathrm{PDH}$ & pyruvate dehydrogenase complex \\
\hline PK & proteinase $\mathrm{K}$ \\
\hline PPI & protein-protein interaction \\
\hline ppm & parts per million \\
\hline PSM & peptide spectrum match \\
\hline PTM & post-translational modification \\
\hline PTP & permeability transition pore \\
\hline qXL-MS & quantitative XL-MS \\
\hline Rcf & respiratory supercomplex factor \\
\hline ROS & reactive oxygen species \\
\hline RT & room temperature \\
\hline SCX & strong cation exchange \\
\hline SDS & sodium dodecyl suflate \\
\hline SEC & size exclusion chromatography \\
\hline SGD & Saccharomyces Genome Database \\
\hline SILAC & stable isotope labeling by amino acids in cell culture \\
\hline TCA & tricarboxylic acid cycle \\
\hline TFA & trifluoroacetic acid \\
\hline TMT & tandem mass tags \\
\hline TOM/TIM & translocase of outer and inner membranes \\
\hline VDAC & voltage-dependent anion channel \\
\hline WT & wild type \\
\hline XL-MS & cross-linking mass spectrometry \\
\hline
\end{tabular}




\section{List of figures}

Figure 1: Morphology and functions of a mitochondrion. ....................................... 2

Figure 2: Cytochrome $c$ oxidase assembly in yeast mitochondria. ............................. 9

Figure 3: Classical shotgun proteomics workflow. ............................................. 12

Figure 4: Reaction schema of protein-protein cross-linking with BS3. ...................... 16

Figure 5: Comparison of both cross-linkers DSS and BS3 regarding their ability to crosslink proteins in all mitochondrial subcompartments.

Figure 6: Cross-linking workflow for the identification of cross-linked peptides in mitochondria isolated from Saccharomyces cerevisiae.

Figure 7: Relative distribution of protein-protein cross-links after cross-linking of mitochondria derived from yeast grown on glycerol- or glucose-containing medium according to the proteins' subcompartmental localizations.

Figure 8: Global interprotein cross-linking network identified in mitochondria derived from yeast grown on glycerol- or glucose-containing medium.

Figure 9: Cross-links of selected proteins as identified in mitochondria isolated from yeast grown on glycerol- or glucose-containing medium.

Figure 10: Interprotein cross-links within the OXPHOS system identified in mitochondria derived from yeast grown either under glycerol or glucose condition.

Figure 11: Results from quantitation of unique residue-to-residue cross-links after crosslinking mitochondria derived from yeast grown on glycerol- or glucose-containing medium with isotopically labeled and unlabeled BS3.

Figure 12: Representation of intraprotein and interprotein cross-links that were quantified after cross-linking mitochondria derived from yeast grown on glycerol- or glucosecontaining medium with isotopically labeled and unlabeled BS3.

Figure 13: Ratios of copy numbers of quantified cross-linked proteins in $S$. cerevisiae grown under glycerol and glucose condition.

Figure 14: Cross-links within the pyruvate dehydrogenase complex $(\mathrm{PDH})$ identified in mitochondria derived from yeast grown on glycerol- or glucose-containing medium. .... 52 Figure 15: $\mathrm{CIII}_{2} \mathrm{CIV}_{2}$ supercomplex structure with calculated accessible interaction space of Ndi1. 54

Figure 16: Proposed structure of an $\mathrm{Ndi1}_{1} \mathrm{ClII}_{2} \mathrm{CIV}_{2}$ supercomplex in yeast. 55

Figure 17: Proposed structural model of Min8 associated with the cytochrome $c$ oxidase.

Figure 18: Association of Min8 to the cytochrome $c$ oxidase. 
Figure 19: Relative distribution of protein-protein cross-links after cross-linking mitochondria derived from the $r \mathrm{o}^{\circ}$ yeast strain according to their subcompartmental localization.

Figure 20: Cross-links within the OXPHOS system and the pyruvate dehydrogenase complex $(\mathrm{PDH})$ identified in mitochondria derived from the $r \mathrm{o}^{\circ}$ yeast strain. 60

Figure 21: Identified cross-links within the TCA cycle and its connection to proteins involved in amino acid biosynthesis in mitochondria derived from $r h o^{\circ}$ yeast cells. 61

Figure 22: Cross-linking network of TOM/TIM proteins identified in mitochondria derived from $r h 0^{0}$ yeast cells as well as from wild type mitochondria derived from yeast grown on glycerol- or glucose-containing medium.

Figure 23: Selected protein-protein cross-links uniquely identified in the protein SEC approach after cross-linking mitochondria derived from yeast grown on glycerol-containing medium.

Figure 24: Principal component analysis (PCA) of glycerol, glucose and $r 0^{\circ}$ condition. . 66

Figure 25: Oxygen flux of isolated and purified yeast mitochondria measured with Oroboros. 68

Figure 26: Import assay. 69

Figure 27: Activity staining of CII, CIV and CV after BN-PAGE. 70

Figure 28: Proteinase $\mathrm{K}$ assay of frozen purified mitochondria. 71

Figure 29: Relative distribution of isoelectric points ( $\mathrm{pl}$ ) of all proteins involved in crosslinking reactions with either BS3 or EDC in percent after cross-linking human mitoplasts.

Figure 30: Global protein-protein cross-linking network in mitoplasts generated from human mitochondria. 76

Figure 31: Human $\mathrm{CICIII}_{2}$ supercomplex structure. 77 


\section{List of tables}

Table 1: List of used chemicals and suppliers.................................................... 21

Table 2: List of used buffers and their compositions............................................. 22

Table 3: List of commonly used consumables and suppliers. .................................. 23

Table 4: List of regularly used equipment and suppliers. ....................................... 23

Table 5: List of used software with indicated version and developers. .......................... 24

Table 6: Numbers of total identified cross-linked peptides spectrum matches (CSMs), cross-linked proteins, unique residue-to-residue cross-links and the interprotein crosslinks in percent for the unfiltered and the filtered data set for all conditions. 42

Table 7: Numbers of total identified cross-linked peptides spectrum matches (CSMs), cross-linked proteins, unique residue-to-residue cross-links and the interprotein crosslinks in percent for the unfiltered and the filtered data set for peptide SEC and the protein SEC approach.

Table 8: Protein-protein cross-links within the OXPHOS system of mitochondria derived from yeast grown on glycerol-containing medium uniquely identified via the protein SEC approach.

Table 9: Numbers of total identified cross-linked peptides spectrum matches (CSMs), cross-linked proteins, unique residue-to-residue cross-links and the interprotein crosslinks in percent for the unfiltered and the filtered data set after BS3- or EDC-cross-linking of human mitoplasts.

Table 10: Proteins involved in BS3- and EDC-induced cross-linking reactions ranked according to their numbers of cross-linked peptides spectrum matches (CSMs) contributing to all identified CSMs. 75

Table 11: Comparison between so far published studies dealing with XL-MS of mitochondria. 102 


\section{Table of contents}

Abstract $\begin{array}{ll}\text { Abbreviations } \\ \text { List of figures } \\ \text { List of tables } \\ \text { Table of contents }\end{array}$

1.1. Saccharomyces cerevisiae and its role as model organism........................... 1

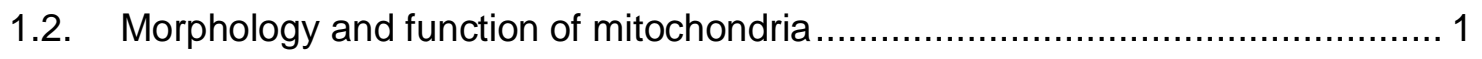

1.3. Genome maintenance in mitochondria .......................................................... 3

1.4. Protein import into mitochondria ......................................................... 4

1.5. Energy metabolism of $S$. cerevisiae and the role of mitochondria...................... 5

1.6. The oxidative phosphorylation system................................................... 6

1.6.1. Organization of the OXPHOS complexes ........................................... 6

1.6.2. Biological significance for supercomplex formation ................................ 7

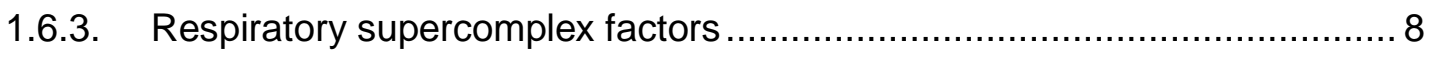

1.6.4. Assembly of the cytochrome $c$ oxidase ........................................... 8

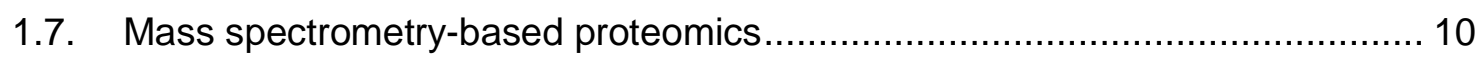

1.7.1. General setup and principle of MS-based proteomics ......................... 10

1.7.2. Protein identification..................................................................... 12

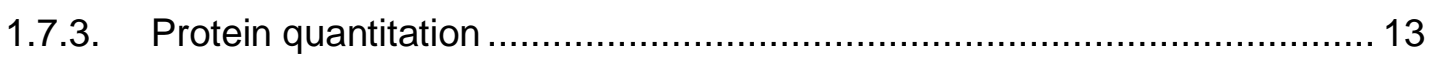

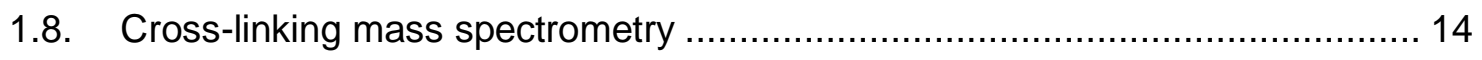

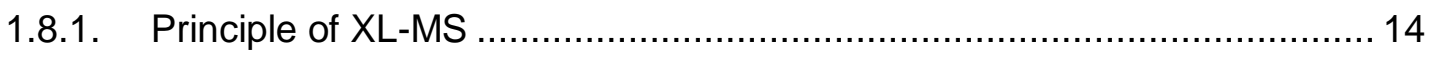

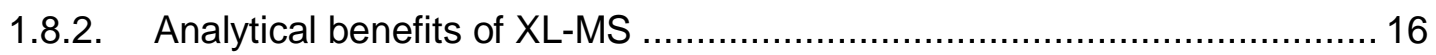

1.8.3. Adapting the proteomics workflow for XL-MS ................................... 17

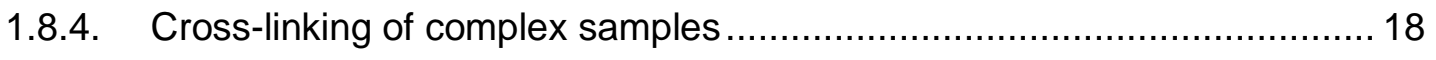

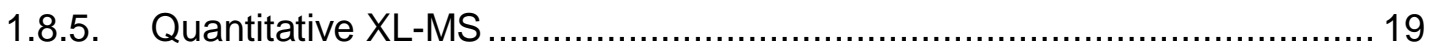

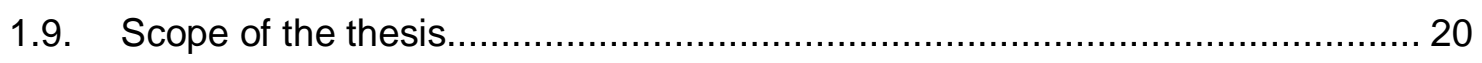

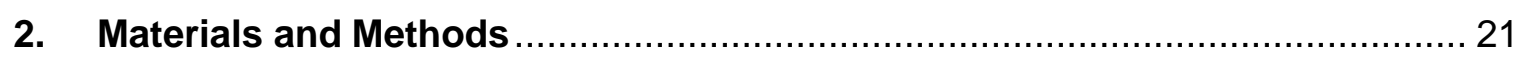

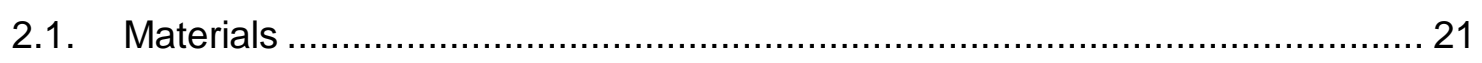

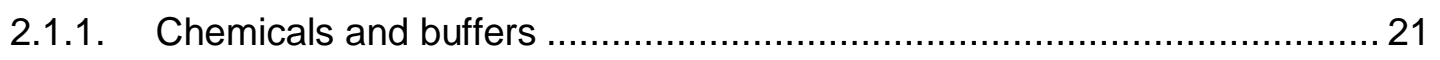

2.1.2. Equipment and consumables ..................................................... 23 


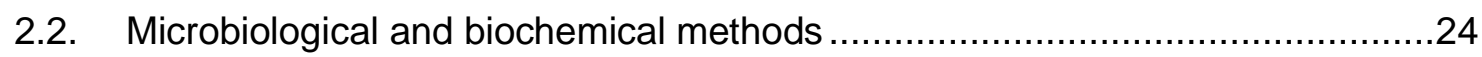

2.2.1. Saccharomyces cerevisiae growth conditions .................................... 24

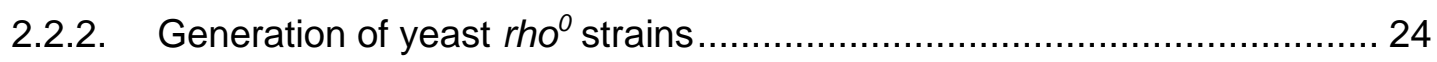

2.2.3. Isolation of mitochondria from S. cerevisiae......................................... 25

2.2.4. Tandem sucrose gradient centrifugation ........................................ 25

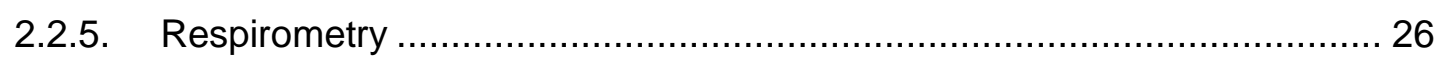

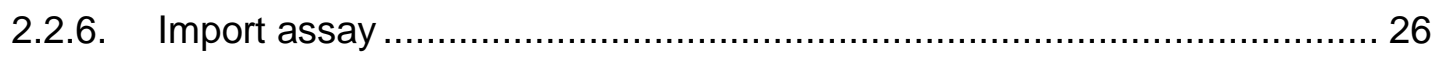

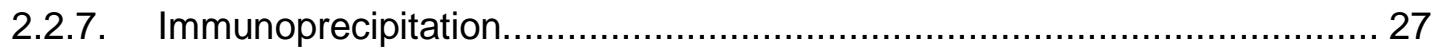

2.2.8. Blue native-PAGE and activity staining .............................................. 27

2.2.9. Western blotting and immunodetection .............................................. 28

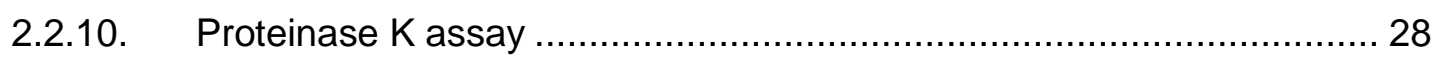

2.2.11. HEK293T cell culture ........................................................... 29

2.2.12. Isolation of human mitochondria and generation of mitoplasts ............ 29

2.2.13. Cross-linking of purified yeast mitochondria, lysis and protein precipitation

2.2.14. Cross-linking of human mitoplasts, lysis and protein precipitation ....... 30

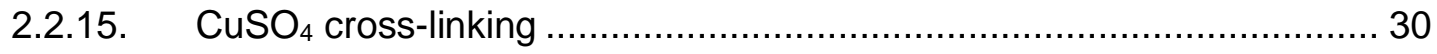

2.2.16. Protein size exclusion chromatography ......................................... 31

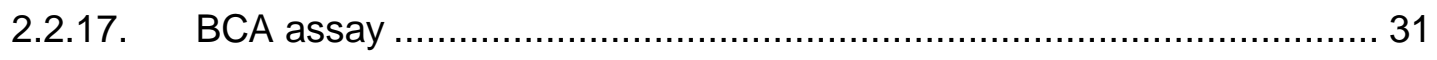

2.3. Mass spectrometry and proteomics methods ...........................................

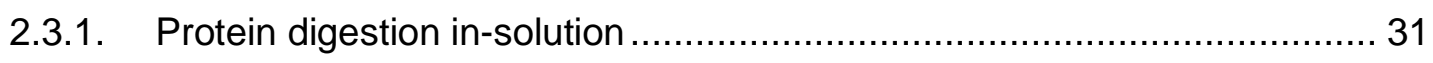

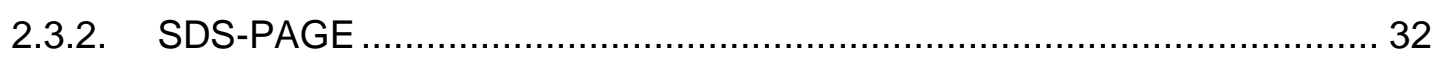

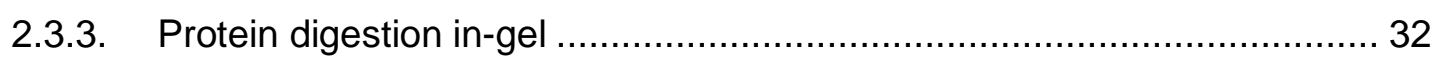

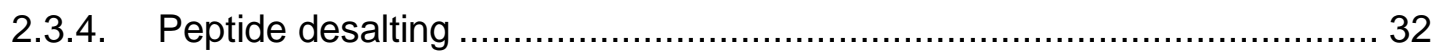

2.3.5. Enrichment of cross-linked peptides by size exclusion chromatography .. 33

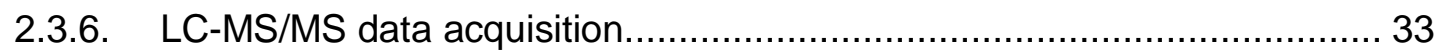

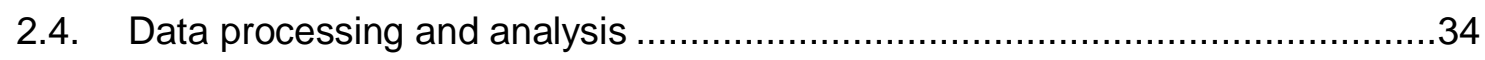

2.4.1. Analysis of data acquired from cross-linked yeast mitochondria.............. 34

2.4.2. Analysis of data acquired from cross-linked human mitoplasts ............... 35

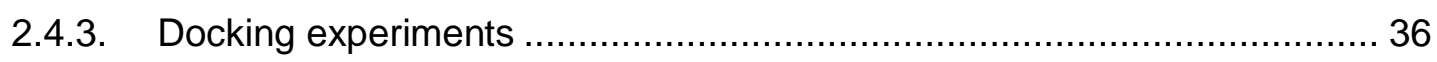

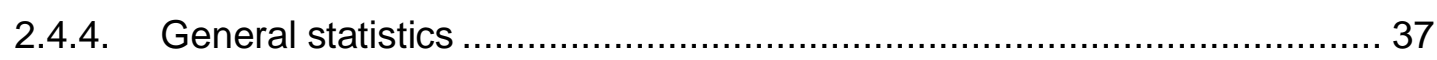

2.4.5. Visualization of cross-linking results.............................................. 37 
3.1. Cross-linking combined with mass spectrometry of mitochondria derived from yeast grown on glycerol- or glucose-containing medium.

3.1.1. Determination of a suitable cross-linker to cross-link mitochondrial proteins .39

3.1.2. Workflow for cross-linking mitochondria 40

3.1.3. Cross-links reveal different protein-protein cross-linking patterns in yeast mitochondria depending on the carbon source 43

3.1.4. Cross-links between proteins localized to the inner membrane ................ 45

3.1.5. Cross-links within the OXPHOS system ................................................ 47

3.1.6. Quantitative XL-MS approach reveals abundance changes of residue-toresidue cross-links depending on the carbon source ...................................... 48

3.1.7. Organization of the pyruvate dehydrogenase complex.......................... 51

3.1.8. Interactions of uncharacterized proteins ............................................. 52

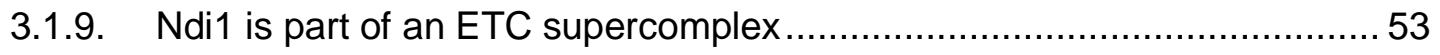

3.1.10. Min8 is associated with the cytochrome $c$ oxidase ..............................55

3.2. Cross-linking combined with mass spectrometry of mitochondria derived from a

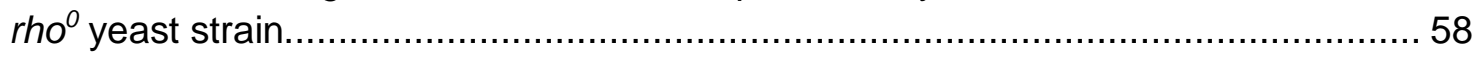

3.2.1. Cross-links within the OXPHOS system and the PDH..........................59

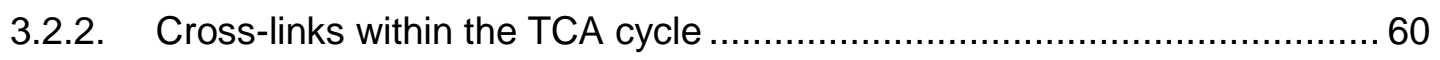

3.2.3. Cross-links within TOM/TIM complexes ........................................... 61

3.3. Protein size exclusion chromatography as additional fractionation step to improve

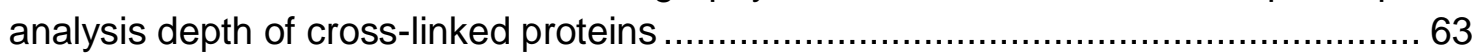
3.4. Proteomic analysis of mitochondria derived from yeast grown on glycerol- or glucose-containing medium and from the $r h 0^{0}$ yeast strain ......................................6

3.5. Quality control of yeast mitochondria........................................................ 67

3.5.1. Oxygen consumption of isolated and purified yeast mitochondria ............67 67

3.5.2. Import assay with isolated and purified yeast mitochondria ..................... 69

3.5.3. Activity assays to investigate functional integrity of ETC complexes ........70

3.5.4. Proteinase $\mathrm{K}$ assay to investigate mitochondrial membrane integrity ........71

3.6. Cross-linking combined with mass spectrometry of mitoplasts derived from

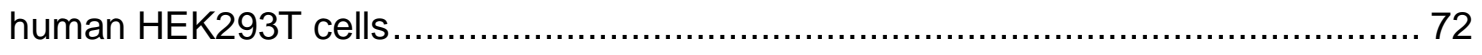

3.6.1. BS3 and EDC cross-link different regions of proteins ........................... 73

3.6.2. Intraprotein cross-links outnumber interprotein cross-links...................... 74 
4. Discussion

4.1. Integrity of mitochondrial membranes and its impact on structural analysis .....78 4.2. BS3 and its ability to cross-link proteins of all mitochondrial subcompartments 79 4.3. Benefits and drawbacks of restricted databases for cross-linked peptides searches .81

4.4. Protein-protein cross-links in mitochondria derived from yeast grown on either glycerol- or glucose-containing medium

4.4.1. A general comment on the filtering of the XL-MS data sets 82

4.4.2. Majority of cross-linked proteins localized to the inner membrane or matrix 83

4.4.3. Differences in the protein-protein cross-linking patterns between mitochondria derived from yeast grown on either glycerol- or glucose-containing medium .85

4.4.4. The oxidative phosphorylation system 86

4.4.5. The pyruvate dehydrogenase complex 92

4.4.6. Interactions of uncharacterized proteins. 95

4.4.7. Protein size exclusion chromatography slightly improved analysis depth. 98 4.4.8. A technical comparison with other studies dealing with XL-MS of mitochondria... .99

4.5. Protein-protein cross-links in mitochondria derived from a $r h o^{\circ}$ yeast strain $\ldots 103$ 4.5.1. Energy metabolism and amino acid biosynthesis pathways - or a dual role for mitochondrial proteins? ........................................................................ 103

4.5.2. TOM/TIM complexes................................................................ 104

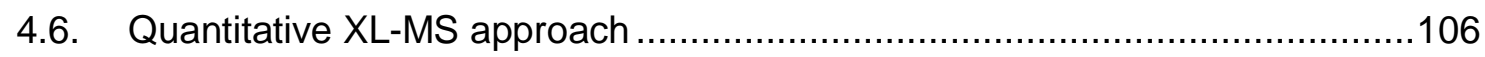

4.6.1. Challenges of quantitative XL-MS ............................................. 107

4.6.2. Biological interpretation of the quantitative data ................................ 108

4.6.3. Other labeling strategies in combination with XL-MS ........................ 110

4.7. Cross-linking of human mitoplasts with two different cross-linkers ................111

4.7.1. General comments on the experimental rationale ............................... 111

4.7.2. Cross-linkers with different reaction chemistries ................................ 112

4.7.3. Intraprotein cross-links outnumbered interprotein cross-links................ 113

4.7.4. Biological interpretation of the identified protein-protein cross-links ....... 114 
4.8. Other cross-linking and analysis workflows

4.8.1. Enrichment of cross-linked peptides to increase their identification rate. 116

4.8.2. Reducing the search space

4.9. Other strategies for interrogating protein-protein interactions using mass spectrometry ....

4.10. Summary and perspective

120

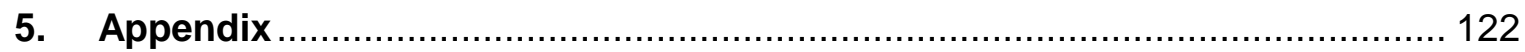

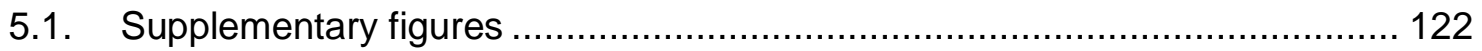

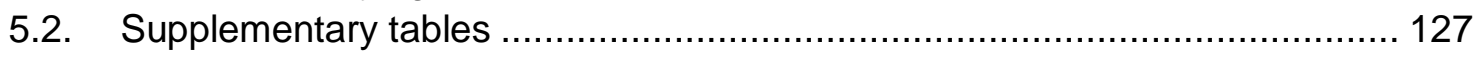

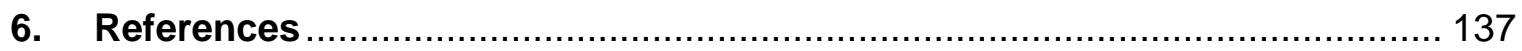

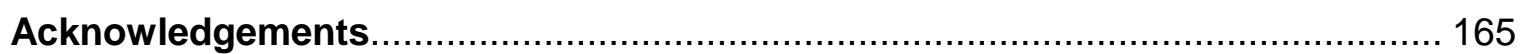

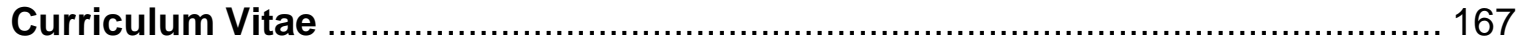







\section{Introduction}

\subsection{Saccharomyces cerevisiae and its role as model organism}

The baker's yeast Saccharomyces cerevisiae is a prime example of a eukaryotic model organism used in scientific research. Albeit being one of the simplest eukaryotic organisms, it shares many of the essential cellular processes with humans. This accompanied by a fully sequenced genome and the ease of manipulation ${ }^{1}$ has made yeast instrumental to study gene expression regulation ${ }^{2}$, metabolism $^{3}$, aging ${ }^{4}$, apoptosis ${ }^{5}$ and neurodegenerative diseases ${ }^{6}$, amongst others. Particularly in the study of human diseases and the transfer of significant results to Homo sapiens, the use of yeast as model organism seems reasonable as $30 \%$ of genes involved in human diseases are estimated to have orthologs in yeast ${ }^{7}$. S. cerevisiae, also known as budding yeast, can be cultured under various conditions in laboratories and by its short generation time it produces sufficient material for biochemical research in a cost- and time-efficient manner ${ }^{1}$. The fully sequenced genome not only paved the way for genetic engineering, but also to unravel the whole proteome, counting a total of 6049 proteins (according to the UniProt reference proteome of Saccharomyces cerevisiae, May 2020). This, in turn, encouraged researchers to address scientific questions that could not be answered by genetic analyses alone. Post-translational modifications (PTMs) of proteins $^{8}$, their abundances ${ }^{9,10}$ and their interactions to other proteins ${ }^{11}$ play an important role in the investigation of biological processes. S. cerevisiae emerged as an optimal model organism specifically for researching the biological functions and biogenesis of mitochondria ${ }^{1}$, organelles that perform tasks that are tremendously important for cell survival' ${ }^{12}$. Dysfunctional mitochondria are linked to diseases such as Leigh and Barth syndromes ${ }^{13}$ but also to neurodegenerative diseases such as Parkinson's or Alzheimer's diseases ${ }^{14}$, giving impetus for research on mitochondria.

\subsection{Morphology and function of mitochondria}

Mitochondria are commonly referred to as "powerhouses" in eukaryotic cells generating ATP, the major energy source. Originated from $\alpha$-proteobacteria ${ }^{15}$, mitochondria are organelles consisting of two membranes, the outer membrane (OM) and the inner membrane (IM). Consequently, this leads to the formation of two soluble compartments, the intermembrane space (IMS) located between both membranes and the matrix, enclosed by the IM. The IM has a much larger surface than the OM due to the formation of cristae protruding into the matrix (Figure 1). This shape is mainly affected by the 
mitochondrial contact-site and cristae-organizing system ${ }^{16,17}$ (MICOS) and the $F_{1} F_{0}$ ATP synthase that forms a V-shaped dimeric structure ${ }^{18-20}$. The $F_{1} F_{0}$ ATP synthase is also one of the key players in energy production. The IM harbors all complexes of the electron transport chain (ETC) - four in mammals and three in yeast - representing a huge redox reaction network. The ETC together with the $F_{1} F_{0}$ ATP synthase form the oxidative phosphorylation (OXPHOS) system covering the main ATP demand of a cell. In the early days, it was the focus of mitochondrial research to unravel the mystery behind ATP production. In the last two decades, it became more and more clear that mitochondria are not only involved in bioenergetics, but also in other important cell processes such as the metabolism of amino acids and lipids ${ }^{21}$, providing iron-sulfur $(\mathrm{Fe} / \mathrm{S})$ clusters $^{22}$ or triggering apoptosis $^{23}$, amongst others (Figure 1). Mitochondria possess their own genome (mitochondrial DNA, mtDNA) encoding two rRNAs, tRNAs and eight proteins in yeast ${ }^{24}$ and 13 proteins in mammals ${ }^{25}$, accompanied by their own mitochondrial ribosome (mitoribosome) 26-28.

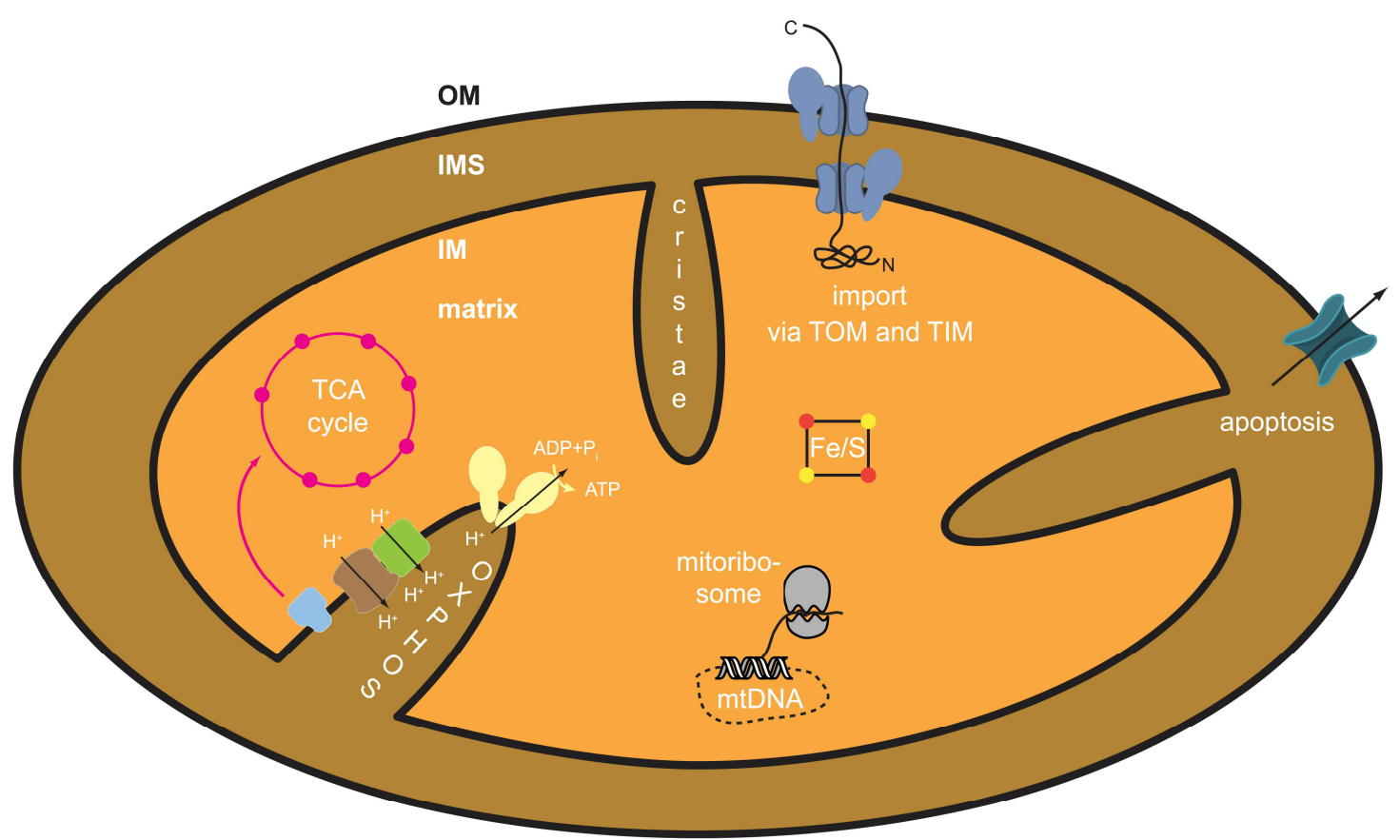

Figure 1: Morphology and functions of a mitochondrion. Mitochondria are organelles with four subcompartments: the outer membrane (OM), the intermembrane space (IMS), the inner membrane (IM) and the matrix. The IM forms invaginations called cristae. Depicted are selected key functions such as the oxidative phosphorylation (OXPHOS) system including complexes II-IV (in yeast) and the $\mathrm{F}_{1} \mathrm{~F}_{0}$ ATP synthase dimer for ATP production, the tricarboxylic acid (TCA) cycle for generating redox equivalents, the mitochondrial DNA (mtDNA), the mitochondrial ribosome (mitoribosome), iron-sulfur (Fe/S) cluster generation, protein import via the translocase of the outer and inner membrane (TOM/TIM) and apoptosis (in mammals). Not drawn to scale and without claiming completeness. Adapted by permission from Pfanner, Warscheid and Wiedemann ${ }^{21}$. 
With all the tasks mitochondria have to fulfill it immediately becomes clear that more proteins are necessary. The mitochondrial proteome consists of approximately 1000 proteins in yeast ${ }^{29}$ and 1500 proteins in mammals ${ }^{30}$. Consequently, $99 \%$ of mitochondrial proteins are encoded by genes located in the nucleus. These proteins have to be imported into and translocated by mitochondria. This is accomplished by translocases of the outer and the inner membranes (TOM and TIM) ${ }^{31,32}$. All these functions keep a cell alive and are achieved by plenty of mitochondria, forming a dynamic network promoted by constant fusion and fission events ${ }^{33}$. This network underlies changes depending on cell types, the cell's energy demand and availability of nutritions ${ }^{34}$. Mitochondria in $S$. cerevisiae grown on fermentable carbon sources show unbranched and short networks, while a nonfermentable carbon source induces large branched networks ${ }^{35}$.

\subsection{Genome maintenance in mitochondria}

Mitochondrial DNA (mtDNA) is approximately 70-85 kilobase pairs long in S. cerevisiae ${ }^{36}$. It encodes two rRNAs, all tRNAs and eight proteins in yeast and 13 proteins in mammals comprising hydrophobic core subunits of complexes within the respiratory chain and subunits of the $F_{1} F_{0}$ ATP synthase ${ }^{37,38}$. At least for growth in non-fermentable carbon sources an intact mtDNA is, thus, indispensable for cell survival. Like in other compartments of the cell that contain DNA, mitochondria harbor machineries for DNA packaging, transcription, translation and repairing. The mtDNA is wrapped around DNAbinding proteins to form nucleoids ${ }^{39}$. In yeast, the main protein for packaging is $A b f 2^{40}$. The mitochondrial RNA polymerase Rpo4 $1^{41,42}$ transcribes the DNA to RNA in yeast, which is finally translated into proteins by the mitochondrial ribosome ${ }^{26,27}$. In case of DNA damage, not only general mechanisms such as base excision and mismatch repair, amongst others, have been reported to occur, but also mitochondria-specific events ${ }^{43,44}$. Surprisingly, maintenance and stability of mtDNA is supported by proteins with a dual role such as proteins usually involved in energy metabolism ${ }^{45}$ or amino acid biosynthesis ${ }^{46}$. This was investigated by the knockout of respective genes in yeast strains with subsequent screening and testing for respiratory-deficient mutants, so-called petite mutants. These mutants either contain severely damaged mtDNA ( $r o^{-}$strains) or completely lack mtDNA ( $r h o^{\circ}$ strains). Since these strains cannot synthesize core proteins of the respiratory system, they can only grow on fermentable media ${ }^{47}$. During evolution, mtDNA has lost most of its genes due to redundancy with nuclear genes or their transfer to the nucleus ${ }^{48}$. Consequently, $99 \%$ of all mitochondrial proteins are encoded by nuclear DNA, and protein import into mitochondria is essential for their functionality and cell viability ${ }^{32}$. 


\subsection{Protein import into mitochondria}

More than a thousand of mitochondrial proteins are translated in the cytosol and have to be imported into and translocated by mitochondria. Most of those proteins carry mitochondrial targeting signals located within the protein sequence or at the N-terminus, positively charged and forming an amphipathic helix, depending on their final destination ${ }^{49,50}$. Regardless of their mitochondrial localization, the translocase of the outer membrane (TOM) complex is the main entry gate for the majority of the proteins ${ }^{51}$. Small receptor subunits protruding to the cytosol interact with still unfolded precursor proteins and guide them through a $\beta$-barrel shaped pore ${ }^{52}$. Consecutively, distinct sorting pathways exist that lead the proteins to their target compartment. $\beta$-barrel shaped proteins of the OM are sorted by the sorting and assembly machinery (SAM) complex ${ }^{53,54}$ in combination with small translocase of the inner membrane (TIM) proteins ${ }^{55}$, while $\alpha$-helical proteins are translocated by the OM protein Mim1 ${ }^{56,57}$. Proteins whose final destination is the IMS are further processed by the mitochondrial intermembrane space import and assembly (MIA) machinery consisting of the receptor Mia40 and the sulfhydryl oxidase Erv1 ${ }^{58}$. Soluble proteins possessing a cysteine-rich sequence motif are oxidatively folded by a disulfide relay system that forms disulfide bridges within these proteins. Translocation of proteins to the IM is mediated by the TIM22 and TIM23 complexes ${ }^{51}$. Precursor proteins entering the IMS through the TOM complex are chaperoned by small TIMs and guided to the TIM22 complex ${ }^{59}$. After docking to a receptor protein, the precursor protein is forwarded to the TIM22 pore. In a membrane potential $(\Delta \Psi)$-dependent manner, proteins are laterally released into the lipid bilayer ${ }^{60-62}$. Major substrates of this pathway are metabolite carriers $^{51,63}$ such as the ADP/ATP carrier protein (Pet9). Proteins with an N-terminal mitochondrial targeting signal are mainly substrates of the TIM23 complex ${ }^{64,65}$. After passing through the TOM pore, receptor subunits of the TIM23 complex direct the precursor protein to its pore forming subunit. To achieve this, both the TOM and the TIM23 complexes physically interact with each other, creating a TOM-TIM23 supercomplex ${ }^{66,67}$. Depending on the protein's sequence it is either laterally released into the IM driven by $\Delta \Psi^{68,69}$ or channeled to the matrix. Matrix translocation is fueled not only by $\Delta \Psi$ but can also be supported by the presequence translocase-associated import motor (PAM) complex ${ }^{70,71}$. This complex associates with the TIM23 complex on the matrix-side, and, driven by ATP hydrolysis, ultimately guides proteins to the matrix. Mitochondrial targeting signals are finally digested by the mitochondrial processing peptidase (MPP) leading to mature proteins ${ }^{49}$. Copy numbers of proteins belonging to TOM or TIM complexes are comparably stable amongst different growth conditions and are barely affected by the cell's energy demand ${ }^{21,72}$. 


\subsection{Energy metabolism of $S$. cerevisiae and the role of mitochondria}

In the presence of oxygen, Saccharomyces cerevisiae can generate ATP either by fermentation or by respiration, strongly depending on the available nutrition, the carbon source. The preferred carbon source of $S$. cerevisiae is glucose, a fermentable carbon source. Although oxygen is present, in high concentrations of glucose the cells start to produce energy by fermentation. This is the so-called Crabtree effect ${ }^{73}$. Here, yeast cells process glucose via the glycolytic pathway to pyruvate. Instead of fully respirate the pyruvate via the oxidative phosphorylation system located in the mitochondria, pyruvate is mainly reduced to ethanol ${ }^{74}$. This is accomplished by the pyruvate decarboxylase that catalyzes the decarboxylation of pyruvate to acetaldehyde, and the alcohol dehydrogenase that reduces acetaldehyde to ethanol, resulting in two ATP equivalents ${ }^{74}$. Both reactions happen in the cytosol of the cells. In addition, the resulting acetaldehyde after decarboxylation of pyruvate can be further oxidized to acetate by the cytosolic aldehyde dehydrogenase and ultimately processed to acetyl-coenzyme A (acetyl-CoA) by the acetyl-CoA synthetase. Acetyl-CoA can then be transported to mitochondria for full respiration. Glucose induces repression of genes encoding proteins involved in the respiratory pathway, the so-called glucose or catabolite repression ${ }^{75-77}$.

In contrast to the fermentable carbon source glucose, the non-fermentable carbon source glycerol is metabolized differently and its metabolic flux is still under debate ${ }^{78}$. In S. cerevisiae, glycerol is mainly catabolized via the L-G3P pathway. Glycerol is phosphorylated to L-glycerol-3-phosphate (L-G3P) by the glycerol kinase and subsequently oxidized to dihydroxyacetone phosphate by the FAD-dependent glycerol-3phosphate dehydrogenase ${ }^{79}$. Dihydroxyacetone phosphate, in turn, is then channeled to the glycolysis pathway. The resulting pyruvate is transported into mitochondria and processed to acetyl-CoA by the pyruvate dehydrogenase complex (PDH). Acetyl-CoA enters the tricarboxylic acid (TCA) cycle in which it gets further oxidized to oxaloacetate in a series of ten reactions. These reactions create redox equivalents such as NADH and $\mathrm{FADH}_{2}$ that are, in turn, shuttled to the oxidative phosphorylation system to ultimately reduce molecular oxygen to water. The respiratory pathway results in 18 ATP equivalents in $S$. cerevisiae. Mitochondria also possess an aldehyde dehydrogenase (Ald4 $)^{80}$, analogous to that found in the cytosol, and uses it to by-pass the PDH, similar to the acetylCoA generation in the cytosol when glucose is used as carbon source. Acetaldehyde that is produced by the pyruvate decarboxylate can be oxidized to acetate by Ald4, while NAD+ is reduced to NADH in the matrix and can be directly channeled to the respiratory chain. Acetate is then processed to acetyl-CoA in the cytosol, which, in turn, is again channeled to the TCA cycle. In the absence of glucose, catabolite repression is abolished resulting in 
higher abundances of proteins that are involved in the TCA cycle and the OXPHOS system $^{72}$.

\subsection{The oxidative phosphorylation system}

The oxidative phosphorylation (OXPHOS) describes the synthesis of energy-rich ATP molecules by oxidation of the redox equivalents $\mathrm{NADH}$ and $\mathrm{FADH}_{2}$, the reduction of molecular oxygen to water and by phosphorylation of ADP to ATP. This is accomplished by chemiosmosis, hypothesized by Mitchell in $1961^{81}$. The catabolism of carbon sources such as glucose and glycerol to pyruvate, and their full oxidation via acetyl-CoA to oxaloacetate in the TCA cycle, transfer electrons to NAD+ and FAD. Shuttled by these redox equivalents, electrons are then transferred to complexes of the ETC, embedded in the IM of mitochondria. Complex I $(\mathrm{Cl})$ - known as the NADH:ubiquinone oxidoreductase in mammals - and complex II (CII) - the succinate:ubiquinone oxidoreductase - are the first contact points of $\mathrm{NADH}$ and $\mathrm{FADH}_{2}$ for electron transfer ${ }^{82}$. Ubiquinol shuttles the electrons from $\mathrm{Cl}$ and $\mathrm{CII}$ to complex III (CIII) - the ubiquinol:cytochrome coxidoreductase - which, in turn, transfers electrons to cytochrome $c$. Ultimately, cytochrome $c$ transfers electrons to complex IV (CIV) - the cytochrome coxidase - that finally reduces molecular oxygen to water. Redox reactions within the complexes are accompanied by electron acceptors and donors such as iron-sulfur clusters, heme groups and copper ions by which energy is transferred. $\mathrm{Cl}, \mathrm{CIII}$ and CIV use this energy to pump protons from the matrix into the IMS, creating an electrochemical gradient. Yeast, however, does not harbor $\mathrm{Cl}$ but internal and external NADH:ubiquinone oxidoreductases - Ndi1 located in the matrix and Nde1 and Nde2 in the IMS - that function as electron acceptors without pumping protons ${ }^{83}$. The electrochemical gradient is ultimately utilized by the $F_{1} F_{0}$ ATP synthase, often referred to as complex $\mathrm{V}(\mathrm{CV})$, also embedded in the IM. The created proton gradient is released by $\mathrm{CV}$ which uses the energy to phosphorylate ADP to ATP.

\subsubsection{Organization of the OXPHOS complexes}

Two models were initially proposed to describe the organization of the OXPHOS complexes: the "fluid state" and the "solid state" model ${ }^{84,85}$. Back in 1986, Hackenbrock et al. ${ }^{86}$ summarized experimental evidences supporting the "fluid state" model. This model describes a random distribution of all OXPHOS complexes within the IM of mitochondria. It claims that electron transfer is accomplished by freely diffusing ubiquinone and cytochrome $c$ pools randomly interacting with the complexes of the ETC, freely floating through the lipid bilayer as well. This, indeed, is supported by kinetic studies ${ }^{84}$. However, 
in the last two decades, the "solid state" model gained more and more attention. Highmolecular weight supercomplexes containing $\mathrm{CI}$, CIII and CIV in mammals and CIII and CIV in yeast were verified in different stoichiometries by blue native-polyacrylamide gel electrophoresis (BN-PAGE) after mild solubilization of mitochondria ${ }^{87,88} \cdot \mathrm{A} \mathrm{Cl}_{1} \mathrm{ClI}_{2} \mathrm{CIV}_{1}$ supercomplex is present in mammals and either a $\mathrm{ClI}_{2} \mathrm{CIV}_{2}$ or a $\mathrm{CIII}_{2} \mathrm{CIV}_{1}$ supercomplex in yeast. These supercomplexes were termed "respirasomes". Functional studies on respirasomes corroborated their existence ${ }^{89}$. Further evidence for supercomplexes were provided by medium-to-high-resolution structures obtained by cryogenic-electron microscopy (cryo-EM) ${ }^{90-93}$. The structure of the yeast $\mathrm{CIII}_{2} \mathrm{CIV}_{(2)}$ supercomplex could be solved with a resolution of $3.35 \AA^{91}$, and that of the human $\mathrm{Cl}_{1} \mathrm{CIII}_{2} \mathrm{CIV}_{1}$ with a resolution of $3.90 \AA^{90}$. In the latter study, even a $\mathrm{Cl}_{2} \mathrm{ClII}_{2} \mathrm{CIV}_{2}$ megacomplex with densities for Cll was proposed. By biochemical approaches such higher-order supercomplex structures were also shown to include the $F_{1} F_{0}$ ATP synthase ${ }^{94}$ ("respiratory string", human), the TIM23 complex $^{95,96}$ (yeast) or the ADP/ATP carrier protein ${ }^{97,98}$ (Pet9, yeast). In addition, chemical cross-linking in combination with mass spectrometry (XL-MS) found in organello evidence for supercomplex formation in murine ${ }^{99,100}$, human ${ }^{101}$ and yeast ${ }^{102}$ mitochondria by identifying subunits of $\mathrm{Cl}, \mathrm{CIII}$ and CIV that are in close proximity to each other (up to $\sim 4 \AA$ radius). Nonetheless, neither the "fluid state" nor the "solid state" model explains all experimental evidences properly. Therefore, Acín-Pérez and Enríquez ${ }^{103}$ proposed the "plasticity" model claiming that free and assembled complexes underlie a dynamic process that enables the cell to adapt to special growth conditions ${ }^{104}$.

\subsubsection{Biological significance for supercomplex formation}

Against the background of a "plasticity" model, the biological significance for supercomplex formation is still under debate. Three major functions have been proposed ${ }^{87}$ : substrate channeling, complex stabilization and sequestration of reaction intermediates. In a supercomplex arrangement of $\mathrm{Cl}, \mathrm{CIII}$ and CIV in mammals and of CIII and CIV in yeast, electron transport via ubiquinone and cytochrome $c$ are not dependent on random diffusion. The electron transporters can be channeled between the complexes that come along with catalytic enhancement. Indeed, no pool behavior of ubiquinone and cytochrome $c$ could be demonstrated in yeast ${ }^{105}$. In contrast, such pool behavior was reported in mammals ${ }^{106}$. Here, supercomplex formation contributes to the stabilization of $\mathrm{Cl}$. In the absence of $\mathrm{CIII}, \mathrm{Cl}$ is not stable ${ }^{107}$. $\mathrm{Cl}$ also functions as a scaffold for a supercomplex formation with $\mathrm{CIII}$ and $\mathrm{CIV}^{108}$. A tight interaction between these complexes might also be beneficial for reducing reactive oxygen species (ROS) such as superoxide ${ }^{109}$. ROS are involved in apoptosis ${ }^{110}$, amongst other processes. ROS are generated by leaky electron 
transfer of $\mathrm{Cl}$ and $\mathrm{CIII}$, hence, a close interaction of both complexes might prevent ROS generation ${ }^{87}$.

\subsubsection{Respiratory supercomplex factors}

After identifying ETC supercomplexes and debating their biological significance, researchers also focused on deciphering which components are needed for stabilization, assembly and regulation of supercomplexes. Especially when assuming a dynamic (de-)assembling as proposed by the "plasticity" model, factors must be present that aid the organization of supercomplex formation. One major player in supercomplex stability is the mitochondrial lipid cardiolipin. The group of Professor William Dowhan carved out that cardiolipin aids the formation and stability of a CIII-CIV supercomplex in yeast ${ }^{111-113}$, however, Pfeiffer et al. ${ }^{114}$ proved that cardiolipin is not essential for supercomplex formation. It could further be demonstrated that the ADP/ATP carrier protein strongly associates with the CIII-CIV supercomplex in yeast ${ }^{97,98}$ and that the absence of the carrier protein disturbs supercomplex formation and reduces respiratory activity. Recently, respiratory supercomplex factors (Rcf proteins 1-3) were described to interact with CIIICIV supercomplexes and to affect their formation. Rcf1 is a member of the conserved Hig1 (hypoxia-induced gene 1 ) type 2 family present in yeast and human cells ${ }^{115-117}$. It supports supercomplex formation and in its absence CIV's activity is significantly reduced and the formation of a CIII-CIV supercomplex is impaired ${ }^{115,116,118}$. Rcf2, also a member of the Hig1 type 2 family, and Rcf3, a yeast-specific protein, were shown to associate with the CIIICIV supercomplex ${ }^{115,116,119}$. A density as part of the yeast $\mathrm{CIII}_{2} \mathrm{CIV}_{(2)}$ supercomplex structure recorded by cryo-EM was recently identified as $\operatorname{Rcf} 2^{120}$. Rcf proteins play a role in supercomplex assembly and regulation, but the exact function still has to be interrogated.

\subsubsection{Assembly of the cytochrome $c$ oxidase}

The cytochrome $c$ oxidase, CIV, plays a special role in the respiratory chain. CIV is the terminal complex in the ETC that ultimately reduces molecular oxygen to water. CIV deficiencies are linked to diseases such as the Leigh syndrome, cardiomyopathy or Leber Hereditary Optic Neuropathy $(\mathrm{LHON})^{121}$. The complex consists of twelve subunits in yeast $^{120}$ and 13 subunits in humans ${ }^{122}$. The catalytic core subunits Cox1-3 are encoded by the mitochondrial DNA, while all other subunits (Cox4-9, Cox12, Cox13 and Cox26 in yeast) are encoded by the nuclear genome and have to be imported into mitochondria. These accessory subunits are required for complex assembly, stability and function ${ }^{123}$. A 
recently published near-atomic resolution structure of the yeast $\mathrm{CIII}_{2} \mathrm{CIV}_{2}$ supercomplex obtained by cryo-EM covered all subunits of CIV ${ }^{91}$, including peripheral subunits Cox12 and Cox13 and also the recently identified new subunit Cox26 $6^{124,125}$. The same group could also publish a comparable structure containing Rcf2 in yeast cells expressing the hypoxic isoform Cox $5 b^{120}$, but was not able to identify Rcf1 and Rcf3. The assembly of CIV is a well-coordinated process that involves more than 30 auxiliary factors ${ }^{123,126}$. Cox 1 is the first component that acts as a seed in the CIV assembly line (Figure 2). Cox1 subsequently forms an intermediate complex with Cox $5 \mathrm{a} / \mathrm{b}$ and Cox6 in yeast before assembling the catalytical core of CIV by the addition of Cox2 and Cox ${ }^{127,128}$. Next, preassembled Cox7Cox8-Cox9 are attached to the complex ${ }^{129}$, including Cox4. In a late stage of the assembly line, subunits Cox12 and Cox13 are added to the periphery of the complex, finalizing the mature $\mathrm{CIV}^{130}$.

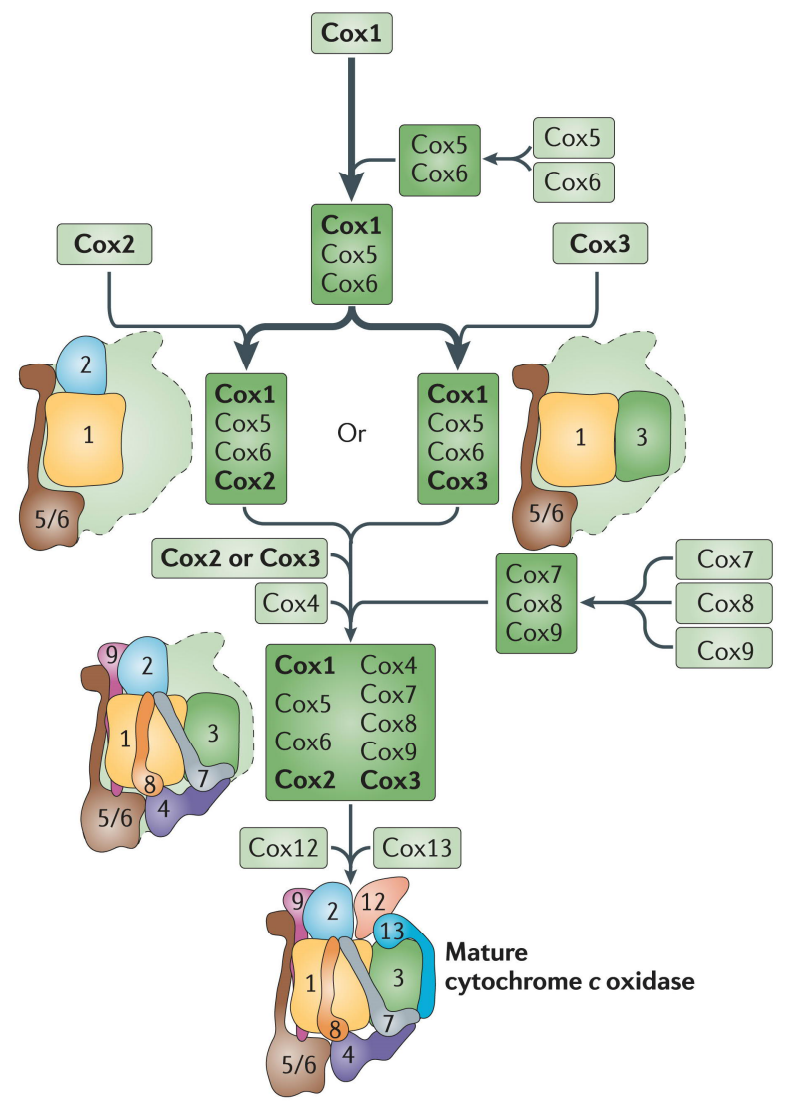

Figure 2: Cytochrome $c$ oxidase (complex IV, CIV) assembly in yeast mitochondria. Cox1 acts as seed and assembles with Cox5 and Cox6 prior to forming the catalytical core with Cox2 and Cox3. Cox7, Cox8 and Cox9 are preassembled before being attached to the complex together with Cox4. Cox12 and Cox13 are assembled in a late stage, ultimately forming the mature CIV. Translation and assembly factors are not included. Adapted by permission from Mick, Fox and Rehling ${ }^{122 .}$ 
However, Cox12 and Cox13 are not essential for the complex's function ${ }^{131,132}$. In recent times, an increasing number of assembly and translation factors have been identified that aid the assembly process. Most of the so far detected auxiliary factors are supposed to be involved in the early stage of CIV assembly ${ }^{133-136}$, in contrast to the Rcf proteins that are attached to the periphery of CIV and support supercomplex formation with CIII. In this context, Vukotic et al. ${ }^{115}$ could show that mature CIV displays heterogenous subunit compositions: the more abundant fraction represents CIV without Cox13, while only a minor fraction of CIV contains Cox13. Although being one of the most well-known complexes of the ETC supported by more than six decades of research, there are still new details about CIV to be unraveled. Especially the composition of intermediates, their interactions with auxiliary factors and their role in supercomplex formation need further evaluation.

\subsection{Mass spectrometry-based proteomics}

Proteins are biomolecules that execute most of the functions to keep a cell alive. They are involved in all biological processes, e.g. in metabolism, signaling cascades, DNA replication, transcription, translation, and the regulation of all these processes. The proteome describes the entity of proteins that are expressed in an organism under a specific condition at a specific time point ${ }^{137}$. The proteome can vary in protein composition, post-translational modifications of proteins and their abundances as a response to diseases, stress or environmental conditions. Additionally, composition and structural arrangement of protein complexes underlie those changes. The detection of changes of the proteome and its proteins is of utmost importance to investigate biological processes and how they are affected by diseases. Mass spectrometry (MS)-based proteomics is a powerful large-scale technique to analyze thousands of proteins in a sample ${ }^{138}$. The technique was successfully applied to interrogate the proteome of yeast mitochondria ${ }^{139-}$ ${ }^{141}$, localizations of mitochondrial proteins ${ }^{142-144}$ and changes in protein abundances in mitochondria upon a metabolic shift of $S$. cerevisiae from fermentative to respiratory conditions $^{72,145-148}$.

\subsubsection{General setup and principle of MS-based proteomics}

The duty of a mass spectrometer is to measure the mass-to-charge $(\mathrm{m} / \mathrm{z})$ ratios of analytes. In the field of proteomics, such analytes are peptides in the "bottom-up" approach (Figure 3), in contrast to the "top-down" approach where $\mathrm{m} / \mathrm{z}$ ratios of intact proteins are measured. Peptides are typically generated by digestion of the proteins within a sample 
by sequence-specific endoproteases such as trypsin ${ }^{149}$. To account for the complexity of a sample it can be fractionated on protein or peptide level prior to mass spectrometric analysis, e.g. based on size (size exclusion chromatography, gel electrophoresis) or charge (strong cation/anion exchange), amongst others ${ }^{150,151}$. To further reduce the number of peptides that enter the mass spectrometer at the same time, peptides are separated based on hydrophobicity under acidic conditions by reversed-phase liquid chromatography (LC) that is coupled to the mass spectrometer (LC-MS). During the elution process, protonated and, hence, positively charged peptides are ionized by a soft ionization method called electrospray ionization $(\mathrm{ESI})^{152}$. Due to an electric field that is applied between the spray needle and the mass spectrometer, small droplets carrying the peptides are formed that ultimately result in charged peptides in the gas phase after solvent evaporation in the ion source of the mass spectrometer. Besides the ion source, mass spectrometers generally consist of a mass analyzer and a mass detector. Contemporary instruments harbor a quadrupole as mass analyzer - or mass filter - and an orbitrap as additional analyzer and detector, such as $Q$ Exactive instruments by Thermo Fisher Scientific ${ }^{153,154}$. The peptide - or ion - beam is first focused by an ion optic system consisting of lenses in the vacuum. The beam then passes the quadrupole that is composed of four parallelly organized metal rods. By an interplay of direct and alternating voltages between the rods, ions with a specific $m / z$ ratio are forced to pass through the quadrupole on a stable trajectory while the other ions hit the metal rod and are thus sorted out $^{155}$. In a first full (also known as survey, precursor or MS1) scan, all ions are allowed to pass through the quadrupole. They are then analyzed in the orbitrap ${ }^{138}$. The orbitrap is composed of a barrel-shaped outer electrode and a spindle-shaped inner electrode. Entering ions harmonically oscillate around the inner electrode with a frequency proportional to $(\mathrm{m} / \mathrm{z})^{-1 / 2}$, generating image currents ${ }^{156}$. Image currents are finally Fouriertransformed into a mass spectrum indicating the abundance and the $m / z$ ratio of a specific ion species ${ }^{156}$. In the following MS2 scans, precursor ions are selected for fragmentation ${ }^{138}$. The commonly applied procedure for the selection of precursor ions is the data-dependent acquisition (DDA), also referred to as shotgun proteomics. The most intense precursor ions in an MS1 scan are allowed to pass through the quadrupole and are then fragmented in a collision cell. In a process called collision-induced dissociation (CID) or higher-energy collisional dissociation (HCD), ions are accelerated to collide with an inert gas such as nitrogen ${ }^{157,158}$. Peptides tend to break at the peptide bond, forming so-called $\mathrm{b}$ - and $\mathrm{y}$-fragment ions where the positive charge resides at the $\mathrm{N}$ - or the $\mathrm{C}$ terminus of the peptide, respectively ${ }^{159}$. Fragment ions are then guided to the orbitrap that detects their $\mathrm{m} / \mathrm{z}$ ratio. MS2 scans provide another level of information. Besides the analysis of the precursor ion that provide the mass of the peptide, the MS2 scans of the 
corresponding fragment ions provide sequence information of the peptide (LC-MS/MS). From the sequence of the peptide, the corresponding protein that was originally in the sample can be inferred ${ }^{138}$. Besides the data-dependent acquisition, mass spectrometers can be used to specifically search for peptides in a targeted approach (targeted proteomics $)^{160}$. For this, prior knowledge about the respective protein(s) of interest are required. In recent years, data-independent acquisition (DIA) has become increasingly popular. Precursor ions are no longer selected for fragmentation based on their intensity. Instead, all detected precursors in defined $\mathrm{m} / \mathrm{z}$ windows are subjected to MS2 fragmentation ${ }^{161,162}$.

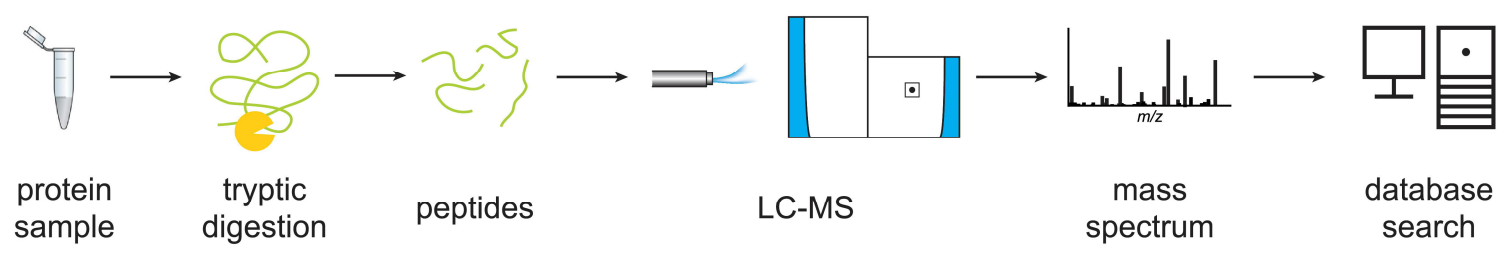

Figure 3: Classical shotgun proteomics workflow. A protein sample is tryptically digested to peptides and measured by liquid chromatography coupled to mass spectrometry (LC-MS). The resulting mass spectra are analyzed by database searching.

However, resulting MS2 spectra are chimeric and need to be analyzed with spectral libraries that have to be acquired in prior DDA measurements ${ }^{163}$. At this point, it should also be mentioned that different setups for mass spectrometers exist, mainly differing in the combination of mass analyzers and mass detectors. For example, time-of-flight (TOF) analyzers calculate masses of precursor ions via time that they need to pass a flight tube and reach the detector ${ }^{164}$. Modern tribrid instruments that harbor an ion trap as additional mass analyzer - in combination with a quadrupole and an orbitrap - are also capable of performing multi-tier MS scans $\left(\mathrm{MS}^{n}\right)^{165}$. Fragment ions can be further selected for another fragmentation and can then either be detected in the orbitrap or by secondary electron multipliers attached to the ion trap (MS3).

\subsubsection{Protein identification}

Peptide sequence identification and protein inference, i.e. the assignment of identified peptide sequences to the corresponding proteins, is performed by specific software with integrated search algorithms. The basic principle of peptide identification is based on the comparison of acquired spectra with in silico generated spectra after computational digestion of proteins within a dedicated database. The quality of a matched spectrum is 
expressed by a scoring model, e.g. based on a cross-correlation score applied by the Sequest algorithm ${ }^{166}$ or a probabilistic scoring model applied by the Mascot algorithm ${ }^{167}$. If a spectrum score is higher than a defined cut-off, the spectrum is considered as a peptide spectrum match (PSM). To statistically control matches that were wrongly assigned by the search algorithms amongst all PSMs, a false discovery rate (FDR) based on a targetdecoy approach is calculated ${ }^{168,169}$. The dedicated database not only contains true protein sequences - the targets - but also reversed or shuffled sequences of the respective proteins - the decoys. A misassigned PSM to a decoy protein is a false positive match. By applying an FDR cut-off, usually 1 to $5 \%$, it is defined how many false positive matches are tolerated amongst all PSMs. Identified peptide sequences that are unique in the database are then assigned to the respective proteins.

\subsubsection{Protein quantitation}

Not only the protein composition of a sample set is important to address biological questions but also changes of protein abundances between two different conditions of a system provide valuable information. Various quantitation methods have been introduced in the past, tailored to the power of mass spectrometry to analyze thousands of proteins in a sample. In general, two different approaches can be distinguished: the label-based and the label-free quantitation ${ }^{170}$. As part of the label-based quantitation, metabolic and chemical labeling have to be mentioned. The most prominent example for metabolic labeling is stable isotope labeling by amino acids in cell culture (SILAC) ${ }^{171}$. With this approach cells incorporate heavy labeled amino acids that are provided with the cell culture medium $-{ }^{13} \mathrm{C}-/{ }^{15} \mathrm{~N}$-labeled lysine and/or arginine amino acids - into newly synthesized proteins and can be compared with unlabeled cells. Nowadays, this method is also applicable to label mice ${ }^{172}$. SILAC can additionally be used to label auxotrophic (in regard of lysine and arginine synthesis $)^{173}$ and even prototrophic ${ }^{9}$ yeast strains. The introduced mass shift can be detected by LC-MS/MS, and peptides identified in both conditions can be relatively quantified. This is possible because the detected peak intensities are proportional to the analyte's concentration. The benefit of this approach is its resistance to random errors because the labels are introduced as early as possible in the workflow. Chemical labeling, in turn, is performed on either protein or peptide level after their synthesis in the organism ${ }^{170}$. One example for chemical labeling are tandem mass tags (TMT $)^{174}$. With this approach, labels are introduced on peptide level after enzymatic cleavage of the proteins. TMTs are isobaric and react with $\varepsilon$-amino groups of lysine residues or with protein and peptide $\mathrm{N}$-termini. They additionally support multiplexing, i.e. the parallel analysis of up to 16 conditions, an advantage when compared 
to SILAC labeling. Another difference to SILAC is the quantitation on MS2 level. Isobaric labeled peptides elute simultaneously from the analytical column and show identical behavior on MS1 level, resulting in one peak that is selected for fragmentation. During fragmentation, the tags will be cleaved and create unique non-isobaric reporter ions. By detecting these reporter ions and their intensity, accurate relative quantitation of peptides can be performed ${ }^{174}$. An increase of accuracy due to a decrease of precursor interferences can be achieved by an additional MS3 fragmentation of the isobaric peptides ${ }^{175}$. TMT labeling greatly reduces missing values across conditions ${ }^{176,177}$. Label-free quantitation is the least expensive method of all introduced so far, but also the method that suffers the most from random errors during the workflow. In DDA approaches, the label-free quantitation is performed by integrating peak intensities of peptide precursor ions on MS1 level that are then compared amongst different conditions. In DIA approaches, in turn, the quantitation is preferably carried out by integrating peak intensities of fragment ions of corresponding peptides on MS2 level. This was shown to be less susceptible to interferences from co-eluting peptides compared to MS1 level quantitation while additionally reducing coefficients of variation ${ }^{161,163}$.

\subsection{Cross-linking mass spectrometry}

Another tier of information to a biological question can be added by the detection of structural arrangements of proteins and protein-protein interactions (PPIs). Immunoprecipitations and affinity purifications are commonly used low-throughput methods for interrogating PPIs. Proximity labeling strategies like BiolD ${ }^{178}$ and APEX ${ }^{179}$ where proteins are genetically fused with an enzyme that adds a detectable modification to proteins in close proximity become increasingly popular. PPIs can also be detected by chemical cross-linking in combination with mass spectrometry $(X L-M S)^{180,181}$. Since proteins can be cross-linked to each other in solution forming protein-protein cross-links, $\mathrm{XL}-\mathrm{MS}$ is suitable to provide low resolution structural information of proteins, their interaction partners and their dynamics in a near-native environment. Results are, therefore, complementary to those obtained by traditional structure-solving techniques such as X-ray crystallography or cryo-EM that rely on rigid protein conformations ${ }^{182}$.

\subsubsection{Principle of XL-MS}

In a cross-linking reaction a chemical ingredient reacts with functional groups of amino acids of proteins that are in close proximity to each other ${ }^{180}$. Commonly used homobifunctional cross-linkers such as disuccinimidyl suberate (DSS) and its derivative 
bis(sulfosuccinimidyl) suberate (BS3) harbor two $\mathrm{N}$-hydroxysuccinimide (NHS) esters separated by six methylene groups, the spacer ${ }^{183}$. Both NHS esters are targets of nucleophiles to whom $\varepsilon$-amino groups of lysine residues and protein $\mathrm{N}$-termini with their free electron pairs of the nitrogen atoms can be considered as one of the strongest in a proteinaceous environment (Figure 4). Lysine residues and protein $\mathrm{N}$-termini are, hence, the most probable reactants to react with the NHS esters in a nucleophilic attack. Side reactions have been reported for the reaction of $\mathrm{NHS}$ esters with the $\mathrm{OH}$ groups of serine, threonine and tyrosine residues ${ }^{184-186}$. After reaction, the participating amino acids are covalently linked to each other, and separated by the spacer forming a residue-to-residue cross-link. Depending on the length of the spacer a specific distance constraint is introduced to the protein system. In case of BS3 and DSS the spacer spans a distance of $11.4 \AA$, which is usually expanded to $30 \AA$ considering the distance between Ca-atoms and accounting for flexibility ${ }^{187}$. Different cross-linkers harboring different spacer length and featuring different reaction chemistries are commercially available ${ }^{188}$. 1-ethyl-3-(3dimethylaminopropyl)carbodiimide hydrochloride (EDC), for example, cross-links lysine residues to amino acids containing carboxy groups, such as aspartate and glutamate, without a spacer in between, a zero-length cross-linker ${ }^{189}$. EDC contains two different reactive groups and is therefore a heterobifunctional cross-linker. A carbodiimide group first reacts with a carboxy group. By adding sulfo-NHS, EDC can then be stabilized by the formation of an NHS ester that, in turn, reacts with an amino group. In addition, crosslinkers with UV-inducible diazirines can react with essentially all amino acids in a radical reaction ${ }^{190}$, or maleimide-containing cross-linkers connect sulfhydryl-groups of cysteine residues with each other ${ }^{191}$. The covalent bond induced by the cross-linker between two amino acid residues remains stable through the whole sample preparation workflow including denaturing, prefractionation, digestion, and enrichment, finally resulting in crosslinked peptides ${ }^{180}$. Consequently, distance information is conserved and can be detected by mass spectrometry revealing the exact amino acid residues that were involved in the cross-linking reaction. Cross-links can be categorized as mono-, intraprotein and interprotein cross-links ${ }^{192}$. Mono-cross-links originate from a cross-linking reaction where only one functional group of the cross-linker reacted with an amino acid residue while the other one got hydrolyzed. These cross-links can provide information about solvent accessibility of amino acid residues on the surface of proteins. Intraprotein cross-links occur between amino acid residues of one protein and can give insights into its conformation or conformational changes of specific regions upon stimulation. The most informative cross-links, however, are interprotein cross-links, i.e. connections between two different proteins. From these cross-links, spatial arrangements of proteins in protein complexes and protein-protein interactions can be determined. 


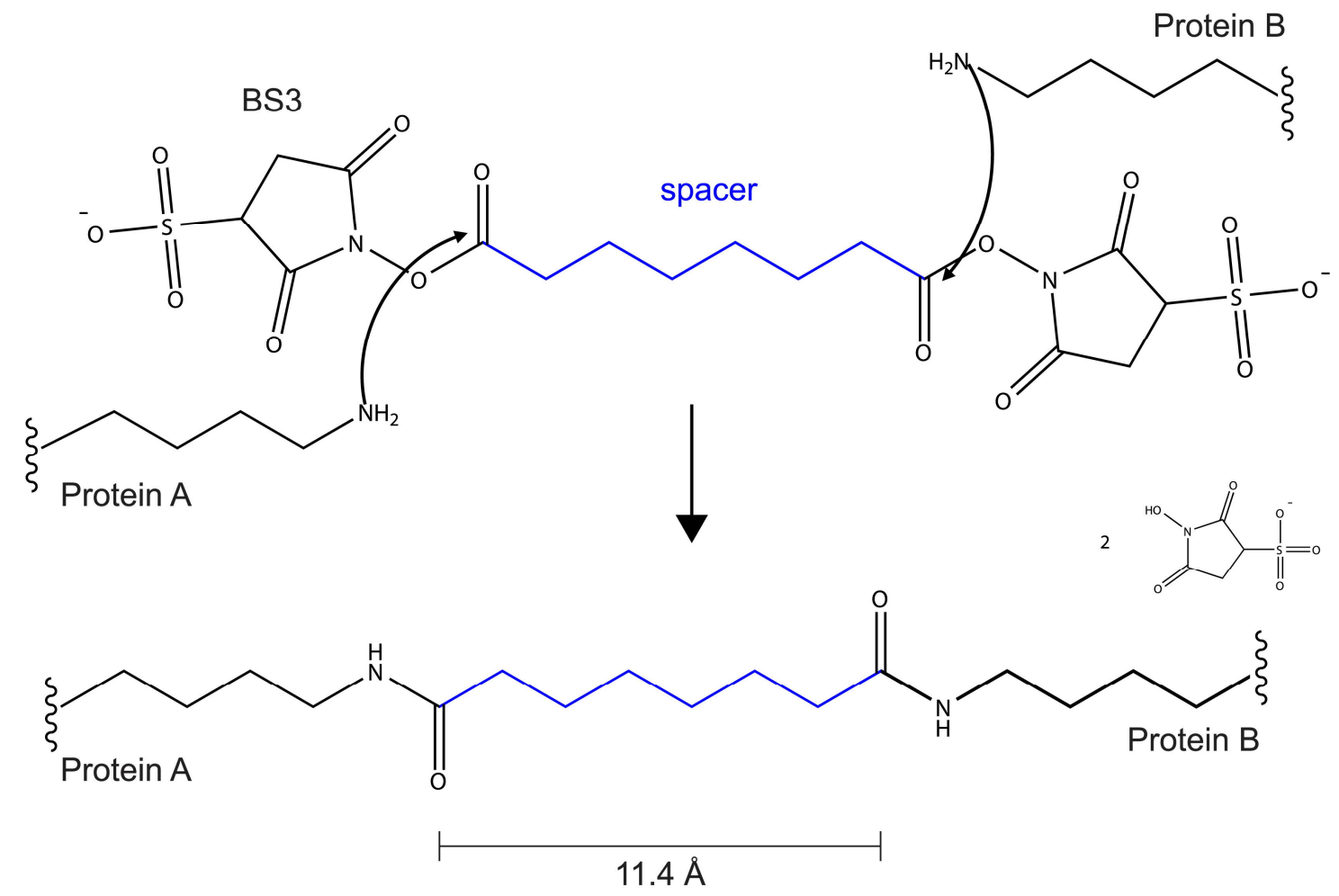

Figure 4: Reaction schema of protein-protein cross-linking with BS3. Lysine residues of Protein A and Protein $B$ undergo a nucleophilic attack with the free electron pair of the nitrogen atom of the $\varepsilon$-amino group on the carbonyl atom of the cross-linker. After successful reaction, Protein $A$ and Protein B are covalently linked to each other, separated by the spacer (11.4 $\AA$ in case of BS3). $N$ hydroxysulfosuccinimide (sulfo-NHS) is the leaving group.

\subsubsection{Analytical benefits of $X L-M S$}

In the early 2000s XL-MS began its rise in the analysis of the spatial organization of single proteins or protein complexes. As one of the first users, Young et al. ${ }^{193}$ could determine the fold of the growth factor FGF-2. In the same year, Rappsilber et al. ${ }^{194}$ analyzed the Nup85 complex by separating cross-linked from non-cross-linked complexes by gel electrophoresis prior to LC-MS/MS analysis. The analysis of larger protein complexes followed promptly, e.g. by Maiolica et al. ${ }^{195}$ who interrogated the structure of the human NDC80 heterotetramer or by Chen et al. ${ }^{196}$ revealing the architecture of the human RNA polymerase II-TFIIF complex. Especially the combination of XL-MS and cryo-EM experiments proved to be fruitful in structural biology ${ }^{182}$. Here, XL-MS can aid the positioning of protein subunits into cryo-EM densities where a low local resolution hampers the unambiguous identification of a protein. With this approach, subunits of the human spliceosome could be arranged correctly in its structure ${ }^{197,198}$. Also, the RNA polymerase II-mediator core initiation complex ${ }^{199}$ and the inner ring scaffold of the nuclear pore complex ${ }^{200}$ could be solved by cryo-EM in combination with XL-MS, amongst others. 
Distance constraints derived by XL-MS can also be used in integrative modeling approaches gathering scientific data of different structure biology-related fields to propose structures of macromolecular assemblies ${ }^{201}$ or by docking and modeling software such as HADDOCK $^{202}$ and Rosetta ${ }^{203}$. In recent years, XL-MS was increasingly applied to elucidate even more complex protein networks. For example, Herzog et al. ${ }^{204}$ identified the interaction network of human protein phosphatase $2 \mathrm{~A}$ after affinity-purification, crosslinking and LC-MS/MS analysis. Cross-linking of whole organisms ${ }^{205-207}$, cells ${ }^{208,209}$, organelles $^{99,100}$ and tissues ${ }^{210}$ to elucidate protein interactomes by XL-MS demonstrate the rapid development of this technique.

\subsubsection{Adapting the proteomics workflow for XL-MS}

XL-MS poses several challenges. While most steps of the standard proteomics workflow (Figure 3) can be copied, some steps have to be adapted for the successful analysis of cross-links. First, the optimal concentration of cross-linker has to be determined. If the concentration is too low, especially cross-links between low abundant proteins might not be detectable ${ }^{211}$. If the concentration is too high, the cross-linker can induce local structural disturbances within proteins ${ }^{212}$ and lead to artificial results. Especially when using NHS ester-based cross-linkers that are targeted by lysine residues, tryptic digestion will be hampered, since residues modified by the cross-linker will no longer be recognized as a substrate of trypsin. This has to be considered in the search parameters, since the number of missed cleavage sites for cross-linked peptides are higher than for linear, i.e. non-crosslinked peptides. Furthermore, the cross-linking efficiency is calculated to be $1-5 \%{ }^{213}$, hence, the vast majority of peptides after digestion represents linear peptides. To increase the identification rate, several strategies have been developed to enrich cross-linked peptides prior to LC-MS/MS analysis. Such strategies are either based on size via size exclusion chromatography (SEC) $)^{214}$, since cross-linked peptides are longer and bulkier than linear peptides, or based on charge via strong cation exchange $(S C X)^{215}$ chromatography, since cross-linked peptides are supposed to carry more positively charged amino acids. Another strategy to enrich for cross-linked peptides is the application of trifunctional cross-linkers. These cross-linkers harbor an additional enrichment group, such as biotin ${ }^{216-218}$ or phosphor-based tags ${ }^{213,219}$ that can be used to separate cross-linked from linear peptides. However, the biggest challenge is the data analysis. Spectra of crosslinked peptides are chimeric displaying fragment ions of both peptides and fragment ions that are shifted due to the introduced mass of the cross-linker. Database searches for the identification of cross-linked peptides have to be performed by different algorithms in contrast to the identification of linear peptides ${ }^{220}$. To identify a cross-linked peptide pair, 
proteins within a database will also be in silico digested in a similar way to the approach for linear peptides. However, the consideration of all peptide pair combinations sums up to $\left(n^{2}+n\right) / 2$ possibilities where $n$ is the number of peptides in the database, the so-called $n^{2}$ problem ${ }^{180,220}$. While this is still feasible for small databases after cross-linking single proteins or protein complexes, the identification of cross-linked peptides in more complex samples is tremendously challenging. The analysis of 50 cross-linked proteins creates a search space as large as the one for the analysis of linear peptides of the whole human proteome $^{220}$. This search space inflates quadratically with the number of proteins in the database for a cross-linked peptides search increasing the likelihood of identifying false positives. Additionally, three times more decoys are included in FDR calculation, since the combinations target-target, target-decoy, decoy-target and decoy-decoy have to be considered $^{220}$.

\subsubsection{Cross-linking of complex samples}

A popular strategy to overcome the $n^{2}$ problem is the application of MS-cleavable crosslinkers. These cross-linkers contain a labile bond that breaks in the gas phase during MS2 fragmentation in the mass spectrometer ${ }^{221}$. Due to the fragmentation of the labile bond within the cross-linker, both peptides that reacted with the cross-linker can be selected separately for another MS3 fragmentation event. In combination with resulting reporter ions due to the unequal cleavage of the labile bond peptides can be sequenced individually. In a subsequent search, the search space is then reduced to $2 n$, allowing the possibility to search against whole proteomes. Commonly used MS-cleavable crosslinkers are disuccinimidyl sulfoxide (DSSO) ${ }^{221}$, harboring a labile sulfoxide group and disuccinimidyl dibutyric urea $(\mathrm{DSBU})^{222}$, harboring a labile urea group. Both are homobifunctional NHS esters and, thus, primarily react with lysine residues and protein Ntermini, like BS3 and DSS. Another example is the protein interaction reporter (PIR) ${ }^{223}$ developed by the laboratory of Professor James Bruce that is also an NHS ester-based, cleavable, and also enrichable cross-linker. It additionally produces reporter ions upon fragmentation that indicate cross-linked peptides. By using MS-cleavable cross-linkers, studies on Drosophila melanogaster ${ }^{205}$, Escherichia coli ${ }^{206}$, HeLa ${ }^{209}$ and HEK ${ }^{217}$ cells provided promising insights into the interactome of complex systems. MS-cleavable crosslinkers were also used to cross-link isolated murine ${ }^{99,100}$ and yeast ${ }^{102}$ mitochondria. However, it is more likely that abundant proteins react with the cross-linker and whose peptides are finally identified as being cross-linked ${ }^{100,224}$. Ryl et al. ${ }^{101}$ could demonstrate on human mitochondria that the non-cleavable cross-linker DSS and a database search 
limited to the $\sim 1000$ most abundant proteins can also provide reliable protein-protein interactions in a crowded environment.

\subsubsection{Quantitative XL-MS}

To ultimately combine the power of mass spectrometry regarding the identification of protein-protein interactions, that is, chemical cross-linking, and the quantitation of protein abundance changes in samples originating from various conditions, quantitative crosslinking mass spectrometry ( $q X L-M S$ ) becomes increasingly popular. This enables the quantitation of both the changes of PPI networks between two conditions and the quantitative assessment of dynamic conformational changes within proteins or protein complexes upon stimulation. Especially for the latter one, qXL-MS can be a valuable tool, since traditional methods for structure elucidation such as X-ray crystallography or cryoEM depend on rigid and fixated protein complexes ${ }^{225}$. Quantitation approaches that were introduced for MS-based proteomics in subsection 1.7.3 can also be applied in qXL-MS. In addition, isotopically labeled cross-linkers can be utilized to connect proteins and protein networks under different conditions ${ }^{226}$. For example, a 4 Da mass shift can be introduced by BS3-d4 to proteins originating from condition $A$, while proteins originating from condition $B$ are cross-linked with unlabeled BS3. This shift can be used to relatively quantify crosslinked peptide pairs, like the SILAC approach. By cross-linking native and dephosphorylated $F_{1} F_{0}$ ATP synthase of chloroplasts, Schmidt et al. ${ }^{227}$ could demonstrate the feasibility of introducing mass shifts by labeled cross-linkers for the quantitation of protein complexes in two different conditions. This approach is now widely used to probe conformational changes within proteins ${ }^{228-232}$. An approach for quantitation of cross-links on MS2 level was introduced by $\mathrm{Yu}$ and colleagues ${ }^{233}$. They cross-linked the electron carrier cytochrome $c$ with the MS-cleavable cross-linker DSSO and labeled the peptides with TMT. Also, label-free approaches were tested for cross-linked proteins with quantitation on MS1 level by $\operatorname{DDA}^{234}$ and on MS2 level by DIA ${ }^{235}$. However, all these approaches need further evaluation on more complex samples. Chavez et al. ${ }^{208}$, in turn, combined XL-MS with SILAC labeling to compare treated and untreated multidrugresistant human carcinoma cells. This approach is especially beneficial for complex samples since it provides not only information about conformational changes or changes within the PPI network, but also about changes in protein abundances. Although qXL-MS for large-scale experiments is still challenging, mainly due to the low signal intensities of cross-linked peptides resulting in missing values amongst conditions, it can already provide valuable information on changes of PPI networks and protein conformations ${ }^{236}$. 


\subsection{Scope of the thesis}

The energy production in Saccharomyces cerevisiae depends on the available carbon source. Under fermentative conditions, ATP is produced primarily by fermentation. Under non-fermentable conditions, the carbon source is fully oxidized via the tricarboxylic acid (TCA) cycle and the oxidative phosphorylation (OXPHOS) system located within mitochondria. Yeast cells regulate involved pathways by catabolite repression of genes encoding proteins participating e.g. in the OXPHOS system. This gene regulation affects abundances of mitochondrial proteins, their interaction with each other, and, consequently, the ultrastructure of mitochondria in different growth conditions. Protein-protein crosslinking in combination with mass spectrometry (XL-MS) proved to be a powerful tool to identify large-scale protein-protein interactions (PPIs) and their dynamical changes. Several challenges were addressed within the scope of this thesis: (i) Protein-protein cross-linking networks within mitochondria derived from yeast grown on either glucosecontaining, a fermentable carbon source, or on glycerol-containing medium, a nonfermentable carbon source, were interrogated by XL-MS and compared to each other qualitatively. In general, so far undescribed PPIs were detected with the focus on supercomplex formation in the electron transport chain. (ii) Changes of protein-protein cross-links within mitochondria of both conditions were also tracked by a quantitative XLMS approach. (iii) To address interactions between low abundant proteins, protein-protein cross-links within mitochondria were also elucidated in a $r \mathrm{o}^{\circ}$ yeast strain lacking mitochondrial DNA. (iv) Cross-linkers with different reaction chemistries were used to investigate protein-protein cross-linking networks in mitoplasts derived from human mitochondria. In summary, this thesis provides qualitative and quantitative information about protein-protein cross-linking networks within mitochondria derived from yeast grown under different conditions as well as human mitoplasts acquired by XL-MS. 


\section{Materials and Methods}

\subsection{Materials}

\subsubsection{Chemicals and buffers}

If not otherwise stated, commonly used chemicals and ingredients were purchased from Merck Millipore (Billerica, USA), Sigma-Aldrich (St. Louis, USA) or Thermo Fisher Scientific (Waltham, USA).

Table 1: List of used chemicals and suppliers.

\begin{tabular}{|c|c|}
\hline Chemical & Supplier \\
\hline [35S]-L-Methionine & Hartmann Analytic \\
\hline $\begin{array}{l}\text { 1-ethyl-3-(3-dimethylaminopropyl)carbodiimide } \\
\text { hydrochloride (EDC) }\end{array}$ & Thermo Fisher Scientific \\
\hline Acetonitrile, LiChrosolv & Merck Millipore \\
\hline Ammonium bicarbonate (ABC) & Sigma-Aldrich \\
\hline Antimycin A & Sigma-Aldrich \\
\hline Adenosine diphosphate (ADP) & Sigma-Aldrich \\
\hline Adenosine triphosphate (ATP) & Roche \\
\hline $\begin{array}{l}\text { Bis(sulfosuccinimidyl)suberate (BS3, No-Weigh } \\
\text { Format) }\end{array}$ & Thermo Fisher Scientific \\
\hline BS3-d4 (isotopically labeled, No-Weigh Format) & Thermo Fisher Scientific \\
\hline Bovine serum albumin (BSA) & Sigma-Aldrich \\
\hline $\begin{array}{l}\text { Carbonyl cyanide-4- } \\
\text { (trifluoromethoxy)phenylhydrazone (FCCP) }\end{array}$ & Sigma-Aldrich \\
\hline Coomassie InstantBlue & Merck Millipore \\
\hline $\mathrm{CuSO}_{4} \times 5 \mathrm{H}_{2} \mathrm{O}$ & Merck Millipore \\
\hline Cytochrome $c$ & Sigma-Aldrich \\
\hline Digitonin & Merck Millipore \\
\hline Dimethyl sulfoxide (DMSO) & Sigma-Aldrich \\
\hline Disuccinimidyl suberate (DSS, No-Weigh Format) & Thermo Fisher Scientific \\
\hline Dithiothreitol (DTT) & Merck Millipore \\
\hline Ethidium bromide $0.07 \%$ & AppliChem \\
\hline Formic acid (FA) & Sigma-Aldrich \\
\hline lodoacetamide (IAA) & Sigma-Aldrich \\
\hline Methanol, LiChrosolv & Merck Millipore \\
\hline n-Dodecyl $\beta$-D-maltoside (DDM) & Sigma Aldrich \\
\hline Nicotinamide adenine dinucleotide (NADH) & Roche \\
\hline N-hydroxysulfosuccinimide (sulfo-NHS) & Thermo Fisher Scientific \\
\hline$N$-ethylmaleimide (NEM) & Sigma-Aldrich \\
\hline Oligomycin & Sigma Aldrich \\
\hline Protein-A sepharose & GE Healthcare \\
\hline Proteinase $\mathrm{K}$, recombinant & Roche \\
\hline Sodium dodecyl sulfate (SDS) & Sigma-Aldrich \\
\hline
\end{tabular}




\begin{tabular}{ll}
\hline Sucrose & Roth \\
Trehalose & Sigma-Aldrich \\
\hline Trifluoroacetic (TFA) & Carl-Roth \\
\hline Trypsin & Promega, Sigma-Aldrich \\
Urea & Sigma-Aldrich \\
Water, LiChrosolv & Merck Millipore \\
Valinomycin & Merck Millipore \\
Zymolyase-20T & Seikagaku Biobusiness \\
& Corporation (Tokyo, Japan) and \\
& Nacalai Tesque Inc. (Kyoto, \\
& Japan) \\
\hline
\end{tabular}

Table 2: List of used buffers and their compositions.

\begin{tabular}{|c|c|}
\hline Buffer & Composition \\
\hline BN-PAGE loading dye & $\begin{array}{l}5 \% \text { Coomassie G-250, } 500 \text { mM 6-amino-hexanoic } \\
\text { acid and } 0.1 \text { M Bis-Tris pH } 7.0\end{array}$ \\
\hline $\begin{array}{l}\text { Cell culture medium HEK293T } \\
\text { cells }\end{array}$ & $\begin{array}{l}\text { Dulbecco's modified Eagle's medium (DMEM), } \\
10 \% \text { fetal bovine serum (FBS), } 2 \mathrm{mM} \mathrm{L-glutamine,} \\
1 \mathrm{mM} \text { Sodium pyruvate, } 50 \mu \mathrm{g} / \mathrm{mL} \text { uridine, } \\
100 \text { units/mL Penicillin, } 100 \mu \mathrm{g} / \mathrm{mL} \text { Streptomycin }\end{array}$ \\
\hline Cross-linking buffer & 20 mM HEPES pH 7.5, $100 \mathrm{mM} \mathrm{NaCl}$ \\
\hline DTT buffer & $100 \mathrm{mM}$ Tris $/ \mathrm{H}_{2} \mathrm{SO}_{4} \mathrm{pH} 9.4,10 \mathrm{mM}$ DTT \\
\hline EM buffer & 1 mM EDTA, 10 mM MOPS-KOH pH 7.2 \\
\hline $\begin{array}{l}\text { Homogenization buffer human } \\
\text { mitoplasts }\end{array}$ & $\begin{array}{l}300 \mathrm{mM} \text { trehalose, } 10 \mathrm{mM} \mathrm{KCl}, 10 \mathrm{mM} \text { HEPES } \\
\mathrm{pH} 7.4\end{array}$ \\
\hline $\begin{array}{l}\text { Homogenization buffer yeast } \\
\text { mitochondria }\end{array}$ & $\begin{array}{l}10 \mathrm{mM} \text { Tris pH 7.4, } 0.6 \mathrm{M} \text { sorbitol, } 125 \mathrm{mM} \text { EDTA } \\
\mathrm{pH} 8,2 \mathrm{~g} / \mathrm{L} \mathrm{BSA}, 50 \mathrm{mM} \text { PMSF }\end{array}$ \\
\hline Import buffer & $\begin{array}{l}250 \mathrm{mM} \text { sucrose, } 10 \mathrm{mM} \mathrm{MOPS} / \mathrm{KOH} \mathrm{pH} 7.2 \text {, } \\
80 \mathrm{mM} \mathrm{KCl}, 2 \mathrm{mM} \mathrm{K \textrm {K } _ { 2 } \mathrm { PO } _ { 4 } , 5 \mathrm { mM } \mathrm { MgCl }}, 5 \mathrm{mM} \\
\text { methionine, } 3 \% \text { fatty acid-free BSA }\end{array}$ \\
\hline Lysis buffer human mitoplasts & $\begin{array}{l}50 \mathrm{mM} \text { Tris pH 7.4, } 100 \mathrm{mM} \mathrm{NH}_{4} \mathrm{Cl}, 10 \mathrm{mM} \mathrm{MgCl}_{2} \text {, } \\
2 \% \text { SDS and in case of EDC cross-linking } 20 \mathrm{mM} \\
\text { DTT }\end{array}$ \\
\hline NuPAGE LDS sample buffer & $\begin{array}{l}106 \mathrm{mM} \text { Tris, } 141 \mathrm{mM} \text { Tris-Base, } 2 \% \text { LDS, } 10 \% \\
\text { glycerol, } 0.51 \mathrm{mM} \text { EDTA, } 0.22 \mathrm{mM} \text { SERVA Blue } \\
\text { G250, } 0.175 \mathrm{mM} \text { Phenol Red, } 50 \mathrm{mM} \text { DTT }\end{array}$ \\
\hline Respiration buffer & $\begin{array}{l}225 \mathrm{mM} \text { sucrose, } 75 \mathrm{mM}^{2} \text { mannitol, } 10 \mathrm{mM} \text { Tris } \\
\mathrm{pH} 7.4,10 \mathrm{mM} \mathrm{KH}_{2} \mathrm{PO}_{4}, 5 \mathrm{mM} \mathrm{MgCl}_{2}, 10 \mathrm{mM} \mathrm{KCl}\end{array}$ \\
\hline SEM/PMSF buffer & $\begin{array}{l}250 \mathrm{mM} \text { sucrose, } 20 \mathrm{mM} \text { MOPS pH 7.2, } 1 \mathrm{mM} \\
\text { EDTA, } 1 \mathrm{mM} \text { PMSF }\end{array}$ \\
\hline SH buffer & 0.6 M sorbitol, 20 mM HEPES pH 7.5 \\
\hline $\begin{array}{l}\text { Solubilization buffer yeast } \\
\text { mitochondria }\end{array}$ & $\begin{array}{l}20 \mathrm{mM} \text { Tris pH 7.4, } 50 \mathrm{mM} \mathrm{NaCl}, 10 \% \text { glycerol, } \\
5 \mathrm{mM} \text { EDTA, } 1 \mathrm{mM} \text { PMSF, } 1 \% \text { digitonin or } 0.6 \% \\
\text { DDM }\end{array}$ \\
\hline Protein SEC buffer & $20 \mathrm{mM}$ HEPES $\mathrm{pH} 8,100 \mathrm{mM} \mathrm{NaCl}$ and $0.1 \%$ SDS \\
\hline TBS-T & $20 \mathrm{mM}$ Tris pH 7.5, 62 mM NaCl, 0.1 \% Tween 20 \\
\hline TG buffer & 35 mM Tris, 220 mM glycine, $\mathrm{pH} 8.3$ \\
\hline Zymolyase buffer & $20 \mathrm{mM} \mathrm{K}_{2} \mathrm{HPO}_{4} / \mathrm{KH}_{2} \mathrm{PO}_{4} \mathrm{pH}$ 7.4, $1.2 \mathrm{M}$ sorbitol \\
\hline
\end{tabular}




\subsubsection{Equipment and consumables}

Table 3: List of commonly used consumables and suppliers.

\begin{tabular}{ll} 
Consumables & Supplier \\
\hline ANTI-FLAG M2 affinity gel & Sigma-Aldrich \\
HA-antibody columns & Sigma-Aldrich \\
\hline Immobilon ${ }^{\circledR}$-P PVDF membrane & Merck Millipore \\
NuPAGE 4-12 \% Bis-Tris gels & Invitrogen \\
\hline Pierce BCA Protein Assay & Thermo Fisher Scientific \\
Reprosil-Pur 120 C18-AQ, $1.9 \mu \mathrm{m}$ & Dr. Maisch GmbH \\
Roti $^{\circledR}$-Quant & Roth \\
SepPak C18 cartridges, $50 \mathrm{cc}$ & Waters \\
\hline
\end{tabular}

Table 4: List of regularly used equipment and suppliers.

\begin{tabular}{ll}
\hline Equipment & Supplier \\
\hline Agilent 1100 HPLC series & Agilent \\
ÄKTAmicro & GE Healthcare \\
\hline Avanti J-26 XP (centrifuge) & Beckman Coulter \\
Bioruptor sonication apparatus UCW-201TM & Diagenode \\
\hline Cooling microcentrifuge Heraeus Fresco17 & Thermo Fisher Scientific \\
Gel chamber Novex Mini-cell & Invitrogen \\
\hline Homogenplus Homogenizer & Schuett-Biotech \\
JA-20 (rotor) & Beckman Coulter \\
Lab scale BP 211D & Sartorius \\
Lab scale CPA 423S & Sartorius \\
\hline Mass Spectrometer Orbitrap Fusion Tribrid & Thermo Fisher Scientific \\
Mass Spectrometer Orbitrap Fusion Lumos & Thermo Fisher Scientific \\
Tribrid & \\
\hline Mass Spectrometer Q Exactive HF-X & Thermo Fisher Scientific \\
Microcentrifuge Heraeus Pico17 & Thermo Fisher Scientific \\
Nano-LC Dionex UltiMate 3000 RSLCnano & Thermo Fisher Scientific \\
Oxygraph 2k & Oroboros \\
Potter S (dounce homogenizer) & Sartorius \\
\hline Power supply power-pac 200 & Bio-Rad \\
\hline Precision scale BP 4100 & Sartorius \\
SMART system & Pharmacia Biotech \\
Sonication bath Sonorex & Bandelin \\
Sorvall H-12000 (rotor) & Thermo Fisher Scientific \\
Sorvall RC 12BP (centrifuge) & Thermo Fisher Scientific \\
Speedvac concentrator 5301 & Eppendorf \\
SuperdexPeptide 3.2/300 SEC column & GE Healthcare and Pharmacia \\
& Biotech \\
\hline Superose-6 Increase 10/300 SEC column & GE Healthcare \\
\hline Thermomixer C & Eppendorf \\
\hline & \\
\hline
\end{tabular}




\subsubsection{Software}

Table 5: List of used software with indicated version and developers.

\begin{tabular}{ll}
\hline Software & Developer \\
\hline Cytoscape 3.7.1 & Cytoscape Consortium \\
DisVis & Bonvin group, Utrecht University \\
\hline Mascot 2.3.02 & Matrix Science, Boston, USA \\
\hline MaxQuant 1.6.0.1 & Cox group, MPI of Biochemistry, Martinsried \\
\hline Perseus 1.6.0.7 & Cox group, MPI of Biochemistry, Martinsried \\
pLink 1.23 & pFind group, Institute of Computing Technology, Beijing \\
\hline pLink 2.3 & pFind group, Institute of Computing Technology, Beijing \\
\hline Proteome Discoverer 2.1 & Thermo Fisher Scientific \\
\hline PyMOL 2.3.3 & Schroedinger, LLC \\
\hline R 3.5.0 & R core team \\
\hline Rstudio 1.1.477 & Rstudio \\
\hline Scaffold 4 & Proteome Software, Inc., USA \\
Tune 2.9 & Thermo Fisher Scientific \\
UCSF Chimera 1.11.2/1.14 & RBVI, University of California \\
Xcalibur 4.1 & Thermo Fisher Scientific \\
Xlink Analyzer 1.1.4 & Kosinski group, EMBL Hamburg \\
xiNET & Rappsilber group, TU Berlin \\
xVis & Herzog group, LMU Munich \\
\hline
\end{tabular}

\subsection{Microbiological and biochemical methods}

\subsubsection{Saccharomyces cerevisiae growth conditions}

Saccharomyces cerevisiae originated from the strain YPH499 and was grown on medium containing $1 \%$ yeast extract, $2 \%$ peptone, and either $3 \%$ glycerol, $2 \%$ glucose or $3 \%$ lactate. The min8D strain was generated via homologous recombination of a HIS3MX6 cassette, and the Min8-HA as well as the Min8 ${ }^{\text {FLAG }}$ strain by chromosomal integration ${ }^{237}$.

\subsubsection{Generation of yeast $r h o^{0}$ strains}

Yeast cells lacking mitochondrial DNA ( $r h o^{\circ}$ strains) were generated by adding $25 \mu \mathrm{g} / \mathrm{mL}$ ethidium bromide to a culture grown on medium containing $1 \%$ yeast extract, $2 \%$ peptone, and $2 \%$ glucose for three days (modified from ref ${ }^{238}$ ). 


\subsubsection{Isolation of mitochondria from S. cerevisiae}

Mitochondria were isolated from yeast cells grown at $30^{\circ} \mathrm{C}$ as previously described ${ }^{239}$. Cells were harvested at $\mathrm{OD}_{600} 1.5-3$ in $2 \mathrm{~L}$ centrifuge beakers at 4,000 rpm (Sorvall $\mathrm{H}$ $12000 /$ Sorvall RC $12 \mathrm{BP}$ ) for $15 \mathrm{~min}$ at $18^{\circ} \mathrm{C}$. The supernatant was discarded, and the pellet was washed with $300 \mathrm{~mL}$ deionized water, transferred to $500 \mathrm{~mL}$ centrifuge beakers and spun down at $4,000 \mathrm{rpm}$ for $15 \mathrm{~min}$ at $18^{\circ} \mathrm{C}$. The supernatant was discarded and the cell wet weight (cww) was determined. Pellets were resuspended in dithiothreitol (DTT) buffer (2 mL/g cww, $100 \mathrm{mM}$ Tris/ $\mathrm{H}_{2} \mathrm{SO}_{4} \mathrm{pH}$ 9.4, $10 \mathrm{mM} \mathrm{DTT}$ ) and incubated for $30 \mathrm{~min}$ with $90 \mathrm{rpm}$ shaking at $30^{\circ} \mathrm{C}$. Cells were pelleted at $4,000 \mathrm{rpm}$ for $8 \mathrm{~min}$ at $18^{\circ} \mathrm{C}$, supernatant was discarded and pellet was washed with $200 \mathrm{~mL} 1.2 \mathrm{M}$ sorbitol. Cells were pelleted again and resuspended in zymolyase buffer without enzyme $(7 \mathrm{~mL} / \mathrm{g} \mathrm{cww}$, $20 \mathrm{mM} \mathrm{K} \mathrm{HPO}_{4} / \mathrm{KH}_{2} \mathrm{PO}_{4}$ buffer $\mathrm{pH} 7.4,1.2 \mathrm{M}$ sorbitol). Zymolyase was resuspended in $1 \mathrm{~mL} 1.2 \mathrm{M}$ sorbitol and added to the resuspension, incubated for $1 \mathrm{~h}$ with $90 \mathrm{rpm}$ shaking at $30^{\circ} \mathrm{C}$. Cells were pelleted at $3,000 \mathrm{rpm}$ for $10 \mathrm{~min}$ at $18^{\circ} \mathrm{C}$; the supernatant was discarded. The following steps were carried out at $4^{\circ} \mathrm{C}$. The pellet was washed with cold zymolyase buffer without enzyme. Cells were subsequently pelleted at 3,000 rpm for $10 \mathrm{~min}$ at $4^{\circ} \mathrm{C}$ and the pellet was resuspended in homogenization buffer $(7 \mathrm{~mL} / \mathrm{g} \mathrm{cww}$, $10 \mathrm{mM}$ Tris-HCl pH 7.4, 0.6 M sorbitol, 125 mM EDTA pH 8, 2 g/L BSA, 50 mM PMSF). The suspension was pottered with a dounce homogenizer on ice (15 strokes at $900 \mathrm{rpm}$ ), transferred to SS34 tubes, and centrifuged at $3,000 \mathrm{rpm}$ for $5 \mathrm{~min}$ at $4^{\circ} \mathrm{C}$. The pellet was discarded, and the supernatant was transferred to a new SS34 tube and centrifuged at $4,000 \mathrm{rpm}$ for $10 \mathrm{~min}$ at $4^{\circ} \mathrm{C}$. The pellet was again discarded, and the supernatant transferred to new tubes and centrifuged at 12,000 rpm (Avanti J-26 XP/ JA-20) for $15 \mathrm{~min}$ at $4^{\circ} \mathrm{C}$. The supernatant of this centrifugation step was discarded, and the pellet consisting of crude mitochondrial extract was washed with $5 \mathrm{~mL}$ of SEM/PMSF buffer $(250 \mathrm{mM}$ sucrose, $20 \mathrm{mM}$ MOPS pH 7.2 , $1 \mathrm{mM}$ EDTA, $1 \mathrm{mM}$ PMSF; $5 \mathrm{~mL} / \mathrm{strain}$ ) and pelleted at $12,000 \mathrm{rpm}$ for $15 \mathrm{~min}$ at $4^{\circ} \mathrm{C}$. The supernatant was discarded, and pelleted mitochondria resuspended in 500-1000 $\mu \mathrm{L}$ SEM buffer. Protein concentration was calculated by Bradford assay (Roti ${ }^{\circledR}$-Quant (Roth), manufacturer's instructions). 50 or $100 \mu \mathrm{L}$ portions of crude mitochondrial extract with a concentration of $10 \mu \mathrm{g} / \mu \mathrm{L}$ were snap frozen in liquid nitrogen and stored at $-80^{\circ} \mathrm{C}$ for further use.

\subsubsection{Tandem sucrose gradient centrifugation}

Isolated yeast mitochondria were further purified via tandem sucrose gradient centrifugation according to the protocol published by Meisinger, Pfanner and Truscott ${ }^{239}$. 
Briefly, a sucrose step gradient with $1.5 \mathrm{~mL} 60 \%$ sucrose in EM buffer (1 mM EDTA, $10 \mathrm{mM}$ MOPS-KOH pH 7.2), $4 \mathrm{~mL} 32 \%, 1.5 \mathrm{~mL} 23 \%$, and $1.5 \mathrm{~mL} 15 \%$ sucrose in EM buffer from bottom to top was prepared. The crude mitochondrial extract was loaded onto the gradient and centrifuged at $134,000 \times g, 2^{\circ} \mathrm{C}$ for $1 \mathrm{~h}$. Purified mitochondria collected at the interface between $60 \%$ and $32 \%$ sucrose. Purified mitochondria were stored at $-80^{\circ} \mathrm{C}$ for further use.

\subsubsection{Respirometry}

The Oxygraph-2k high-resolution respirometry system (Oroboros Instruments) was used to measure oxygen consumption of freshly prepared crude mitochondrial extracts, frozen crude mitochondrial extracts, freshly prepared purified mitochondria, and frozen purified mitochondria. The chambers were set to $30^{\circ} \mathrm{C}$ with a stirrer speed of $750 \mathrm{rpm}$ and data recording of $-2 \mathrm{~s}$, and were washed once with $70 \%$ ethanol for 5 min and twice with $\mathrm{H}_{2} \mathrm{O}$ for $2 \mathrm{~min}$ each. Oxygen concentration within the chambers was equilibrated with respiration buffer (225 mM sucrose, $75 \mathrm{mM}$ mannitol, $10 \mathrm{mM}$ Tris- $\mathrm{HCl} \mathrm{pH} 7.4,10 \mathrm{mM}$ $\mathrm{KH}_{2} \mathrm{PO}_{4}, 5 \mathrm{mM} \mathrm{MgCl}, 10 \mathrm{mM} \mathrm{KCl}$ ). Oxygen consumption recordings started with the sequential addition of $10 \mathrm{mM}$ pyruvate and $2 \mathrm{mM}$ malate (substrates) and either 50 or $100 \mu \mathrm{g}$ mitochondria. Maximum OXPHOS capacity was determined by adding $1 \mathrm{mM}$ ADP and, subsequently, $10 \mathrm{mM}$ succinate. Respiration was terminated by adding $5 \mu \mathrm{M}$ antimycin A, $2 \mathrm{mM}$ ascorbate and $500 \mu \mathrm{M}$ TMPD (N,N,N',N'-tetramethyl-pphenylenediamine). $100 \mathrm{mM} \mathrm{NaN}_{3}$ was added and values were subtracted from these obtained after TMPD/ascorbate incubation. For the quality control respirometry assay, $15 \mu \mathrm{M}$ oligomycin and $0.25 \mu \mathrm{M}$ carbonyl cyanide-4-(trifluoromethoxy)phenylhydrazone (FCCP) were added before terminating respiration by adding $500 \mu \mathrm{M}$ antimycin $\mathrm{A}$.

\subsubsection{Import assay}

Protein import assays were performed as described by Ryan et al. ${ }^{240}$ Considered proteins Atp2, Cox12 and Cox13 were translated in rabbit reticulocyte lysate (Promega) after in vitro transcription of amplified DNA products. During translation, proteins were radiolabeled with $\left[{ }^{35} \mathrm{~S}\right]-$ methionine. Proteins and isolated mitochondria were mixed together in import buffer (250 mM sucrose, $10 \mathrm{mM} \mathrm{MOPS} / \mathrm{KOH} \mathrm{pH} \mathrm{7.2,} 80 \mathrm{mM} \mathrm{KCl,} 2 \mathrm{mM}$ $\mathrm{KH}_{2} \mathrm{PO}_{4}, 5 \mathrm{mM} \mathrm{MgCl}_{2}, 5 \mathrm{mM}$ methionine, and $3 \%$ fatty acid-free BSA; import buffer for Cox12 without BSA), with addition of $5 \mathrm{mM}$ creatine phosphate and $0.1 \mathrm{mg} / \mathrm{mL}$ creatine kinase, $2 \mathrm{mM}$ ATP and $2 \mathrm{mM}$ NADH. Atp2 and Cox13 imports were stopped through interruption of the membrane potential via supplementation with $8 \mu \mathrm{M}$ antimycin $\mathrm{A}, 1 \mu \mathrm{M}$ 
valinomycin, and $20 \mu \mathrm{M}$ oligomycin. $50 \mathrm{mM}$ IAA supplementation was used to halt Cox12 import. Mitochondria with imported Cox12 and Cox13 were lysed in $0.6 \% n$-dodecyl $\beta$-Dmaltoside (DDM) buffer for BN-PAGE, mitochondria with imported Atp2 were lysed in Laemmli buffer for SDS-PAGE.

\subsubsection{Immunoprecipitation}

For immunoprecipitation of Cox12 and Pam18 from wild type (WT) and HA-tagged Min8 yeast cells, antisera specific for Cox12 and Pam18 were attached to protein A-sepharose (GE Healthcare), as described elsewhere ${ }^{241}$. Mitochondria that were untreated or crosslinked with BS3 were lysed with $20 \mathrm{mM}$ Tris $\mathrm{pH} 7.4,80 \mathrm{mM} \mathrm{NaCl}, 0.5 \mathrm{mM}$ EDTA, $1 \%$ digitonin, $10 \%$ glycerol and $1 \mathrm{mM}$ PMSF for $1 \mathrm{~h}$ at $4^{\circ} \mathrm{C}$ prior to immunoprecipitation. Lysates were loaded onto HA-antibody columns and incubated at $4^{\circ} \mathrm{C}$ for $1 \mathrm{~h}$. After elution with $0.1 \mathrm{M}$ glycine $\mathrm{pH} 2.8$ proteins were subjected to SDS-PAGE and Western blotting. ANTI-FLAG M2 affinity gel (Sigma-Aldrich) was used for the isolation of Min8 ${ }^{\mathrm{FLAG}}(20 \mu \mathrm{L}$ per $500 \mu \mathrm{g}$ mitochondria). $0.5 \%$ Triton X-100 and $0.1 \%$ SDS was used for solubilization and washing. Elution was performed with $0.1 \mathrm{M}$ glycine $\mathrm{pH} 2.5$ for 5 min at room temperature $(\mathrm{RT})$.

\subsubsection{Blue native-PAGE and activity staining}

Blue native-PAGE (BN-PAGE) 242,243 in combination with activity staining was performed to detect the activity of complexes II, IV and V within the respiratory chain of crude mitochondrial extract and purified mitochondria. BN-PAGE was also performed to investigate the association of Min8 to the cytochrome coxidase. Approximately $300 \mu \mathrm{g}$ of mitochondria were pelleted at $14,000 \mathrm{rpm}$ for $10 \mathrm{~min}$ at $4^{\circ} \mathrm{C}$. Pellets were resuspended in $300 \mu \mathrm{L}$ of solubilization buffer $(20 \mathrm{mM}$ Tris- $\mathrm{HCl} \mathrm{pH} \mathrm{7.4,50} \mathrm{mM} \mathrm{NaCl}, 10 \%$ glycerol, $5 \mathrm{mM}$ EDTA, $1 \mathrm{mM} \mathrm{PMSF,} 1 \%$ digitonin or $0.6 \%$ DDM), and incubated for $15 \mathrm{~min}$ on ice. After spinning at $14,000 \mathrm{rpm}$ for $15 \mathrm{~min}$ at $4^{\circ} \mathrm{C}$, the supernatant was incubated with loading dye (5 \% Coomassie G-250, 500 mM 6-amino-hexanoic acid and 0.1 M Bis-Tris, $\mathrm{pH}$ 7.0) for $3 \mathrm{~min}$ on ice. Again, samples were spun down at $14,000 \mathrm{rpm}$ for $2 \mathrm{~min}$ at $4^{\circ} \mathrm{C}$ and the supernatant was loaded onto a $4-10 \%$ or $6-10 \%$ BN gel. $600 \mathrm{~V}, 15 \mathrm{~mA}$ were applied for $4 \mathrm{~h}$ to separate protein complexes. After separation, proteins were stained with Coomassie. For complex II activity staining, the gel was equilibrated in $30 \mathrm{~mL}$ of $5 \mathrm{mM}$ Tris- $\mathrm{HCl} \mathrm{pH} 7.4$ for $30 \mathrm{~min}$ and incubated with $75 \mathrm{mg}$ nitro blue tetrazolium chloride (NBT) in Tris- $\mathrm{HCl}$ and $3 \mathrm{mg} \mathrm{NADH}$ at $4^{\circ} \mathrm{C}$ overnight. For complex IV activity staining, the gel was equilibrated in $30 \mathrm{~mL}$ of $50 \mathrm{mM} \mathrm{K} \mathrm{HPO}_{4} / \mathrm{KH}_{2} \mathrm{PO}_{4} \mathrm{pH} 7.2$ for 30 min and incubated with 
$15 \mathrm{mg} \mathrm{3,3'-diaminobenzidine}(\mathrm{DAB})$ and $3 \mathrm{mg}$ reduced cytochrome $c$ at $30^{\circ} \mathrm{C}$ overnight. For complex $V$ activity staining, the gel was equilibrated with $30 \mathrm{~mL}$ TG buffer ( $35 \mathrm{mM}$ Tris$\mathrm{HCl}, 220 \mathrm{mM}$ glycine, $\mathrm{pH}$ 8.3) for $30 \mathrm{~min}$ and incubated with $0.5 \mathrm{mM} \mathrm{ATP} 1 \mathrm{M} \mathrm{MgSO}_{4}$ and $0.01 \% \mathrm{~Pb}\left(\mathrm{NO}_{3}\right)_{2}(\mathrm{w} / \mathrm{v})$ for $5-12 \mathrm{~h}$.

\subsubsection{Western blotting and immunodetection}

Standard protocols were followed for Western blotting and immunodetection ${ }^{244}$. PVDF membranes (Immobilon-P, Merck Millipore) were methanol-activated and, together with blotting paper and the gel, wetted with transfer buffer (20 mM Tris, $150 \mathrm{mM}$ glycine, $0.02 \%$ SDS, $20 \%$ ethanol) for semi-dry Western blotting. Blotting was performed at $25 \mathrm{~V}$ and $250 \mathrm{~mA}$ for 2.5-3 h. Membranes were blocked with 5-10\% milk powder/TBS-T (20 mM Tris pH 7.5, $62 \mathrm{mM} \mathrm{NaCl}, 0.1 \%$ Tween 20) for $1 \mathrm{~h}$ at RT. Membranes were incubated for 1-2 $\mathrm{h}$ at RT with primary antibodies diluted in $5 \%$ milk powder/TBS-T. After rinsing the membranes thrice with TBS-T for $10 \mathrm{~min}$, secondary antibodies diluted in $5 \%$ milk powder/TBS-T were incubated for $1 \mathrm{~h}$ at RT. Depending on the specificities of the secondary antibodies, the readout was performed via horseradish peroxidase assay or via fluorescent dye.

\subsubsection{Proteinase $\mathrm{K}$ assay}

Proteinase $\mathrm{K}(\mathrm{PK})$ assay was performed to check mitochondrial membrane integrity after swelling in slightly hypotonic cross-linking buffer (20 mM HEPES pH 7.5, $100 \mathrm{mM} \mathrm{NaCl}$ ). $750 \mu \mathrm{g}$ of purified mitochondria were thawed, pelleted at $10,000 \mathrm{xg}$ for $10 \mathrm{~min}$ at $4^{\circ} \mathrm{C}$ and resuspended in cross-linking buffer. One sample of mitochondria was treated with 0.2 or $1 \mu \mathrm{g} \mathrm{PK} / \mu \mathrm{g}$ mitochondria, and a control sample without PK, both incubated for $1 \mathrm{~h}$ at RT. To fully access matrix proteins, another control sample was treated with $5 \%$ DMSO and by sonication in a bath (10 pulses, 5 cycles) before adding PK. PMSF was added to a final concentration of $4 \mathrm{mM}$ and incubated for $10 \mathrm{~min}$ on ice. Samples were subjected to SDSPAGE. Proteins were separated with $250 \mathrm{~V}, 30 \mathrm{~mA}$ for $2.5 \mathrm{~h}$ and subsequently blotted. Identification was performed with antibodies against Tom70, Tim44, Tim21, Cor1, Rcf2, Rcf3, Mia40, Por1 and Cox12. All indicated antibodies are polyclonal and were raised in rabbit (Gramsch Laboratories, Schwabhausen, Germany) against C-terminal peptides, recombinant whole protein or recombinant protein domains. 


\subsubsection{HEK293T cell culture}

Human embryonic kidney cells (HEK293-Flp-In T-Rex; HEK293T) were cultured as described elsewhere ${ }^{245}$. Briefly, cells were grown in Dulbecco's modified Eagle's medium (DMEM), $10 \%$ fetal bovine serum (FBS), $2 \mathrm{mM} \mathrm{L-glutamine,} 1 \mathrm{mM}$ sodium pyruvate, $50 \mu \mathrm{g} / \mathrm{mL}$ uridine, $100 \mathrm{units} / \mathrm{mL}$ penicillin, and $100 \mu \mathrm{g} / \mathrm{mL}$ streptomycin, at $37^{\circ} \mathrm{C}$ and $5 \%$ $\mathrm{CO}_{2}$.

\subsubsection{Isolation of human mitochondria and generation of mitoplasts}

Human mitochondria were isolated as described elsewhere ${ }^{246}$. Briefly, cells were resuspended in homogenization buffer $(300 \mathrm{mM}$ trehalose, $10 \mathrm{mM} \mathrm{KCl}, 10 \mathrm{mM}$ HEPES $\mathrm{pH} 7.4$ ) containing $1 \mathrm{mM}$ PMSF and $0.2 \% \mathrm{BSA}$, and subsequently homogenized with Homogen ${ }^{\text {plus }}$ Homogenizer (Schuett-Biotec, Germany). Mitochondria were isolated via differential centrifugation and pelleted at 11,000xg for $10 \mathrm{~min}$, washed with homogenization buffer and again centrifuged. Mitoplasts were generated as described elsewhere ${ }^{245}$. Isolated mitochondria were resuspended in homogenization buffer containing $0.1 \%$ digitonin for $30 \mathrm{~min}$ and treated with proteinase $\mathrm{K}$ for $15 \mathrm{~min}(0.5 \mu \mathrm{g}$ per $100 \mu \mathrm{g}$ of mitochondria). Proteinase $\mathrm{K}$ digestion was terminated by adding $2 \mathrm{mM}$ PMSF and resulting mitoplasts were washed seven times with homogenization buffer prior to cross-linking procedure (twice with addition of $2 \mathrm{mM} \mathrm{PMSF}$ and $0.2 \%$ BSA, twice with $2 \mathrm{mM}$ PMSF and 3 times without any supplements).

\subsubsection{Cross-linking of purified yeast mitochondria, lysis and protein precipitation}

Cross-linking reactions were performed as described in Linden et al. ${ }^{247}$. Aliquots of purified mitochondria of each condition were stored in $1 \mathrm{mg}$ portions at $-80^{\circ} \mathrm{C}$. Mitochondria were thawed and pelleted at $10,000 \mathrm{xg}$ for $5 \mathrm{~min}$ at $4^{\circ} \mathrm{C}$. Pelleted mitochondria were resuspended in $200 \mu \mathrm{L}$ of cross-linking buffer (20 mM HEPES pH 7.5, $100 \mathrm{mM} \mathrm{NaCl}$ ) and subsequently cross-linked with $5 \mathrm{mM}$ bis(sulfosuccinimidyl)suberate (BS3, Thermo Fisher Scientific), incubated for $1 \mathrm{~h}$ at RT. The reaction was quenched with a final concentration of $50 \mathrm{mM}$ Tris $\mathrm{pH}$ 8, incubated for $15 \mathrm{~min}$ at RT. Cross-linked mitochondria were lysed with $2 \%$ SDS and boiled at $70^{\circ} \mathrm{C}$ for $10 \mathrm{~min}$. Proteins were precipitated by incubation at $-20^{\circ} \mathrm{C}$ overnight with four times the sample volume of ice-cold acetone.

Cross-linking reactions for the comparison of BS3 with disuccinimidyl suberate (DSS) were performed with the following changes: freshly prepared crude mitochondrial 
extract was cross-linked with an equimolar mixture of stable-isotopically labeled BS3-d4 (resuspended in cross-linking buffer) and DSS (resuspended in DMSO, both Thermo Fisher Scientific). The final concentration of each cross-linker was $1 \mathrm{mM}$.

Cross-linking reactions for the quantitative experiments were performed with the following changes: $0.5 \mathrm{mg}$ of mitochondria derived from yeast grown on glycerol- or on glucose-containing medium, respectively, were cross-linked with either $5 \mathrm{mM} \mathrm{BS3}$ or $5 \mathrm{mM}$ stable-isotopically labeled BS3-d4 in a label-swap experiment. After quenching, samples were equally combined based on their protein amount (see subsection 2.2.17). For lysis, $8 \mathrm{M}$ urea was used in combination with sonication (diagenode Bioruptor, $3 \times 30 \mathrm{~s}$ at full power, $\left.4^{\circ} \mathrm{C}\right)$. Proteins were not precipitated.

\subsubsection{Cross-linking of human mitoplasts, lysis and protein precipitation}

Human mitoplasts (see subsection 2.2.12) were aliquoted in $1 \mathrm{mg}$ portions. Pellets were resuspended in $0.5 \mathrm{~mL}$ cross-linking buffer (20 mM HEPES, $\mathrm{pH} 7.5,100 \mathrm{mM} \mathrm{NaCl}$ ). Mitoplasts were cross-linked with either $5 \mathrm{mM}$ BS3 or $5 \mathrm{mM}$ 1-ethyl-3-(3dimethylaminopropyl)carbodiimide hydrochloride (EDC) in combination with $10 \mathrm{mM} \mathrm{N}$ hydroxysulfosuccinimide (sulfo-NHS) for $45 \mathrm{~min}$ at RT. Reactions were quenched, and mitoplasts were pelleted at $12,000 \times \mathrm{xg}$ for $10 \mathrm{~min}$ at $4^{\circ} \mathrm{C}$ for subsequent lysis. Lysis was

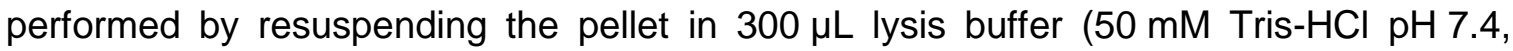
$100 \mathrm{mM} \mathrm{NH}_{4} \mathrm{Cl}, 10 \mathrm{mM} \mathrm{MgCl}_{2}, 2 \%$ SDS; $20 \mathrm{mM}$ DTT for EDC cross-linking), followed by a short incubation of $10 \mathrm{~min}$ at RT with gentle shaking. Samples were spun down at $16,000 \times \mathrm{g}$ for $20 \mathrm{~min}$ at $\mathrm{RT}$, and the proteins in the supernatant were precipitated by adding ice-cold acetone at a ratio of four times the sample volume, followed by overnight incubation at $-20^{\circ} \mathrm{C}$. After precipitation, samples were spun down at $10,000 \times \mathrm{g}$ for $5 \mathrm{~min}$ at $4^{\circ} \mathrm{C}$. Pellets were resuspended with $1 \mathrm{~mL}$ of ice-cold $80 \%$ ethanol, spun down again and air dried.

\subsubsection{5. $\mathrm{CuSO}_{4}$ cross-linking}

Cysteine cross-linking induced by $\mathrm{CuSO}_{4}$ was performed as described by Kobashi248 with the following changes: Mitochondria were resuspended in $\mathrm{SH}$ buffer $(0.6 \mathrm{M}$ sorbitol, $20 \mathrm{mM}$ HEPES $\mathrm{pH}$ 7.5) and incubated with $2 \mathrm{mM} \mathrm{CuSO}_{4}$ for $30 \mathrm{~min}$ on ice. Quenching was performed by adding $17 \mathrm{mM} N$-ethylmaleimide (NEM) and $17 \mathrm{mM}$ EDTA, incubated for $15 \mathrm{~min}$ on ice. 


\subsubsection{Protein size exclusion chromatography}

For one biological replicate of cross-linked mitochondria derived from yeast grown on glycerol medium, proteins were prefractionated by protein size exclusion chromatography (protein SEC) after lysis. Precipitated proteins were resuspended in $100 \mu \mathrm{L}$ of buffer containing $20 \mathrm{mM}$ HEPES $\mathrm{pH} 8,100 \mathrm{mM} \mathrm{NaCl}$ and $1 \%$ SDS. Cross-linked protein complexes were separated by loading them onto a Superose-6 Increase 3.2/300 column coupled to an ÄKTAmicro system (both GE Healthcare). Protein SEC running buffer consisted of $20 \mathrm{mM}$ HEPES $\mathrm{pH} 8,100 \mathrm{mM} \mathrm{NaCl}$ and $0.1 \%$ SDS. The system was operated with a flow rate of $40 \mu \mathrm{L} / \mathrm{min}$ and fractions with a volume of $100 \mu \mathrm{L}$ were collected. Proteins within each fraction were further subjected to in-solution digestion (see subsection 2.3.1).

\subsubsection{BCA assay}

Protein concentration was estimated using the Pierce ${ }^{\mathrm{TM}}$ BCA Protein Assay Kit (Thermo Fisher Scientific). Bovine serum albumin (BSA) serial dilutions in a working range of 25$2,000 \mu \mathrm{g} / \mathrm{mL}$ were used to build a standard curve. $5 \mu \mathrm{L}$ of each sample or standards were mixed with $100 \mu \mathrm{L}$ of $\mathrm{BCA}$ working reagent (solution $\mathrm{A}$ and solution $\mathrm{B}$ freshly mixed in a $50: 1$ ratio) and incubated for $30 \mathrm{~min}$ at $37^{\circ} \mathrm{C}$. Absorbance values measured at $562 \mathrm{~nm}$ were used to determine protein concentration. Values in samples with unknown protein concentration were estimated by inference from a BSA standard curve.

\subsection{Mass spectrometry and proteomics methods}

\subsubsection{Protein digestion in-solution}

Precipitated proteins were resuspended in $50 \mu \mathrm{L} 8 \mathrm{M}$ urea/50 mM ammonium bicarbonate $(A B C) \mathrm{pH}$ 8. For proteins derived from human mitoplasts, an additional sonication step (diagenode Bioruptor, $3 \times 30 \mathrm{~s}, 4^{\circ} \mathrm{C}$ ) was performed. Proteins were reduced by adding DTT to a final concentration of $10 \mathrm{mM}$, incubated for $1 \mathrm{~h}$ at RT. Subsequently, proteins were alkylated by adding iodoacetamide (IAA) to a final concentration of $40 \mathrm{mM}$, incubated for 30 min at RT in the dark. Proteins were digested by trypsin (Promega) in an enzyme-toprotein ratio of $1: 50$ overnight at $37^{\circ} \mathrm{C}$ (see ref $\mathrm{f}^{249}$ for protocol overview). The digestion was stopped by adding trifluoroacetic acid (TFA) to a final concentration of $0.5 \%$. Peptides were then subjected to desalting (see subsection 2.3.4). 


\subsubsection{SDS-PAGE}

After digesting proteins derived from cross-linked human mitoplasts, the resulting precipitation after acidifying to $0.5 \%$ TFA was resuspended in 4X NuPAGE LDS Sample Buffer with reducing agent (106 mM Tris- $\mathrm{HCl}, 141 \mathrm{mM}$ Tris-Base, $2 \%$ LDS, $10 \%$ glycerol, $0.51 \mathrm{mM}$ EDTA, $0.22 \mathrm{mM}$ SERVA Blue G250, $0.175 \mathrm{mM}$ Phenol Red, $50 \mathrm{mM}$ DTT; Thermo Fisher Scientific), diluted to $1 \mathrm{X}$ and heated up at $70^{\circ} \mathrm{C}$ for $10 \mathrm{~min}$. Solubilized proteins were separated by SDS-polyacrylamide gel electrophoresis (PAGE) 250 by loading them to NuPAGE 4-12\% Bis-Tris gels (Thermo Fisher Scientific), applying $200 \mathrm{~V}$ for $45 \mathrm{~min}$. Gels were stained with Coomassie InstantBlue (Merck) for $2 \mathrm{~h}$ and destained with deionized water overnight. Proteins were then in-gel digested (see subsection 2.3.3).

\subsubsection{Protein digestion in-gel}

In-gel protein digestion was performed as initially described elsewhere ${ }^{251}$ with a few changes. Briefly, SDS-PAGE lanes were cut into 23 equal-sized slices and chopped into small pieces. Pieces were hydrated with $150 \mu \mathrm{L} \mathrm{H}_{2} \mathrm{O}$ for 5 min at RT with gentle shaking. After removal of the liquid, gel pieces were shrunk by adding $150 \mu \mathrm{L} \mathrm{ACN}$, incubated for $15 \mathrm{~min}$. Proteins within the gel pieces were reduced by adding $150 \mu \mathrm{L} 10 \mathrm{mM}$ $\mathrm{DTT} / 100 \mathrm{mM} \mathrm{ABC}$, incubated for $50 \mathrm{~min}$ at $56^{\circ} \mathrm{C}$. Again, pieces were shrunk by adding ACN. Afterwards, proteins were alkylated by adding $150 \mu \mathrm{L} 55 \mathrm{mM}$ IAA/100 mM ABC, incubated for $20 \mathrm{~min}$ at RT in the dark. Gel pieces were washed with $150 \mu \mathrm{L} 100 \mathrm{mM} \mathrm{ABC}$ and $50 \% A C N$, incubated for $15 \mathrm{~min}$ at RT, respectively. Gel pieces were shrunk again with $\mathrm{ACN}$ and air dried. $30 \mu \mathrm{L}$ trypsin solution (1 $\mu \mathrm{g}$ trypsin (Sigma), $5 \mathrm{mM} \mathrm{CaCl}_{2}, 50 \mathrm{mM}$ $A B C$ ) were added to the gel pieces and incubated for $10 \mathrm{~min}$ at $4^{\circ} \mathrm{C}$. Gel pieces were covered with additional $30 \mu \mathrm{L} 5 \mathrm{mM} \mathrm{CaCl}_{2} / 50 \mathrm{mM} \mathrm{ABC}$. Trypsin (Sigma-Aldrich) digestion was performed at $37^{\circ} \mathrm{C}$ overnight. After digestion, gel pieces were shrunk by adding $80 \mu \mathrm{L}$ $A C N$, incubated for $15 \mathrm{~min}$ at $37^{\circ} \mathrm{C}$. From this point, supernatants containing peptides were collected. Peptides retained in the gel pieces were acidified by adding $80 \mu \mathrm{L} 5 \%$ formic acid, incubated for $15 \mathrm{~min}$ at $37^{\circ} \mathrm{C}$. Same volume of $\mathrm{ACN}$ was added before an additional incubation round for $15 \mathrm{~min}$ at $37^{\circ} \mathrm{C}$. Supernatants were pooled, vacuum dried and subjected to LC-MS/MS analysis (see subsection 2.3.6).

\subsubsection{Peptide desalting}

SepPak cartridges (1cc, tC18, Waters) were used for peptide desalting, an upscaled and modified version of the method described earlier ${ }^{252}$. C18 material was activated with $1 \mathrm{~mL}$ 
$100 \%$ ACN followed by $1 \mathrm{~mL} 80 \%$ ACN/0.1\% TFA and three times $1 \mathrm{~mL} 0.1 \%$ TFA. Peptides were loaded onto the cartridge and pushed through by gravity flow. Bound peptides were washed twice with $1 \mathrm{~mL} 0.1 \%$ TFA and finally eluted twice with $500 \mu \mathrm{L}$ of $80 \%$ ACN $/ 0.1 \%$ TFA. Combined eluates were vacuum dried in a vacuum concentrator.

\subsubsection{Enrichment of cross-linked peptides by size exclusion chromatography}

For enrichment of cross-linked peptides, peptide size exclusion chromatography (peptide SEC) was performed ${ }^{214}$. Dried peptides were resuspended in peptide SEC running buffer (30\% ACN/0.1\% TFA). A SuperdexPeptide 3.2/300 column (GE Healthcare) connected to an Agilent $1100 \mathrm{HPLC}$ was used for separation. A flow rate of $50 \mu \mathrm{L} / \mathrm{min}$ was applied and fractions with a volume of $50 \mu \mathrm{L}$ were collected. Fractions between 1.1 and $1.4 \mathrm{~mL}$ column volume were vacuum dried, resuspended in mass spectrometry loading buffer (2$4 \%$ ACN/0.05 \% TFA) and analyzed by LC-MS/MS (see subsection 2.3.6).

\subsubsection{LC-MS/MS data acquisition}

LC-MS/MS data were acquired according to Linden et al. ${ }^{247}$ Fractions derived from peptide SEC that contained cross-linked peptides were measured twice on an Orbitrap Fusion or Orbitrap Fusion Lumos Tribrid Mass Spectrometer (Thermo Fisher Scientific). Mass spectrometers were coupled online to a Dionex UltiMate 3000 UHPLC system (Thermo Fisher Scientific) equipped with an in house-packed C18 column (ReproSil-Pur 120 C18$A Q, 1.9 \mu \mathrm{m}$ pore size, $75 \mu \mathrm{m}$ inner diameter, $30 \mathrm{~cm}$ length, Dr. Maisch $\mathrm{GmbH})$. The following $180 \mathrm{~min}$ LC method with a flow rate of $300 \mathrm{~nL} / \mathrm{min}$ was applied for peptide separation: mobile phase $A$ consisted of $0.1 \%$ formic acid $(F A, v / v)$, mobile phase $B$ consisted of $80 \% A C N / 0.08 \%$ FA (v/v). The method started at $5 \% \mathrm{~B}$, increasing to 10 $20 \%$ B within 3 min (depending on the peptide SEC fraction; for earlier fractions a steeper start point was chosen), followed by an increase to $46 \%$ B within $168 \mathrm{~min}$, then keeping B constant at $90 \%$ for $6 \mathrm{~min}$. The column was equilibrated to $5 \% \mathrm{~B}$ for $6 \mathrm{~min}$. The orbitrap (OT) mass detector was used for MS1 and MS2 spectra acquisition with a resolution of 120,000 and 30,000, respectively. In MS1, the scan range was set from 350 to $1550 \mathrm{~m} / \mathrm{z}$, automatic gain control (AGC) target to $5 \times 10^{5}$ and the maximum injection time (IT) to $60 \mathrm{~ms}$. The dynamic exclusion time was set to $10 \mathrm{~s}$. For fragmentation, the 20 most abundant precursors with a charge state between 3-8 were selected per duty cycle. Higher-energy collisional dissociation (HCD) with a normalized collision energy of $30 \%$ was applied for precursor fragmentation. For MS2 spectra acquisition, the AGC target was set to $5 \times 10^{4}$ 
and IT to $128 \mathrm{~ms}$. These settings were also applied for the quantitative comparison of BS3d4 and DSS. Here, one technical replicate was measured on Orbitrap Fusion Lumos.

For the quantitative experiments and the analysis of peptides derived from human mitoplasts, the following changes were applied: peptides were analyzed on a $Q$ Exactive HF-X mass spectrometer (Thermo Fisher Scientific). For MS1, AGC target was set to $1 \times 10^{6}$ and IT to $50 \mathrm{~ms}$. Dynamic exclusion was set to $60 \mathrm{~s}$ and for fragmentation the 30 most abundant precursor ions were selected per duty cycle. For MS2, AGC target was set to $1 \times 10^{5}$.

\subsection{Data processing and analysis}

\subsubsection{Analysis of data acquired from cross-linked yeast mitochondria}

\subsubsection{Generation of the databases}

Data were analyzed according to Linden et al. ${ }^{247}$ Proteome Discoverer (v. 2.1, Thermo Fisher Scientific) was used to convert .raw files to .mgf files. For this, the signal-to-noise ratio was set to 1.5 and the considered precursor mass to $1,000-10,000 \mathrm{Da}$. Based on these converted data, dedicated, biological replicate-specific databases containing the 400 most abundant proteins (top400) were created. For this, proteins were identified based on their linear, i.e. non-cross-linked, peptides by $\operatorname{Mascot}^{167}$ (v. 2.3.02) and sorted in Scaffold ${ }^{253}$ (v. 4) by dividing total spectral counts by the molecular weight of the specific proteins. In Mascot, the following settings were applied: precursor tolerance was set to 10 ppm, fragment tolerance to $0.02 \mathrm{Da}$. Trypsin was selected as protease, with maximum four missed cleavage sites. Carbamidomethylation on cysteines, oxidation of methionines, hydrolyzed BS3 (mass shift $+156.077 \mathrm{Da}$ ) and Tris-quenched BS3 (mass shift +259.142 Da) were set as variable modifications. False discovery rate (FDR) was set to $1 \%$. A reviewed yeast database (UniProt/SwissProt, 02/2016, 23481 entries) was used for the search. Finally, proteins originated from S. cerevisiae were selected.

Databases used for the quantitative experiments were generated by using MaxQuant ${ }^{254}$ (v. 1.6.0.1). Compared to the Mascot search described above, the following changes were applied: for the search, .raw files were used; MaxQuant was operated in default mode with above mentioned BS3-modifications added; trypsin was allowed to have maximum three missed cleavage sites; the reviewed $S$. cerevisiae database (UniProt/SwissProt, 12/2016, 6721 entries) was used. To rank identified proteins, iBAQ values were taken into consideration. Generated databases were specific for one labelswap experiment. 


\subsubsection{Identification of cross-linked peptides}

All converted .mgf files from both the qualitative and quantitative experiments were analyzed by pLink $1^{255,256}$ (v. 1.23) with the following settings: BS3 was set as cross-linker, BS3-d4 was additionally considered for the quantitative experiments; trypsin was selected as protease with maximum two missed cleavage sites; carbamidomethylation on cysteines was defined as fixed modification, oxidation of methionines was set as variable modification; precursor mass tolerance was set to $\pm 5 \mathrm{Da}$ and was adapted to $\pm 4 \mathrm{Da}$ for analysis of quantitative data. A mass filter of $\pm 10 \mathrm{ppm}$ was applied to every selected isotopic peak. Fragment ion mass tolerance was set to $20 \mathrm{ppm}$. $1 \%$ FDR was applied at spectrum level.

\subsubsection{Quantitation of cross-linked peptides}

Cross-linked peptides identified by pLink 1 were quantified by $\mathrm{XiQ}^{226}$, operated with default settings. Quantitation of peptides was based on areas under the curve taken from .raw files. Areas under the curve were summed up for the first to the third isotopic peak separately for heavy-labeled and light peptides. Signals with a drop to $10 \%$ of intensity were considered and monoisotopic peaks were excluded from the calculation to avoid an overlap between labeled and unlabeled peptides. Heavy-to-light ratios of peptides were finally calculated by dividing the summed areas of the corresponding peaks. Ratios were then $\log _{2}$-transformed and median-normalized. An in-house written $R$ script provided by Dr. Iwan Parfentev was used to condense quantitative information to unique peptide pairs by a weighted intensity average of differently charged precursor ions corresponding to the same peptide (according to Chen et al. ${ }^{257}$ ).

\subsubsection{Analysis of data acquired from cross-linked human mitoplasts}

\subsubsection{Generation of the databases}

Proteins were identified based on their linear peptides by MaxQuant (v. 1.6.0.1). Same settings as described above were applied with the following changes: for the EDC data set, hydrolyzed and Tris-quenched BS3 modifications were exchanged by Tris-quenched EDC modification (mass shift $+91.063 \mathrm{Da}$ ). All identified proteins were included in the database for the cross-linked peptide search (approximately 1500 to 1900 proteins). 


\subsubsection{Identification of cross-linked peptides}

For the identification of cross-linked peptides derived from human mitoplasts, pLink $2^{258}$ (v. 2.3) was used. The acquired .raw files were loaded into pLink 2 and the following settings were applied: BS3 and EDC-DE was selected as cross-linker, respectively, with the corresponding databases; trypsin was set as protease with maximum three missed cleavage sites; peptide mass was set to 400-10,000 Da and peptide length to 4-60 amino acids; precursor and fragment tolerances were set to 10 and $20 \mathrm{ppm}$, respectively; carbamidomethylation of cysteines was set as fixed modification, oxidation of methionines as variable modification. A global FDR of $1 \%$ was applied.

\subsubsection{Docking experiments}

Docking experiments were performed according to Linden et al. ${ }^{247}$ For this, the Rosetta software ${ }^{259-261}$ was used. The structure of the yeast $\mathrm{CIII}_{2} \mathrm{CIV}_{2}$ (PDB: $6 \mathrm{HU9}{ }^{91}$ ) were downloaded from the Protein Data Bank of Transmembrane Proteins (PDBTM ${ }^{262}$ ). These structures provided transformed coordinates that are appropriate for docking experiments in the membrane bilayer. Tools such as mp_span_from_pdb, mp_dock_setup (Rosetta) were used to generate the membrane topology files from the atomic models. According to the cross-linking results, docking partners were preoriented manually in PyMOL prior to actual docking to avoid global and low-resolution docking steps. Thus, docking experiments were performed using the local docking approach (high-resolution docking). Constraints provided by cross-linking results were applied in the form of "AtomPair" harmonic distances $(9.0 \AA \pm 2.5 \AA$ ) between the cross-linked atoms (NZ of lysine residues and $\mathrm{N}$ of the $\mathrm{N}$-terminal residue). For each docking experiment at least 5000 decoys were generated. These decoys were ranked based on the interface score (I_sc) representing the energy of the interactions across the interface and were subsequently clustered.

The structure of Ndi1 was taken from type-II mitochondrial NADH dehydrogenase (PDB: 4G73 ${ }^{263}$ ) and the structure of Pet9 from the mitochondrial ADP/ATP carrier protein (PDB: 4C9G ${ }^{264}$ ). Rosetta's ab initio protocol for de novo folding of membrane proteins ${ }^{265,266}$ was used to calculate the atomic model of Min8. OCTOPUS server ${ }^{267}$ as well as Robetta server (www.robetta.bakerlab.org) were used to generate the topology of transmembrane region and fragment files. Over 300,000 low-resolution centroid models were created. The 5000 most energetically favored ones were picked for clustering and converted to "fullatom" models. $\operatorname{Coot}^{268}$ were used to manually optimize the orientations of intermembrane space and matrix domains of the docked Min8 model. 


\subsubsection{General statistics}

Significance of differences between protein-protein cross-links identified in mitochondria derived from yeast grown on glycerol- or glucose-containing medium (represented in subsection 3.1.3, Figure 7) were calculated with a Chi-square test of independence. Absolute numbers of unique protein-protein cross-links (including intraprotein and interprotein cross-links) were considered. All "ambig"- and "not mt"-pairs were combined, OM-M-pairs were combined with OM-IM-pairs, and both IMS-M-pairs and IMS-IM-pairs were combined with IMS-IMS-pairs to increase the number of values per category $\geq 5$, suitable to conduct a Chi-square test of independence. Values for $\mathrm{X}^{2}$ below an alpha level of 0.05 were considered as significant. For the quantitative comparison of residue-toresidue cross-links resulting from cross-linking of mitochondria derived from yeast grown on glycerol- or glucose-containing medium, a one-sample $t$ test was applied to test for significant differences. A minimum of three valid values out of four replicates was considered. Differences with a $p$ value $\leq 0.05$ and a fold change $\geq 2$ were considered significant (see subsection 3.1.6, Figure 11).

\subsubsection{Visualization of cross-linking results}

For the visualization of protein-protein cross-links, Cytoscape ${ }^{269}, \mathrm{xiNET}^{270}$ and $x \mathrm{Vis}^{271}$ were used. Perseus ${ }^{272}$ was used to perform and display the volcano plot and principal component analysis (PCA). DisVis ${ }^{273}$ was used for the calculation of accessible interaction space. Protein structures were illustrated with PyMOL (www.pymol.org, Schrödinger LLC) and UCSF Chimera ${ }^{274}$ in combination with Xlink Analyzer ${ }^{275}$. 


\section{Results}

Results obtained during the course of this thesis are presented in this section. Chemical cross-linking in combination with mass spectrometry (XL-MS) was applied to mitochondria derived from Saccharomyces cerevisiae grown on glycerol- or glucose-containing medium. Identified protein-protein cross-links in both conditions were compared to each other in a qualitative and a quantitative approach (see section 3.1). Also, mitochondria derived from a $r 0^{0}$ yeast strain that lacks mitochondrial DNA (mtDNA) were analyzed with $\mathrm{XL}-\mathrm{MS}$ (see section 3.2). An additional protein prefractionation step was tested with crosslinked mitochondria derived from yeast grown on glycerol-containing medium to increase the number of cross-links identified by XL-MS (see section 3.3). Data obtained from crosslinked mitochondria from all strains and conditions, i.e. from yeast grown on glycerol- or glucose-containing medium and from the $r h o^{\circ}$ yeast strain, were also analyzed with a proteomics workflow identifying linear, i.e. non-cross-linked, peptides and compared to each other (see section 3.4). Prior to the cross-linking experiments, mitochondria from yeast grown on glycerol-medium were tested for membrane integrity and respiration activity (see section 3.5). Furthermore, mitoplasts generated from human mitochondria of HEK293T cells were cross-linked separately with two cross-linkers harboring different reaction chemistries for the analysis of protein-protein cross-links by XL-MS (see section 3.6). All raw files that were acquired and used for data analysis are listed in

\section{Supplementary Tables 8-15.}

\subsection{Cross-linking combined with mass spectrometry of mitochondria derived from yeast grown on glycerol- or glucose-containing medium}

In the following subsections, the results of the mass spectrometric analysis of cross-linked mitochondria derived from $S$. cerevisiae grown on glycerol- or glucose-containing medium will be presented and compared to each other. The major part of these results was published in the peer-reviewed journal Molecular \& Cellular Proteomics (Linden et al. ${ }^{247}$ ). Yeast mitochondria from both conditions were isolated and purified by Dr. Markus Deckers and Mirjam Wissel, Cellular Biochemistry, University Medical Center Göttingen. Crosslinking reactions and data acquisition of mitochondria derived from yeast grown on glucose medium and the first biological replicate of mitochondria derived from yeast grown on glycerol medium included in the qualitative data sets were performed by Dr. Chung-Tien Lee during his time as a postdoctoral researcher in the Bioanalytical Mass Spectrometry Group at the Max Planck Institute for Biophysical Chemistry. Data of the second biological replicate of cross-linked mitochondria derived from yeast grown on glycerol medium 
included in the qualitative data sets were acquired in collaboration with Dr. Iwan Parfentev, Bioanalytical Mass Spectrometry Group, Max Planck Institute for Biophysical Chemistry. Dr. Parfentev also helped in analyzing the quantitative data with fruitful discussions and with providing a self-written $\mathrm{R}$ script facilitating the analysis. Biochemical experiments regarding the analysis of Min8 and its association with the cytochrome $c$ oxidase such as BN-PAGE experiments, $\mathrm{CuSO}_{4}$ cross-linking, immunoprecipitations, related SDS-PAGE and Western blot experiments, respirometry experiments, and import assays were performed and analyzed by Dr. Markus Deckers and Bettina Homberg, Cellular Biochemistry, University Medical Center Göttingen. This is also described in more detail in Bettina Homberg's PhD thesis with the preliminary title 'Regulation of mitochondrial supercomplex formation'. Bettina Homberg is a PhD student in the GGNB program 'Molecular Medicine'. Docking experiments including Min8 and Ndi1 were performed by Dr. Piotr Neumann, Molecular Structural Biology, Institute for Microbiology and Genetics, Göttingen Center for Molecular Biosciences, Georg-August-University Göttingen. Dr. Neumann also assisted in figure preparation related to the docking experiments. Dr. Ralf Pflanz, Bioanalytical Mass Spectrometry Group, Max Planck Institute for Biophysical Chemistry, assisted in figure preparation of ETC circles.

\subsubsection{Determination of a suitable cross-linker to cross-link mitochondrial proteins}

To determine which cross-linker suits best for chemical protein-protein cross-linking within mitochondria, two commonly used ingredients were tested. For this, disuccinimidyl suberate (DSS), a membrane-permeable but water-insoluble cross-linker, and bis(sulfosuccinimidyl)suberate (BS3), a membrane-impermeable but water-soluble crosslinker were considered. An equimolar mixture of DSS and isotopically labeled BS3-d4 was added to a freshly prepared crude extract of mitochondria isolated from yeast grown on glycerol medium (see subsection 2.2.3). Mitochondria were lysed, and proteins were precipitated and tryptically digested (see subsections 2.2.13 and 2.3.1). Cross-linked peptides were enriched by peptide size exclusion chromatography ${ }^{214}$ (peptide SEC, see subsection 2.3.5) and the first fractions containing the cross-linked peptides were analyzed by liquid chromatography tandem mass spectrometry (LC-MS/MS, see subsection 2.3.6). Identification of cross-linked peptides was performed by pLink $1^{255,256}$ (v. 1.23, see subsection 2.4.1) with the 400 most abundant proteins in the database (see subsection 2.4.1.1). In total, 2028 cross-linked peptides spectrum matches (CSMs) were identified, of which 921 could be assigned to peptides cross-linked by DSS and 1107 by BS3-d4. Next, cross-links were quantified by $\mathrm{XiQ}^{226}$ (see subsection 2.4.1.3) and the suborganellar 
localization of cross-linked proteins within mitochondria were determined. Since most of the ratios of quantified residue-to-residue cross-links (amino acid of peptide A cross-linked to amino acid of peptide B) remained unchanged, the residue-to-residue cross-links with a fold change $\geq 2$ were investigated in more detail. $71-73 \%$ of the identified protein-protein cross-links to which the identified residue-to-residue cross-links infer localize either to the inner membrane (IM) or to the matrix (M), regardless of the used cross-linker (Figure 5). This indicates that also the membrane-impermeable cross-linker BS3 connects proteins of all mitochondrial subcompartments. Based on these findings, water-soluble BS3 was used for further experiments. This avoided organic solvents that would have been necessary to resuspend water-insoluble DSS. Higher concentrations of organic solvents might disturb membrane protein interactions ${ }^{276}$, introducing artefacts. All identified crosslinks are listed in Supplementary Table 1 provided on a CD-ROM attached to the hardcopy version of this thesis.

DSS

$$
\begin{aligned}
& \text { - IM_IM } \\
& \text { - M_M } \\
& \text { - OM_OM } \\
& \text { - not mt } \\
& \text { - IM_M } \\
& \text { - IMS_IMS } \\
& \text { - ambig } \\
& \text { - OM_IM } \\
& \text { - IMS_M }
\end{aligned}
$$
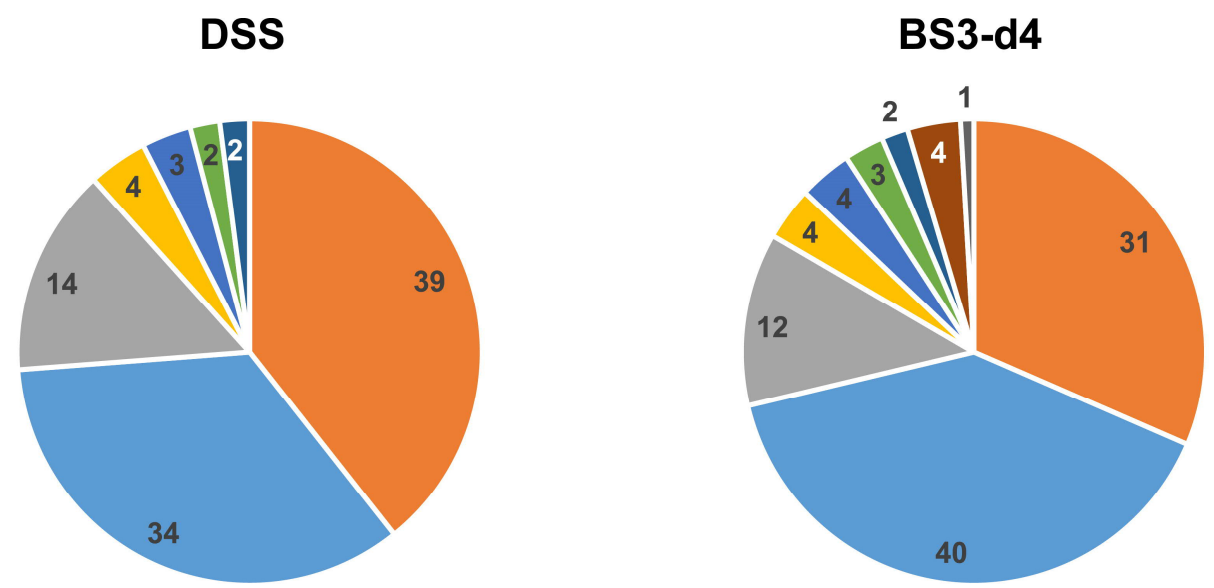

Figure 5: Comparison of both cross-linkers DSS and BS3 regarding their ability to cross-link proteins in all mitochondrial subcompartments. To investigate if DSS and BS3 are suitable for crosslinking of mitochondria derived from yeast, DSS and isotopically labeled BS3-d4 were mixed in an equimolar ratio and added to freshly isolated crude mitochondrial extract. The pie charts illustrate the relative number of unique protein-protein cross-links in percent depending on the suborganellar location of the proteins after quantitative cross-linking. Quantitation was performed by $\mathrm{XiQ}^{226}$ on identifications provided by pLink $1^{255,256}$. Only these protein-protein cross-links with quantified residue-to-residue cross-links showing a fold change $\geq 2$ were considered. OM, outer membrane; IMS, intermembrane space; IM, inner membrane; M, matrix; ambig, ambiguous; not mt, not mitochondrial. Adapted from Linden et al. ${ }^{247}$

\subsubsection{Workflow for cross-linking mitochondria}

Mitochondria were isolated from $S$. cerevisiae and purified via tandem sucrose gradient centrifugation ${ }^{239}$ (see subsections 2.2.3 and 2.2.4). Purified mitochondria of all conditions were cross-linked with BS3. Proteins were extracted and digested by the endoproteinase 
trypsin. Cross-linked peptides were enriched by peptide SEC and the first fractions containing the cross-linked peptides were subjected to LC-MS/MS. Scaffold ${ }^{253}$ was used to generate a protein database containing the 400 most abundant proteins based on the identification of linear peptides by Mascot $^{167}$ and pLink 1 was used for cross-linked peptides identification (Figure 6). Two biological replicates, i.e. cross-linking of different mitochondria isolations, were carried out per condition.

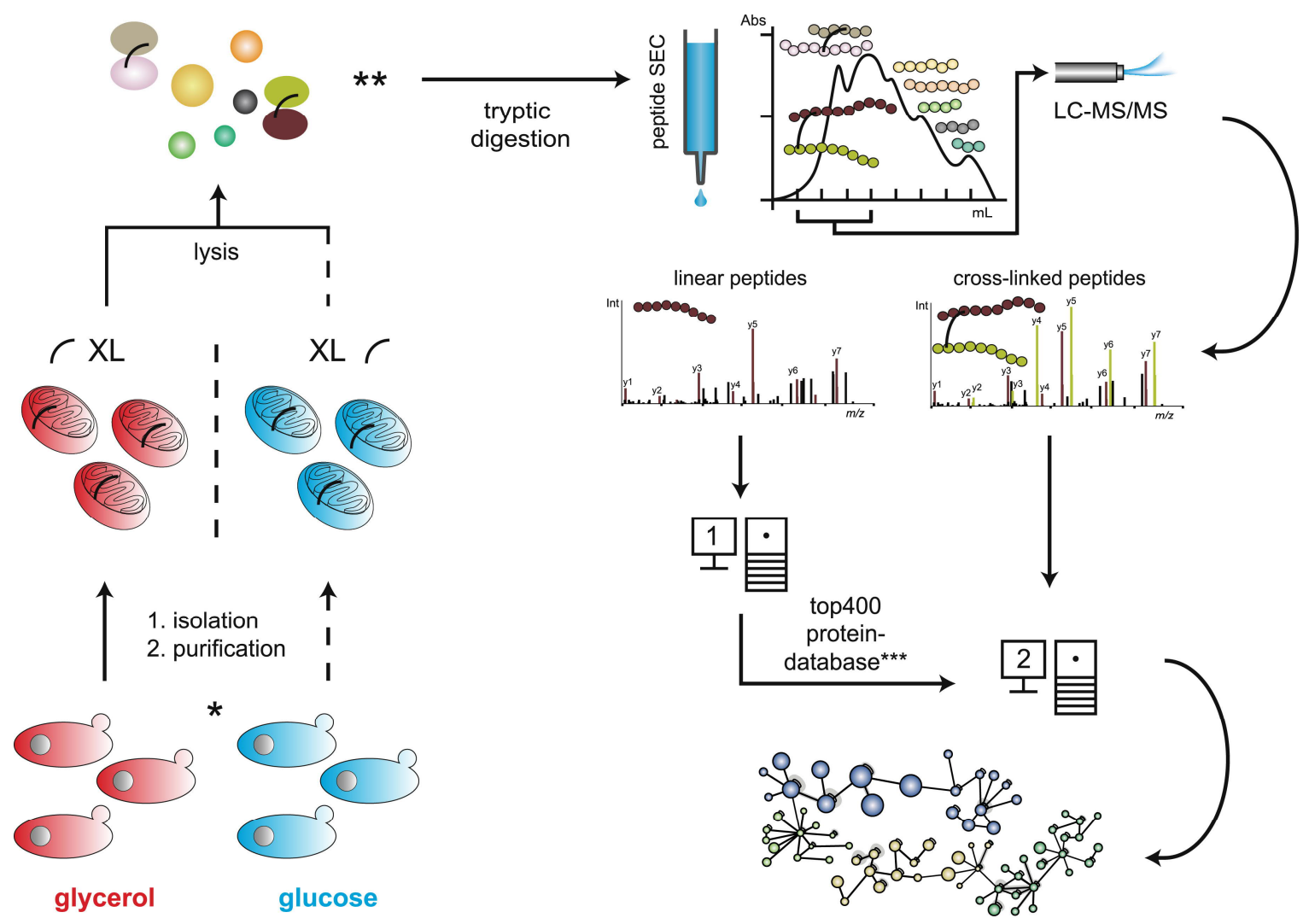

Figure 6: Cross-linking workflow for the identification of cross-linked peptides in mitochondria isolated from Saccharomyces cerevisiae. Yeast cells were grown on two different media individually, containing glycerol or glucose as carbon source. After isolation and purification via a sucrose gradient centrifugation, mitochondria were cross-linked with BS3 (XL; additional BS3-d4 was used for the quantitative experiments) and lysed. Proteins were tryptically digested and crosslinked peptides were enriched by peptide size exclusion chromatography. Linear peptides were identified by Mascot ${ }^{167}$ and Scaffold ${ }^{253}$ was used to generate a database containing the 400 most abundant proteins (computer "1"). Cross-linked peptides were identified by pLink 1255,256 (computer "2") by searching against the dedicated database. Depending on the experimental design, the workflow was adapted at steps marked with asterisks. ${ }^{*}$, a S. cerevisiae rho mutant was grown on glucose-containing medium and respective mitochondria were isolated; **, proteins of one biological replicate of mitochondria from yeast grown on glycerol-containing medium were first separated by protein size exclusion before digestion; ${ }^{* * *}$, for human mitoplasts, besides BS3 also EDC was used as $\mathrm{XL}$ and all identified proteins were included in the database, performed by MaxQuant ${ }^{254}$ on computer " 1 ", and analyzed with pLink $2^{258}$ on computer " 2 ". 
This resulted in 2999 unique residue-to-residue cross-links in the glycerol and 2595 unique residue-to-residue cross-links in the glucose data set ( $1 \%$ false discovery rate (FDR) on spectrum level), respectively. Next, those cross-linked peptides that were only identified by a single CSM or were below a pLink 1 spectrum score of four were excluded. This reduced the numbers of unique residue-to-residue cross-links to 2100 in the glycerol and 1787 in the glucose data set, with $17 \%$ (359) and $15 \%$ (266) of them being interprotein cross-links, i.e. cross-links between two different proteins. Cross-linking mitochondria from the $r h 0^{\circ}$ strain resulted in 1976 unique residue-to-residue cross-links (2922 before filtering), with $15 \%$ (298) interprotein cross-links (Table 6). With these stringent filtering steps, it was sought to reduce false positive cross-links. Due to the filtering steps, the overlap of unique residue-to-residue cross-links between biological replicates increased from $39 \%$ to $55 \%$ in the glycerol data set and from $45 \%$ to $62 \%$ in the glucose data set (Supplementary Figure 1). Cross-links were structurally validated by mapping identified cross-links from the glycerol data set onto available structures of proteins from different suborganellar locations (Supplementary Figure 2). $90 \%$ or above of these cross-links satisfied the distance constraint of $30 \AA$ imposed by the cross-linker BS3. All identified cross-links are listed in Supplementary Table 2 provided on a CD-ROM attached to the hardcopy version of this thesis.

Table 6: Numbers of total identified cross-linked peptides spectrum matches (CSMs), cross-linked proteins, unique residue-to-residue cross-links and the interprotein cross-links in percent for the unfiltered and the filtered data set for all conditions. 'Single CSMs' in percent represent those unique residue-to-residue cross-links that were identified only by a single CSM.

\begin{tabular}{|c|c|c|c|c|c|c|}
\hline & glycerol & $\begin{array}{l}\text { Infiltered } \\
\text { glucose }\end{array}$ & rhoo & glycerol & $\begin{array}{l}\text { filtered } \\
\text { glucose }\end{array}$ & rhoo \\
\hline total CSMs & 25022 & 22007 & 20538 & 23859 & 20508 & 19237 \\
\hline $\begin{array}{l}\text { cross-linked } \\
\text { proteins }\end{array}$ & 315 & 313 & 331 & 261 & 260 & 265 \\
\hline $\begin{array}{l}\text { unique residue- } \\
\text { to-residue cross- } \\
\text { links }\end{array}$ & 2999 & 2595 & 2922 & 2100 & 1787 & 1976 \\
\hline $\begin{array}{l}\text { interprotein } \\
\text { cross-links }\end{array}$ & $22.2 \%$ & $21.3 \%$ & $21.9 \%$ & $17.1 \%$ & $14.9 \%$ & $15.1 \%$ \\
\hline single CSMs & $29.7 \%$ & $30.5 \%$ & $32.0 \%$ & & & \\
\hline
\end{tabular}




\subsubsection{Cross-links reveal different protein-protein cross-linking patterns in yeast mitochondria depending on the carbon source}

To investigate whether the different carbon sources had a general effect on protein-protein cross-linking networks in the respective mitochondria, a global overview of the proteins that were involved in cross-linking reactions was generated. The filtered data sets revealed a total of 261 (glycerol) and 260 (glucose) proteins with an overlap of $66 \%$. They account for 396 and 386 unique protein-protein cross-links, respectively, subdivided into $42 \%$ (167) interprotein and $58 \%$ (229) intraprotein cross-links in the glycerol condition and $40 \%$ (155) interprotein and $60 \%$ (231) intraprotein cross-links in the glucose condition. First, the suborganellar localizations of these proteins were plotted according to Vögtle et al. ${ }^{142}$ (Figure 7). The majority of cross-links in both data sets covered protein-protein cross-links between IM proteins, followed by M-to-M protein-protein cross-links. Proteinprotein cross-links within the OM were less abundant. Cross-links that span one or even two intact membranes, e.g. OM-to-M, IMS-to-M or to non-mitochondrial proteins (not mt) are unlikely. These interactions account for less than $5 \%$ in the glycerol and the glucose data sets, underlining the reliability of these data.
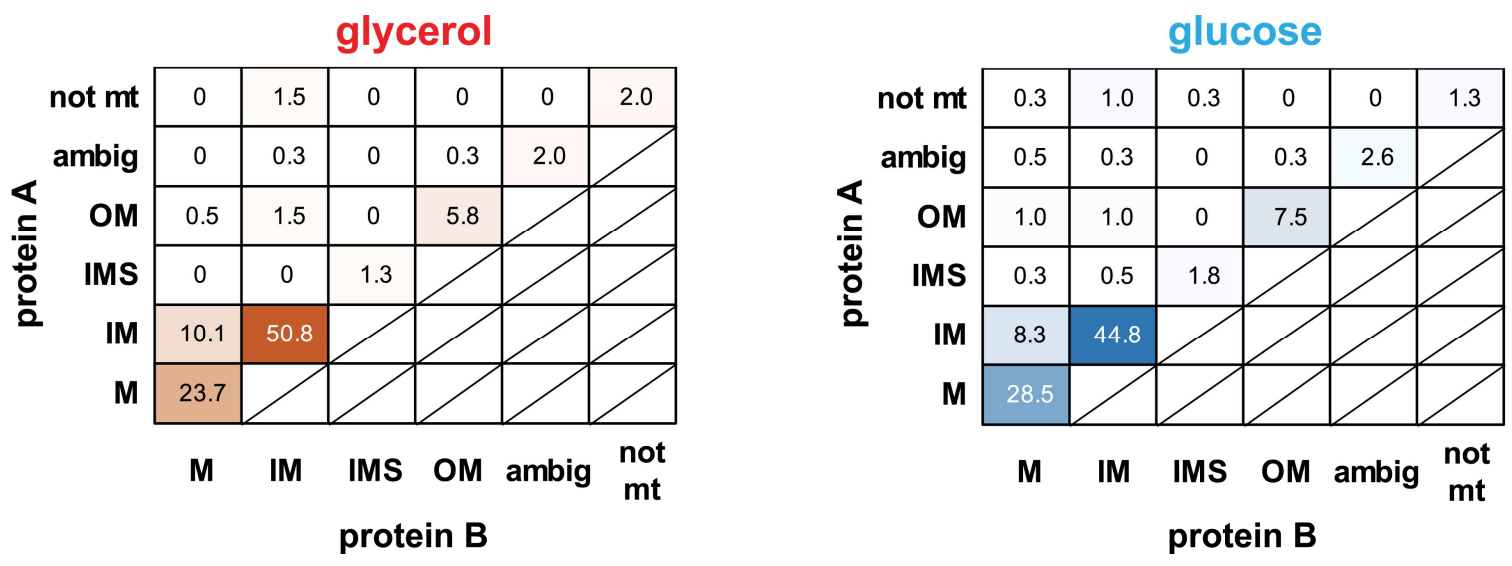

Figure 7: Relative distribution of protein-protein cross-links after cross-linking of mitochondria derived from yeast grown on glycerol- or glucose-containing medium according to the proteins' subcompartmental localizations. Intraprotein and interprotein cross-links were considered. Localization assignments are based on Vögtle et al. ${ }^{142} \mathrm{~A}$ Chi-square test of independence was conducted to calculate the significance of differences between protein-protein cross-links of both conditions with an assumed alpha level of $0.05\left(X^{2}=0.043\right)$. OM, outer membrane; IMS, intermembrane space; IM, inner membrane; $M$, matrix; ambig, ambiguous; not $\mathrm{mt}$, not mitochondrial. Adapted from Linden et al. ${ }^{247}$

When comparing data from the glycerol with the glucose condition, it was observed that IM-to-IM protein-protein cross-links were more abundant in the glycerol data set (201 (51\%) versus 173 (45\%) in the glycerol and the glucose data set, respectively), whereas 
3.1 Cross-linking combined with mass spectrometry of mitochondria derived from yeast grown on glycerol- or glucose-containing medium

M-to-M protein-protein cross-links were more abundant in the glucose data set (110 (29\%) versus $94(24 \%))$.
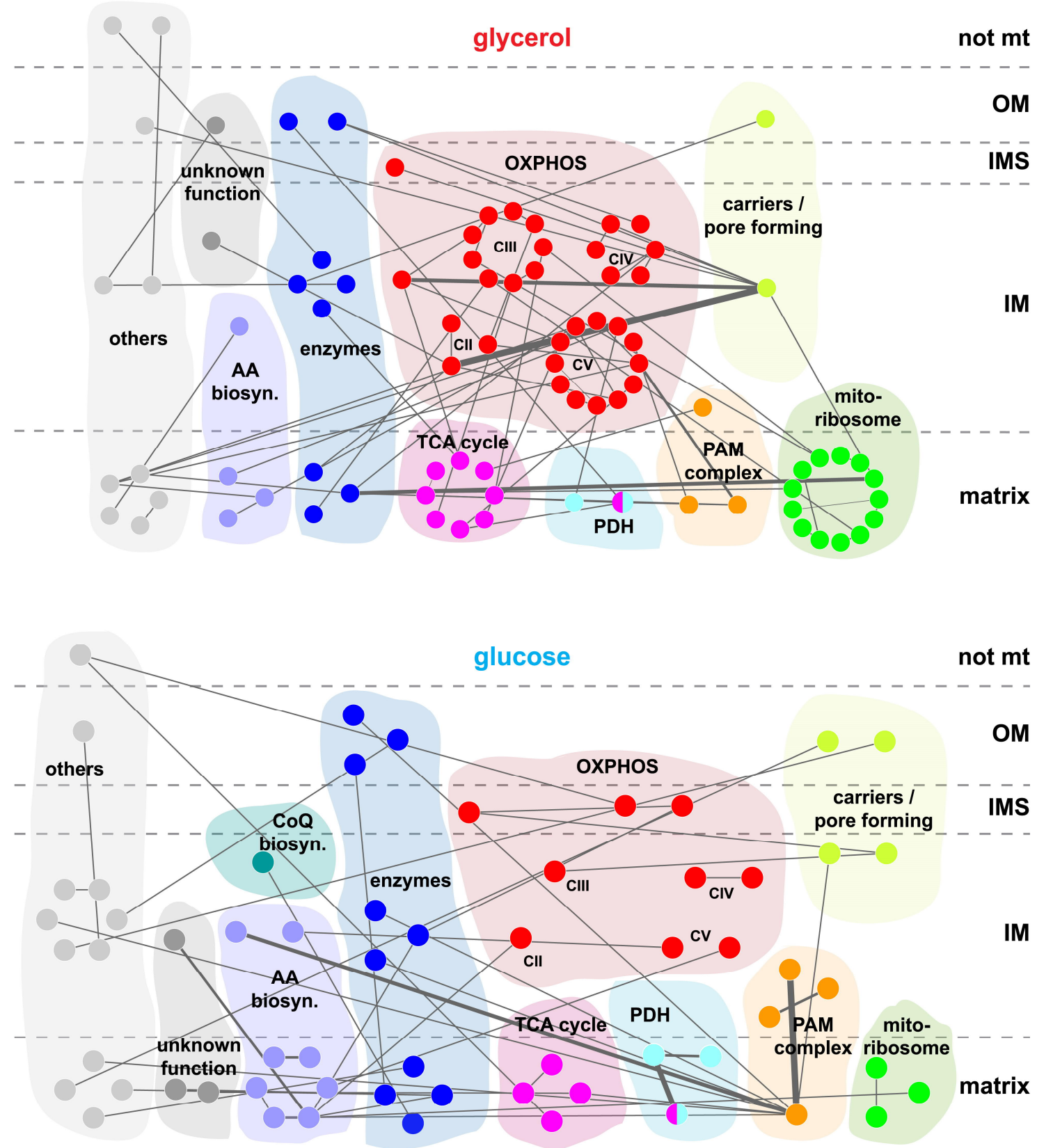

Figure 8: Global interprotein cross-linking network identified in mitochondria derived from yeast grown on glycerol- or glucose-containing medium. Interprotein cross-linking networks identified in the glycerol (upper panel) and the glucose data set (lower panel). Interprotein cross-links are unique for the respective condition, but all represented proteins were identified in both conditions. Localizations based on Vögtle et al. ${ }^{142}$ Thickness of the edges is proportional to the number of unique residue-to-residue cross-links. OM, outer membrane; IMS, intermembrane space; IM, inner membrane; ambig, ambiguous; not mt, not mitochondrial; AA biosyn., amino acid biosynthesis; CoQ biosyn, Coenzyme $Q$ biosynthesis; OXPHOS, oxidative phosphorylation system; TCA, tricarboxylic acid; PDH, pyruvate dehydrogenase complex; PAM, presequence translocaseassociated motor; mitoribosome, mitochondrial ribosome. Networks visualized by Cytoscape ${ }^{269}$. Adapted from Linden et al. ${ }^{247}$ 
In contrast to that, protein-protein cross-links of the OM were almost equally present (32 (8\%) versus $38(10 \%)$ ). Differences between protein-protein cross-links in both conditions are significant as evaluated by a Chi-square test of independence (see subsection 2.4.4).

Next, cross-linked proteins were assigned to known complexes. 74 unique interprotein cross-links within the electron transport chain (ETC), the tricarboxylic acid (TCA) cycle and the mitochondrial ribosome (mitoribosome) were common in both data sets (Supplementary Figure 3). Additionally, proteins of the presequence translocaseassociated import motor (PAM) complex, the pyruvate dehydrogenase complex (PDH), enzymes like transferases, isomerases, desulfurases and oxidases (e.g. Cpr3, Isd11, and Alo1) as well as carriers and pore forming proteins (e.g. Por1, Tom40, Pet9) were present in both conditions. Proteins that could not be sorted to any category were listed as 'others', e.g. prohibitin (Phb1, Phb2), DNA-binding proteins (e.g. Abf2) or translational activators (e.g. Mss51). Besides these commonalities, interprotein cross-links were also uniquely identified in each of the conditions. To map these proteins that were uniquely involved in interprotein cross-linking in either of the conditions to functional pathways, the KEGG database ${ }^{277}$ was used. Identifications from oxidative phosphorylation, metabolic and TCA cycle pathways were enriched in the glycerol data set, whereas 2-oxocarboxylic acid metabolism, biosynthesis of secondary metabolites as well as amino acids pathways were enriched in the glucose data set (data not shown). This is due to glucose-repression of genes that encode proteins involved in the OXPHOS system or the TCA cycle ${ }^{75,76}$. Consequently, more unique residue-to-residue cross-links within the ETC and the TCA cycle were identified in the glycerol than in the glucose data set (Figure 8, top panel). In the glucose data set, in turn, the number of unique residue-to-residue cross-links of proteins involved in amino acid biosynthesis (e.g. Ilv1-3, Ilv5, Lys4, Lys12) and coenzyme $\mathrm{Q}(\mathrm{CoQ})$ biosynthesis was higher (Figure 8, bottom panel).

\subsubsection{Cross-links between proteins localized to the inner membrane}

According to the relative distribution of cross-linked proteins (see subsection 3.1.3, Figure 7), the majority of protein-protein cross-links was identified between proteins localized to the IM. However, all mitochondrial subcompartments were covered with this cross-linking approach. To highlight this, cross-links of abundant proteins such as Por1 and Om45 (OM), Nde1 (IMS) and Pet9 (IM) were analyzed in more detail (Figure 9). Cross-links between Por1 and Om45, the most abundant outer membrane protein of so far unknown function ${ }^{278}$, were identified in both data sets. Their interaction was already described by Lauffer et al. ${ }^{279}$ Por1 is a voltage-dependent anion channel that is involved in ion transport and links the IMS to the cytosol. Cross-links of Por1 to Alo1, the D-arabinono-1,4-lactone 
oxidase, and to Tom40, component of the TOM complex, were also present in both data sets. The interaction between Por1 and Alo1 is uncharacterized so far, three unique residue-to-residue cross-links could be identified in the glycerol data set and five unique residue-to-residue cross-links in the glucose data set, respectively. Additionally, crosslinked peptides from Om45 and Nde1, the external NADH:ubiquinone oxidoreductase, could be detected. 22 unique residue-to-residue cross-links in the glycerol data set and eight residue-to-residue cross-links in the glucose data set indicate a strong interaction interface between Om45 and Nde1 that is also undescribed so far.

While these cross-links are common in both conditions, most of the changes in protein-protein cross-links were observed within the IM. Pet9, the major ADP/ATP carrier is one of the most abundant proteins in the $\mathrm{IM}^{97}$. Pet9 cross-linked to eleven proteins in the glycerol condition, but only to two proteins in the glucose condition (Figure 9). Among these eleven proteins that were identified in the glycerol condition, three of them belong to the ETC, namely Sdh2 (CII), Cor1 (CIII) and Cox9 (CIV), suggesting an association of Pet9 to the ETC. An interaction between Pet9 and Cor1 was also identified in the glucose data set. This finding is consistent with biochemical studies ${ }^{97,98}$. Pet9 also cross-linked to Ndi1 as well as Min8, an $8 \mathrm{kDa}$ protein of unknown function, that will be described in subsection 3.1.10.
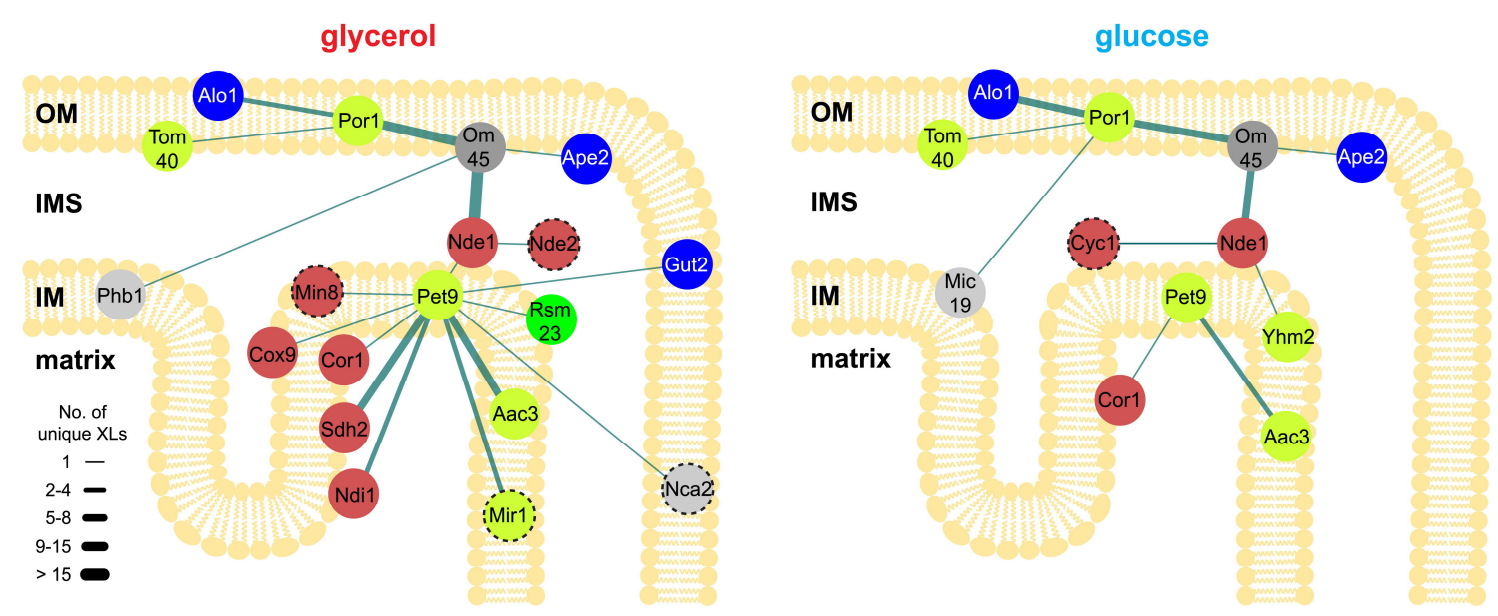

Figure 9: Cross-links of selected proteins as identified in mitochondria isolated from yeast grown on glycerol- or glucose-containing medium. Por1, Om45, Nde1 and Pet9 represent proteins from the OM, the IMS and the IM with a high number of unique residue-to-residue cross-links to interaction partners. These networks are visualized for the glycerol (left) and the glucose (right) condition. Proteins are colored as described in Figure 8 (red, ETC; blue, amino acid biosynthesis; yellow, carriers and pore forming proteins; green, mitoribosome; light gray, others; dark gray, unknown function). Dashed circles: database of the other condition did not cover these proteins. Thickness of the edges is proportional to the number of unique residue-to-residue cross-links. OM, outer membrane; IMS, intermembrane space, IM, inner membrane. Dr. Ralf Pflanz assisted in figure preparation. Adapted from Linden et al. ${ }^{247}$ 


\subsubsection{Cross-links within the OXPHOS system}

The main function of mitochondria is the generation of ATP. This is accomplished by the oxidative phosphorylation (OXPHOS) system consisting of four highly abundant multisubunit complexes in yeast (complexes II-V, CII-CV). CII-IV form the electron transport chain (ETC) generating an electrochemical gradient across the inner membrane by redox reactions and pumping protons from the matrix to the IMS. This proton gradient can finally be utilized by the $F_{1} F_{0}$ ATP synthase, also known as complex $V(C V)$, that synthesizes ATP from ADP and inorganic phosphate $\left(P_{i}\right)$. Yeast cells do not harbor complex I (Cl) but express Ndi1, a protein of $57 \mathrm{kDa}$. It functions as an $\mathrm{NADH}$ :ubiquinone oxidoreductase on matrix-side, but does not pump protons like mammalian $\mathrm{Cl}^{83}$. All these complexes were cross-linked in both conditions (Figure 10). However, more unique residue-to-residue cross-links were detected in the glycerol than in the glucose data set (383 unique residue-to-residue cross-links in the glycerol data set versus 191 residue-toresidue cross-links in the glucose data set). Also, CIV-associated proteins such as Rcf2 and Rcf3 that facilitate the complex assembly were only identified in the glycerol data set, in addition to Min8. These proteins and their cross-links will be discussed below (see subsections 3.1.8 and 3.1.10). These results indicate a densely packed ETC, with Ndi1 (see subsection 3.1.9) and Pet9 being part of it.
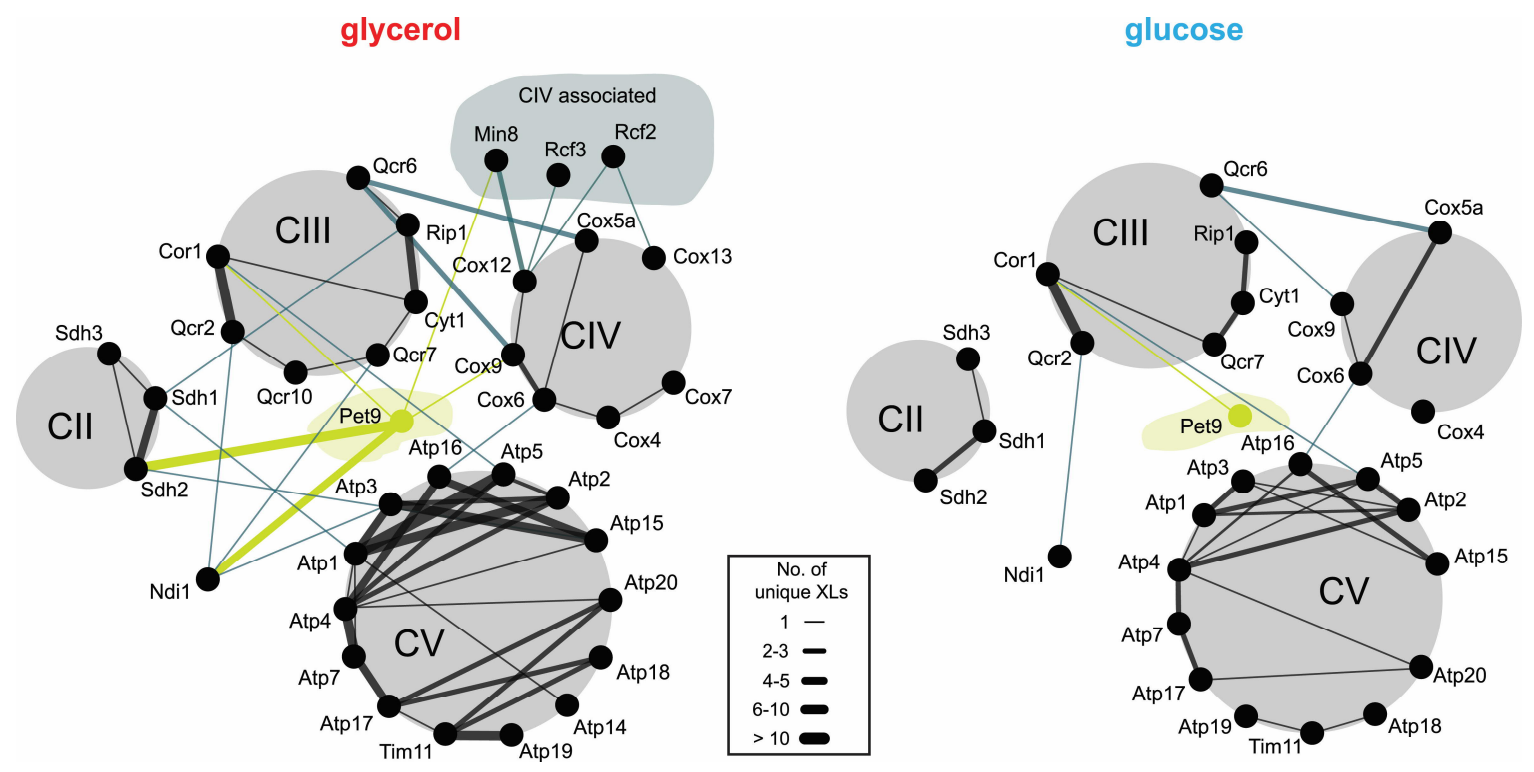

Figure 10: Interprotein cross-links within the OXPHOS system identified in mitochondria derived from yeast grown either under glycerol (left) or glucose (right) condition. Ndi1, $\mathrm{F}_{1} \mathrm{~F}_{0}$ ATP synthase (CV), CIV-associated proteins and Pet9 (yellow) are included. Thickness of the edges is proportional to the number of unique residue-to-residue cross-links. Black lines, intra-complex cross-links; turquoise lines, inter(-complex) cross-links. Dr. Ralf Pflanz assisted in figure preparation. Adapted from Linden et al. ${ }^{247}$ 
3.1 Cross-linking combined with mass spectrometry of mitochondria derived from yeast grown on glycerol- or glucose-containing medium

\subsubsection{Quantitative XL-MS approach reveals abundance changes of residue- to-residue cross-links depending on the carbon source}

To further elucidate the differences in residue-to-residue cross-links within mitochondria from yeast grown on glycerol- or glucose-containing medium and to quantify them, a labelswap experiment with isotopically labeled BS3 was performed. For that, mitochondria

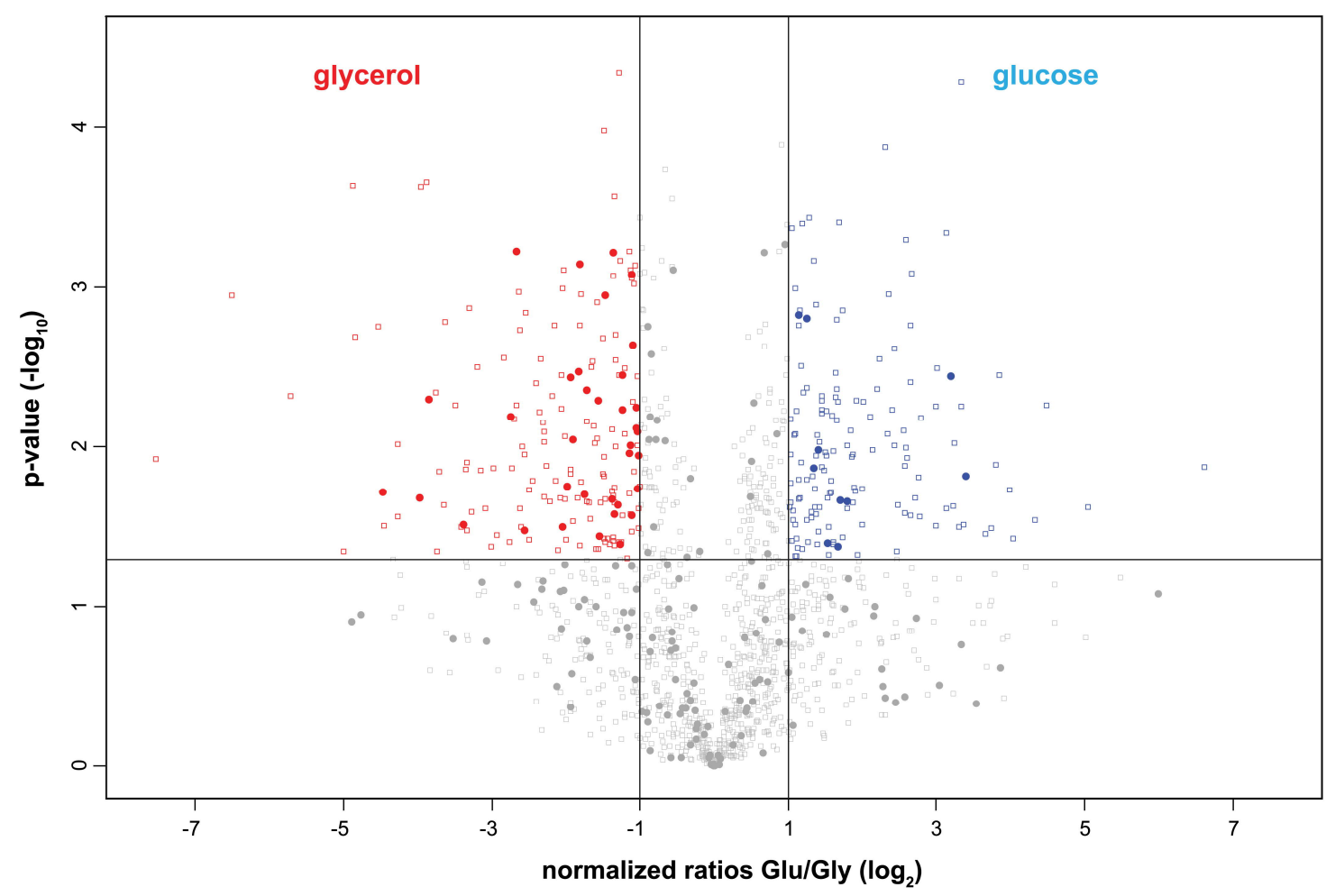

Figure 11: Results from quantitation of unique residue-to-residue cross-links after cross-linking mitochondria derived from yeast grown on glycerol- or glucose-containing medium with isotopically labeled and unlabeled BS3. Volcano plot of $p$ value, - $\log _{10}$-transformed, against normalized heavy (glucose, blue) to light (glycerol, red) ratios, $\log _{2}$-transformed. Thresholds were set at $p$ value $\leq 0.05$ and fold change $\geq 2$. Filled circles, interprotein cross-links; squares, intraprotein cross-links. Adapted from Linden et al. .47 $^{2}$

isolated from yeast grown on glycerol-containing medium were cross-linked with isotopically labeled BS3-d4 whereas mitochondria from yeast grown on glucosecontaining medium were incubated with non-labeled BS3-d0 and vice versa (see subsection 2.2.13). Samples were mixed in a 1-to-1 ratio according to the protein amount. The sample preparation was performed as described in subsection 3.1.2. Cross-linked peptides identification was performed by pLink 1 against a database containing the 400 most abundant proteins based on identification of linear peptides with MaxQuant ${ }^{254}$ (see subsection 2.4.1). The quantitation of cross-linked peptides on MS1 level was performed by $\mathrm{XiQ}^{226}$ (see subsection 2.4.1.3). A fold change $\geq 2$ and a $p$ value $\leq 0.05$ with three valid 
values out of four replicates was considered as significant (see subsection 2.4.4). 169 unique residue-to-residue cross-links (accounting for 46 proteins) had significantly higher MS1 intensities in the glycerol condition. In the glucose condition, 145 unique residue-toresidue cross-links (62 proteins) had significantly higher MS1 intensities (Figure 11). Of these 169 residue-to-residue cross-links in the glycerol condition, 134 (75\%) represents intraprotein cross-links, 135 of 145 (93\%) in the glucose condition. All identified crosslinks are listed in Supplementary Table 1 provided on a CD-ROM attached to the hardcopy version of this thesis.

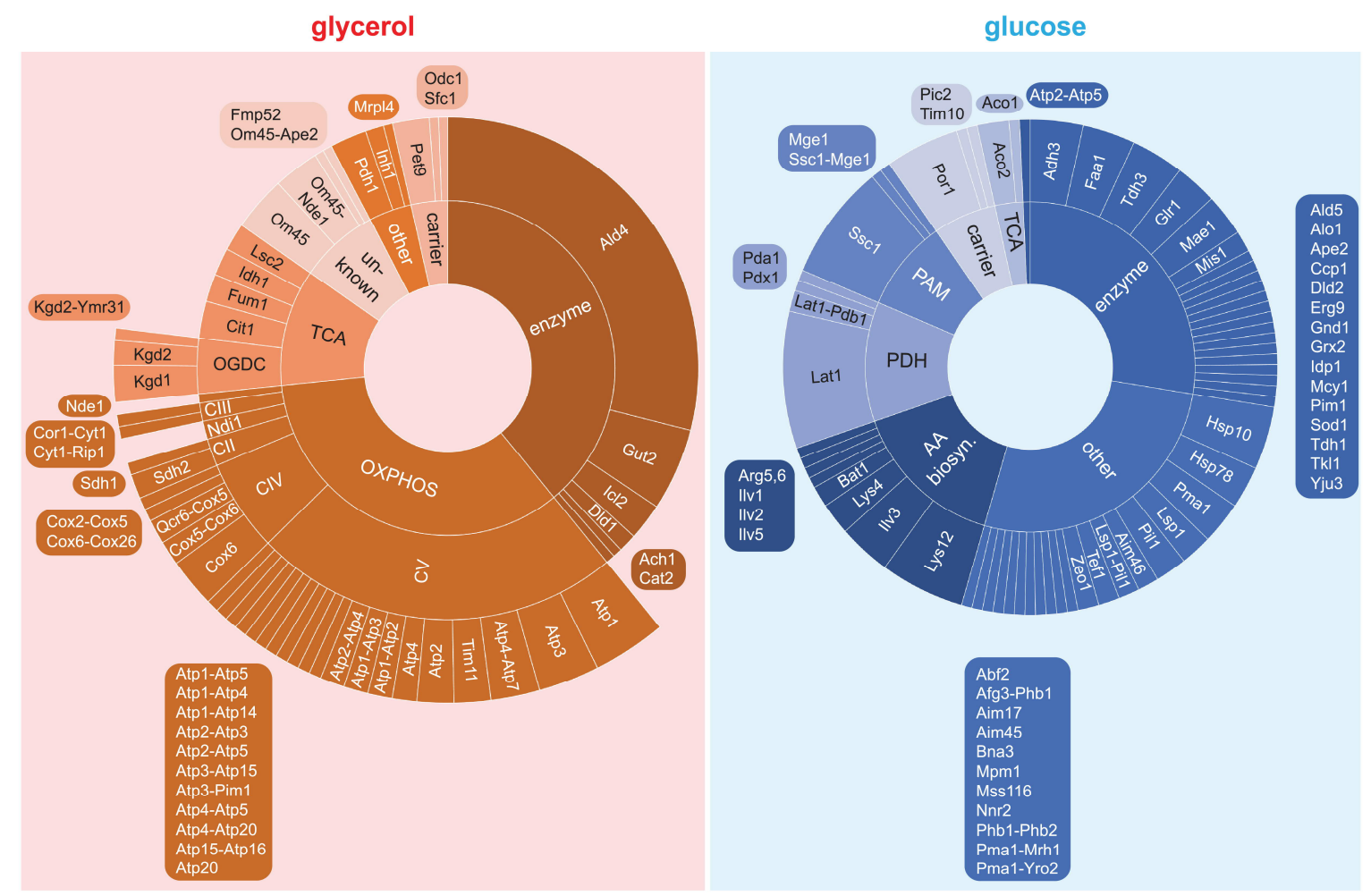

Figure 12: Representation of intraprotein and interprotein cross-links that were quantified after cross-linking mitochondria derived from yeast grown on glycerol- or glucose-containing medium with isotopically labeled and unlabeled BS3. Protein-protein cross-links were clustered according to the function of involved proteins (similar to Figure 8), depicted as sunburst diagrams. Area of a field is proportional to the number of unique residue-to-residue cross-links. Red, depicting crosslinks with higher intensities in glycerol growth condition; blue, depicting cross-links with higher intensities in glucose growth condition. AA biosyn., amino acid biosynthesis; OXPHOS, oxidative phosphorylation system; TCA, tricarboxylic acid; PDH, pyruvate dehydrogenase complex; PAM, presequence translocase-associated motor. Adapted from Linden et al. ${ }^{247}$

Similar to the qualitative data set, a KEGG ${ }^{277}$ pathway enrichment analysis of the 46 and 62 respective proteins was performed. Also in this quantitative data set, oxidative phosphorylation, metabolic and TCA cycle pathways were enriched in the glycerol condition. 2-oxocarboxylic acid metabolism, biosynthesis of secondary metabolites as well 
as amino acids pathways were enriched in the glucose condition (data not shown), underlining the effect of glucose-repression ${ }^{75,76}$. In the amino acid biosynthesis pathway especially cross-links of Ilv2, Ilv3, Ilv5, Lys4 and Lys12 showed higher intensities. Furthermore, intra- and interprotein cross-links within parts of the PAM complex, namely Ssc1 and Mge1, were more abundant in the glucose condition. Also, intraprotein crosslinks of the outer membrane transporter Por1 were more abundant (Figure 12, right diagram).

In the glycerol condition, the protein with the highest number of unique residue-toresidue cross-links that were significantly more abundant was Ald4, followed by intracomplex links of CV. However, also a higher intensity of the cross-links between Qcr6 of CIII and Cox5a of CIV, subunits that are in close contact within an ETC supercomplex (see subsection 3.1.9), could be observed. Moreover, the quantitative data confirmed that the cross-links between Om45 and Nde1 are more abundant in the glycerol condition. Additionally, a cross-link between Cox6 and Cox26, a newly identified subunit of $\mathrm{CIV}^{124,125}$, was more abundant in the glycerol data set (Figure 12, left diagram).

To summarize, the quantitative data fit well to the qualitative data. However, most of the quantified cross-linked proteins, especially in the glycerol data set, were found with a higher copy number per se, depending on the growth condition. This is consistent with the data from Morgenstern et al. ${ }^{72}$ (Figure 13) and with the performed proteomic analysis in this thesis (see section 3.4). In this regard, increased intensities of all intraprotein crosslinks could be due to the higher copy numbers of the proteins induced by the respective carbon source. However, differences in abundances of interprotein cross-links can provide valuable insights into interaction dynamics, especially between proteins or subunits of complexes that show comparable copy numbers in both growth conditions. One example for this is the pyruvate dehydrogenase complex (PDH).

\section{glycerol}

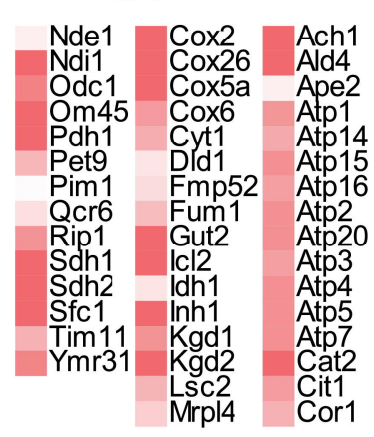

\section{glucose}

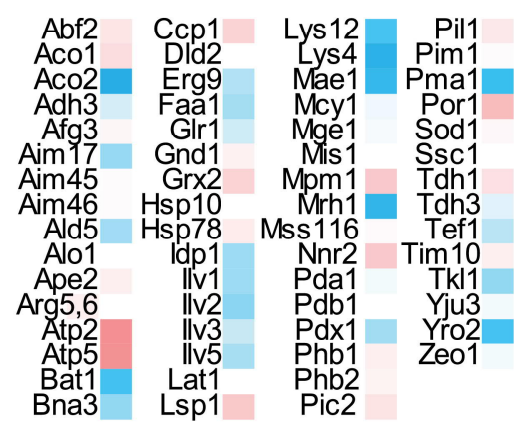

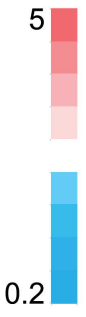

ratio copy numbers glycerol/glucose*

Figure 13: Ratios of copy numbers of quantified cross-linked proteins in S. cerevisiae grown under glycerol (left) and glucose (right) condition. Red, higher copy number in glycerol; blue, higher copy number in glucose. *, values taken from Morgenstern et al. ${ }^{72}$ Adapted from Linden et al. ${ }^{247}$ 


\subsubsection{Organization of the pyruvate dehydrogenase complex}

To evaluate structural changes between proteins protein complexes induced by the different carbon sources, cross-links between proteins that showed an equal copy number in both the glycerol and the glucose condition were investigated (according to Morgenstern et al. $^{72}$ ). One example for this is the pyruvate dehydrogenase complex (PDH), a multienzyme machine in the matrix of mitochondria. It synthesizes acetyl-CoA from the substrate pyruvate, and, thus, links the glycolysis to the TCA cycle. E1, the pyruvate dehydrogenase, is composed of two subunits, Pda1 and Pdb1, and surrounds E2, the dihydrolipoyl transacetylase Lat1 together with E3, the dihydrolipoyl dehydrogenase Lpd1. The assembly of E2 and E3 is facilitated by the E3-binding protein Pdx1, forming a fully functional $\mathrm{PDH}^{280}$. While Lpd1 showed a 2.4-fold higher copy number in the glycerol condition and Pdx1 a 1.4-fold higher copy number in the glucose condition, Pda1, Pdb1 as well as Lat1 had equal copy numbers in both conditions. 13 unique intraprotein crosslinks of Lat1, one intraprotein cross-link of each of the proteins Pda1 and Pdx1 and two interprotein cross-links between Lat1 and Pdb1 could be quantified. All of them were significantly more abundant in the glucose condition. These quantitative data show that the intensities of cross-linked peptides are not only related to the copy numbers of the respective proteins. Differences in the abundance of cross-links can also reflect structural arrangements of protein complexes depending on the carbon source.

These quantitative results were then compared to the respective cross-links in the qualitative data sets. Here, the number of unique residue-to-residue cross-links within the $\mathrm{PDH}$ was also higher in the glucose condition (Figure 14). In the glucose data set, 107 residue-to-residue cross-links were identified versus 83 residue-to-residue cross-links in the glycerol data set. Of these, 40 residue-to-residue cross-links were uniquely identified in the glucose condition and 14 residue-to-residue cross-links in the glycerol condition. Strikingly, the E3-binding protein $\mathrm{Pdx} 1$ formed two unique residue-to-residue cross-links to each of E2 and E3 in the glucose data set. In contrast to that, only one unique residueto-residue cross-link was detected between $\mathrm{Pdx} 1$ and E3 in the glycerol data set. This correlates with an increase in interprotein cross-links between E2 and E3 (four unique residue-to-residue links in the glucose data set versus none in the glycerol data set). The identified cross-links might support a more detailed structural evaluation of the PDH whose structure still needs to be unraveled in yeast. 


\section{glycerol versus glucose}

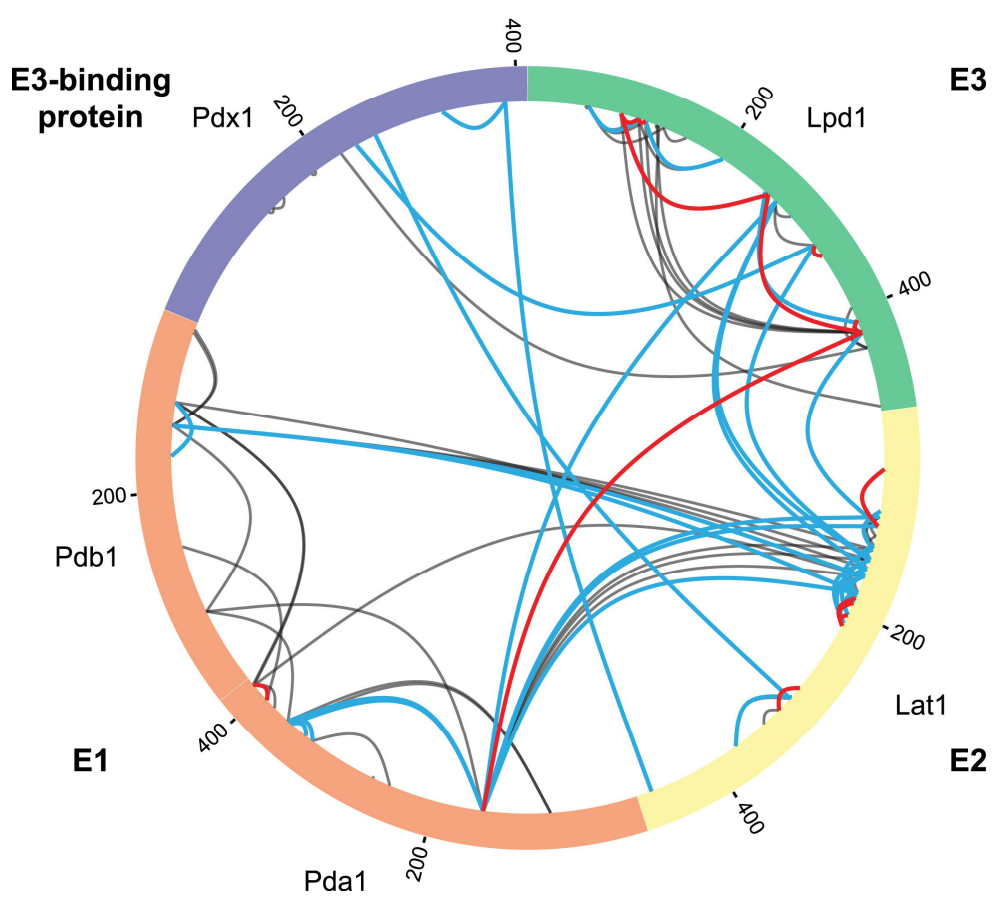

Figure 14: Cross-links within the pyruvate dehydrogenase complex (PDH) identified in mitochondria derived from yeast grown on glycerol- or glucose-containing medium. Intraprotein and interprotein cross-links within PDH from the qualitative data sets. Blue lines, cross-links only identified in glucose condition; red lines, cross-links only identified in glycerol condition; black lines, common links. Visualized by $x \mathrm{Vis}^{271}$. Adapted from Linden et al. ${ }^{247}$

\subsubsection{Interactions of uncharacterized proteins}

According to the Saccharomyces Genome Database ${ }^{29}$ (SGD), 726 out of 6,604 open reading frames (ORFs) are still uncharacterized (April 2020). Characterization of the resulting proteins will help to localize them and to describe their function. So far uncharacterized proteins in both qualitative data sets were investigated. To prove data validity, proteins that have been described recently but whose function is still under discussion were also included. In the glycerol condition, ten of these proteins were identified. Four of them, namely Min8, Nat2, Rcf3 and Ycp4 showed interprotein crosslinks. In the glucose data set, eleven proteins were identified. Aim17, Coq21, Dpi8, Fmp16 and the protein encoded by the ORF YDR061W provided interprotein cross-links (Supplementary Figure 5). Aim17 cross-linked to cyclophilin Cpr3 (one unique residueto-residue cross-link), and Fmp16 to Aco1, the aconitase within the TCA cycle (one unique residue-to-residue cross-link). Coq21 cross-linked to Coq5 (one unique residue-to-residue cross-link). This is consistent with the study performed by Morgenstern et al. ${ }^{72}$. The authors could localize Coq21 to the matrix and by immunoprecipitation define it as part of the coenzyme $Q$ biosynthesis cluster. Dpi8, also localized to the matrix in the same study but 
not further characterized, cross-linked to Kgd1 (one unique residue-to-residue cross-link). $\mathrm{Kgd1}$ is part of the $\alpha$-ketoglutarate dehydrogenase of the TCA cycle. The ORF YDR061W is uncharacterized so far. The protein encoded by this ORF shows similarity to ABC transporter. In the glucose data set, two unique residue-to-residue cross-links to Ilv5, an acetohydroxyacid reductoisomerase involved in amino acid biosynthesis could be identified.

In the glycerol data set, Nat2 cross-linked to Mdh1, the malate dehydrogenase within the TCA cycle (one unique residue-to-residue cross-link). Ycp4, a flavodoxin-like protein, showed one unique residue-to-residue cross-link to Hsp60 and Pst2 (like Ycp4 a protein with similarity to flavodoxin-like proteins), respectively. Furthermore, Rcf3 crosslinked to Cox12, a subunit of CIV (one unique residue-to-residue cross-link). Rcf proteins are respiratory supercomplex factors and interact with CIV. They assist in the assembly process of the CIII-CIV supercomplex. This is consistent with the study performed by Römpler et al. ${ }^{119}$ who identified Rcf3 as homolog of Rcf2 and their association with CIII and CIV. Also, Rcf2 could be identified in the glycerol data set, showing one unique residue-to-residue cross-link to each of Cox12 and Cox13. While the N-terminus of Rcf3 cross-linked to the N-terminus of Cox12, Rcf2 cross-linked C-terminally with both Cox12 and Cox13. Hence, all identified cross-links occurred in the IMS that is in accordance with the proposed inner membranous localization and orientation of both Rcf proteins. Römpler et al. ${ }^{119}$ demonstrated that Rcf2 is processed after import into a stable C-terminal part of Rcf2 and an unstable N-terminal part. The identified cross-links confirm the association of Rcf2 and Rcf3 with CIV and their role in late stage assembly of Cox12 and Cox13. Furthermore, Min8 cross-linked to Cox12 as well and additionally cross-linked to Pet9.

\subsubsection{Ndi1 is part of an ETC supercomplex}

Previous studies have shown that CIII and CIV form functional supercomplexes in eukaryotes ${ }^{87,88}$. It is hypothesized that formation of supercomplexes support faster electron shuttling ${ }^{103}$. With most of the biochemical methods that were used to study the CIII-CIV supercomplex, native conditions were altered due to the presence of detergents. XL-MS is able to catch interactions in organello and was already applied to murine mitochondria to prove supercomplex formation ${ }^{99,100}$. In order to confirm supercomplex formation also in yeast, the cross-links within the ETC were analyzed in more detail. Since the number of identified cross-links within the ETC was higher in the glycerol condition, only cross-links identified in mitochondria from yeast grown on glycerol-containing medium were considered for further analyses (see subsection 3.1.5, Figure 10, left panel). Genes that encode proteins involved in OXPHOS are glucose-repressed ${ }^{75,76}$. While the majority of the 
3.1 Cross-linking combined with mass spectrometry of mitochondria derived from yeast grown on glycerol- or glucose-containing medium

cross-links represents intra-complex cross-links, i.e. cross-links within or between subunits of the same ETC complex, 13 unique inter-complex cross-links were identified. Five of those interprotein cross-links were identified between Qcr6, a subunit of CIII, and Cox5a as well as Cox9, both subunits of CIV. In a CIII-CIV supercomplex, the subunits Qcr6 and Cox $5 \mathrm{a}$ are in closest proximity at the interface ${ }^{93}$, which yielded the highest number of unique residue-to-residue cross-links (three). $90 \%$ of these residue-to-residue cross-links matched to the recently published $\mathrm{CIII}_{2} \mathrm{CIV}_{2}$ supercomplex structure ${ }^{91}$ (data not shown). This indicates that CIII and CIV are in close proximity to each other and form a supercomplex structure in organello.

In mammals, $\mathrm{Cl}$ is part of a $\mathrm{Cl}$-CIII-CIV supercomplex ${ }^{87}$. Since $\mathrm{Cl}$ is absent in yeast, it was further investigated whether Ndi1 might take over this part. This hypothesis is supported by two unique residue-to-residue cross-links to CIII, one between residues K76 of Ndi1 and K17 of Qcr7, the other one between residues K361 of Ndi1 and K198 of Qcr2. Based on this cross-links, the theoretical interaction space between Ndi1's dimeric structure ${ }^{263}$ and the $\mathrm{CIII}_{2} \mathrm{CIV}_{2}$ supercomplex structure ${ }^{91}$ was calculated by DisVis ${ }^{273}$. It demonstrates that there is no steric hindrance to CIV (Figure 15). Thus, the data are compatible with a participation of Ndi1 in an ETC supercomplex in yeast.
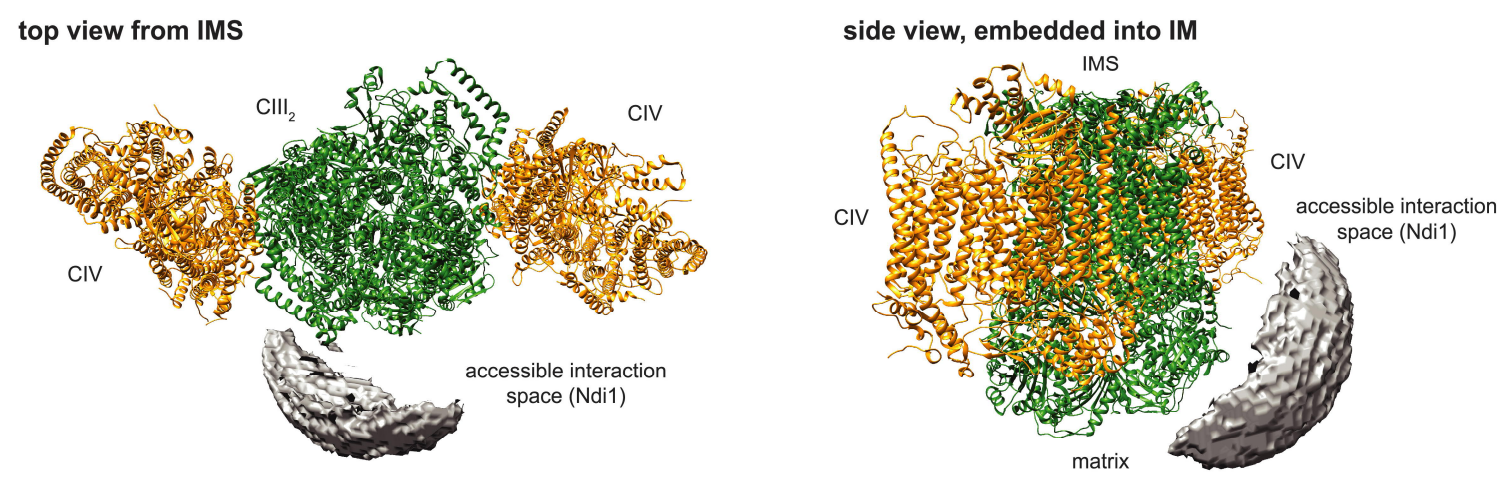

Figure 15: $\mathrm{CIII}_{2} \mathrm{CIV}_{2}$ supercomplex structure with calculated accessible interaction space of Ndi1. Accessible interaction space of Ndi1 (gray sphere) within a $\mathrm{Ndi}_{1} \mathrm{Cll}_{2} \mathrm{CIV}_{2}$ supercomplex based on identified cross-links in mitochondria derived from yeast grown on glycerol-containing medium. Visualized as top view from IMS (left) and as side view embedded into IM (right). Accessible interaction space calculated by DisVis 273 . IMS, intermembrane space; IM, inner membrane. Structures and spheres were illustrated by UCSF Chimera ${ }^{274}$. Adapted from Linden et al. ${ }^{247}$

Next, a structural arrangement for an $\mathrm{Ndi1}_{1} \mathrm{ClI}_{2} \mathrm{CIV}_{2}$ supercomplex was proposed. Therefore, Rosetta ${ }^{259-262}$ was used to dock the structure of dimeric $\mathrm{Ndi1}^{263}$ to the recently published $\mathrm{CIII}_{2} \mathrm{CIV}_{2}$ supercomplex structure ${ }^{91}$ based on the identified cross-links and Ndi1's C-terminal membrane anchor (Figure 16, see subsection 2.4.3). As confirmed by the DisVis analysis, there is no steric hindrance to CIV. Ndi1 forms a T-like shape with the 
CIII-CIV supercomplex as seen from the IMS. This shape is comparable to the mammalian $\mathrm{CICIII}_{2} \mathrm{CIV}$ supercomplex (Supplementary Figure 4). An $\mathrm{Ndi}_{2} \mathrm{CII}_{2} \mathrm{CIV}_{2}$ supercomplex could also be constructed by docking Ndi1 on both sides of the dimeric CIII, forming a propeller-like shape with Ndi1-CIII $-\mathrm{Ndi1}$ on the one axis and CIV-CIII ${ }_{2}$ CIV on the other axis. The data provide evidence for a participation of $\mathrm{Ndi1}$ in an $\mathrm{Ndi1}_{1} \mathrm{CII}_{2} \mathrm{CIV}_{2}$ or even higher-order supercomplex arrangement.

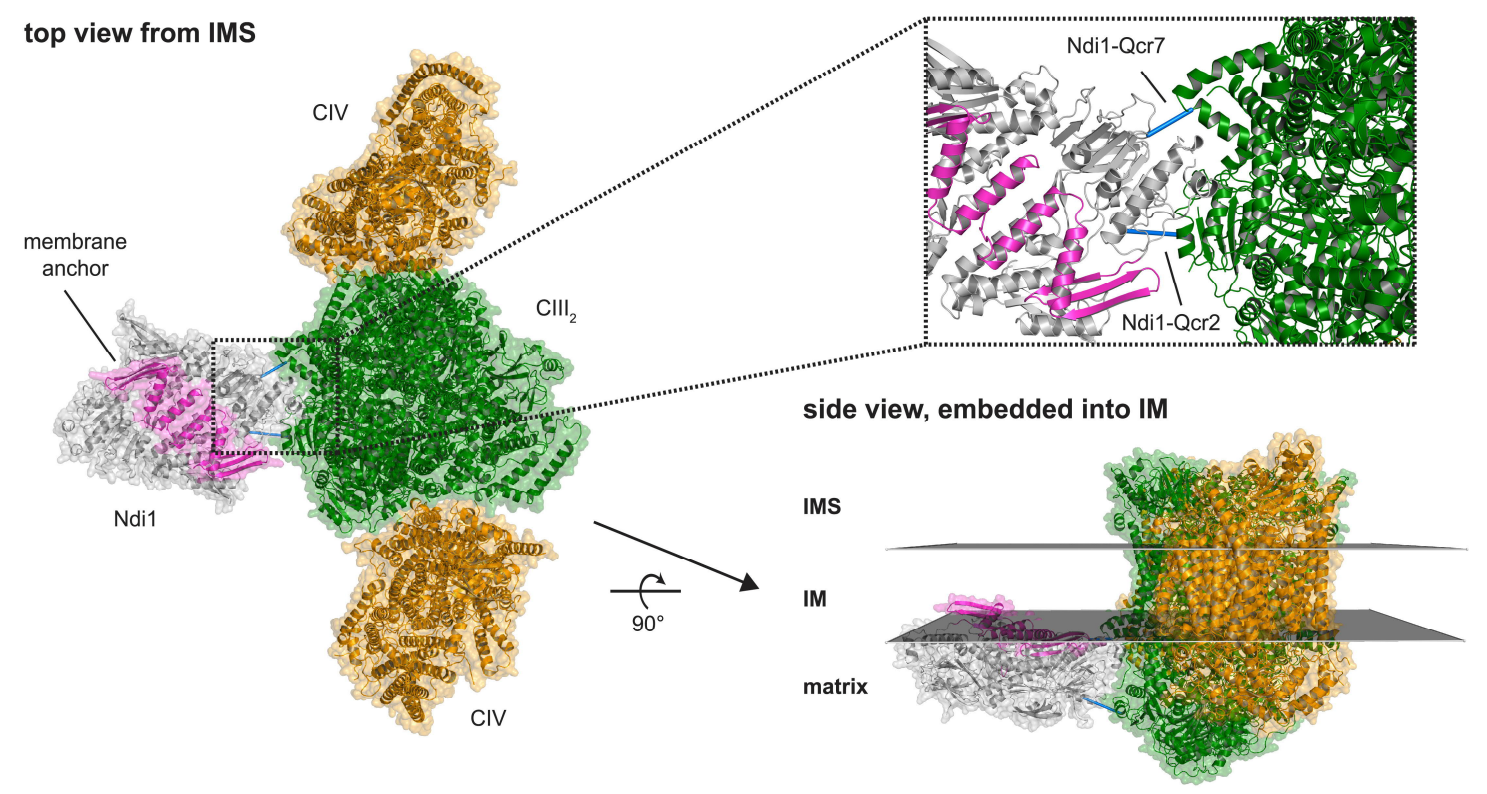

Figure 16: Proposed structure of an $\mathrm{Ndi}_{1} \mathrm{ClI}_{2} \mathrm{CIV}_{2}$ supercomplex in yeast. Representation of the supercomplex as top view from IMS (left) and as side view embedded into IM (bottom right). Ndi1 dimer (gray; light purple, membrane anchor; PDB: 4G73263) was docked to the $\mathrm{Clll}_{2} \mathrm{CIV}_{2}$ supercomplex structure (green, Clll dimer; orange, CIV monomers; PDB: 6HU991) based on the identified cross-links between Ndi1 and CIII in mitochondria derived from yeast grown on glycerolcontaining medium. Inlet, zoomed interaction interface between CIII and Ndi1. IMS, intermembrane space; IM, inner membrane. Docking experiments were performed by Dr. Piotr Neumann. Adapted from Linden et al.247

\subsubsection{Min8 is associated with the cytochrome $c$ oxidase}

Min8 is a small protein consisting of 72 amino acids with an uncharacterized open reading frame (ORF) YPR010C-A and cross-linked to CIV similar to the Rcf proteins. Its C-terminus showed two unique residue-to-residue cross-links to Cox12, a subunit of CIV. Additionally, Min8 cross-linked to Pet9 with its N-terminus. Pet9 is the major ADP/ATP carrier protein within the IM whose association with the ETC was discussed above. Min8 was already assigned as a protein of the IM by Morgenstern et al. ${ }^{72}$, with its $\mathrm{C}$-terminus protruding to the IMS, its $\mathrm{N}$-terminus protruding to the matrix and a transmembrane region between amino acid positions 20 and 40 that is embedded into the inner membrane. Rosetta ${ }^{265-268}$ 
3.1 Cross-linking combined with mass spectrometry of mitochondria derived from yeast grown on glycerol- or glucose-containing medium

was used to calculate a model of Min8 ab initio. Next, the modeled Min8 structure was docked to CIV whose structure was extracted from the $\mathrm{CIII}_{2} \mathrm{CIV}_{2}$ supercomplex structure ${ }^{91}$ (see subsection 2.4.3). The structure of Pet9 and its cross-links to Min8 and to CIV were included in the docking experiment (Figure 17). Reactive lysine residues of Min8 were K69 on IMS and K13 on matrix-side. K69 linked to K41 as well as the N-terminus of Cox12. $\mathrm{K} 13$ cross-linked to K182 of Pet9 on the matrix-side. The model and the cross-links to CIV and Pet9 indicate an association of Min8 to CIV.
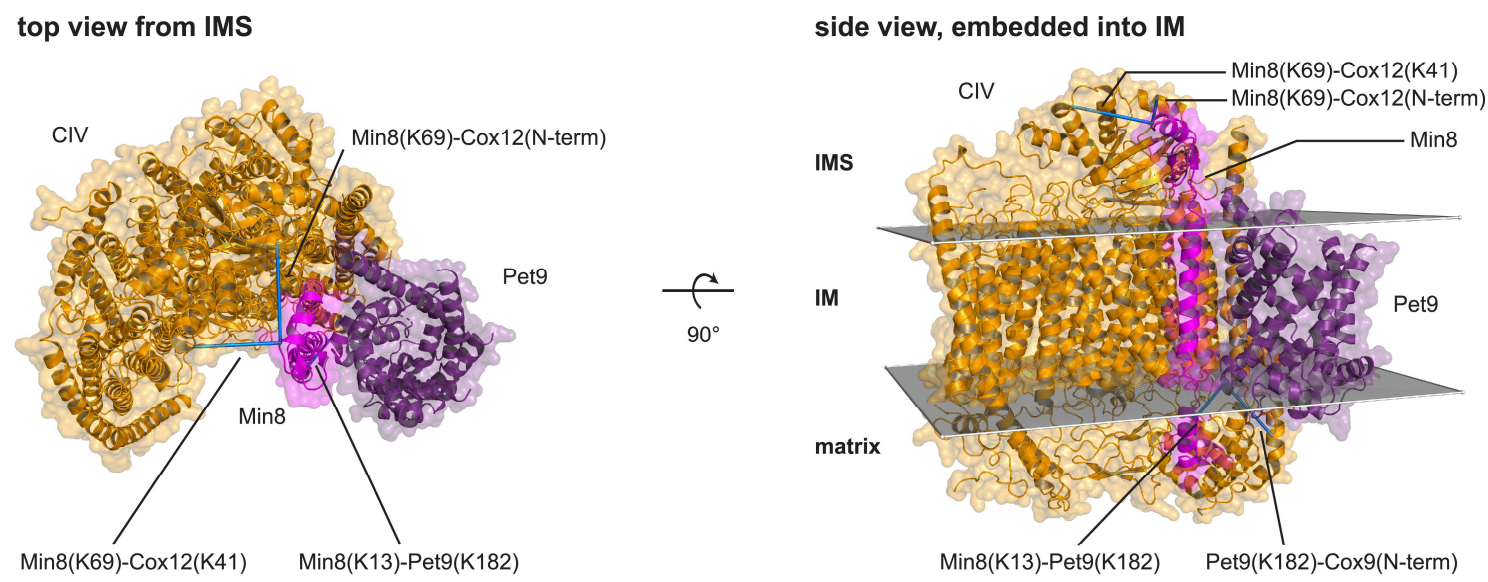

Figure 17: Proposed structural model of Min8 associated with the cytochrome $c$ oxidase. Min8 (magenta) was modeled $a b$ initio and docked to the cytochrome $c$ oxidase (extracted from PDB: 6HU991) together with Pet9 (purple; PDB: 4C9G ${ }^{264}$ ) based on cross-links identified in mitochondria derived from yeast grown on glycerol-containing medium. Demonstrated are the top view from IMS (left) and the side view embedded into IM (right). Identified residue-to-residue cross-links are represented as blue bars, satisfying the distance constraint of $30 \AA$ given by the cross-linker BS3. IMS, intermembrane space; IM, inner membrane. Docking experiments were performed by Dr. Piotr Neumann. Adapted from Linden et al. ${ }^{247}$

To further evaluate the putative association of Min8 with CIV, a Min8 $8^{\mathrm{FLAG}}$ yeast strain was used for oxidative cysteine cross-linking (see subsection 2.2.15). Since Min8 contains one cysteine residue at position 36, it was sought to identify interaction partners by $\mathrm{CuSO}_{4}$ treatment. $\mathrm{Cu}(\mathrm{II})$ ions oxidize thiol groups of cysteines and trigger formation of disulfide bonds ${ }^{281}$. After cross-linking cysteines to cysteines by $\mathrm{CuSO}_{4}, \mathrm{Min} 8^{\mathrm{FLAG}}$ was immunoprecipitated and Western blotted (see subsections 2.2.7 and 2.2.9). Distinct bands that were shifted by several kDa were detected (Figure 18a). The shifts occurred most

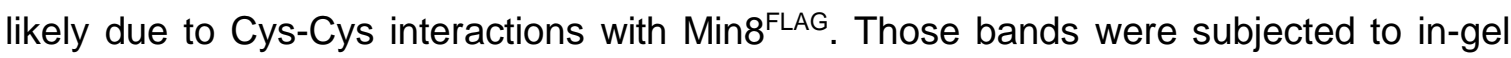
digestion (see subsection 2.3.3) and subsequently analyzed by LC-MS/MS. All identified proteins are listed in Supplementary Table 4 provided on a CD-ROM attached to the hardcopy version of this thesis. Among the identified proteins, Rcf1, Rcf2 and Cox 2 as well as Cyc1 were identified, all of them related to CIV. Co-precipitating proteins, i.e. proteins 
that were identified but not shifted together with Min8, were Cox4, Cox6 and Cox5a, all of them subunits of CIV, as well as Pet9. Other shifted proteins that were also identified are e.g. Tim11, a subunit of CV, and Mic26, part of the mitochondrial contact-site and cristaeorganizing system (MICOS). Combining the BS3- and $\mathrm{Cu}(\mathrm{II})$-cross-links provide a comprehensive Rcf-Pet9-OXPHOS interaction network in which Min8 seems to participate (Figure 18b).

a

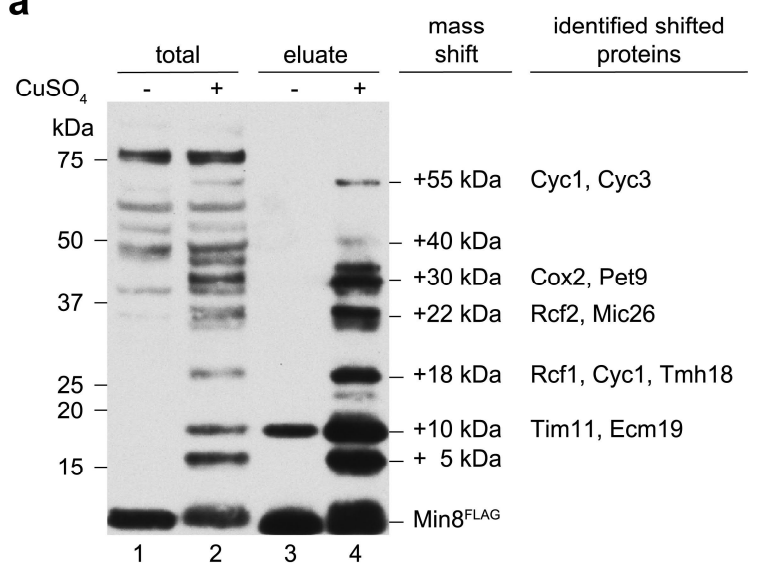

b

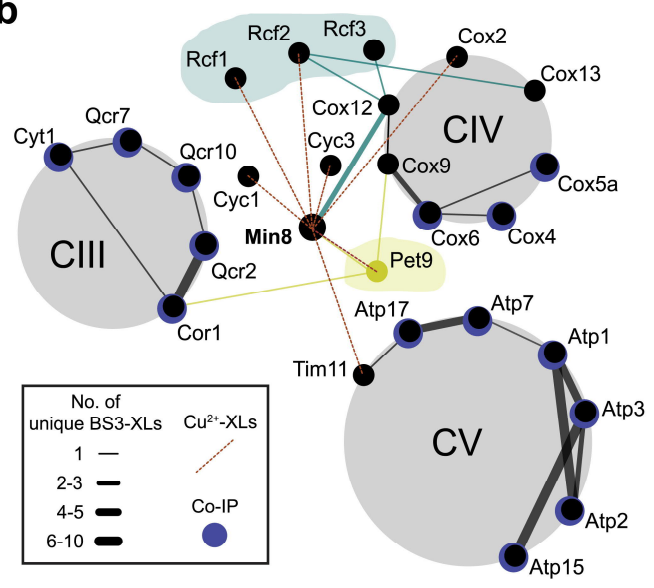

Figure 18: Association of Min8 to the cytochrome $c$ oxidase. (a) Western blot of Min8FLAG immunoprecipitation with anti-FLAG antibody before and after $\mathrm{CuSO}_{4}$-induced cysteine-to-cysteine cross-linking, total input and eluted from beads. Mass shifts and a representation of identified shifted proteins are indicated. (b) Schema of identified Min8-Rcf-Pet9-OXPHOS cross-linking network. Black lines, BS3 cross-links; cupreous, potential $\mathrm{CuSO}_{4}$ links. Blue circles, coimmunoprecipitated but not shifted proteins. Thickness of the lines is proportional to unique residueto-residue cross-links. IMS, intermembrane space; IM, inner membrane.

These results indicate that Min8 could be a novel structural subunit of CIV. To corroborate this hypothesis, Min8 or Cox12 were immunoprecipitated after cross-linking with BS3 in combination with Western blotting. This showed a direct interaction between Min8 and Cox12. Next, a functional relevance of the association of Min8 to CIV was investigated by generating a min $8 \Delta$ strain. These cells did not show any growth defects, neither under fermentative nor under non-fermentative conditions. With special focus on CIV it could also be demonstrated that amount and activity of CIV were not affected in min8D cells. According to the cross-links between Min8 and Cox12, it was further investigated whether Min8 influences the late stage assembly of Cox12 and Cox13, both positioned at the periphery of the complex. Protein import assays (see subsection 2.2.6) with radiolabeled Cox12 and Cox13 indicated that assembly of Cox13 to CIV was slightly increased in the absence of Min8. Assembly of Cox12, in turn, was hampered. Surprisingly, Cox12 was detected in a yet undefined complex that was also observable in a cox $4 \Delta$ mutant that 
3.2 Cross-linking combined with mass spectrometry of mitochondria derived from a rho 0 yeast strain

should not contain any mature CIV. Hence, Min8 seems to support the assembly of Cox12 into an intermediate complex. This work is described in more detail in the $\mathrm{PhD}$ thesis with the preliminary title 'Regulation of mitochondrial supercomplex formation' performed by Bettina Homberg, Cellular Biochemistry, University Medical Center Göttingen. Bettina Homberg is a PhD student in the GGNB program 'Molecular Medicine'.

\subsection{Cross-linking combined with mass spectrometry of mitochondria derived from a $r h o^{0}$ yeast strain}

The initial idea of using the $r o^{\circ}$ strain for cross-linking experiments was to reduce sample complexity, and, hence, to improve cross-linking results. It was anticipated to gain more detailed insights of protein-protein cross-links between mitochondrial proteins and protein complexes. Since the rho ${ }^{\circ}$ strain does not contain mitochondrial DNA (mtDNA), the core proteins Cob and Cox1-3 of the ETC, subunits Atp6, Atp8 and Oli1 of the $F_{1} F_{0}$ ATP synthase as well as ribosomal $15 \mathrm{~S}$ and $21 \mathrm{~S}$ RNAs are not translated ${ }^{37}$. Consequently, abundant protein complexes in wild type yeast strains such as complex III and complex IV of the ETC and the mitoribosome are not assembled correctly and should not be involved in cross-linking reactions. In theory, this should increase identifications of intraprotein and interprotein cross-links in other, also low abundant, protein complexes such as the translocases of outer and inner membrane (TOM/TIM) complexes or the mitochondrial contact-site and cristae-organizing system (MICOS), amongst others. Mitochondria from the $r h o^{\circ}$ yeast strain were isolated and purified by Dr. Markus Deckers and Mirjam Wissel, Cellular Biochemistry, University Medical Center Göttingen. Cross-linking reactions and data acquisition were performed by Dr. Chung-Tien Lee during his time as a postdoctoral researcher in the Bioanalytical Mass Spectrometry Group at the Max Planck Institute for Biophysical Chemistry.

With respect to identified CSMs, cross-linked proteins, and unique residue-toresidue cross-links, the analysis of cross-linked mitochondria derived from the rho ${ }^{0}$ strain resulted in comparable numbers as the ones provided by the glycerol and glucose data sets (see subsection 3.1.2, Table 6). In the $r \mathrm{O}^{\circ}$ data set, OM-to-OM protein-protein crosslinks accounted for $14 \%$, nearly twice the number compared to the glycerol and glucose data sets (Figure 19). Additionally, IM-to-IM protein-protein cross-links were reduced by almost the half to $26 \%$ what is in line with the fact that in the $r o^{\circ}$ strain complexes within the ETC - an integral part of the IM - are not assembled correctly. M-to-M protein-protein cross-links with $25 \%$ were equally present compared to the other data sets. Surprisingly, cross-links between proteins that were not assigned to mitochondria were relatively high $(14 \%)$ and could be due to contaminations during the isolation process. All identified 
cross-links are listed in Supplementary Table 2 provided on a CD-ROM attached to the hardcopy version of this thesis.

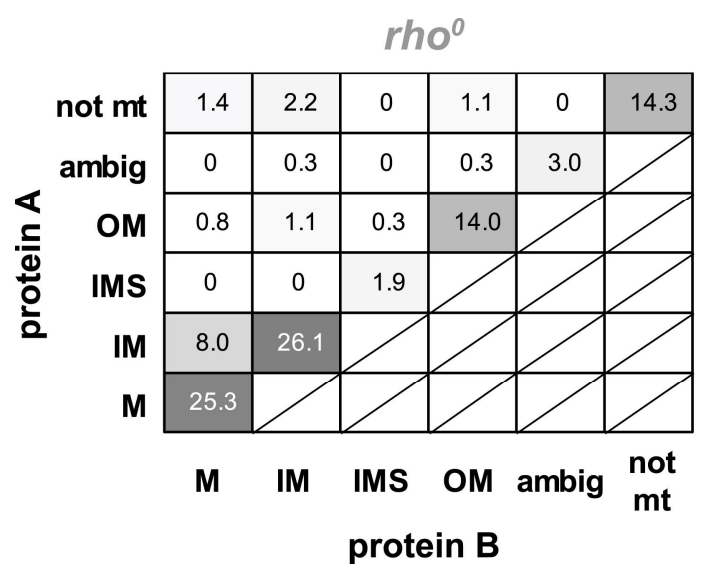

Figure 19: Relative distribution of protein-protein cross-links after cross-linking mitochondria derived from the $r h 0^{\circ}$ yeast strain according to their subcompartmental localization. Intraprotein and interprotein cross-links were considered. Localization assignments are based on Vögtle et al. ${ }^{142}$ OM, outer membrane; IMS, intermembrane space; IM, inner membrane; M, matrix; ambig, ambiguous; not mt, not mitochondrial.

\subsubsection{Cross-links within the OXPHOS system and the PDH}

Analyzing the identified cross-links within the OXPHOS system of the rho $0^{0}$ strain revealed only a low number of cross-links within and between the complexes (Figure 20a) compared to the identified cross-links in mitochondria derived from yeast grown on glycerol- or glucose-containing medium (see subsection 3.1.5, Figure 10). Cross-links between subunits of CII, namely Sdh1-3, were also covered in the $r h 0^{\circ}$ data set, whereas interactions within CIII-CV were significantly reduced. Cross-links between Cor1 and Qcr2 were identified within CIII, cross-links between Cox5b and Cox6 within CIV. No cross-links between subunits of CIII and CIV were detected. Pet9 was also not part of any identifiable cross-link to the OXPHOS system. This is in line with the lack of mtDNA in the rho ${ }^{0}$ strain that therefore does not show a functional ETC.

Cross-links within the PDH were identified and quantified in mitochondria derived from yeast grown on glycerol- or glucose-containing medium (see subsection 3.1.7) indicating that fully assembled PDH complexes are more abundant when glucose is metabolized. Accordingly, also in the $r h o^{\circ}$ data set (the $r h o^{\circ}$ strain only survives on media containing fermentable carbon sources, like glucose) interprotein cross-links between the E3-binding protein $\mathrm{Pdx} 1$ and E2 as well as E3 were identified (Figure 20b). Simultaneously, the number of interprotein cross-links between E1 and E2 was increased compared to the glycerol condition and comparable to the glucose condition. In 
combination with the quantitative data, these findings indicate that fully assembled PDHs, i.e. where $\mathrm{Pdx} 1$ brings $\mathrm{E} 3$ in close proximity to $\mathrm{E} 2$, are more abundant when glucose is used as carbon source.

a

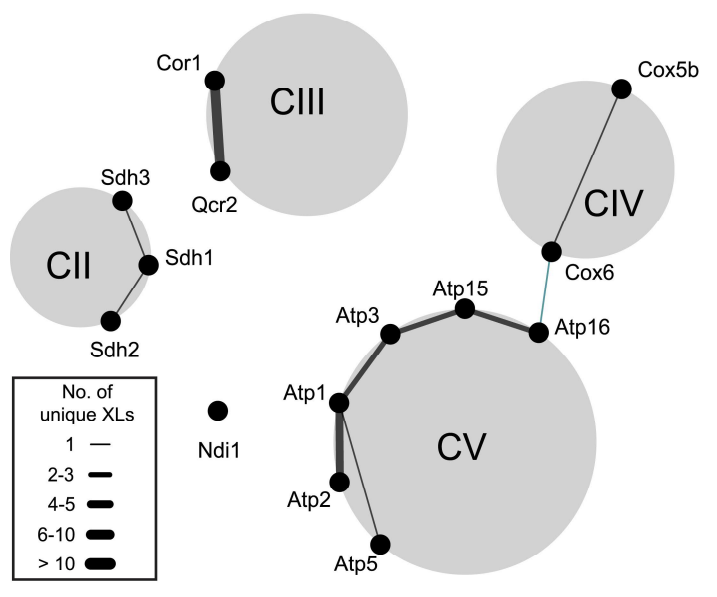

b

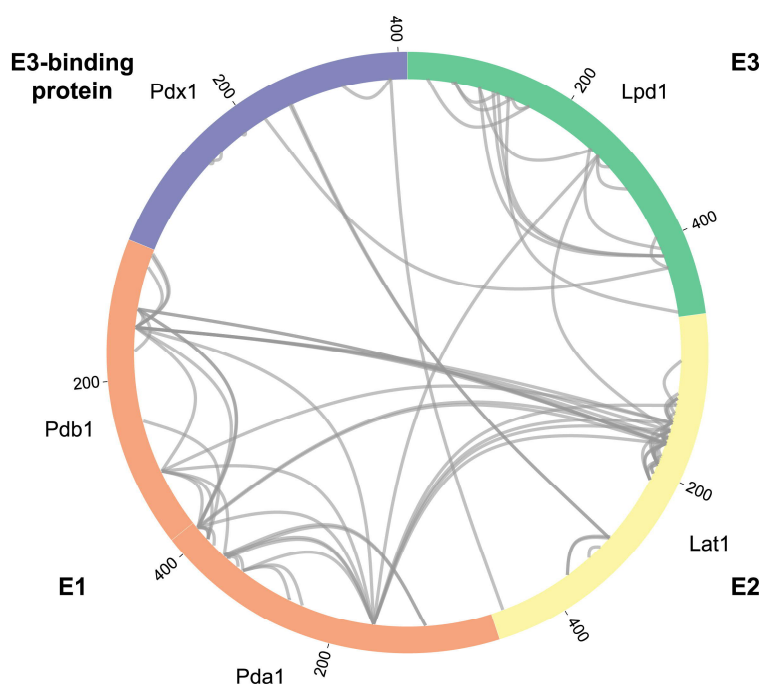

Figure 20: Cross-links within the OXPHOS system and the pyruvate dehydrogenase complex $(\mathrm{PDH})$ identified in mitochondria derived from the $r \mathrm{O}^{\circ}$ yeast strain. (a) Interprotein cross-links within the OXPHOS system. Ndi1 and $F_{1} F_{0}$ ATP synthase (CV) are included. Thickness of the edges is proportional to the number of unique residue pairs. Black lines, intra-complex cross-links; turquoise lines, inter(-complex) cross-links. (b) Cross-links within the PDH. Visualized by $x V^{2}{ }^{271}$.

\subsubsection{Cross-links within the TCA cycle}

All proteins involved in the tricarboxylic acid (TCA) cycle could be identified in cross-linked mitochondria from $r h o^{\circ}$ yeast cells. This comprehensiveness could not be achieved in the glycerol and glucose data sets. The citrate synthase (Cit1), the aconitase (Aco1), the isocitrate dehydrogenase (Idh1, Idh2), the a-ketoglutarate dehydrogenase (Kgd1, Kgd2, Lpd1), the succinyl-CoA synthase (Lsc1, Lsc2), the succinic dehydrogenase (CIl of the ETC; Shd1, Sdh2, Sdh3), the fumarase (Fum1) and the malate dehydrogenase (Mdh1) were cross-linked (Figure 21, red background). While most of them were only detected by intraprotein cross-links, interactions between Aco1 and Idh2 were identified. These proteins are involved in two consecutive reaction steps within the TCA cycle. Aconitase catalyzes the isomerization of citrate to isocitrate that is subsequently oxidized and carboxylated to $\alpha$-ketoglutarate by isocitrate dehydrogenase. Since the decarboxylation step is rate-limiting, a tight connection between aconitase and isocitrate dehydrogenase could accelerate substrate channeling. Other interprotein cross-links between proteins of 
the TCA cycle could not be detected, although the existence of the 'metabolon', i.e. the supercomplex of proteins involved in the TCA cycle, was already described by crosslinking and mass spectrometry ${ }^{282}$. Multiple residue-to-residue cross-links between Aco1 and Ilv5, the mitochondrial ketol-acid reductoisomerase, were identified. Ilv5 is part of the isoleucine/valine biosynthesis pathway. This pathway consists of four steps, including six different proteins. Four of these six proteins were identified in the rho data set (Ilv6, Ilv2, Ilv5, Ilv3), showing also interprotein cross-links within this pathway (Figure 21, blue background). The two missing proteins, Bat1 and Bat2, are involved in a last transamination step. Instead, a protein that has been described to regulate the transamination step could be identified, Mmf1 ${ }^{283}$. Mmf1 also provided cross-links to Aco1. The interactions of Ilv5 to Aco1 and additionally to the isocitrate dehydrogenase as well as Mmf1 to Aco1 link this amino acid biosynthesis pathway to the TCA cycle.

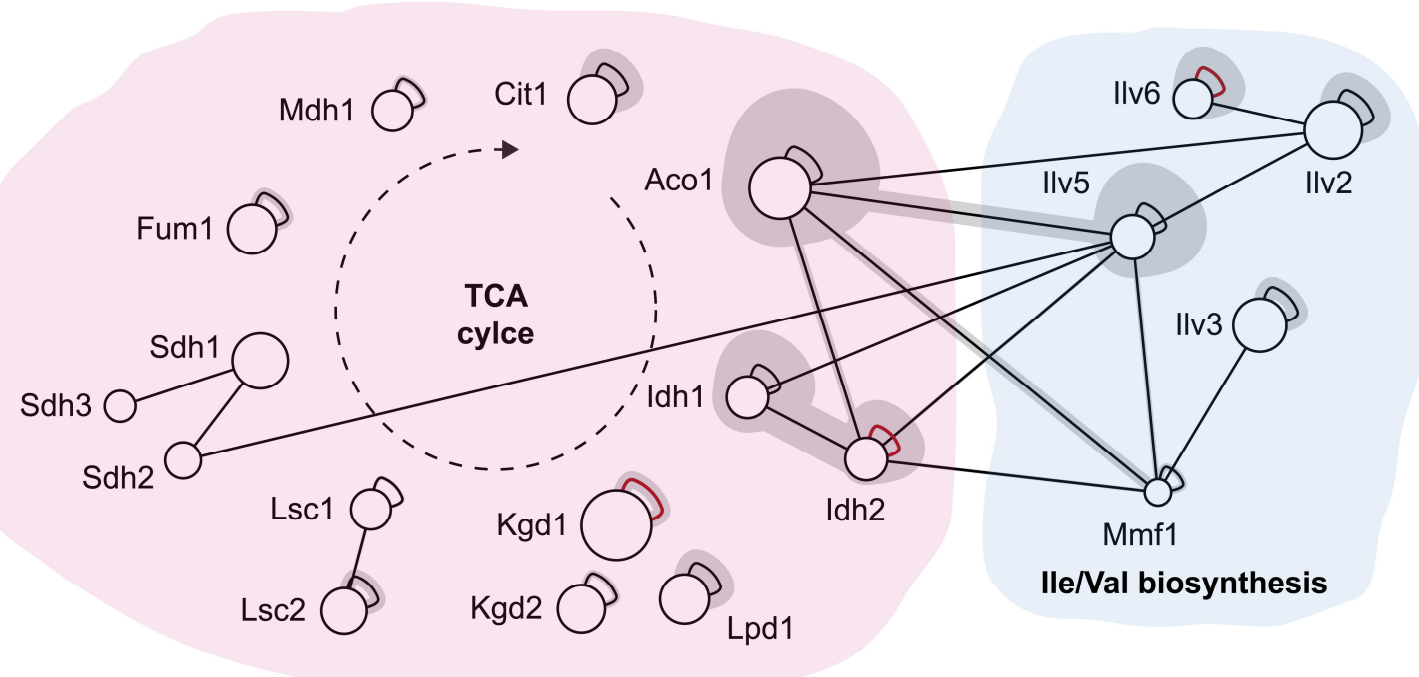

Figure 21: Identified cross-links within the TCA cycle and its connection to proteins involved in amino acid biosynthesis in mitochondria derived from $r h o^{\circ}$ yeast cells. Circles represent proteins, circle size is proportional to protein size. Edges indicate cross-links, thickness is proportional to unique residue-to-residue cross-links. Edges to the same protein illustrate intraprotein cross-links, edges between two proteins indicate interprotein cross-links. Red-colored edges represent crosslinks within one protein but to the same residue, indicating a multimeric protein structure. Red background, proteins belonging to the TCA cycle; blue background, proteins belonging to the isoleucine/valine biosynthesis pathway. Network visualized by xiNET ${ }^{270}$.

\subsubsection{Cross-links within TOM/TIM complexes}

A major rationale of using the $r h 0^{\circ}$ strain was to determine interactions within the complexes of the translocases of the outer and inner membrane (TOM/TIM). The TOM/TIM system supports the transport of proteins into the mitochondrion and consists of 
at least 27 directly involved proteins ${ }^{21}$. Indeed, the number of CSMs supporting unique residue-to-residue cross-links of proteins involved in the TOM/TIM complexes was almost doubled when compared to the glucose and glycerol data sets (176 CSMs in the $r o^{\circ}$ data set versus 88 and 97 in the glucose and glycerol data set, respectively). However, only Tom5 and its cross-link to Por 1 could be exclusively identified in the $r h 0^{\circ}$ data set as part of the TOM system (Figure 22). Besides that, cross-links between Por1 and Tom40 could be identified under glycerol and glucose conditions as well as in the $r o^{\circ}$ strain. Tom40 forms the membrane pore in the OM and is a major constituent of the TOM complex ${ }^{284}$. Furthermore, also the TIM10 complex consisting of Tim9 and Tim10 was identified in all conditions. These two soluble proteins are an essential part in protein import and are located in the IMS ${ }^{285,286}$. Additionally, the TIM23 complex was covered as well in all conditions, mainly by cross-links within the PAM complex. In this study, interprotein crosslinks between Pam18, Pam16 and Tim44 as well as Ssc1 and Mge1 could be identified. The PAM complex shows ATPase activity and supports the import of matrix proteins ${ }^{287}$. In summary, some parts of the TOM/TIM complexes could be covered by this cross-linking approach (for a full list see Supplementary Table 7), but the initial hypothesis that the $r h o^{\circ}$ strain might provide a deeper insight into these complexes did not prove true.

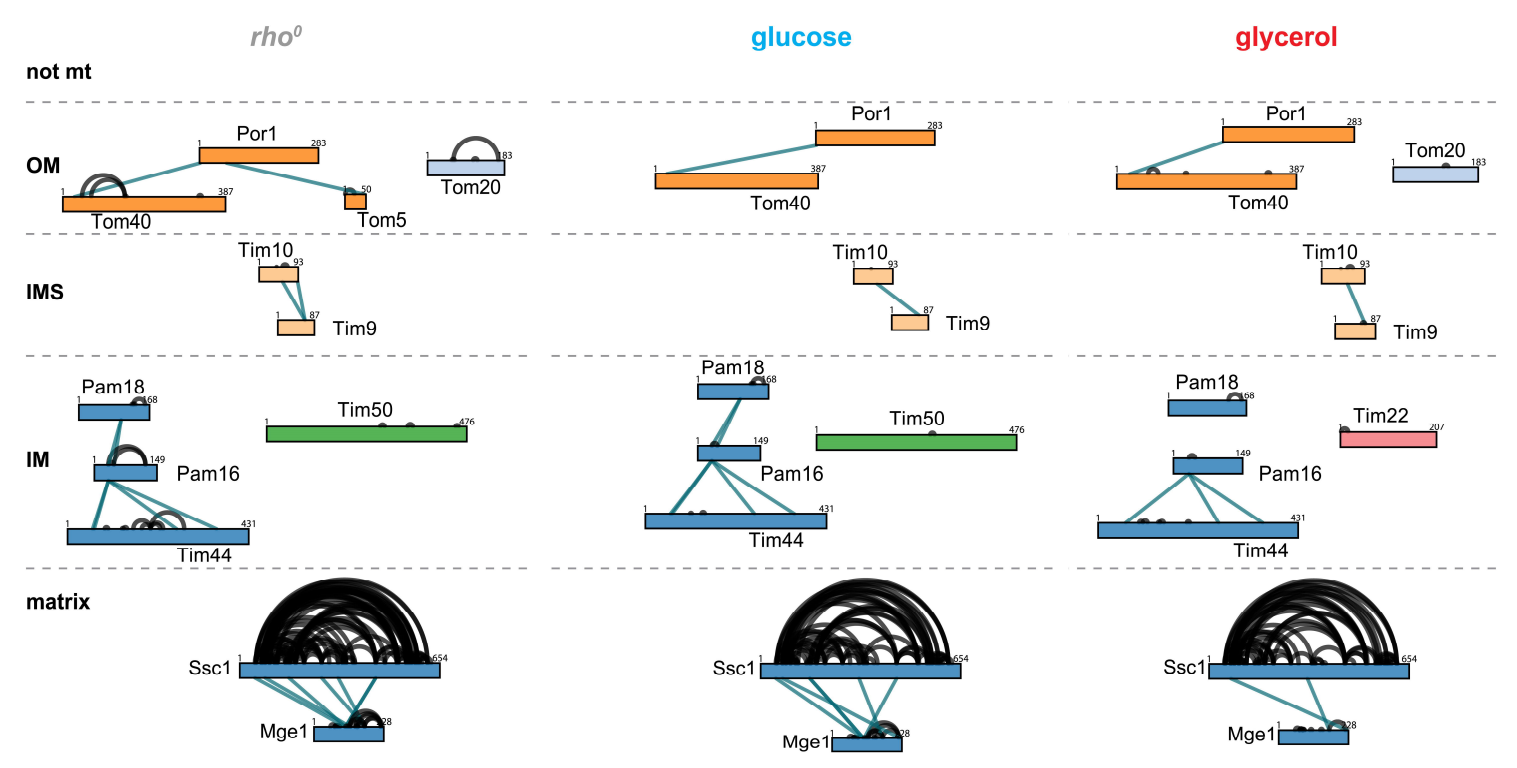

Figure 22: Cross-linking network of TOM/TIM proteins identified in mitochondria derived from rho ${ }^{\circ}$ yeast cells as well as from wild type mitochondria derived from yeast grown on glycerol- or glucosecontaining medium. Turquoise lines, interprotein cross-links; black lines, intraprotein cross-links; not mt, not mitochondrial; OM, outer membrane; IMS, intermembrane space; IM, inner membrane. Networks visualized by $x$ Vis 271 . 


\subsection{Protein size exclusion chromatography as additional fractionation step to improve analysis depth of cross-linked proteins}

Additional protein and peptide fractionation steps in MS-based proteomics reduce the sample complexity and increase the analysis depth of the final LC-MS/MS analyses. A drawback of additional sample preparation steps is the increased analysis time. Since protein size exclusion chromatography (protein SEC) of cross-linked proteins and protein complexes derived from cross-linked mitochondria from yeast grown on glycerolcontaining medium has been performed as a prefractionation step, the data were annotated and evaluated in this thesis and compared with data obtained by the peptide SEC workflow (see subsection 3.1.2). Protein SEC under denaturing conditions $(0.1 \%$ SDS) was performed after lysis of cross-linked mitochondria (see subsection 2.2.16). Fractions containing cross-linked proteins were digested with the endoproteinase trypsin and then subjected to peptide SEC. Fractions containing the cross-linked peptides were analyzed by LC-MS/MS. Mitochondria from yeast grown on glycerol-containing medium were isolated and purified by Dr. Markus Deckers and Mirjam Wissel, Cellular Biochemistry, University Medical Center Göttingen. Cross-linking reactions, protein SEC, and data acquisition were performed by Dr. Chung-Tien Lee during his time as a postdoctoral researcher in the Bioanalytical Mass Spectrometry Group at the Max Planck Institute for Biophysical Chemistry.

Table 7 compares the results of the analyses using the protein SEC approach versus the peptide SEC approach. Using protein SEC as additional fraction step revealed more CSMs as compared to the peptide SEC approach, both in non-filtered and filtered

Table 7: Numbers of total identified cross-linked peptides spectrum matches (CSMs), cross-linked proteins, unique residue-to-residue cross-links and the interprotein cross-links in percent for the unfiltered and the filtered data set for peptide SEC and the protein SEC approach. 'Single CSMs' in percent represent those unique residue-to-residue cross-links that were identified only by a single CSM.

\begin{tabular}{|c|c|c|c|c|}
\hline & $\begin{array}{r}\text { unfil } \\
\text { glycerol } \\
\text { peptide SEC }\end{array}$ & $\begin{array}{l}\text { red } \\
\text { glycerol } \\
\text { protein SEC }\end{array}$ & $\begin{array}{r}\text { filte } \\
\text { glycerol } \\
\text { peptide SEC }\end{array}$ & $\begin{array}{l}\text { ed } \\
\text { glycerol } \\
\text { protein SEC }\end{array}$ \\
\hline total CSMs & 25022 & 53646 & 23859 & 52012 \\
\hline $\begin{array}{l}\text { cross-linked } \\
\text { proteins }\end{array}$ & 315 & 424 & 261 & 308 \\
\hline $\begin{array}{l}\text { unique residue-to- } \\
\text { residue cross-links }\end{array}$ & 2999 & 2789 & 2100 & 1957 \\
\hline $\begin{array}{l}\text { interprotein cross- } \\
\text { links }\end{array}$ & $22.2 \%$ & $29.1 \%$ & $17.1 \%$ & $19.1 \%$ \\
\hline single CSMs & $29.7 \%$ & $29.2 \%$ & & \\
\hline
\end{tabular}


(CSMs must have a spectrum score greater than four and residue-to-residue cross-links must be supported by more than one CSM) data sets. Also, the number of intraprotein and interprotein cross-links increased. However, the numbers of unique residues-to-residues cross-links did not increase but was surprisingly lower. The number of proteins that were involved in cross-linking reactions increased from 315 to 424 (unfiltered). All identified cross-links are listed in Supplementary Table 3 provided on a CD-ROM attached to the hardcopy version of this thesis.

86 protein-protein cross-links were exclusively identified by the protein SEC approach; $94 \%$ (81) of them represented by only a single residue-to-residue cross-link. These results indicate that the additional fractionation step did not significantly increase the number of protein-protein cross-links when compared to the results from the peptide SEC approach. Nonetheless, protein SEC added valuable information to protein-protein cross-links that were already identified by the peptide SEC approach. For example, another residue-to-residue cross-link between Min8 and Cox12 was identified. K69 of Min8 cross-linked not only to K41 and the N-terminus of Cox12 on the IMS-side but also to K53 of Cox12, thus supporting the 3D model of CIV with integrated Min8 (see subsection 3.1.10). Besides that, additional cross-links within the OXPHOS complexes were identified (Table 8). An interprotein cross-link between CIII and CIV underlines the existence of an ETC supercomplex (Cor1 to Cox1). Furthermore, interprotein cross-links between CIII and CV were identified with the protein SEC approach.

Table 8: Protein-protein cross-links within the OXPHOS system of mitochondria derived from yeast grown on glycerol-containing medium uniquely identified via the protein SEC approach. \#unique, number of unique residue-to-residue cross-links contributing to the particular protein-protein crosslink; \#CSMs, number of cross-linked peptides spectrum matches supporting the particular proteinprotein cross-link.

\begin{tabular}{lrrrr} 
complex & Protein 1 & Protein 2 & \#unique & \#CSMs \\
\hline CII & Sdh1 & Sdh5 & 3 & 23 \\
CII & Sdh2 & Sdh9 & 1 & 2 \\
CIII-CIV & Cor1 & Cox1 & 1 & 3 \\
CIII-CV & Qcr2 & Atp16 & 1 & 5 \\
CIII-CV & Qcr7 & Atp5 & 1 & 2 \\
CIV & Cox2 & Cox12 & 1 & 12 \\
CIV & Cox6 & Cox12 & 1 & 2 \\
CV & Atp2 & Atp15 & 1 & 3 \\
CV & Atp4 & Atp17 & 1 & 4
\end{tabular}


Other protein-protein cross-links exclusively identified after protein SEC are shown in Figure 23. Cross-links from proteins with so far unknown function to other proteins were identified, such as Fmp10 to Mrx3, Fmp40 to Ald4 and Fmp41 to Hsp77. Furthermore, cross-links between OM proteins and proteins that are not assigned to mitochondria were detected, such as Om45 to Sss1 and Por1 to Min6. Sss1 is a subunit of the Sec61 translocation complex of the endoplasmic reticulum $(E R)^{288}$. Min6 is a protein of unknown function and is assigned to peroxisomes ${ }^{289}$. These cross-links might indicate the connection between mitochondria and other organelles. Cross-links between Tim11, a subunit of CV, and Mic10, component of MICOS, support the interaction of both complexes which has been previously described ${ }^{19,290}$. Another cross-link was detected between Cox13 and Mia40, the mitochondrial import and assembly protein ${ }^{58}$. In summary, the prefractionation step with protein SEC resulted in more CSMs and confirmed cross-links identified by the peptide SEC approach but also added information of hitherto not described protein interactions. Unfortunately, protein SEC prefractionation did not reveal additional cross-links of the TOM/TIM complexes.

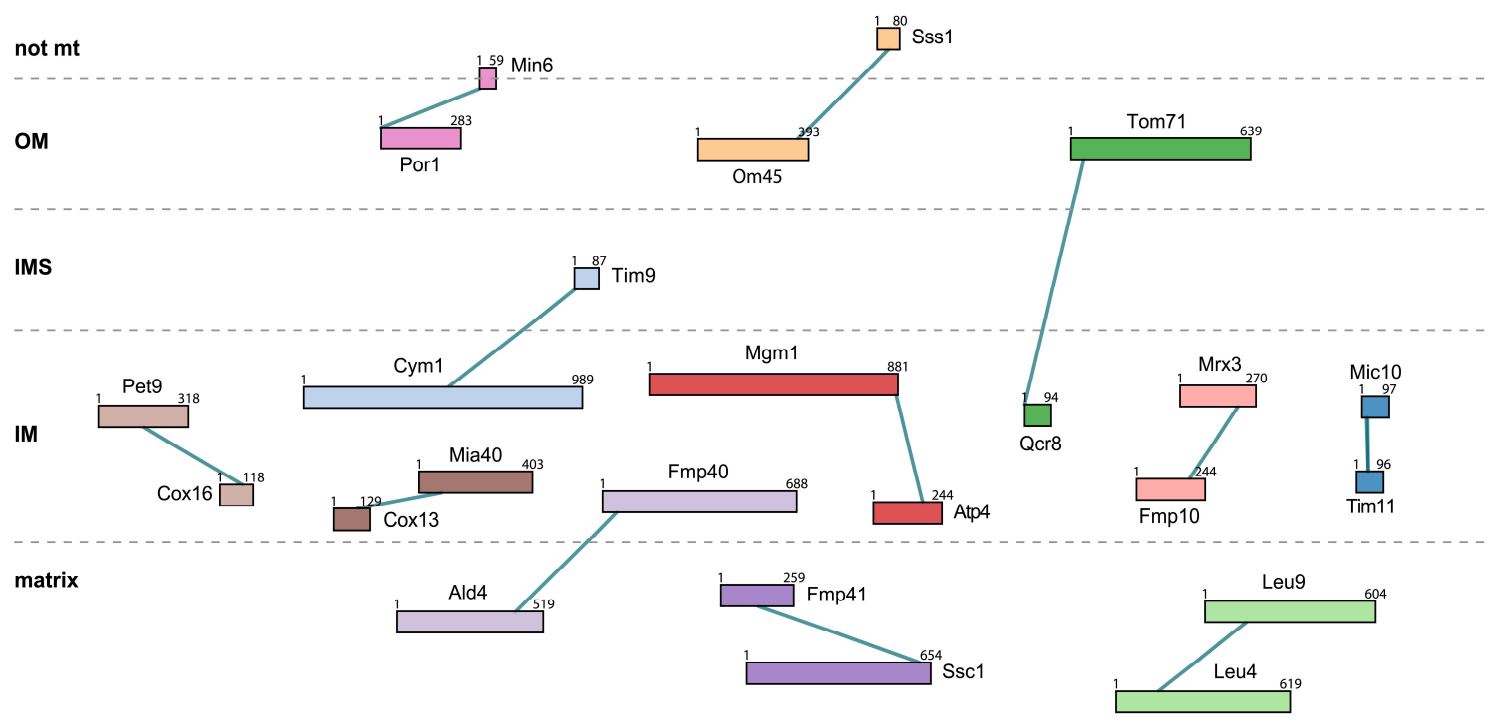

Figure 23: Selected protein-protein cross-links uniquely identified in the protein SEC approach after cross-linking mitochondria derived from yeast grown on glycerol-containing medium. Thickness of the edges is proportional to the number of unique residue-to-residue cross-links. Turquoise lines, interprotein cross-links; not $\mathrm{mt}$, not mitochondrial; OM, outer membrane; IMS, intermembrane space; IM, inner membrane. 
3.4 Proteomic analysis of mitochondria derived from yeast grown on glycerol- or glucosecontaining medium and from the rho0 yeast strain

\subsection{Proteomic analysis of mitochondria derived from yeast grown on glycerol- or glucose-containing medium and from the $r h o^{\circ}$ yeast strain}

Yeast cells grown on fermentable and non-fermentable carbon sources adapt their metabolism according to the provided supply ${ }^{291-293}$. Also, the amount and morphology of yeast's mitochondria change depending on the carbon source $35,294,295$. This comes along with differences in protein abundances ${ }^{72}$. The same holds true for the rho yeast strain that lacks mitochondrial $\mathrm{DNA}^{296,297}$. In this strain the mitochondrial ribosome (mitoribosome) and the electron transport chain (ETC) are not assembled correctly, because cells cannot synthesize core proteins of ETC complexes and also rRNAs necessary for mitoribosome assembly ${ }^{37}$. Based on these changes, comparison of protein abundances by MS-based proteomics should aid distinguishing between the mitochondria from different strains and growth conditions. In this regard, a comparative protein identification search with MaxQuant ${ }^{254}$ of all the fractions resulting from peptide SEC replicates of all conditions was performed (see subsection 2.4.2.1). By performing principal component analysis (PCA) via Perseus ${ }^{272}$, replicates belonging to mitochondria isolated either from yeast grown on glycerol, glucose or the $r h o^{\circ}$ strain could be separated (Figure 24).

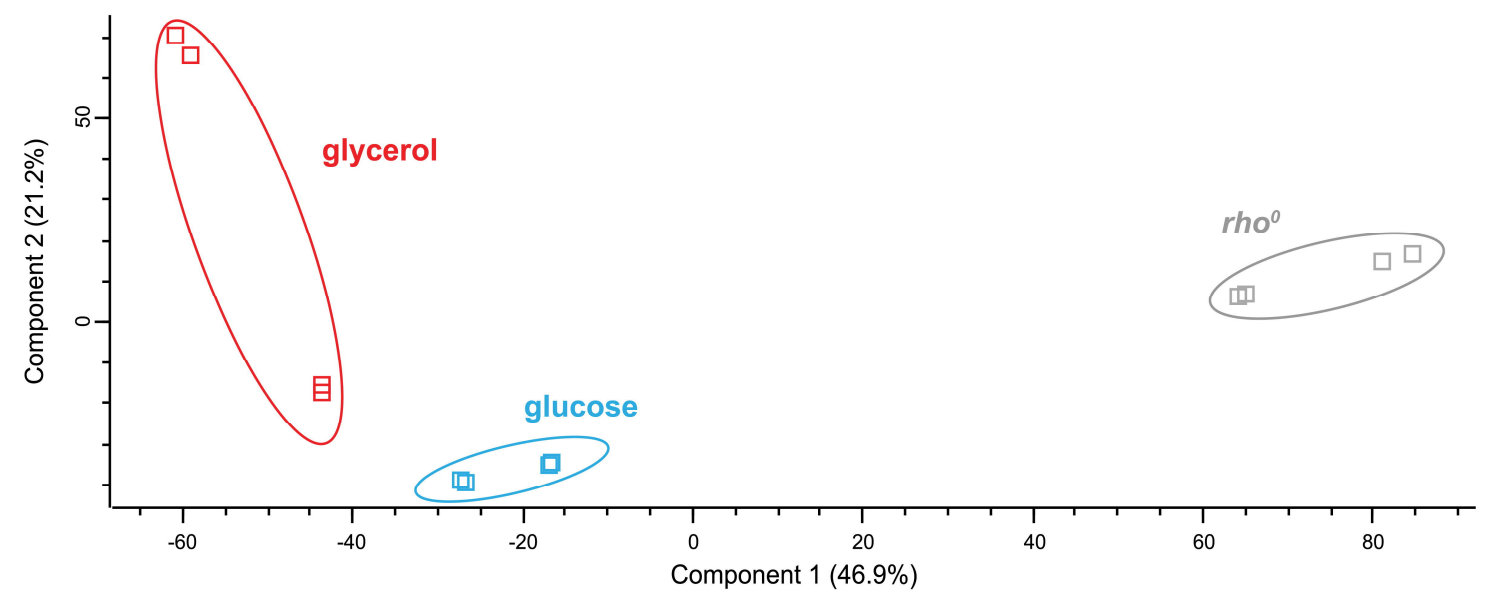

Figure 24: Principal component analysis (PCA) of glycerol, glucose and rho condition. PCA is based on identified linear, i.e. non-cross-linked peptides. Displayed are technical replicates, four per condition, that could be separated depending on carbon source and strain. Red, glycerol condition; blue, glucose condition; gray: rho ${ }^{\circ}$ strain. Calculated and illustrated by Perseus ${ }^{272}$.

Proteins from the mitoribosome like Mrp7, Mrpl10 and Mrp20 showed equal abundances in mitochondria from yeast grown on glycerol and glucose in all replicates but were less abundant in the rho strain (data not shown). When focusing on the comparison of mitochondria from yeast derived from glycerol and glucose growth, proteins belonging to the ETC like Sdh1, Qcr6 and Cox1 or the glycerol-3-phosphate dehydrogenase Gut2 
showed higher abundances in the glycerol condition whereas proteins belonging to amino acid biosynthesis pathways such as Lys4 or Arg5,6 showed higher abundances in the glucose condition (data not shown). These results reflect the metabolic condition of the yeast and are in agreement with other proteomic studies dealing with mitochondria derived from yeast grown on different carbon sources ${ }^{9,72,146}$ and are in line with the presented cross-linking results.

\subsection{Quality control of yeast mitochondria}

To investigate the quality of purified yeast mitochondria prior to cross-linking, several control experiments were performed to investigate the quality of purified yeast mitochondria prior to cross-linking. To assess the influence of the sucrose gradient and the subsequent freezing on the performance of mitochondria, the oxygen consumption (see subsection 3.5.1), the import quality (see subsection 3.5.2), the activity of respiratory chain complexes (see subsection 3.5.3) and the membrane integrity (see subsection 3.5.4) were tested. As references, freshly isolated crude mitochondrial extract, frozen crude mitochondrial extract and/or freshly purified mitochondria and frozen purified mitochondria were considered. All these experiments were performed and analyzed by Dr. Markus Deckers, Bettina Homberg, Dr. Tobias Müller and Mirjam Wissel, Cellular Biochemistry, University Medical Center Göttingen. Bettina Homberg is a PhD student in the GGNB program 'Molecular Medicine'.

\subsubsection{Oxygen consumption of isolated and purified yeast mitochondria}

The Oroboros O2k system measures the oxygen concentration in defined chambers by an oxygen sensor and translates it into an oxygen flux. With this system mitochondria's ability to perform oxidative phosphorylation (OXPHOS) can be measured, since the OXPHOS process consumes oxygen. For that, freshly isolated crude mitochondrial extract, frozen crude mitochondrial extract, freshly purified mitochondria and frozen purified mitochondria in a defined amount were placed into the Oroboros O2k chamber (see subsection 2.2.5). Pyruvate and malate were added as substrates of the TCA cycle. Within the TCA cycle, redox equivalents are produced that are necessary for the final reduction of oxygen to water at complex IV of the respiratory chain. With this, the basal respiration of the mitochondria was measured. Here, a decrease of oxygen flux between the crude mitochondrial extract and the purified mitochondria by almost two-thirds was measured (Figure 25). Also, oxygen flux was slightly decreased between fresh and frozen material. ADP was added as substrate for complex V, the ATP synthase, that uses ADP during the 
OXPHOS process formed proton gradient to produce ATP. In theory, this should boost oxygen flux, since ATP synthase is saturated and OXPHOS can reach maximum respiration capacity, termed "state 3". This effect was observed for crude mitochondrial extracts and very slightly for the freshly purified mitochondria. Again, oxygen flux was decreased when comparing fresh with frozen material. In a next step, oligomycin was added, resulting in an inhibition of ATP synthase. The measured oxygen flux was due to leaks in the inner mitochondrial membrane, called leaky respiration. The trend continued that higher oxygen flux was measured in crude mitochondrial extracts than in purified mitochondria, also the discrepancy between fresh and frozen material. FCCP was added to uncouple the respiration from the inhibited ATP synthase. This should lead to maximum respiration but did not affect the oxygen flux in all yeast mitochondria samples. As last step, antimycin A as CIII inhibitor was added to block respiration. Consequently, oxygen flux in all samples dropped close to zero. Mitochondria of all preparations showed the ability to undergo respiration. However, freshly isolated crude mitochondrial extract outperformed mitochondria that were purified by tandem sucrose gradient centrifugation.

Furthermore, fresh material outperformed the frozen one.

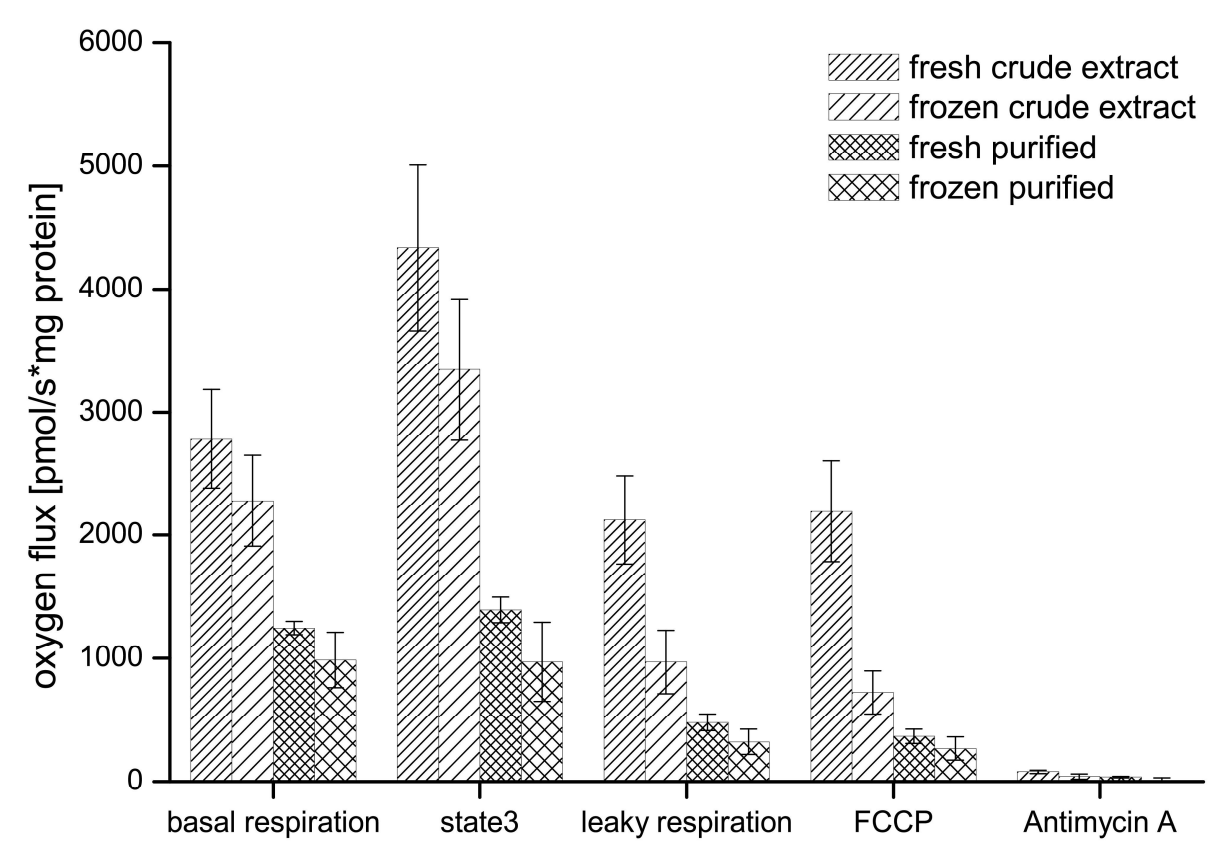

Figure 25: Oxygen flux of isolated and purified yeast mitochondria measured with Oroboros. The oxygen flux in pmol per s and mg protein is plotted for every induced state within the Oroboros O2k respirometry assay. Basal respiration describes the state after providing pyruvate and malate as substrates for the TCA cycle, state3 shows maximal respiration capacity after adding ADP as CV substrate, leaky respiration the oxygen flux after inhibition of CV by oligomycin, FCCP after uncoupling the respiration from CV and antimycin A after inhibition of CIII, completely blocking the respiration. Plotted are the means of three measurements, error bars indicate standard deviation. Experiments were performed by Bettina Homberg, Cellular Biochemistry, University Medical Center Göttingen, PhD student in the GGNB program 'Molecular Medicine'. 


\subsubsection{Import assay with isolated and purified yeast mitochondria}

Atp2, a subunit of the $F_{1} F_{0}$ ATP synthase was ${ }^{35} S$-radiolabeled and selected as a precursor $\left(\left[{ }^{35} \mathrm{~S}\right] \mathrm{F}_{1} \beta\right)$ for investigating the protein import in freshly isolated crude mitochondrial extract, frozen crude mitochondrial extract, freshly purified mitochondria and frozen purified mitochondria (see subsection 2.2.6). For this, respective mitochondria were incubated for 3,7 , and 15 min together with the precursor with functional membrane potential $([\Delta \Psi])$ and, as a negative control, $15 \mathrm{~min}$ with disrupted membrane potential. Afterwards, mitochondria were treated either with proteinase K (PK) or without. After successful import, the targeting sequence of the precursor sequence of Atp2 is cleaved off and the mature Atp2 was distinguishable by a lower molecular weight after Western blotting (Figure 26). In all approaches and regardless of the isolation protocol and storage of the mitochondria an active import could be shown except for these approaches with disrupted membrane potential. This was expected, since the import of Atp2 is dependent on the membrane potential. This experiment indicates that all described batches of mitochondria showed a functional membrane potential and, consequently, inner membrane integrity.

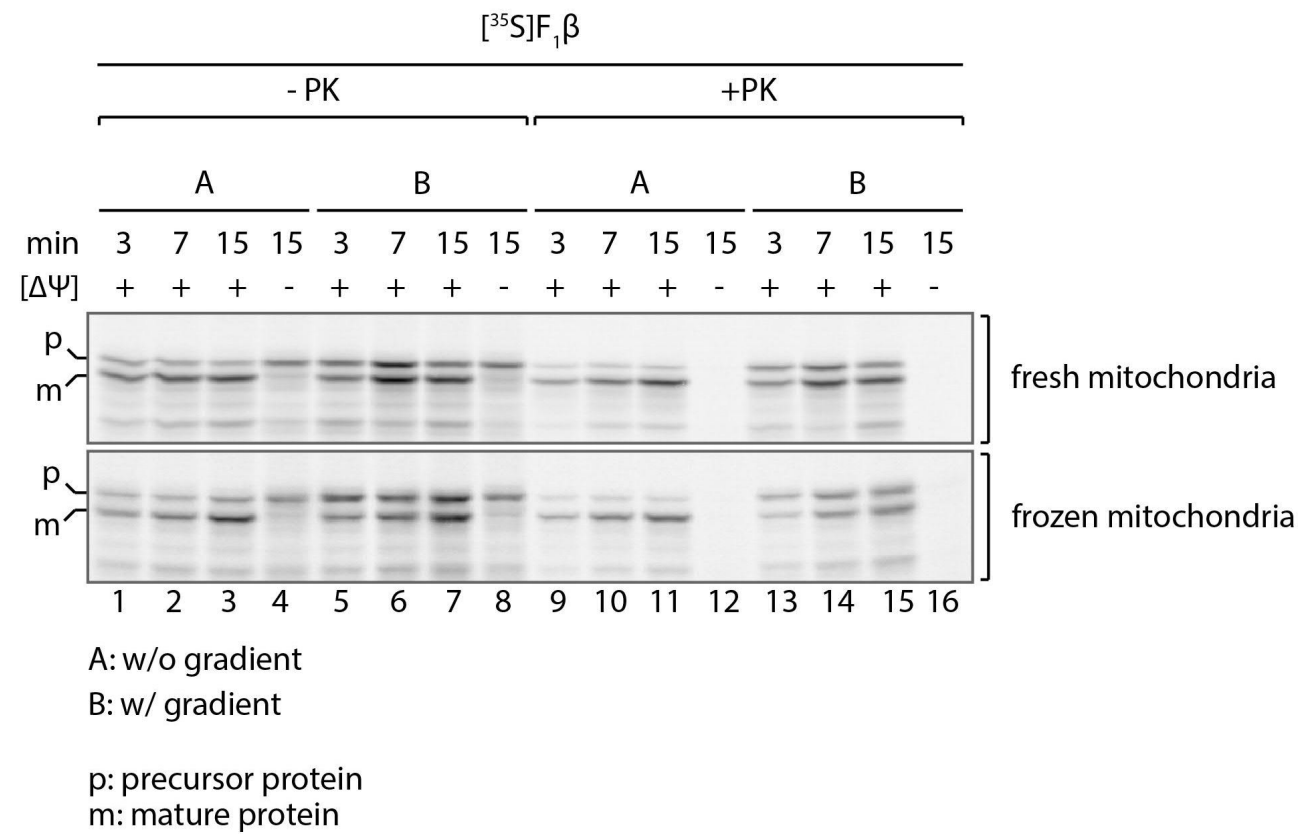

Figure 26: Import assay. Import of ${ }^{35} \mathrm{~S}$-radiolabeled precursor $(p) \operatorname{Atp} 2\left(\left[{ }^{35} S\right] \mathrm{F}_{1} \beta\right)$ in isolated crude mitochondrial extract $(A)$, fresh and frozen, and purified mitochondria $(B)$, fresh and frozen. Time indicates the incubation of precursor and mitochondria with intact membrane potential $([\Delta \Psi])$. After incubation, mitochondria were treated either with proteinase $\mathrm{K}(+\mathrm{PK})$ or without (-PK). Mature Atp2 is indicated by " $m$ ". Experiments were performed and figure was prepared by Dr. Tobias Müller, Cellular Biochemistry, University Medical Center Göttingen. 


\subsubsection{Activity assays to investigate functional integrity of ETC complexes}

Activity assays of complexes II, IV and $\mathrm{V}\left(\mathrm{F}_{1} \mathrm{~F}_{0}\right.$ ATP synthase) were performed to investigate the functional integrity of the ETC in frozen crude mitochondrial extracts and frozen purified mitochondria (see subsection 2.2.8). Therefore, mitochondria were lysed with digitonin and subjected to BN-PAGE. Substrates for the specific complexes (NADH for $\mathrm{Cll}$, reduced cytochrome $c$ for CIV and ATP for CV) were added to the buffered gel slices and combined with a redox-reactive dye (NBT for CII, DAB for CIV and $\mathrm{Pb}\left(\mathrm{NO}_{3}\right)_{2}$ for CV). In case of an active complex the substrate reacted with the complex and, in turn, activated the dye that was finally used as a read-out. Irrespective of the sample preparation, all mitochondria showed active complexes II, IV and V (Figure 27), indicating a functional ETC.

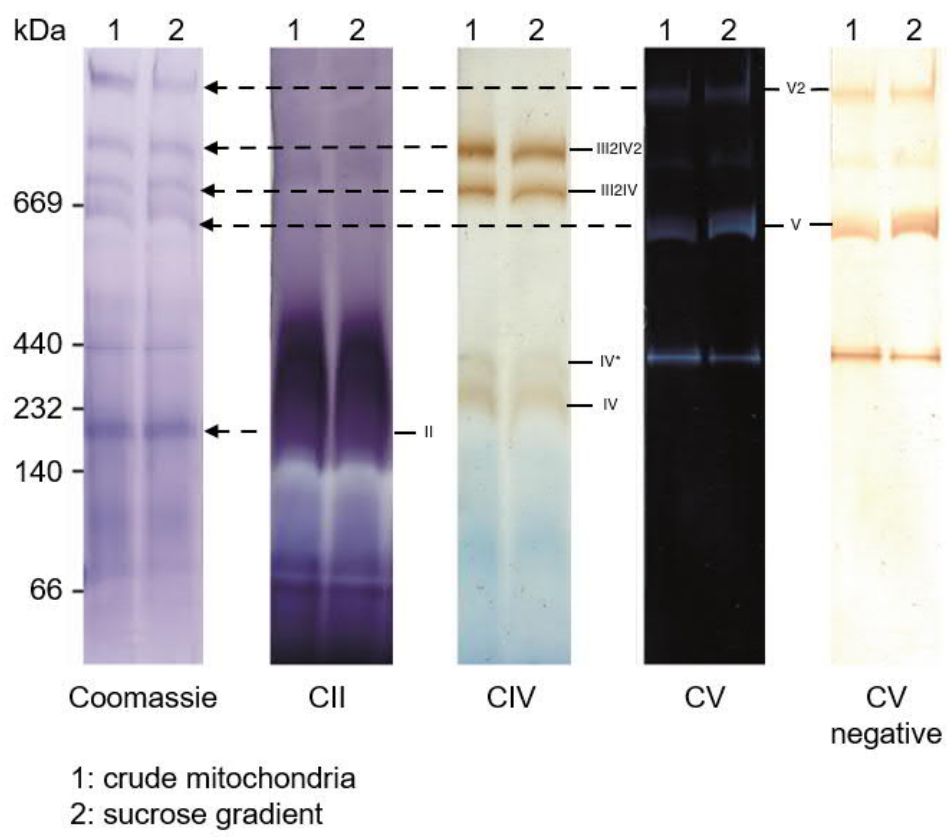

Figure 27: Activity staining of CII, CIV and CV after BN-PAGE. Frozen crude mitochondrial extract (lanes 1, crude mitochondria) and frozen purified mitochondria (lanes 2, sucrose gradient) were lysed with digitonin and subjected to BN-PAGE. Gel slices were treated with a combination of a substrate for the specific complexes and a redox-reactive, detectable dye. Coomassie, gel slice was Coomassie stained to show loading of equal protein amounts; CII, gel slice was treated with $\mathrm{NADH}$ as substrate and NBT as dye; CIV, gel slice was treated with reduced cytochrome $c$ and $\mathrm{DAB} ; \mathrm{CV}$, gel slice was treated with ATP and $\mathrm{Pb}\left(\mathrm{NO}_{3}\right)_{2} ; \mathrm{CV}$ negative, negative image of $\mathrm{CV}$ staining. II, CII; IV, CIV; V, CV; V2; CV dimer; III2IV(2), CIII-CIV supercomplexes. Experiments were performed and figure was prepared by Bettina Homberg, Cellular Biochemistry, University Medical Center Göttingen, PhD student in the GGNB program 'Molecular Medicine'. 


\subsubsection{Proteinase $\mathrm{K}$ assay to investigate mitochondrial membrane integrity}

For the cross-linking experiments with yeast mitochondria, a slightly hypotonic crosslinking buffer (20 mM HEPES pH 7.5, $100 \mathrm{mM} \mathrm{NaCl}$ ) was used, causing mitochondrial swelling. Slowly, mitochondria incorporate water, causing the outer membrane to burst, resulting in mitoplasts. The proteinase $K(P K)$ assay (see subsection 2.2.10) gives information about the membrane integrity of mitochondria, in this case especially of the inner membrane. $\mathrm{PK}$ is an unspecific protease digesting other proteins if it can access them. Outer and inner membrane of mitochondria function as natural barrier that cannot be penetrated by PK unless damaged. In this experiment, frozen purified mitochondria were incubated with cross-linking buffer containing two different concentrations of PK for

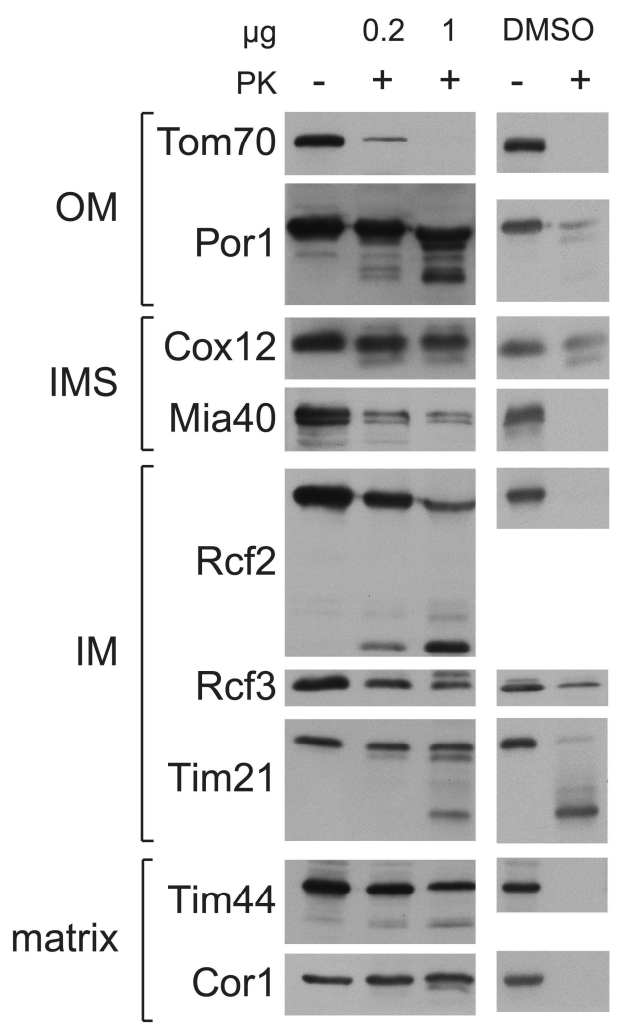

Figure 28: Proteinase $\mathrm{K}$ assay of frozen purified mitochondria. Mitochondria were treated with different concentrations of proteinase K (PK; "0.2+": $0.2 \mu \mathrm{g} \mathrm{PK} / \mu \mathrm{g}$ mitochondria; "1+": $1 \mu \mathrm{g} \mathrm{PK} / \mu \mathrm{g}$ mitochondria). After lysis, proteins were blotted. The Western blot was decorated against specific proteins of the mitochondrial subcompartments outer membrane (OM), intermembrane space (IMS), inner membrane (IM) and matrix. As controls, untreated mitochondria ("-") and mitochondria treated with $5 \%$ DMSO and sonication ("DMSO-" without PK, "DMSO+" with PK) were used. Bands that differentiate from the control in molecular weight or intensity indicate digestion products. Experiments were performed and figure was prepared by Bettina Homberg, Cellular Biochemistry, University Medical Center Göttingen, PhD student in the GGNB program 'Molecular Medicine'. 
$1 \mathrm{~h}$. After lysis, proteins were Western blotted and the blot decorated against proteins specific for the mitochondrial subcompartments outer membrane (OM), intermembrane space (IMS), inner membrane (IM) and matrix. The appearance of digestion products or vanishing signal intensities shed light on the membrane integrity of the mitochondria. As controls, untreated and mitochondria that were treated with $5 \%$ DMSO and by sonication were also investigated, i.e. PK can access proteins in all compartments. Tom70 and Por1 were representatively selected for the OM. Digestion products were detected in all PKtreated samples (Figure 28, lanes "0.2+", "1+" and "DMSO+"). Similar results were observed for IMS proteins Cox12 and Mia40 and for IM proteins Rcf2, Rcf3 and Tim21. Matrix proteins Tim44 and Cor1 remain unaffected by PK treatment and showed similar band patterns and intensities as the untreated control (lane "-"), indicating a fully intact inner mitochondrial membrane. Matrix proteins Tim44 and Cor1 were fully digested by PK in the control sample where mitochondria were treated with DMSO and by sonication (lane "DMSO+").

\subsection{Cross-linking combined with mass spectrometry of mitoplasts derived from human HEK293T cells}

The workflow that was established to cross-link mitochondria derived from yeast (see subsection 3.1.2) was applied to mitoplasts generated from mitochondria isolated from human HEK293T cells. Mitoplasts are mitochondria with the outer membrane stripped off, but the inner membrane being still intact. Mitoplasts instead of mitochondria were used for cross-linking to further reduce sample complexity and to identify more, even low abundant, interactions within the IM and the matrix. Additionally to the lysine-specific cross-linker BS3, 1-ethyl-3-(3-dimethylaminopropyl)carbodiimide hydrochloride (EDC) was used. EDC is a zero-length cross-linker and connects lysine residues to amino acids containing a carboxyl group, such as glutamate and aspartate. By using BS3 and EDC, not only lysinerich proteins will be addressed but also proteins with less lysine residues but more acidic amino acid residues. Human mitochondria were isolated from HEK293T cells (see subsection 2.2.12). Mitoplasts were generated and then cross-linked with BS3 or EDC (see subsection 2.2.14). Proteins were digested with trypsin, cross-linked peptides were enriched by peptide SEC (see subsection 2.3.5), and analyzed by LC-MS/MS. For data analysis, the successor software of pLink 1 was used, pLink $2^{258}$ (see subsection 2.4.2). This software is able to search cross-linked peptides against larger protein databases in a reasonable period of time. Therefore, all proteins that were identified based on their linear peptides (separately for the BS3- and the EDC-replicate) were included in the databases (1500 proteins for the BS3-data set and 1900 proteins for the EDC-data set). The results 
obtained by pLink 2 were not score-filtered but those identifications that were only supported by a single CSM were removed, similar to the yeast cross-linking data sets.

Culturing of HEK293T cells was maintained by Elena Lavdovskaia, Cellular Biochemistry, University Medical Center Göttingen, PhD student in the GGNB program 'Molecular Biology of Cells'. Isolation of human mitochondria, preparation of human mitoplasts and the cross-linking reactions were performed in collaboration with Elena Lavdovskaia.

\subsubsection{BS3 and EDC cross-link different regions of proteins}

In terms of total identified CSMs, cross-linked proteins and unique residue-to-residue cross-links, BS3 outperformed EDC in the unfiltered data set (Table 9). However, the relative number of interprotein cross-links in the filtered data set is higher for EDC. The overlap of cross-linked proteins of both data sets is $37 \%$. All identified cross-links are listed in Supplementary Table 5 provided on a CD-ROM attached to the hardcopy version of this thesis.

The low overlap suggests that BS3 and EDC cross-links different proteins. To address this question, the relative distribution of the isoelectric points $(\mathrm{pl})$ of all cross-linked proteins $^{298}$ were plotted (Figure 29). Although there is no significant difference, BS3 showed a slight tendency to cross-link proteins with higher pl values (pl 10-12), whereas EDC cross-linked proteins with slightly lower pl values (pl 8-10). The tendency is in accordance with the $\mathrm{pK}_{\mathrm{a}}$ values of side chains of lysine (10.67), aspartate (3.71), and glutamate (4.15), necessary for the BS3 and EDC reaction chemistries.

Table 9: Numbers of total identified cross-linked peptides spectrum matches (CSMs), cross-linked proteins, unique residue-to-residue cross-links and the interprotein cross-links in percent for the unfiltered and the filtered data set after BS3- or EDC-cross-linking of human mitoplasts. 'Single CSMs' in percent represent those unique residue-to-residue cross-links that were identified only by a single CSM.

\begin{tabular}{|c|c|c|c|c|}
\hline & $\begin{array}{c}\text { unfiltere } \\
\text { BS3 }\end{array}$ & EDC & $\begin{array}{l}\text { filtere } \\
\text { BS3 }\end{array}$ & EDC \\
\hline total CSMs & 10253 & 3478 & 9779 & 3069 \\
\hline cross-linked proteins & 433 & 280 & 284 & 191 \\
\hline $\begin{array}{l}\text { unique residue-to-residue } \\
\text { cross-links }\end{array}$ & 1284 & 907 & 854 & 508 \\
\hline interprotein cross-links & $12.7 \%$ & $12.5 \%$ & $7.0 \%$ & $10.8 \%$ \\
\hline single CSMs & $33.0 \%$ & $43.8 \%$ & & \\
\hline
\end{tabular}


The differences in cross-linking is also reflected by the very low overlap of peptideto-peptide cross-links (taking only into account the cross-linked peptides but not the actual cross-linked residues, because BS3 and EDC target different amino acids), which is only $2 \%$. These findings indicate that BS3 and EDC cross-link different regions of proteins and different proteins. Hence, cross-linking with these two cross-linkers deliver complementary results.

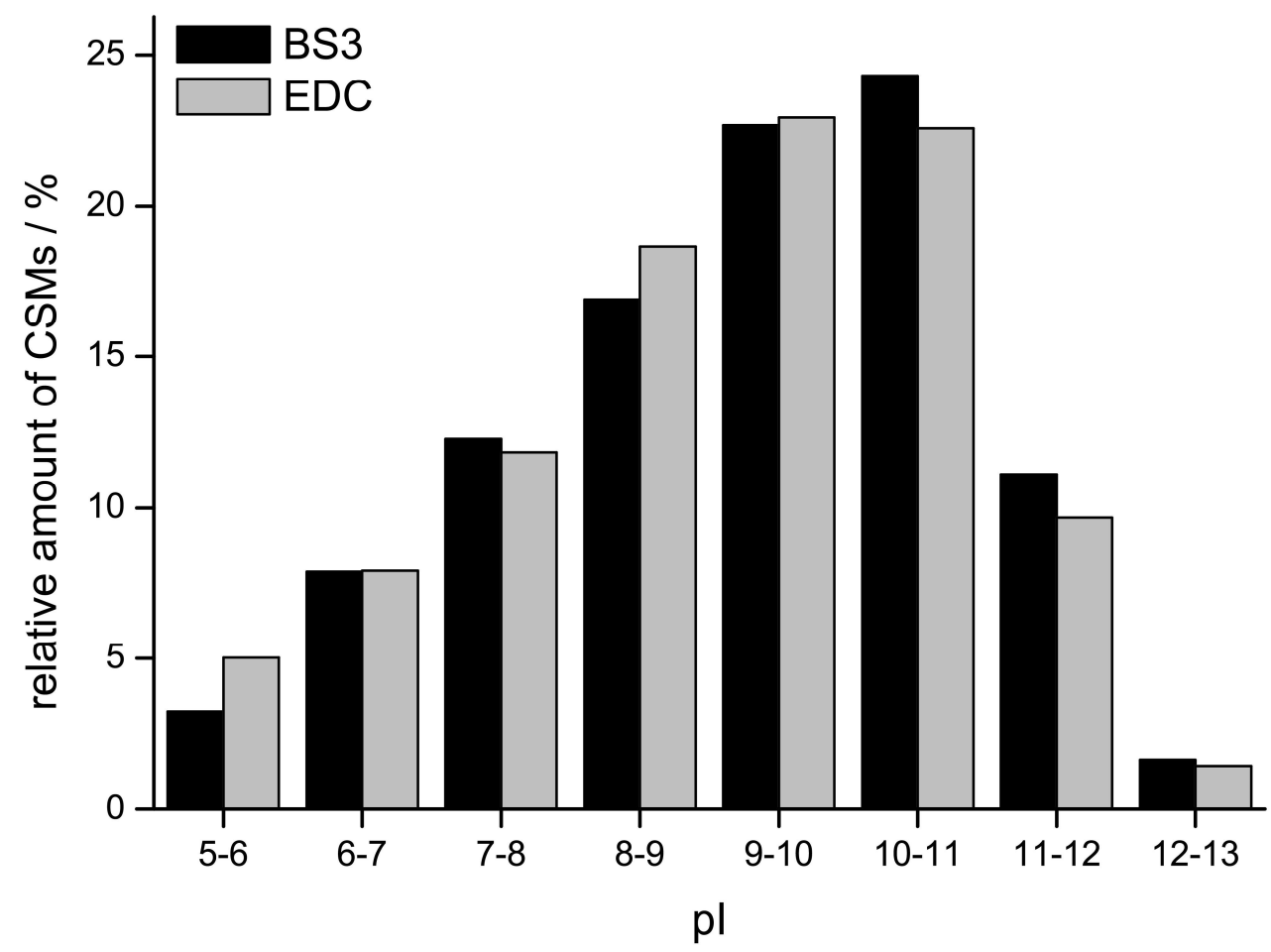

Figure 29: Relative distribution of isoelectric points ( $\mathrm{pl}$ ) of all proteins involved in cross-linking reactions with either BS3 (black bars) or EDC (light gray bars) in percent after cross-linking human mitoplasts.

\subsubsection{Intraprotein cross-links outnumber interprotein cross-links}

Irrespective of the used cross-linker, the majority of identified residue-to-residue crosslinks represented intraprotein cross-links, i.e. cross-links within one protein (93\% in the filtered BS3- and $89 \%$ in the filtered EDC-data set, Table 9). In both data sets, most intraprotein cross-links were identified within the chaperonin involved in mitochondrial protein import and macromolecular assembly (HSPD1 and HSPE1). In summary, these intraprotein cross-links account for $19 \%$ and $26 \%$ of all CSMs in the BS3- and EDC-data set, respectively (Table 10). Among the ten proteins that contributed the most to the numbers of spectra are e.g. ATP5A1 (BS3 and EDC), a subunit of the $F_{1} F_{0}$ ATP synthase, 
SSBP1 (BS3 and EDC), a single-stranded DNA-binding protein, DLD (BS3), the dihydrolipoyl dehydrogenase, and C1QBP (EDC), the complement component $1 \mathrm{Q}$ subcomponent-binding protein. Comparing these results to the abundance of proteins resulting from the identification search for linear peptides, seven out of these ten proteins per data set are among the 50 most abundant proteins per data set.

Table 10: Proteins involved in BS3- and EDC-induced cross-linking reactions ranked according to their numbers of cross-linked peptides spectrum matches (CSMs) contributing to all identified CSMs.

\begin{tabular}{r|lr|lr} 
Rank & Protein & $\begin{array}{r}\mid c \\
\text { relative } \\
\text { CSMs/ \% }\end{array}$ & Protein & $\begin{array}{r}\text { EDC } \\
\text { relative } \\
\text { CSMs/ \% }\end{array}$ \\
\hline $\mathbf{1}$ & HSPD1 & 13.22 & HSPD1 & 19.26 \\
$\mathbf{2}$ & HSPE1 & 5.41 & HSPE1 & 6.58 \\
$\mathbf{3}$ & DLD & 2.88 & CIQBP & 4.37 \\
$\mathbf{4}$ & ATP5A1 & 2.58 & ATP5A1 & 2.77 \\
$\mathbf{5}$ & DLAT & 2.40 & TUFM & 2.61 \\
$\mathbf{6}$ & SLC25A3 & 2.16 & MDH2 & 2.41 \\
$\mathbf{7}$ & TRAP1 & 2.00 & LITM1 & 2.35 \\
$\mathbf{8}$ & SSBP1 & 1.78 & AlFM1 & 2.31 \\
$\mathbf{9}$ & SLC25A5 & 1.73 & SSBP1 & 2.22 \\
$\mathbf{1 0}$ & HSPA9 & 1.65 & LRPPRC & 2.12
\end{tabular}

\subsubsection{Interprotein cross-links confirm ETC supercomplex formation}

Similar to the results of cross-linking yeast mitochondria, cross-linking of human mitoplasts also provided the majority of interprotein cross-links within the OXPHOS system and the mitoribosome (Figure 30). 79 unique protein-protein cross-links were identified in total. BS3-cross-linking contributed 40 of them and EDC-cross-linking 39, with an overlap of 5. No regularities in terms of proteins' functions or suborganellar localizations are noticeable when comparing BS3- and EDC-interprotein cross-links. The vast majority of interprotein cross-links is between proteins that are located either in the IM or in the matrix, indicating that the $\mathrm{OM}$ is indeed not present due to mitoplast generation. However, a few interactions were detected between OM- and IMS-proteins involving TOMM22, component of TOM (OM), VDAC2, the voltage-dependent anion channel (OM), GK, the glycerol kinase (OM), and AIFM1, the apoptosis-inducing factor 1 (IMS), and AK2, the adenylate kinase 2 (IMS). Also, cross-links between mitochondrial and non-mitochondrial proteins, such as histones and a DNA methyltransferase, were detected. This, in turn, might indicate impurities during 
mitochondria isolation. Since these proteins formed interactions to IM- and matrix proteins, a small portion of damaged mitochondria cannot be negated.

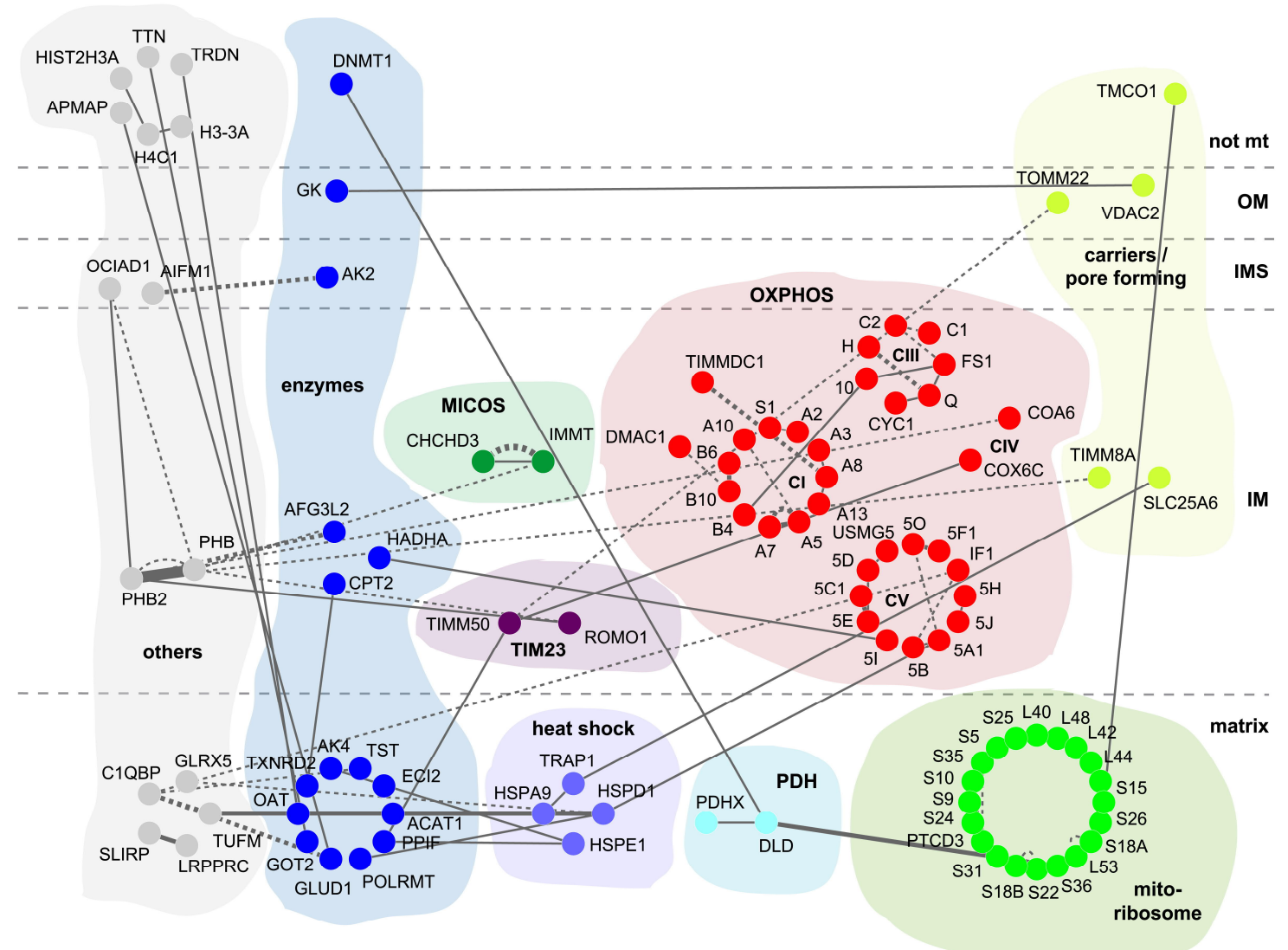

Figure 30: Global protein-protein cross-linking network in mitoplasts generated from human mitochondria. Only interprotein cross-links induced by BS3 (lines) and EDC (dashed lines) are illustrated. Thickness of the edges is proportional to the number of unique residue-to-residue crosslinks. Names of subunits of $\mathrm{Cl}, \mathrm{ClII}, \mathrm{CV}$ and the mitoribosome are abbreviated for the sake of readability (prefix $\mathrm{Cl}$, NDUF; prefix CIII, UQCR (except for CYC1); prefix CV, ATP, prefix mitoribosome, MRP). OM, outer membrane; IMS, intermembrane space; IM, inner membrane; not $\mathrm{mt}$, not mitochondrial; OXPHOS, oxidative phosphorylation system; PDH, pyruvate dehydrogenase complex; MICOS, mitochondrial contact-site and cristae-organizing system; TIM23, proteins that belong to the TIM23 complex; mitoribosome, mitochondrial ribosome. Networks visualized by Cytoscape ${ }^{269}$.

Remarkably, besides the chaperonin HSPD1/HSPE1 also other heat shock proteins like TRAP1 and HSPA9 were involved in cross-linking reactions. Especially by EDC, crosslinks within MICOS (IMMT to CHCHD3) and from MICOS to prohibitin (IMMT to PHB) were induced, indicating a very close proximity of the involved proteins. Prohibitin also formed cross-links to ROMO1, recently identified as constituent of the TIM23 complex ${ }^{299}$. Also, a cross-link between prohibitin and OCIAD1 was detected. In a former study ${ }^{300}$, OCIAD1 was already described as part of the prohibitin interactome. 
Within the OXPHOS system, subunits of $\mathrm{Cl}$, CIII and CV showed interprotein crosslinks. Differently from the yeast cross-linking data set, CII and CIV are barely covered. However, the cross-link between NDUFB4, subunit of $\mathrm{CI}$, and UQCR10, subunit of CIII, induced by BS3 confirms the formation of an ETC supercomplex in organello also in human mitoplasts. Here, residue K7 of NDUFB4 cross-linked to the $\mathrm{N}$-terminus of UQCR10 (Figure 31). The Ca-atoms of these residues are $11 \AA$ apart and fulfill the distance constraint of BS3 (30 ̊). Also, two factors that are involved in Cl assembly crosslinked to $\mathrm{Cl}$ subunits, namely DMAC1 to NDUFB10 and TIMMDC1 to NDUFA8, both induced by EDC. Remarkably, these two subunits of $\mathrm{Cl}$ are located at the periphery of the complex, underlining the validity of the identified cross-links.

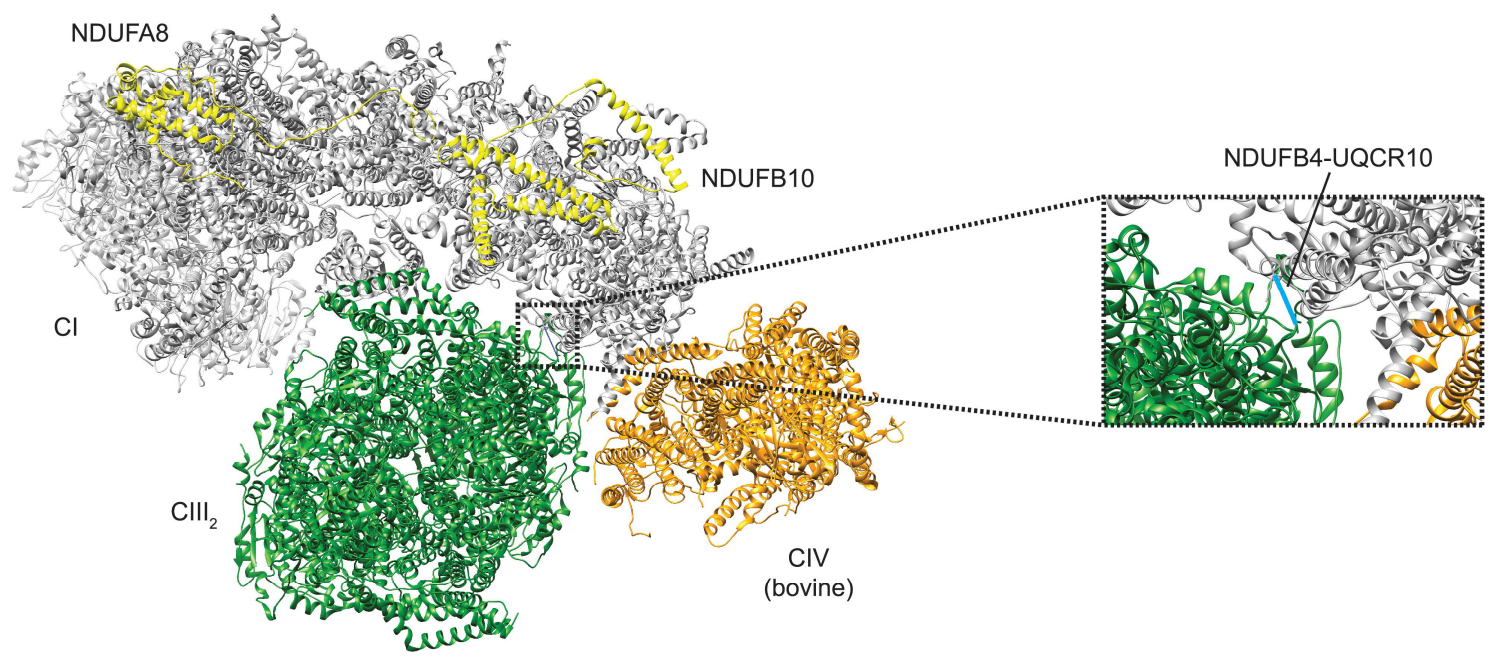

Figure 31: Human $\mathrm{CICll}_{2}$ supercomplex structure. ETC supercomplex structure including human $\mathrm{Cl}$ (gray), human $\mathrm{Clll}_{2}$ dimer (green) and bovine CIV (orange) (PDB: $5 \mathrm{XTH}^{90}$ ) visualized as top view from IMS. NDUFA8 and NDUFB10, subunits of $\mathrm{Cl}$, cross-linked to assembly factors and are highlighted in yellow. Inlet displays zoomed interface between $\mathrm{Cl}$ and CIII with the identified BS3cross-link between NDUFB4 and UQCR10 as blue bar $(11 \AA ̊$ ). 


\section{Discussion}

In this thesis, the differential protein-protein cross-linking networks in mitochondria derived from Saccharomyces cerevisiae grown on glycerol- or glucose-containing medium were investigated by chemical cross-linking in combination with mass spectrometry (XL-MS). Besides a qualitative approach, also a quantitative approach by using a stable-isotopically labeled cross-linker was performed to address this task. Additionally, mitochondria isolated from a $r o^{\circ}$ yeast strain lacking mitochondrial DNA (mtDNA) were cross-linked. It was sought to increase the analytical depth by using mitochondria from a strain that is not able to assemble a functional electron transport chain (ETC) and a mitochondrial ribosome. Subunits of these complexes are highly abundant in mitochondria. By reducing the complexity, other, lower abundant, complexes should be identifiable. Unfortunately, the obtained results from mitochondria isolated from the $r h o^{\circ}$ yeast strain did not reveal a significant higher number of cross-links in lower abundant mitochondrial protein complexes when compared to results obtained in mitochondria from wild type yeast grown on glycerolor glucose-containing medium. Therefore, the $r h o^{\circ}$ strain was mainly used as a 'disrupted' control system to validate the results gained from mitochondria isolated from yeast grown on glycerol- or glucose-containing medium. The optimized cross-linking workflow for yeast mitochondria was additionally applied to mitoplasts originating from human mitochondria of HEK293T cells. Here, two different cross-linkers, BS3 and EDC, which react with different amino acid residues were applied in order to identify additional protein-protein cross-links. In the following, the qualitative and quantitative XL-MS strategies will be discussed in detail. Furthermore, the identified protein-protein cross-links of selected proteins and protein complexes will be described against the background of their biological context.

\subsection{Integrity of mitochondrial membranes and its impact on structural analysis}

In this thesis, mitochondria were used that have been purified by tandem sucrose gradient centrifugation after isolation. This was done to reduce impurities that co-isolate with the mitochondria, such as mitochondria-associated membranes (MAMs) like the endoplasmic reticulum (ER) and its proteins. Furthermore, mitochondria have been frozen at $-80^{\circ} \mathrm{C}$ prior to cross-linking. The purification is a well-established procedure to gain highly pure mitochondria ${ }^{239}$ that are also functional, at least in regard to protein import ${ }^{301}$. In this thesis, this has been confirmed by quality control experiments (see section 3.5). In general, the isolated and purified mitochondria were functional with respect to respiration, protein import, oxidative phosphorylation (OXPHOS) complex activities and inner membrane 
integrity. However, especially via the respirometry assay it could be demonstrated that purification and freezing have a negative effect on mitochondrial performance (see subsection 3.5.1). Already in 1971, Packer et al. ${ }^{302}$ have shown that high concentrations of sucrose (above $0.4 \mathrm{M}$ ), an osmotically active compound, might dehydrate mitochondria derived from rat liver, therefore inducing unspecific changes in lipid compositions of mitochondrial membranes, unusual aggregation of protein complexes and inhibited respiration. The tandem sucrose gradient centrifugation that purifies the mitochondria might therefore have a negative effect also on respiration in yeast mitochondria. However, these effects can be reversed by incubation of mitochondria in hypotonic solutions. Since this thesis does not only rely on pure mitochondria but also on unaltered structural arrangements of protein complexes, identified protein-protein cross-links were carefully curated with respect to biological plausibility to avoid any artefacts. Nonetheless, a small amount of slightly damaged mitochondria used for cross-linking cannot be negated.

\subsection{BS3 and its ability to cross-link proteins of all mitochondrial subcompartments}

Two commonly used and commercially available cross-linkers are disuccinimidyl suberate (DSS) and its sulfonated derivative bis(sulfosuccinimidyl) suberate (BS3) ${ }^{183}$. Both of them are non-cleavable, homobifunctional $\mathrm{N}$-hydroxysuccinimide (NHS) esters that preferably react with the $\varepsilon$-amino group of lysine residues and protein $\mathrm{N}$-termini. Both cross-linkers possess a spacer arm that, after reaction, covers a distance of $11.4 \AA$. The difference between both compounds is the charge in BS3 that is introduced by the sulfo-group. Therefore, BS3 is charged, hydrophilic, and, hence, water-soluble. DSS is uncharged, hydrophobic, and therefore only soluble in organic solvents. Consequently, DSS is supposed to be membrane-permeable, while BS3 is not. When cross-linking mitochondria, an organelle with two lipid bilayers, membrane-permeability of the cross-linking ingredient is a key parameter. The functionality of DSS for cross-linking human mitochondria was demonstrated recently ${ }^{101}$. However, the use of DSS comes along with a major drawback. Since organic compounds such as DMSO are necessary for the solubilization of DSS (or, in general, for all membrane-permeable cross-linkers), an interference of such solvents with the membrane system has to be considered, at least when using higher concentrations of the cross-linker. In general, DMSO tends to induce water pores in membranes and increases their permeability ${ }^{303}$. By applying transmission electron microscopy, Yuan et al. ${ }^{276}$ could show that mitochondria in cultured astrocytes treated with $1 \%$ DMSO within a time period of $24 \mathrm{~h}$ underwent swelling. An increase to $5 \%$ DMSO resulted in even more severe damages such as disruption and loss of cristae. While 0.5- 
$1.5 \%$ DMSO are widely accepted especially in cell cultures addressing medical problems ${ }^{304}$, higher concentrations seem to be problematic for membrane integrity. To catch also low abundant proteins and their interactions, a high cross-linker concentration is crucial ${ }^{211}$. Therefore, using a water-soluble cross-linker for sensitive membrane systems might be the better choice. Based on this information, BS3 was tested in this study for its suitability to cross-link mitochondria, although it is supposed to be membraneimpermeable.

Surprisingly, BS3 showed similar cross-linking efficiency as DSS (with $1 \%$ DMSO) in all mitochondrial subcompartments (see subsection 3.1.1). This raises the question, how a negatively charged $570 \mathrm{Da}$ molecule can pass through two functional membrane bilayers. The outer mitochondrial membrane might act as a sieve with free permeability for molecules smaller than 1-2 kDa due to Por $1^{305}$. Por1, also known as the voltagedependent anion channel (VDAC), is a highly abundant protein within the outer mitochondrial membrane, forming a pore with a diameter of approximately $2-3 \mathrm{~nm}$. In its open form this channel is selective to anions ${ }^{306}$. More recent studies suggested a permeability of Por 1 for hydrophilic small molecules of up to $5 \mathrm{kDa}^{307}$ (also for ATP as modeled for murine $\mathrm{VDAC}^{308}$ ) and even linear DNA might use Por1 as transporter in plants ${ }^{309}$. Therefore, Por 1 might be one entry gate to pass through the outer mitochondrial membrane. Additionally, besides the translocase of outer membrane (TOM) complex that is responsible for protein import into mitochondria, three other outer membrane transporters have been identified recently, namely Ayr1, Omc7 and Omc8 ${ }^{310}$. Ayr1 is a NADPH-dependent channel whereas the latter two are anion channels whose substrates are still unknown. In theory, also these channels might aid BS3 import.

The inner mitochondrial membrane forms a closed system with specific carrier proteins what makes it much more difficult for the charged BS3 molecule to pass through this particular membrane. One of the most abundant carrier proteins is Pet9, the ADP/ATP exchange carrier ${ }^{311}$. Since this protein carries ADP and ATP, which are both charged and hydrophilic molecules, Pet9 might aid the transport of BS3 in an unspecific manner. In the past, it was assumed that Pet9 is the pore forming unit of the mitochondrial megachannel (MMC), also known as permeability transition pore (PTP) $)^{312,313}$. More recent studies propose that rather the $F_{1} F_{0}$ ATP synthase than Pet9 is the pore forming unit ${ }^{314,315}$. The PTP is $\mathrm{Ca}^{2+-}$ and reactive oxygen species (ROS)-dependent and forms, similar to Por 1 , a $3 \mathrm{~nm}$ large pore, allowing for molecules up to $1.5 \mathrm{kDa}$ to pass ${ }^{316-318}$. The PTP is also responsible for mitochondrial swelling ${ }^{319}$ that might have been induced by the slightly hypotonic cross-linking buffer used in this thesis. However, channels and pores might also become inactive after reacting with the cross-linker. Therefore, it might also be possible that mitochondrial membrane integrity was slightly altered due to sucrose purification and 
freezing. Additionally, the cross-linking reaction was performed for $1 \mathrm{~h}$ at room temperature in a slightly hypotonic buffer that might have increased the damage enabling the cross-linker to reach every mitochondrial subcompartment. The membrane integrity of mitochondria could be tested, for example, by recording electron microscopy (EM) images of mitochondria after incubation in cross-linking buffer with and without cross-linker.

\subsection{Benefits and drawbacks of restricted databases for cross-linked peptides searches}

Cross-linked peptides were identified by database search with the software pLink $1^{255,256}$. It identifies peptides cross-linked with a non-cleavable cross-linker such as BS3 and DSS, i.e. the covalent bond formed between two amino acids will not be cleaved during the fragmentation in the gas phase of the mass spectrometer (see section 4.8). This poses a huge computational challenge, since one spectrum does not only contain information of fragment ions of one peptide - as is the case in common proteomics - but information of fragment ions of two peptides, linked by the cross-linker. Consequently, spectra displaying cross-links are chimeric and, hence, more complex. A major bottleneck in cross-linking experiments is therefore the computational analysis of cross-linked peptides spectra ${ }^{180}$. In contrast to a conventional database search of linear peptides, the search space of possible peptide combinations increases quadratically (in fact: $\left(n^{2}+n\right) / 2$, with $n$ being the number of tryptic peptides). This is termed the $n^{2}$-problem ${ }^{180,220}$ and makes the analysis computationally expensive. In XL-MS studies that investigate single proteins or protein complexes containing only a few proteins, the $n^{2}$-problem in database search can be neglected ${ }^{180}$. However, the $n^{2}$-problem becomes unsurmountable in the elucidation of large-scale protein-protein interactions. The analysis of 50 cross-linked proteins inflates the search space as much as a search for linear peptides of the whole human proteome ${ }^{220}$. The higher the number of proteins within the database, the higher the possibility of identifying false positives. To counteract this, in this thesis restricted databases were generated for the identification of cross-linked peptides, reducing the search space to the 400 most abundant proteins (proteins that are included in the respective databases are listed in Supplementary Table 6 provided on a CD-ROM attached to the hardcopy version of this thesis). Three benefits come along with the use of restricted databases: (i) Database searches with pLink 1 were still possible in a reasonable time frame. (ii) Smaller databases decrease the number of false positive identifications ${ }^{195}$. (iii) Cross-linking reactions occur mostly within and between high abundant proteins ${ }^{211,320}$ that are covered by the used databases in this thesis. Interactions between low abundant proteins are therefore anyway difficult to detect. A similar observation was made by Liu et al. ${ }^{100}$ who cross-linked murine 
4.4 Protein-protein cross-links in mitochondria derived from yeast grown on either glycerol- or glucose-containing medium

mitochondria. Although the authors used an MS-cleavable cross-linker (DSSO) and searched against the whole murine mitochondrial proteome (see subsection 4.8.2), approximately $90 \%$ of all unique residue-to-residue cross-links were identified between the approximately 500 most abundant proteins. The main drawback of using restricted databases is that cross-links within and between low abundant proteins or their cross-links to high abundant proteins will not be detected because these proteins are not included in the databases. Especially in this thesis, in which the interactomes of mitochondria derived from yeast grown on different carbon sources have been investigated, the selection of only the 400 most abundant proteins bears limitations. Since the metabolism of yeast cells is affected by the carbon source ${ }^{294,295}$, different genes will be differentially expressed leading to different abundances of proteins ${ }^{72}$. The glucose-induced repression of genes encoding proteins participating in e.g. oxidative phosphorylation or the TCA cycle f5,76,321 $^{7 t r o n g l y}$ influences the protein abundances in the respective samples. Therefore, differences in the cross-linking pattern of mitochondria derived from yeast grown on either glycerol- or glucose-containing medium mainly reflect the different abundances of the translated proteins under both conditions. Furthermore, proteins cross-linked under one condition might not be identified under the other condition because two separate databases with the 400 most abundant proteins were used for searching. Therefore, for the comparison of the cross-linking results of the glucose and glycerol data sets shown in subsection 3.1.3, Figure 8, only those cross-links of proteins present in both databases were considered. In this regard, the quantitative XL-MS approach and the subsequent database search is more robust, because the samples are pooled and, hence, only one database with the 400 most abundant proteins identified under both conditions was used (see section 4.6). Other strategies for search space reduction are discussed in subsection 4.8.2.

\subsection{Protein-protein cross-links in mitochondria derived from yeast grown on either glycerol- or glucose-containing medium}

The following paragraphs will discuss the cross-linking results obtained from mitochondria derived from yeast grown on either glycerol- or glucose-containing medium in the context of interactions, structural assemblies and functions of proteins.

\subsubsection{A general comment on the filtering of the XL-MS data sets}

Two biological cross-linking replicates with two technical LC-MS/MS measurement replicates each were performed for each yeast growth condition. Results gained from each of the replicates were condition-specifically merged. In a different strategy, only the overlap 
of cross-links in the replicates might have been considered, which increases the reliability of the data but due to the stochastic nature of data-dependent acquisition ${ }^{163}$ in XL-MS a lot of true positive cross-links would have been missed in this case. Therefore, the latter strategy was not applied. To increase the reliability of the data, i.e. filtering out putative false positive interactions or artefacts, residue-to-residue cross-links that were supported by only a single cross-linked peptides spectrum match (CSM) and had a pLink 1-specific spectrum score below 4 (- $\log _{10}$-transformed) were excluded from further analysis. The rationale for excluding single CSMs is based on the assumption that interactions supported by just a single CSM within $132 \mathrm{~h}$ of measurement time per condition are not reproducible. The rationale for excluding CSMs with a score below 4 is based on experience gained in studies in which purified complexes were cross-linked and subsequently mapped on crystal structures as happened for example in Vos et al. ${ }^{322}$, where all CSMs were evaluated manually. A CSM was rated as "good" if at least three consecutive fragment ions of both peptides were annotated. This was frequently observed for CSMs with a score higher than 4. To avoid manual inspection of CSMs of the mitochondrial samples, the established filtering steps were applied. However, it cannot be excluded that a minor part of false positives is still present in the data sets and that a minor part of true positives was filtered out. In the end, the filtering steps increased the overlap of residue-to-residue cross-links between the biological replicates from $39 \%$ to $55 \%$ in the glycerol and from $45 \%$ to $62 \%$ in the glucose data set (see Supplementary Figure 1). Of note, cross-linking results obtained from pLink 1 searches are filtered according to a false discovery rate (FDR) of $1 \%$ on spectrum level by default. The above outlined filtering steps in combination with the application of $1 \%$ FDR applied on all CSMs are stringent criteria. Therefore, the crosslinks in this study can be considered as reliable.

\subsubsection{Majority of cross-linked proteins localized to the inner membrane or matrix}

Protein localization assignments in this thesis are based on a study performed by Vögtle et al. ${ }^{142}$ With a comprehensive MS-based approach including SILAC they could confidentially assign 818 mitochondrial yeast proteins to one of the four sublocalizations outer membrane (OM), intermembrane space (IMS), inner membrane (IM) and matrix. Most of the assigned proteins, $82 \%$, belong to either the IM or the matrix indicating the most crowded sublocalizations. This was also confirmed by Morgenstern et al. ${ }^{72}$ and is also reflected in this thesis. Above $80 \%$ of all identified protein-protein cross-links are between proteins belonging to either the IM or the matrix irrespective of the used carbon source (see subsection 3.1.3). This is reasonable since the IM and the matrix harbor 
4.4 Protein-protein cross-links in mitochondria derived from yeast grown on either glycerol- or glucose-containing medium

abundant protein complexes that belong to the OXPHOS system or to metabolic pathways such as the TCA ${ }^{21,323}$. Strikingly, interactions between OM- and IMS-proteins, IM- and IMSproteins or OM- and IM-proteins were barely identified in both conditions (1.5\% of all identified protein-protein cross-links per condition after filtering, see subsection 3.1.3, Figure 7). This could have been caused using the slightly hypotonic cross-linking buffer that might have induced mitoplast formation. Hypotonic solutions induce swelling and the $\mathrm{OM}$ is stripped off ${ }^{324}$. While this increases the spatial distance between OM- and IMproteins, it also affects IMS-proteins. IMS-proteins will then be released and are no longer in their natural environment, and, hence, no longer in cross-linkable distance to OM- or IM-proteins.

In general, in this thesis 261 and 260 cross-linked proteins could be identified in the glycerol or the glucose data set, respectively. The unfiltered data represent 315 and 313 proteins, respectively. This is less than a third of the estimated mitochondrial yeast proteome ( 1000 proteins) and less cross-link identifications than in other studies (see subsection 4.4.8, Table 11). Besides specificities within the data analysis workflow that are discussed in subsection 4.4.8, also biochemical factors can influence the number of identifications. As already mentioned in section 4.3, the protein abundances and, hence, the restricted databases used in this thesis have a major effect. Furthermore, lysine residues of proteins must provide a certain level of solvent accessibility so that a reaction with the cross-linker can actually occur. Transmembrane regions of proteins (e.g. in the $\mathrm{OM}$ and IM) usually barely contain lysine residues ${ }^{325}$. Their identification, if at all, results from cross-linking events within their soluble regions. In addition to that, membrane proteins are difficult to solubilize ${ }^{326}$. Although SDS was used as a lysis detergent, it is not guaranteed that every membrane protein was solubilized. Also, proteins were subsequently precipitated and resuspended. In that process some proteins might also get lost. Especially cross-linked proteins are difficult to resuspend after precipitation (data not shown). Finally, since the cross-linker primarily reacts with lysine residues, these lysine residues cannot be recognized by proteases such as trypsin, effecting the digestion efficiency. Cross-linked peptides are therefore longer than linear ones and some combinations might be unfavorable for sufficient ionization in the ion source of the mass spectrometer. An optimized lysis protocol or the application of several proteases (sequential digestion, see 4.4.8) in combination with other cross-linkers might increase the identification of cross-linked peptides ${ }^{327}$. 


\subsubsection{Differences in the protein-protein cross-linking patterns between mitochondria derived from yeast grown on either glycerol- or glucose- containing medium}

The major goal of this thesis was to elucidate differences in the protein-protein crosslinking patterns between purified mitochondria derived from yeast grown on either glycerolor glucose-containing medium. While 74 unique protein-protein cross-links are common between both conditions, e.g. within the OXPHOS system, in the mitochondrial ribosome, the PDH or the TCA cycle (see Supplementary Figure 3), indicating the assembly of important complexes in both conditions, differences between both conditions could also be identified. Proteins that were uniquely identified in one of the two conditions were subjected to pathway enrichment analysis (see subsection 3.1.3). In the glycerol condition, pathways like oxidative phosphorylation, metabolic pathways and TCA cycle were enriched, while in the glucose condition pathways like amino acid biosynthesis and coenzyme $Q$ biosynthesis were enriched. This is in line with the performed proteomic analysis in this thesis (see section 3.4) and also with other studies that performed deep quantitative analysis of the mitochondrial yeast proteome of different conditions ${ }^{72,148}$. The comparable results on protein level between proteomic studies and the cross-linking study presented in this thesis indicate that the differences in protein-protein cross-linking patterns between both conditions strongly correlate with the protein abundances per se (see section 4.3). These differences in protein abundances can be explained by the different energy and metabolic demands of the yeast cells. Yeast grown on nonfermentable carbon sources such as glycerol satisfy their energy demand by producing ATP via the OXPHOS system in mitochondria, while yeast grown on fermentable carbon sources such as glucose mainly produce energy by fermentation ${ }^{74,79}$. Glucose is a known repressor of several genes encoding proteins responsible for glycerol metabolism ${ }^{328}$ or respiratory enzymes ${ }^{329}$. Approximately $15 \%$ of all mitochondrial proteins are linked to respiratory processes under non-fermentable conditions ${ }^{21}$. Strikingly, these $15 \%$ sum up to more than $50 \%$ of the mitochondrial protein mass ${ }^{21}$. This is in compliance with proteomic studies that focused on changes of protein abundances during the diauxic shift $^{10,145}$. The diauxic shift describes the transition of yeast cells from fermentative to respiratory metabolism coming along with a significant increase of abundances of proteins belonging to the OXPHOS system or the TCA cycle. These findings explain why proteins belonging to the OXPHOS system or other metabolic pathways are more abundant under non-fermentable conditions and, hence, are more prone to cross-linking reactions. 
4.4 Protein-protein cross-links in mitochondria derived from yeast grown on either glycerol- or glucose-containing medium

\subsubsection{The oxidative phosphorylation system}

\section{ETC supercomplex formation}

In this thesis, the formation of supercomplexes within the ETC in yeast cells could be demonstrated by identifying cross-links between CIII and CIV. Regardless of the carbon source, connections between Qcr6, a subunit of CIII, and both Cox5a and Cox9, subunits of CIV, could be identified (see subsection 3.1.9). This indicates that the mentioned subunits of both complexes must be in close proximity to each other and supports the "solid state" model of supercomplex arrangements ${ }^{103}$. Back in the year 2000, Schägger and Pfeiffer could already provide evidence for the existence of arrangements between complexes of the OXPHOS system ${ }^{87}$. Previously, it was assumed that these complexes were arranged randomly within the IM. Although interactions between $\mathrm{Cl}$ and $\mathrm{CIII}^{330}$ and between $\mathrm{Cll}$ and $\mathrm{CIII}^{331}$ in mammalian mitochondria were reported decades ago, bile salts that can induce protein aggregation were used for complex isolation in these studies. Schägger and Pfeiffer, in turn, mildly solubilized membrane proteins derived from yeast and mammalian mitochondria with digitonin and subjected them to blue native (BN)-PAGE. With this approach, they could identify a $\mathrm{CIII}_{2} \mathrm{CIV}_{1}$ and a $\mathrm{CIII}_{2} \mathrm{CIV}_{2}$ supercomplex with CIV quantitatively attached to CIII in yeast and termed it the 'respirasome'. In the same year, Cruciat et al. ${ }^{88}$ could confirm these results with a similar approach. The abundance of these two supercomplexes is dependent on the used carbon source for reasons discussed in subsection 4.4.3. Non-fermentable carbon sources shift supercomplex formation to the $\mathrm{CIII}_{2} \mathrm{CIV}_{2}$ stoichiometry. However, the participation of other complexes was not detected with this approach. Even with a mild solubilization by digitonin it cannot be excluded that (especially weak and transient) interactions might be disturbed before analysis. AcínPérez et al. ${ }^{89}$ finally provided evidence for supercomplex formation by functional studies. The authors isolated intact supercomplexes from murine mitochondria containing $\mathrm{Cl}, \mathrm{Cll}$, CIII, and CIV after BN-PAGE separation and demonstrated oxygen consumption in a respirometry assay.

One benefit of XL-MS is the identification of interactions between proteins in their native environment, i.e. in organello. XL-MS studies performed by Schweppe et al. ${ }^{99}$ and Liu et al. ${ }^{100}$ could already provide evidence for ETC supercomplex formation in murine mitochondria in organello. Cross-links between $\mathrm{Cl}$ and CIII (Schweppe et al.) and even between all OXPHOS complexes CI-CV (Liu et al.) could be identified corroborating the existence of ETC supercomplexes under native conditions. Further evidence for supercomplexes were provided by pseudo-atomic cryo-EM structures of $\mathrm{ClII}_{2} \mathrm{CIV}_{(2)}$ arrangements in Saccharomyces cerevisiae ${ }^{91,93,332}$ and $\mathrm{Cl}_{1} \mathrm{ClI}_{2} \mathrm{CIV}_{1}$ arrangements in mammals, namely Sus scrofa ${ }^{92}$ and Homo sapiens ${ }^{90} .90 \%$ of the cross-links identified in 
this thesis mapped onto the $\mathrm{CIII}_{2} \mathrm{CIV}_{2}$ supercomplex structure published by Hartley et al. ${ }^{91}$ satisfied the distance constraint given by the cross-linker BS3 (30 $)$. Amongst them are the described cross-links between the subunits of CIII and CIV validating the results presented in this thesis. However, based on these data, a differentiation between the two stoichiometries $\mathrm{CIII}_{2} \mathrm{CIV}_{1}$ and $\mathrm{CIII}_{2} \mathrm{CIV}_{2}$ observed by Schägger and Pfeiffer ${ }^{87}$ is not possible due to the symmetry of the complex. While the higher number of cross-links within the ETC in mitochondria isolated from yeast grown on glycerol-containing medium correlates with the higher protein abundances compared to the glucose condition, the higher number of cross-links also supports the "plasticity" model of the ETC complex organization ${ }^{103}$ : under non-fermentable conditions, respiration has to work on high capacity, leading to supercomplex formation, while under fermentable conditions, ETC complexes might freely diffuse through the IM since respiration is limited.

\section{Ndi1 as part of an ETC supercomplex}

Not only cross-links between CIII and CIV were observed in this study, but all complexes of the OXPHOS system were cross-linked in both conditions (see subsection 3.1.5, Figure 10). The number of identified cross-links within these complexes was twice as high under glycerol than under glucose condition. This is due to the higher demand of yeast cells growing on non-fermentable carbon sources such as glycerol to produce energy via respiration and the resulting abundance increase of proteins involved in respiratory and metabolic pathways (see subsection 4.4.3).

Remarkably, also Ndi1, the internal NADH:ubiquinone oxidoreductase on matrixside, cross-linked to both Qcr2 and Qcr7, subunits of CIII, in mitochondria from yeast grown on glycerol-containing medium. Under glucose condition, Ndi1 cross-linked to Qcr2. Yeast mitochondria lack a proton pumping $\mathrm{Cl}$. Redox reactions of $\mathrm{NADH}$ and ubiquinone are therefore performed by Ndi1 (and the external reductases Nde1 and Nde2 located in the IMS). Based on the cross-links, an $\mathrm{Ndi}_{1} \mathrm{Cll}_{2} \mathrm{CIV}_{2}$ supercomplex structure was proposed (see subsection 3.1.9, Figure 16). For this, the two unique cross-links identified in the glycerol data set were used for docking experiments. Steric hindrance between Ndi1 and CIV was excluded by calculating the accessible interaction space (see subsection 3.1.9, Figure 15). Biochemical approaches such as BN-PAGE and clear native (cn)-PAGE in combination with activity staining of $\mathrm{NADH}$ dehydrogenases and subsequent protein identification by MS could demonstrate that Ndi1 is part of higher molecular weight respiratory supercomplexes ${ }^{83,333,334}$. However, proofs of a direct interaction between Ndi1 and CIII and their structural arrangement were missing so far. Three arguments support the proposed interaction between Ndi1 and CIII: (i) Assuming that the interaction between Ndi1 and CIII is weak or at least weaker than the interaction between CIII and CIV, the 
4.4 Protein-protein cross-links in mitochondria derived from yeast grown on either glycerol- or glucose-containing medium

direct interaction between Ndi1 and CIII might have escaped detection so far $^{333}$. Commonly used approaches to study protein-protein interactions like immuno- or affinitypurifications usually use detergents such as SDS, Triton X-100, DDM or mild detergents like digitonin for solubilization. Although tethered to the IM with its C-terminal membrane anchor, Ndi1 is a soluble matrix protein. CIII, in turn, is a bulky transmembrane complex. The interaction between Ndi1 and CIII can only occur on matrix-side with a very small interaction interface, much smaller than the one between CIII and CIV (CIV is also a transmembrane complex). (ii) The same protein-protein cross-links (Ndi1 to Qcr2 and Ndi1 to Qcr7) were identified in a recent XL-MS study on yeast mitochondria performed by Makepeace et al. ${ }^{102}$. (iii) There is a biological advantage of supercomplex formation. Although still under discussion, most likely it supports substrate channeling accelerating the electron transfer cascade ${ }^{85,87,89,335,336}$. Since the participation of $\mathrm{Cl}$ in an ETC supercomplex is already confirmed in mammals, it is also reasonable that Ndi1 performing the same redox reaction as $\mathrm{Cl}$ in mammals - participates in an ETC supercomplex in yeast. Since CIII forms a dimer in the supercomplex structure, even an $\mathrm{Ndi}_{2} \mathrm{CIII}_{2} \mathrm{ClV}_{2}$ higher-order supercomplex structure is conceivable by docking Ndi1 on both sides of $\mathrm{Clll}_{2}$.

\section{Pet9 cross-links to ETC complexes}

Pet9, also known as Aac2, is the major ADP/ATP carrier within the $1 \mathrm{IM}^{337-339}$. With more than $10^{5}$ copies per cell it is one of the most abundant proteins in yeast ${ }^{72}$. In the glycerol data set, cross-links of Pet9 to all ETC complexes were detected (see subsection 3.1.5, Figure 10). This suggests that Pet9 is in close proximity to the ETC. Being in close proximity to the ETC indeed is biologically meaningful, since Pet9's function is to transport $A D P$ as substrate for $C V$ from the cytosol to the matrix and ATP as product of $C V$ the other way round ${ }^{311,340}$. Dienhart et al. ${ }^{97}$ and Claypool et al. ${ }^{98}$ independently of each other could provide biochemical evidence for an association of Pet9 to the CIII-CIV supercomplex in yeast. Cells lacking Pet9 are not able to grow on non-fermentable carbon sources due to an impaired OXPHOS system ${ }^{341}$. Especially, the functionality of CIV is hampered ${ }^{97,98}$. While Claypool et al. ${ }^{98}$ claimed with caution that the physical association increases activities of both Pet9 and CIV, a few years later, the same group could demonstrate that Pet9's function as transporter and, hence, its impact on a functional protein translation from mitochondrial DNA per se is responsible for CIV's activity ${ }^{342}$. Pet9 also cross-linked to $\mathrm{Cll}$ and Ndi1. Sdh2, a subunit of Cll, could already be identified as potential interactor of Pet9 by Claypool et al. ${ }^{98}$ The direct interaction between Pet9 and Ndi1 as shown by cross-links in this thesis is undescribed so far. Pet9 participates in a local 'structural hub' 
consisting of CII, CIII, CIV, and Ndi1, indicating even more complex supramolecular structures in yeast mitochondria.

\section{Min8 is associated with the cytochrome $c$ oxidase}

XL-MS is suitable to identify even weak and transient interactions due to its ability to covalently connect proteins that are in close proximity. It also aids the identification of so far unknown interactions (e.g. Pet9 and Ndi1, see above; further examples are described in subsection 4.4.6). Furthermore, by identifying interactions of uncharacterized proteins, a biological classification of them might be deduced. In this thesis, this was successfully put into practice with Min8. Min8 cross-linked to Cox12, a subunit of CIV, supported by two unique residue-to-residue cross-links, and to Pet9, whose association with the ETC was discussed above. Makepeace et al. ${ }^{102}$ could also identify cross-links between Min8 and Cox12 as well as Pet9. Min8 is a so far uncharacterized protein of $8 \mathrm{kDa}$ encoded by the open reading frame (ORF) YPR010C-A and conserved among yeast. Morgenstern et al. ${ }^{72}$ could localize Min8 to the IM and could also define its orientation (see subsection 3.1.10). While its transmembrane region is located within the IM, its C-terminal part extends into the IMS and its N-terminal part into the matrix. These information are reflected by Min8's structure that was modeled ab initio. Based on the cross-links, it is suggested that Min8 is associated with the ETC, in fact with CIV. The calculated structure was docked to the CIV structure based on the identified cross-links and physicochemical properties. Pet9 and its cross-links to Min8 and Cox9, a subunit of CIV, were included to provide a second spatial constraint for the docking of Min8. Unfortunately, all cross-links between Min8 and Cox12 occurred on IMS-side. No cross-links were identified on matrix-side between Min8 and CIV, but between Min8 and Pet9. Since Pet9 cross-linked to CIV on matrix-side, the triangular connection Min8-CIV-Pet9 was used in the docking experiment mainly to fix the position of Min8 on matrix-side (see subsection 3.1.10, Figure 17).

To further consolidate the exact position of Min8 within CIV, oxidative cross-linking was applied (see subsection 3.1.10, Figure 18). Cu(II) ions can induce disulfide bridge formation between cysteine residues located in close distance to each other ${ }^{281}$. In contrast to lysine residues, cysteine residues are also part of transmembrane regions as is the case for Min8. This approach was applied to mitochondria harboring FLAG-tagged Min8 and, hence, combined with immunoprecipitation of Min8. The analysis via Western blotting revealed distinct shifts of Min8. These shifts were induced by oxidative cross-linking with other proteins that were identified by LC-MS/MS (see subsection 3.1.10, Figure 18a). It has to be noted that in all analyzed bands Min8 was identified. Strikingly, within the 30 most abundant and shifted proteins, i.e. proteins that did not show the expected migration in SDS-PAGE but an $8 \mathrm{kDa}$ shift, Cox2 as subunit of CIV as well as Rcf1 and Rcf2 could 
4.4 Protein-protein cross-links in mitochondria derived from yeast grown on either glycerol- or glucose-containing medium

be identified (Rcf proteins will be discussed below). Other proteins that could be identified by this approach were e.g. Сyc1, Сyc3 and Tim11. All mentioned proteins belong to the OXPHOS system. Not only the shifted proteins were considered, but also those that coimmunoprecipitated without showing an induced shift. To increase the reliability of these data, only those proteins were considered as co-immunoprecipitated that were identified by at least seven peptides in the respective fraction. Amongst these proteins, subunits of CIII, CIV and CV and also Pet9 were identified (see subsection 3.1.10, Figure 18b). By oxidative cross-linking an interaction between Min8 and Cox2 could be identified. Within the structure that was proposed by the docking experiment (see subsection 3.1.10, Figure 17) the closest cysteine residue to Min8 is indeed $\mathrm{C} 107$ of Cox2. However, the distance between the sulfur atom of Cox2's C107 and the sulfur atom of Min8's C36 is $20.7 \AA$ in the model. In the presented model, Min8 would have to be rearranged towards Cox2 to explain a disulfide bridge induced by $\mathrm{Cu}(\mathrm{II})$ ions with a bond length of approximately $2 \AA$. To support the results from the oxidative cross-linking approach, Cys-Cys-specific crosslinkers such as BMOE or BMH that form non-cleavable covalent bonds might be applied. With that, similar to BS3-induced cross-links, a direct connection between peptides could be identified and a larger distance could be spanned. The proposed model of Min8 and its association with CIV based on the cross-links indicate that results gained by XL-MS can be successfully used for docking experiments to obtain compelling models of protein complexes even with a limited number of distance constraints.

As recently as in 2016, Levchenko et al. ${ }^{124}$ and Strecker et al. ${ }^{125}$ identified a new supercomplex-associated protein, Cox26, which is part of the yeast $\mathrm{CIII}_{2} \mathrm{CIV}_{2}$ supercomplex structure ${ }^{91}$. Interestingly, the length of Min8 (72 amino acids) and the modeled helical structure are very similar to the ones of Cox26 (66 amino acids). This raises the hypothesis that Min8 is not transiently associated with but is a novel integral subunit of CIV.

Alternatively, Min8 might aid solely the assembly of CIV. In Linden et al. ${ }^{247}$, the authors present evidence for Min8 being an assembly factor, in particular for the assembly of Cox12 into CIV. To evaluate Min8's biological function, a min8s strain was biochemically investigated in the laboratory of Prof. Dr. Peter Rehling, Cellular Biochemistry, University Medical Center Göttingen. A detailed description of the results and an extensive discussion to these experiments can be found in the PhD thesis performed by Bettina Homberg with the preliminary title 'Regulation of mitochondrial supercomplex formation'. Bettina Homberg, Cellular Biochemistry, University Medical Center Göttingen is part of the GGNB program 'Molecular Medicine'. Briefly, cells lacking Min8 did not show any growth or respiration defect. However, it could be demonstrated that Min8 positively affects the assembly of Cox12 and negatively affects the assembly of 
Cox13, both peripheral subunits of CIV and added to mature CIV in a late stage ${ }^{130}$. In general, the assembly of CIV is a complex process and not yet fully understood. In yeast, CIV consists of twelve subunits. Three of them, the catalytic core subunits Cox1-3, are encoded by the mtDNA. The other subunits are encoded by nuclear DNA and imported into the mitochondria. These subunits increase stability and activity of the complex as well as reduce the production of reactive oxygen species $(\mathrm{ROS})^{115,132,343}$. Additionally, more than 30 translation regulators and assembly factors are necessary ${ }^{126,344,345}$. Some of them were identified in the past, such as Coa $1^{135}$, Coa2 ${ }^{136}$, Coa3 ${ }^{133,346}, \operatorname{Cox} 14^{347,348}$, Shy $1^{134}$, Mss $51^{349}$ and Pet309350 in regard to Cox1 $1^{122}$, resulting in intermediate structures. Intermediates are then also formed between CIV subunits on the path to fully assembled CIV, such as Cox1-Cox5-Cox6 or Cox7-Cox8-Cox9, which are, in turn, also supported by assembly factors ${ }^{127-129,351}$. More recently, also factors for the assembly of Cox13 and supercomplex formation between CIII and CIV have been identified ${ }^{115,119}$, namely respiratory supercomplex factors Rcf1, Rcf2 and Rcf3. Especially for Rcf1 it could be demonstrated that the interaction to CIV is rather transient, altering its lipid composition that, in turn, increases the complex' activity ${ }^{352}$. Remarkably, cross-links between Rcf2 and both Cox12 and Cox13 as well as between Rcf3 and Cox12 could also be identified in this thesis (see subsection 3.1.5, Figure 10 and subsection 4.4.6). Therefore, it is also conceivable that Min8 is a new member of the Rcf proteins that dynamically interacts with CIV. The assembly of Cox12 into CIV promoted by Min8 seems to occur in a yet undefined new intermediate that was identified in the Cox12 import assay (described in subsection 3.1.10 and in more detail in Bettina Homberg's PhD thesis). Radiolabeled Cox12 could be identified in a complex that migrated faster than mature CIV after full solubilization with DDM. This behavior was detected in all tested conditions, wild type, min8s strain and $\operatorname{cox} 4 \Delta$ strain. Especially in the cox $4 \Delta$ strain, no mature CIV can be assembled ${ }^{353}$, hence, identified radiolabeled Cox12 is not assembled within mature CIV. The detected intermediate could have different origins: first, it could be another yet undescribed intermediate of CIV that exists without Cox4. This is supported by the fact that this intermediate is enriched in the $\operatorname{cox} 4 \Delta$ strain. Second, it could be an intermediate during Cox12 import. Unlike other CIV subunits, Cox12 is not a transmembrane protein but is localized in the IMS ${ }^{143}$. Its import is also not inhibited by interfering the membrane potential but by inhibiting the MIA pathway. MIA, mitochondrial intermembrane space import and assembly system, consists of the receptor Mia40 and the sulfhydryl oxidase Erv1 and is responsible for import and folding of IMS proteins ${ }^{58,354}$. Therefore, it is conceivable that MIA aids the import of Cox12, and the so far uncharacterized intermediate is a result of Cox12 import. Previous studies could demonstrate that MIA substrates show a $\mathrm{Cx}_{3} \mathrm{C}$ or $\mathrm{Cx}_{9} \mathrm{C}$ motif ${ }^{58,355,356}$, as is the case e.g. for the IMS proteins Tim9 and Tim10, and also for 
Cox12. However, if the intermediate represented an import intermediate, expected proteins within this intermediate were Mia40, Erv1, Cox12 as substrate, Min8 as assembly promoter for Cox12 and probably cytochrome $c$ that reoxidizes Erv $1^{58}$. This would end up in a total molecular mass of approximately $95 \mathrm{kDa}$ that does not correspond to the observed intermediate (molecular mass approximately $150 \mathrm{kDa}$ ). Ultimately, Min8 promotes Cox12's assembly to this newly identified intermediate. More experiments are needed to fully clarify the origin of this intermediate. An LC-MS/MS analysis of separated proteins after a BN-PAGE/SDS-PAGE approach could shed light on the protein composition of this intermediate. However, the challenge here is to enrich this intermediate in a sufficient amount. (At this point I would like to emphasize that especially the second hypothesis was not solely my own idea but developed in a discussion with Bettina Homberg.) While writing this thesis, another structure of the yeast $\mathrm{CIII}_{2} \mathrm{CIV}_{(2)}$ supercomplex was solved by cryo-EM ${ }^{120}$. In this structure obtained from yeast cells expressing the hypoxic isoform Cox5b, Rcf2 could be identified at the periphery close to the position of Cox13. The position of Rcf2 does not interfere with the modeled position of Min8 attached to CIV presented in this thesis. This brings up the question, whether Rcf2 helps in the assembly of CIV or the supercomplex or whether it is a stoichiometric subunit of CIV. Rcf1, in contrast, has a regulatory function on CIV. Rcf1 leaves the mature complex ${ }^{118}$ and is not present in cryo-EM 3D structures. Also, no interaction between Rcf1 and CIV has been detected by cross-linking yet. Whether Min8 is another stoichiometric subunit of CIV similar to Rcf2 or a regulatory protein such as Rcf1 still needs further evaluation.

\subsubsection{The pyruvate dehydrogenase complex}

The focus of this subsection lies on the pyruvate dehydrogenase complex (PDH). In mitochondria from yeast grown on glucose-containing medium the number of cross-links within the PDH were higher than in mitochondria from yeast grown on glycerol-containing medium (see subsection 3.1.7, Figure 14). Saccharomyces cerevisiae can generate energy in form of ATP via two essential metabolic pathways: fermentation and oxidative phosphorylation. In the following, both pathways are discussed. Fermentation is triggered by high sugar concentrations such as glucose. Glucose represses transcription of genes that are involved e.g. in oxidative phosphorylation and in the TCA cycle $e^{75,76,321}$ and is mainly metabolized via fermentation ${ }^{357,358}$. Here, pyruvate as product of the glycolysis is preferably decarboxylated by the pyruvate decarboxylase (Pdc) producing acetaldehyde that is finally oxidized by the alcohol dehydrogenase to ethanol ${ }^{358}$ resulting in 2 equivalents of ATP $^{359}$. Other reactions are via the cytosolic aldehyde dehydrogenase that oxidizes acetaldehyde to acetate. Acetate is then further processed by acetyl-CoA synthetase to 
acetyl-CoA, the precursor of the TCA cycle, by-passing the pyruvate dehydrogenase complex $(\mathrm{PDH})$ in mitochondria ${ }^{74,358}$. Pyruvate can also immediately be transported into mitochondria. Here, the PDH uses pyruvate as substrate to form acetyl-Co $A^{74,358}$ which is then channeled into the TCA cycle and finally ends up in the oxidative phosphorylation system resulting in 38 equivalents of ATP359.

From a metabolic point of view, the PDH seems to be more important for yeast cells growing on non-fermentable carbon sources like glycerol. However, cross-linking data obtained in this thesis indicate that a fully assembled PDH (cross-links between all three enzymes E1, E2, E3 and the E3-binding protein Pdx1) is more abundant under glucose condition (see subsection 3.1.7, Figure 14). Competitors for pyruvate metabolism in yeast are the pyruvate decarboxylase in the cytosol and the pyruvate dehydrogenase complex in the mitochondrial matrix. Although showing a higher $\mathrm{K}_{\mathrm{m}}$ value for binding pyruvate than $\mathrm{PDH}^{360-362}$, $\mathrm{Pdc}$ is the prime enzyme for pyruvate consumption in fermentation ${ }^{358}$. $P d c^{-}$cells showed severe growth defects on fermentable carbon sources $^{363}$ indicating that the PDH cannot produce enough acetyl-CoA alone ${ }^{74}$. In turn, yeast cells with mutations of genes encoding PDH subunits resulting in the loss of PDH activity were all viable on both fermentable and non-fermentable carbon sources ${ }^{280,364-366}$. However, a pda $1^{0}$ mutant that lacks the $\alpha$-subunit of the E1 pyruvate dehydrogenase enzyme showed a slightly decreased growth rate compared with the wild type ${ }^{366}$. This defect could be rescued by the exogenous addition of the amino acid leucine. Furthermore, the mutant showed instability of the mitochondrial genome, similar to a $r h o^{\circ}$ strain. This indicates Pda1's role in branched amino-acid synthesis and genome maintenance in mitochondria. Remarkably, Ilv5 has a comparable dual role in branched amino acid biosynthesis and genome maintenance ${ }^{46}$ (see subsection 4.5.1). In summary, the PDH is not solely involved in pyruvate metabolism.

Glycerol catabolism is initiated by the glycerol kinase Gut1 and the FAD-dependent glycerol 3-phosphate dehydrogenase Gut2 ${ }^{367,368}$. Both proteins are repressed by glucose but highly abundant under glycerol condition 328,369 . The reaction cascade performed by these two enzymes transforms glycerol to dihydroxyacetone phosphate which can then be channeled to the glycolysis pathway ${ }^{79,367,368}$. Since respiratory enzymes are not repressed under glycerol condition, energy is mainly produced by oxidative phosphorylation. AcetylCoA as substrate for the TCA cycle is mainly produced by the PDH that uses pyruvate provided by glycolysis ${ }^{370}$. Another fact that has to be mentioned is that FAD-dependent Gut2 delivers two electrons from glycerol to the ETC, by-passing the TCA cycle ${ }^{78}$. Similar to the cytosolic aldehyde dehydrogenase that oxidizes acetaldehyde to acetate via the PDH-by-pass pathway, also a mitochondrial aldehyde dehydrogenase exists. Via this dehydrogenase, Ald4, the PDH can also be by-passed ${ }^{80}$. The Pdc that is also functional 
4.4 Protein-protein cross-links in mitochondria derived from yeast grown on either glycerol- or glucose-containing medium

under non-fermentable conditions generates acetaldehyde from pyruvate. Acetaldehyde is then further oxidized to acetate by mitochondrial Ald4, reducing $\mathrm{NAD}^{+}$in the matrix. Acetate can then be further processed to acetyl-CoA by the acetyl-CoA synthetase in the cytosol. Yeast cells with a co-disruption of the ALD4 and PDA1 genes are not viable on non-fermentable carbon sources ${ }^{371}$.

The comparison of both the fermentable and the non-fermentable metabolic pathways indicate that the PDH is important for both. Accordingly, copy numbers of E1 and E2 of the PDH are equal under both conditions on cell level ${ }^{72}$. However, E3 is more abundant under glycerol condition but is also part of other a-oxoacid dehydrogenases, thus, is also a subunit of the $\alpha$-ketoglutarate dehydrogenase in the TCA cycle ${ }^{372,373}$. It is therefore difficult to estimate how many copies of $\mathrm{E} 3$ are involved in which complex. The E3-binding protein Pdx1, in turn, has a 1.4-fold higher copy number under glucose condition $^{72}$ (see subsection 3.1.7, Figure 13). Pdx1 helps in the assembly of E2 and E3, finally forming a functional $\mathrm{PDH}^{280}$. The higher number of cross-links found between $\mathrm{Pdx} 1$ and E2 as well as E3 under glucose condition is consistent with the function of Pdx1 in the assembly of the PDH. However, one would expect the opposite picture, that is, a fully assembled PDH as reflected by the numbers of cross-links of Pdx1 to E2 and E3 should be higher under glycerol condition. A possible explanation for these controversial results could be that the PDH is metabolically equally important for both growth conditions, but in regard of its involvement also in amino acid biosynthesis and genome maintenance in mitochondria, the PDH might play a key role for cells growing on fermentable carbon sources. These cells grow faster than under non-fermentable conditions ${ }^{374-376}$ and therefore might have a higher demand for e.g. amino acids. Accordingly, higher abundances of proteins involved in amino acid biosynthesis under glucose condition were identified in the proteomic analysis in this thesis (see section 3.4) and also the numbers of cross-links of proteins involved in amino acid biosynthesis were higher under glucose condition (see subsections 3.1.3, 3.1.6, 4.4.3, and 4.5.1). It can be hypothesized that the differential expression of the E3-binding protein $\mathrm{Pdx} 1$ might represent a regulation step for the assembly of the PDH. E1 and E2 are equally abundant suggesting that a fully functional $\mathrm{PDH}$ is present under both conditions. Conversely, cross-links between Pdx1 and E2 as well as E3 indicate a pronounced interaction under glucose rather than under glycerol condition. Such discrepancy could be explained by an additional function of the PDH in amino acid biosynthesis and genome maintenance that are more pronounced under glucose condition.

The equal abundances of PDH subunits under glycerol and glucose conditions and the higher number of cross-links within the PDH under glucose condition raise the question how yeast cells grown on glycerol-containing medium produce enough energy in terms of 
ATP. One of the most abundant proteins under glycerol condition in the proteomic analysis (see section 3.4) and also in the quantitative approach (see subsection 3.1.6) was Ald4. In a direct comparison, the copy number of Ald4 is 17-fold higher under glycerol condition compared to glucose condition in cells ${ }^{72}$. This suggests that yeast cells grown on glycerolcontaining medium also use the $\mathrm{PDH}$-by-pass pathway for acetyl-CoA generation in mitochondria and not only the PDH alone - similar to the yeast cells grown on glucosecontaining medium that use the PDH-by-pass pathway via the Pdc.

\subsubsection{Interactions of uncharacterized proteins}

Cross-links of proteins that are so far uncharacterized or have been partially characterized in recent years as listed in the Saccharomyces Genome Database (SGD) ${ }^{29}$ have also been identified in this thesis (see subsection 3.1.8 and Supplementary Figure 5). Cross-linking data presented in this thesis provide evidence for interaction partners of those uncharacterized proteins and help to put them into their biological context. Some of the described cross-links have also been identified by Makepeace et al. ${ }^{102}$ Min8 and its interactions to Cox12 and Pet9 (see subsection 4.4.4) provide a compelling example how cross-links can be utilized for protein characterization. Also, the cross-links of recently identified Rcf2 and its N-terminal homolog Rcf3 were briefly discussed in the same subsection. While the N-terminus of Rcf3 cross-linked to the N-terminus of Cox12, Rcf2 interacted C-terminally with both Cox12 as well as Cox13. All identified interactions took place in the IMS. Cross-linking data are in accordance with the proposed inner membranous localization and orientation of both Rcf proteins proposed by Römpler et al. ${ }^{119}$. The authors demonstrated that Rcf2 is processed after import into an unstable Nterminal and a stable C-terminal part, both protruding into the IMS. The cross-links indeed indicate an interaction of the stable C-terminal part of Rcf2 to Cox12. In summary, Rcf2's and Rcf3's association with CIV could be confirmed by the cross-links illustrated in this thesis.

Cross-links were also identified between Nat2 and Mdh1, the malate dehydrogenase within the TCA cycle. Nat2 is a protein of so far unknown function but listed as a putative $\mathrm{N}^{\alpha}$-acetyltransferase ${ }^{377}$ in the SGD. According to Vögtle et al. ${ }^{142}$, Nat2 localizes to the mitochondrial IM. $\mathrm{N}^{\alpha}$-acetyltransferases post-translationally modify $68 \%$ of all yeast proteins at their $\mathrm{N}$-terminus by adding an acetyl moiety ${ }^{378}$. Although serine, alanine and methionine residues are preferred substrates, there is no consensus sequence in general. The cross-links suggest that both proteins are in close proximity to each other and thereby the possibility that Nat2 acetylates Mdh1. However, after cleavage 
4.4 Protein-protein cross-links in mitochondria derived from yeast grown on either glycerol- or glucose-containing medium

of the mitochondrial presequence, Mdh1's N-terminus starts with a tyrosine residue and an $\mathrm{N}$-terminal acetylation of Mdh1 has not yet been reported.

Ycp4, another protein of unknown function, cross-linked to Pst2 and Hsp60. Hsp60 is a chaperonin that might aid folding of $Y_{c p 4}$; Pst2 is a flavodoxin-like protein. Interestingly, Ycp4 is listed as a putative flavodoxin-like protein in the SGD. Ycp4 and Pst2 were both localized to the $\mathrm{IM}^{142}$. Flavoproteins are proteins containing FAD or FMN prosthetic groups for electron transport, most of them localized in mitochondria ${ }^{379}$. The function of Ycp4 and Pst2 still needs to be elucidated. Remarkably, cross-links between Ycp4 and Pst2 were also identified by Makepeace et al. ${ }^{102}$.

While the aforementioned cross-links were all identified in the glycerol data set, the following were identified in the glucose data set. Aim17, a protein of unknown function, cross-linked to $\mathrm{Cpr} 3$, the mitochondrial peptidyl-prolyl cis-trans isomerase $\mathrm{C}$, both localized to the matrix ${ }^{142}$. Cpr3, also known as cyclophilin, accelerates protein folding ${ }^{380}$. Aim17 could be a potential substrate.

Another cross-link was detected between Coq21 and Coq5. Morgenstern et al. ${ }^{72}$ could already identify Coq21 as matrix protein. By performing immunoprecipitation of Coq21, the authors could also prove its association with the coenzyme $\mathrm{Q}$ biosynthesis cluster. Coenzyme $Q$ is a lipid with redox activity participating e.g. in the $\mathrm{ETC}^{381}$. The identified cross-link supports Coq21's participation in that cluster.

Dpi8 is another protein that was localized to the matrix by Morgenstern et al. ${ }^{72}$. It was identified as interaction partner of $\mathrm{Kgd1}$, a subunit of the a-ketoglutarate dehydrogenase of the TCA cycle, through cross-links in this thesis and in the study of Makepeace et al. ${ }^{102} \mathrm{Dpi} 8$ is a comparably small protein with a molecular weight of $7.7 \mathrm{kDa}$. A putative function of this protein could be in aiding the assembly of $\alpha$-ketoglutarate dehydrogenase complex.

Fmp16 is also a protein of unknown function and cross-linked to Aco1, the aconitase of the TCA cycle. Cross-links between these proteins were also identified by Makepeace et al. ${ }^{102}$ Aconitase transforms citrate to isocitrate in one of the first steps within the TCA cycle. Besides that, Aco1 is also involved in genome maintenance ${ }^{45}$. Interestingly, in the rho data set, Aco1 cross-linked also to Mmf1 (see subsections 3.2.2 and 4.5.1), another protein involved in genome maintenance ${ }^{382}$. Fmp16 could therefore either be involved in TCA regulation mechanisms or also in genome maintenance.

The ORF YDR061W encodes an uncharacterized protein. In this thesis, two unique cross-links were identified to llv5, the mitochondrial keto-acyl reductoisomerase involved in the amino acid biosynthesis pathway of isoleucine and valine and in genome maintenance ${ }^{46}$. The protein encoded by YDR061W has a molecular weight of $61 \mathrm{kDa}$, similarity to $A B C$ transporters, an ATP binding site ${ }^{383}$ and is localized to the $\mathrm{IM}^{142}$. 
By applying the protein size exclusion chromatography approach to improve the analysis depth after cross-linking of mitochondria, additional cross-links of other proteins of unknown function could be identified (see section 3.3). Among these are cross-links of Fmp10 to Mrx3. Mrx3 is associated with the mitochondrial ribosome and might be involved in cristae junction formation ${ }^{384}$. Other cross-links were identified between Fmp40 to Ald4, the aldehyde dehydrogenase and between Fmp41 and Ssc1, a heat shock protein involved in protein folding ${ }^{385}$. Fmp41 could be a potential substrate of Ssc1.

A multitude of cross-links were identified between Om45 and Nde1 under both growth conditions revealing a tight interaction between these proteins (22 and 11 unique residue-to-residue cross-links in the glycerol and the glucose condition, respectively). Although Om45 is a highly abundant protein of the outer mitochondrial membrane ${ }^{278}$, it has been barely characterized so far. Deletion of Om45 has no significant effect on mitochondrial function or cellular growth regardless of the carbon source ${ }^{278}$. While the localization of $\mathrm{Nde1}$, the external $\mathrm{NADH}$ :ubiquinone oxidoreductase, to the $\mathrm{IM}^{142}$ and its orientation to the IMS ${ }^{386}$ is clearly defined, the orientation of Om45 is still under debate ${ }^{387,388}$. Studies in the past reported different results supporting either Om45's protrusion into the cytosol ${ }^{278,387,389,390}$ or into the $\mathrm{IMS}^{279,391,392}$. Results in particular obtained from the laboratories of Professor Nikolaus Pfanner and Professor Toshiya Endo could demonstrate a localization of Om45 to the IMS by applying high concentrations of proteinase $\mathrm{K}$ to mitochondria without a signal loss of Om45 in Western blot analyses ${ }^{391,392}$. The cross-links identified in this thesis corroborate Om45's IMS orientation and additionally indicate a strong interaction with Nde1 in the IMS. Cross-links between Om45 and Nde1 were also identified by Makepeace et al. ${ }^{102}$ Biochemical studies performed by Lauffer et al. ${ }^{279}$ could show an interaction between Om45 and Por1 proposing that Om45 might help in Por1's regulation. This interaction was confirmed in this thesis by cross-links between Om45 and Por1 in both conditions (see subsection 3.1.4, Figure 9). Since Om45 crosslinked to both Por1 and Nde1, Om45 could function as a guide for Nde1 to get into close proximity to Por1. This might accelerate the oxidation of cytosolic NADH to $\mathrm{NAD}^{+}$by $\mathrm{Nde} 1^{393}$ and the shuttling of these redox equivalents by Por $1^{394}$ back to the cytosol. Nde1 can then feed the electrons to the respiratory system, e.g. to cytochrome $c$ (Cyc1). One cross-link between Cyc1 and Nde1 was identified in the glucose data set (Cyc1 was not part of the databases for the glycerol data set and technically could not have been identified although an interaction between Cyc1 and Nde1 might also have existed in the glycerol condition). This would also explain the higher intensity of Om45-Nde1 interprotein cross-links in the glycerol condition as identified in the quantitative approach (see subsection 4.6.2). However, more biochemical experiments are needed to give a wellgrounded prediction on Om45's function and whether this is ultimately related to Nde1. 
4.4 Protein-protein cross-links in mitochondria derived from yeast grown on either glycerol- or glucose-containing medium

\subsubsection{Protein size exclusion chromatography slightly improved analysis depth}

Protein size exclusion chromatography (protein SEC) was applied to cross-linked mitochondria derived from yeast grown on glycerol-containing medium to increase the analysis depth. Indeed, the number of CSMs drastically increased (see section 3.3, Table 9). However, the number of proteins involved in cross-linking reactions increased only slightly and the number of unique residue-to-residue cross-links even decreased slightly. The resolution of a protein size exclusion chromatography is comparably low. Although the cross-linked sample was prefractionated on protein level before further analysis, the resolution was probably not high enough to separate abundant protein complexes (such as ETC complexes) from lower abundant complexes (such as TOM/TIM complexes). Moreover, proteins were cross-linked prior to protein SEC which presumably makes it even more difficult to separate cross-linked proteins or complexes from each other. For all protein SEC fractions, a database with the 400 most abundant proteins were used for the cross-linked peptides searches (see section 4.3). Although databases were protein SEC fraction-specific and in total more proteins were covered compared to the peptide SEC approach (also because more fractions resulted in increased measurement time), the coverage of the mitochondrial proteome was not as complete as desired. This is illustrated by an average protein overlap of $69 \%$ between databases of consecutive fractions ranging from $54 \%$ in the later fractions with presumably less cross-linked proteins and $80 \%$ in the earlier fractions containing cross-linked proteins. To improve the analysis depth, a more dedicated fractionation on protein level should be applied such as the separation in membrane-bound and soluble protein fractions after cross-linking as performed by Makepeace et al. ${ }^{102}$

Nonetheless, some protein-protein cross-links were detected only in the protein SEC approach. For example, Cox13 cross-linked to Mia40, a subunit of the MIA import pathway. While this was already discussed for Cox12 (see subsection 4.4.4), a transport of Cox13 into mitochondria via MIA could biochemically not be proven. The import of Cox13 is dependent on the membrane potential, thus, indicating a transport via the TIM machinery ${ }^{68}$ and not via the MIA system. During the cross-inking reaction, Cox13 and Mia40 must have been in close proximity to each other but its biological relevance is unclear so far.

A cross-link between Mic10, component of the mitochondrial contact-site and cristae-organizing system (MICOS), and Tim11, a subunit of the $\mathrm{F}_{1} \mathrm{~F}_{0}$ ATP synthase (CV), was detected. Both MICOS 16,17,395,396 and $\mathrm{CV}^{20,397,398}$ due to its V-shaped ${ }^{399}$ dimer formation ${ }^{18,400,401}$ form the shape of the IM, especially the cristae. Unfortunately, no further 
cross-links of MICOS proteins were detected. MICOS consists of six proteins in yeast ${ }^{402}$ and is supposed to interact with channels located in the OM. Yeast mutants that are MICOS deficient show no cristae formation. The interplay between MICOS and CV and therefore its impact on crista junction regulation have recently been described ${ }^{19,290}$. Remarkably, both studies could identify the same interaction between both complexes (Mic10 and Tim11) by chemical cross-linking combined with co-immunoprecipitation experiments. It is suggested that Mic10 helps in oligomerization of CV that, in turn, is mediated by subunits Tim11 and Atp20.

The protein SEC approach also revealed that the N-terminus of Por1 cross-linked to the N-terminus of Min6. Min6 is supposed to be a peroxisomal protein with unknown function ${ }^{289}$. Recently, it could be identified as a protein of the mitochondrial OM by MSbased proteomics ${ }^{72}$. Based on the cross-link in this thesis, it is difficult to support one of these hypotheses. While the N-terminal segment of Por1 is supposed to be involved in a conformational process defining the open and the closed state ${ }^{403}$, it is still under discussion if the N-terminus is localized in the IMS or in the cytosol ${ }^{308,404-406}$. Since the orientation of Min6 is also undefined so far, both the mitochondrial OM and the peroxisomal membrane cross-talking with mitochondria ${ }^{407}$ are putative localizations.

In addition to the abovementioned putative mitochondria-peroxisomes cross-link, a cross-link between the mitochondrial OM protein Om45 and the endoplasmic reticulum (ER) protein Sss1, a subunit of the Sec61 translocation complex ${ }^{288}$, was detected. The Sec61 translocation complex is involved in ribosome binding and subsequent cotranslational translocation of proteins to the $\mathrm{ER}^{408,409}$. Since the C-terminal segment of Om45 was cross-linked to Sss1, this would interfere with the hypothesis that Om45 is located in the IMS and tethered to the OM with its $\mathrm{N}$-terminal segment (see subsection 4.4.6). Interactions between the ER and mitochondria already have been reported in the past. These Interactions are mainly mediated by the ER-mitochondria encounter structure (ERMES) that might contribute to lipid transfer ${ }^{410}$.

\subsubsection{A technical comparison with other studies dealing with XL-MS of mitochondria}

During the course of this thesis, four additional studies that applied XL-MS to mitochondria have been published. Table 11 summarizes information about these studies in comparison to this thesis, clustered in origin of the cross-linked mitochondria, the used cross-linker in combination with analysis software suites, number of cross-links and FDR calculation. Mitochondria isolated from mouse ${ }^{99,100}$, human ${ }^{101}$ and yeast ${ }^{102}$ (including the published results ${ }^{247}$ of this thesis) have been investigated. In these five studies considered, five 
4.4 Protein-protein cross-links in mitochondria derived from yeast grown on either glycerol- or glucose-containing medium

different cross-linkers were used, three of them MS-cleavable (see subsection 4.8.2). Also, five different software suites were used for the identification of cross-linked peptides with five different strategies to calculate the false discovery rate (FDR). This already demonstrates the plethora of possible combinations in cross-linking experiments. It is therefore not surprising that different numbers of identified protein-protein cross-links are reported.

Schweppe and colleagues from the laboratory of Professor James Bruce were the first ones who cross-linked murine mitochondria ${ }^{99}$. The authors used their in-house developed protein interaction reporter (PIR) technology ${ }^{223}$ with the enrichable and MScleavable cross-linker BDP-NHS ${ }^{206}$. With this approach, the authors were able to identify 459 unique protein-protein cross-links, $52 \%$ of them interprotein cross-links.

An MS-cleavable but not enrichable cross-linker, DSSO ${ }^{221}$, was used by Liu et al. ${ }^{100}$ in combination with their in-house developed software suite XlinkX ${ }^{411}$, also to analyze murine mitochondria. The authors almost doubled the protein-protein cross-links in comparison to Schweppe et al. to 885 with an outstanding $69 \%$ of them being interprotein cross-links. Both studies used an advanced tribrid mass spectrometer to select specific fragment ions after cleavage of the cross-linker. Cross-linked peptides were then identified based on MS3 spectra, but different FDR cut-offs were applied.

Human mitochondria were cross-linked with the non-cleavable cross-linker DSS by Ryl et al. ${ }^{101}$ The cross-linker used in this thesis, BS3, is a sulfonated derivative of DSS. DSS and BS3 provide the same reaction chemistry as well as same distance constraints. The number of identified protein-protein cross-links, 863 , is comparable to the results provided by Liu et al. in murine mitochondria, but, surprisingly, only $10 \%$ of them represented interprotein cross-links (see section 4.7). For identification of cross-linked peptides, the authors used their in-house developed software $\mathrm{Xi}^{412}$. Remarkably, Ryl et al. could identify 792 proteins involved in cross-linking reactions, almost doubled in comparison to this thesis and the studies by Schweppe et al. and Liu et al. This could be due to an extensive fractionation in combination with sequential digestion ${ }^{327}$. Ryl et al. combined peptide SEC with SCX fractionation and used trypsin alone and in combination with another protease (AspN, GluC, or chymotrypsin). This increased the number of peptides enabling the identification of proteins that would not have been identified after digestion with trypsin alone. However, the number of identified interprotein cross-links is comparably low. This, in turn, could be due to the applied FDR strategy. In contrast to Schweppe et al., Liu et al. and the FDR strategy used in this thesis, Ryl et al. calculated the FDR on residue level, and, in addition, separately for intraprotein and interprotein cross-links. While this approach is more accurate - FDR should be calculated on the level of interest, in fact on PPI level ${ }^{413}$ - it reduces the number of interprotein cross-links ${ }^{101}$ (a 
biological comparison between the results of cross-linked human mitoplasts within this thesis and the work performed by Ryl et al. will be discussed in section 4.7).

Makepeace et al. ${ }^{102}$ analyzed, similar to the work presented in this thesis, mitochondria isolated from yeast. They presented the so far highest number of involved proteins (811) and protein-protein cross-links (1122) with $30 \%$ interprotein cross-links. The authors used their in-house synthesized MS-cleavable, enrichable, and isotopically labeled cross-linker CBDPS ${ }^{216}$ in combination with their software suite Qualis-CL and applied a $2 \%$ FDR cut-off on peptide-level. Makepeace et al. performed extensive fractionation already on protein-level by separating the soluble fraction from two different membrane fractions. This might be a reason for the high numbers of identifications. In their study, the authors rather focused on the cross-linking workflow and the identification of cross-linked peptides than on the biological interpretation of the data. $76 \%$ of the proteinprotein cross-links detected in this thesis are covered by the work of Makepeace et al. Specific results are discussed in section 4.4 .

In conclusion, this comparison shows how diverse the cross-linking field is in regard to used cross-linkers, software suites, and FDR calculation. In comparison to all other studies, the number of identifications presented in this thesis are the lowest. This is mainly due to different fractionation methods, cross-linked peptides enrichments and FDR calculation strategies. However, with the lowest FDR of $1 \%$ and the applied filtering steps (see subsection 4.4.1) the results presented in this thesis are the most conservative ones. Despite all the differences between the studies, the overlap of $76 \%$ of protein-protein cross-links compared to the study performed by Makepeace et al. demonstrates that cross-linking results from two different laboratories can also be reproducible. 
Table 11: Comparison between so far published studies dealing with XL-MS of mitochondria. Prot, protein; FDR, false discovery rate.

\begin{tabular}{|c|c|c|c|c|c|c|c|}
\hline & & glycerol $^{247}$ & glucose $e^{247}$ & Schweppe et al. ${ }^{99}$ & Liu et al. ${ }^{100}$ & Ryl et al. ${ }^{101}$ & $\begin{array}{r}\text { Makepeace } \\
\text { et al. } .^{102}\end{array}$ \\
\hline origin & organism & yeast & yeast & mouse & mouse & human & yeast \\
\hline \multirow{6}{*}{ 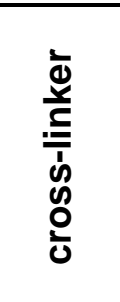 } & cross-linker & BS3 & BS3 & BDP-NHP & DSSO & DSS & CBDPS \\
\hline & cleavable & no & no & yes & yes & no & yes \\
\hline & enrichable & no & no & yes & no & no & yes \\
\hline & labeled & no & no & no & no & no & yes \\
\hline & MS level & MS2 & MS2 & MS3 & MS3 & MS2 & MS2 \\
\hline & software & pLink 1 & pLink 1 & Comet & XlinkX2.0 & $X i$ & Qualis-CL \\
\hline \multirow{4}{*}{ 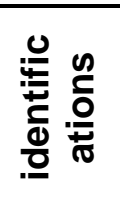 } & involved proteins & 261 & 260 & 327 & 359 & 792 & 811 \\
\hline & prot-prot cross-links & 396 & 386 & 459 & 885 & 863 & 1122 \\
\hline & intraprot cross-links & $229(58 \%)$ & $231(60 \%)$ & $222(48 \%)$ & $276(31 \%)$ & $773(90 \%)$ & $784(70 \%)$ \\
\hline & interprot cross-links & $167(42 \%)$ & $155(40 \%)$ & $237(52 \%)$ & $609(69 \%)$ & $90(10 \%)$ & $338(30 \%)$ \\
\hline \multirow{4}{*}{ 孚 } & FDR & $1 \%$ & $1 \%$ & $<5 \%$ & $2 \%$ & $5 \%$ & $2 \%$ \\
\hline & FDR level & spectrum & spectrum & spectrum & spectrum & residue & peptide \\
\hline & separate FDR & no & no & no & no & yes & no \\
\hline & user defined filtering & yes & yes & no & no & no & no \\
\hline
\end{tabular}




\subsection{Protein-protein cross-links in mitochondria derived from a $r h o^{\circ}$ yeast strain}

The initial idea behind the use of mitochondria from a $r o^{\circ}$ yeast strain for cross-linking was to identify interactions between mitochondrial proteins and protein complexes that have not been detected in mitochondria isolated from yeast grown on glycerol- or glucosecontaining medium. It was assumed to cross-link also lower abundant complexes such as the TOM/TIM complex or MICOS. The $r o^{\circ}$ strain is unable to grow on non-fermentable carbon sources such as glycerol because of the strain's inability for respiration. Additionally, due to the lack of rRNAs the mitoribosomal proteins cannot assemble to a functional ribosome. Accordingly, less cross-links were identified within the OXPHOS system in comparison to the yeast wild type strains grown under glycerol- or glucoseconditions (see subsection 3.1.5, Figure 10). Within CIII and CIV only cross-links between subunits Cor1 and Qcr2 as well as Cox5b and Cox6 were identified, underlining their preassembly before their integration into mature complexes ${ }^{122,127,128,414}$. Cross-links within Cll was identified between subunits Sdh1, Sdh2 and Sdh3. The high coverage of Cllsubunits can be explained by Cll's dual role, that is, to participate not only in the ETC but also in a functional TCA cycle $e^{415}$. Also, less cross-links within subunits of the mitoribosome were identified in the $r \mathrm{ro}^{\circ}$ strain. In comparison to the glycerol and glucose data sets, in which 33 and 31 mitoribosomal proteins were involved in cross-linking reactions, respectively, only two of these proteins were identified in the $r h 0^{\circ}$ data set (data not shown). These data are in line with the biological context, that is, the lack of mtDNA in $r h o^{\circ}$ yeast cells and the effect on the respiratory system and the mitochondrial ribosome.

\subsubsection{Energy metabolism and amino acid biosynthesis pathways - or a dual role for mitochondrial proteins?}

At a first glance, in the $r h o^{\circ}$ data set, cross-links within and between proteins that are involved in the TCA cycle and in the pathway for the biosynthesis of the amino acids valine and isoleucine were identified (see subsection 3.2.2, Figure 21). While the latter can be explained by the higher copy numbers of proteins that are involved in amino acid biosynthesis per se when yeast cells grow on glucose-containing medium, genes encoding proteins involved in the TCA cycle should be repressed by glucose (discussed in section 4.3). The fact that cross-linked proteins of the TCA cycle were identified in mitochondria of the rho ${ }^{\circ}$ strain could be explained by the lack of complexes of the ETC and the mitoribosome, improving the identifications of proteins belonging to other complexes. Proteins involved in the TCA cycle were shown to be organized in a supercomplex for 
efficient substrate channeling (similar to the ETC supercomplexes), termed 'metabolon', that was recently identified by chemical fixation and mass spectrometry ${ }^{282}$.

However, at a second glance, a fully assembled 'metabolon' could not be confirmed in this thesis under neither condition. Only cross-links between Aco1, the aconitase, and the isocitrate dehydrogenase, Idh1 and Idh2, that catalyze consecutive steps within the TCA cycle, might indicate the formation of a 'metabolon' (see subsection 3.2.2, Figure 21). Surprisingly, both the aconitase and the isocitrate dehydrogenase cross-linked to Ilv5, the ketol-acid reductoisomerase involved in the biosynthesis of the amino acids valine and isoleucine, indicating a crosstalk between metabolism and amino acid biosynthesis. However, Aco1 and Ilv5 fulfill a biological dual role. They are not only involved in metabolism or amino acid biosynthesis pathways but also in mitochondrial genome maintenance ${ }^{45-47}$. Besides the aconitase, also other proteins of the TCA cycle are supposed to be involved at least in DNA/RNA-binding, such as the isocitrate dehydrogenase and the $\alpha$-ketoglutarate dehydrogenase ${ }^{45,416}$. Aco1, Idh2 and Ilv5 crosslinked to Mmf1 which is also a protein with a dual role. While on the one hand Mmf1 is involved in the Ile/Val biosynthesis pathway such as Ilv5, on the other hand it also participates in genome maintenance ${ }^{283,382}$. In summary, the cross-links between Aco1, Idh1 and Idh2, Ilv5 and Mmf1 identified in the $r h o^{\circ}$ data set might rather indicate an interplay between these proteins in their role in genome maintenance than a crosstalk between metabolism and amino acid synthesis. These interactions were not identified in the wild type mitochondria grown on either glycerol- or glucose-containing medium. While proteins that are involved in genome maintenance are undoubtedly active in wild type mitochondria such as Abf $2^{36,417}$, the genome maintenance function of the above described proteins with a dual role might be triggered when mtDNA is damaged. Why the described cross-links between proteins involved in genome maintenance were identified in a yeast strain that should completely lack mtDNA still needs to be investigated. A possible explanation for this might be that $r h 0^{\circ}$ strains are usually generated by the addition of ethidium bromide (see subsection 2.2.2). Damaged mtDNA or fragments of it might still be present inducing the recruitment of proteins that are involved in genome maintenance in mitochondria.

\subsubsection{TOM/TIM complexes}

One goal of cross-linking mitochondria derived from $r o^{\circ}$ yeast cells was to gain deeper insights into interactions of proteins belonging to the translocases of outer and inner membrane (TOM/TIM). $99 \%$ of the mitochondrial proteins are encoded by genes located in the nuclear DNA ${ }^{32}$. The TOM/TIM complexes aid the import and the translocation of 
these proteins and consist of at least 27 proteins $^{21}$ (see Supplementary Table 7). 19 and 21 of them were included in the databases for the cross-linked peptides search in the glycerol and glucose data set, respectively, and 17 of them in the databases for the $r o^{\circ}$ data set. In all data sets the number of identified cross-links within and between the TOM/TIM complexes was equally low (see subsection 3.2.3, Figure 22). The rho data set provided the highest number of CSMs in these complexes. In total, cross-links within and between eleven proteins of the TOM/TIM complexes were identified, seven of them were involved in interprotein cross-links. In all data sets, the highest number of cross-links was identified in the presequence translocase-associated motor (PAM) complex, part of the TIM23 complex that is located in the IM. The PAM complex shows ATPase motor activity and aids import of proteins into the matrix ${ }^{418}$. The PAM complex consists of soluble matrix proteins (Ssc1, Mge1) and proteins tethered to the IM, but which harbor large domains reaching into the matrix (Pam16, Pam18, Tim44). Therefore, these subunits are accessible for the cross-linker. Similarly, cross-links between Tim9 and Tim10, small TIM proteins that form a soluble complex within the IMS ${ }^{419}$, were also identified in every data set. All other proteins that belong to the TOM complex, the TIM23 complex and the TIM22 complex are integral membrane proteins of the OM and IM or - in case of the TOM complex - contain domains that protrude into the cytosol. Cross-links within some of these transmembrane subunits could be detected, such as Tom40 and Tom20 of the TOM complex, Tim50 of the TIM23 complex or Tim22 of the TIM22 complex, but only intraprotein cross-links within these proteins could be identified and no expanded protein-protein cross-linking network to other subunits of the respective complexes. Recently, the 3D structure of the yeast TOM complex could be solved, including the channel-forming subunit Tom40, the receptor subunit Tom22 and the regulatory subunits Tom5, Tom6 and Tom $7^{52}$. While Tom40 forms a $\beta$-barrel structure, the other proteins represent $\alpha$-helices attached to Tom40. Close inspection of the putative cross-linkable and digestible lysine and arginine residues revealed a major bottleneck of cross-linking small transmembrane proteins. In case of Tom5, Tom6 and Tom22 putative cross-linkable lysine residues that are located close to Tom40 are rare. Still, these residues are in cross-linkable distance to other subunits. However, upon cross-linking, these lysine residues are no longer susceptible to trypsin digestion. Only Tom22 harbors an arginine residue as cleavage site for trypsin, but cleavage would generate a long cross-linked peptide which might escape detection by XLMS. For transmembrane proteins with a higher molecular weight such as Tom40, in turn, especially arginine residues are favorably distributed in terms of potential tryptic cleavage sites, even if lysine residues were cross-linked. These caveats of cross-linking and digestion of transmembrane proteins described for the TOM complex can also be adapted to the TIM22 and TIM23 complexes, thus, explaining the low number of identified cross- 
links in the TOM/TIM complexes. In general, due to insufficient digestion, low abundance, and decreased solubility, the mass spectrometric analysis of membrane proteins remains challenging ${ }^{326}$. This is in line with the cross-linking study on yeast mitochondria performed by Makepeace et al. ${ }^{102}$ Although extracting mitochondrial proteins into a soluble and a membrane fraction, they could only identify two unique cross-links between proteins of the TOM complex and four unique cross-links between TIM proteins. The use of different endoproteinases such as chymotrypsin that cleaves C-terminally of aromatic amino acids, leucine, and methionine might improve the identification of membrane proteins.

Furthermore, cross-links between Tom40 and Por1 were identified. Por1 is discussed in other biological context in (sub)sections 4.2, 4.4.6, and 4.4.7. Por1 and Tom40 are functionally connected. Recently, the laboratories of Professor Nikolaus Pfanner and Professor Toshiya Endo independently pointed out an interaction of Por1 to Tom22, a subunit of the TOM complex ${ }^{420,421}$. They reported a dual role of Por1 and its participation not only in transporting metabolites but also in protein import by regulating the protein composition of the TOM complex. The TOM complex can form a dimeric or a trimeric structure ${ }^{422,423}$. The interaction between Por1 and Tom22 regulates Tom22's insertion to the TOM complex inducing the formation of the trimeric structure. The crosslinks observed in this thesis between Por1 and Tom40 of the TOM complex indicate that they are in close proximity to each other and corroborate their biologically relevant interaction. Tom40 cross-linked with its flexible $\mathrm{N}$-terminus to the $\mathrm{N}$-terminus of Por1 most likely on cytosolic side. In the $r 0^{\circ}$ strain, additionally a cross-link between Por 1 and Tom5 could be identified. Here, the $\mathrm{N}$-terminus of Tom5 cross-linked to the $\mathrm{N}$-terminal segment of Por1, also most likely on cytosolic side.

\subsection{Quantitative XL-MS approach}

The qualitative cross-linking data sets discussed above provided a general overview of protein-protein cross-linking networks in mitochondria from yeast grown on either glycerolor glucose-containing medium. The comparison between both networks was mainly based on counts of CSMs or the numbers of unique residue-to-residue cross-links. Normalization of the qualitative data sets was performed on the experimental level by cross-linking the same protein amount of each condition with the same amount of cross-linker. The qualitative analysis is prone to random errors, since observed differences in the crosslinking patterns might be a result of differences in the downstream workflow after the crosslinking reaction. This might be different lysis efficiency, different protein and peptide recovery after precipitation and digestion to the point of variations in the performance of the mass spectrometer. To gain more accurate insights into the differences of these two 
protein-protein cross-linking networks, a quantitative XL-MS approach was performed by applying a stable-isotopically labeled cross-linker. Mitochondria isolated from yeast grown on glycerol- or glucose-containing medium were either cross-linked with non-labeled BS3 or with isotopically labeled BS3-d4 and vice versa. Samples were then equally mixed based on the protein amount and the described workflow was applied (see subsection 3.1.2, Figure 6). Random errors then apply to both samples in equal measure. The final quantitation of residue-to-residue cross-links occurred on MS1 level by integrating the area of the peaks resulting from the non-labeled and the labeled peptides in the same run. Calculating the ratios of the specific areas resulted in a relative quantitation of residue-toresidue cross-links.

\subsubsection{Challenges of quantitative XL-MS}

Combining label-aided quantitation with XL-MS possesses a few challenges. One of them is the huge number of intraprotein cross-links within the residue-to-residue cross-links that are significantly more abundant than interprotein cross-links (see subsection 4.6.2). There are three explanations for this: (i) The introduction of a label and the subsequent mixing of the samples increase the sample redundancy. One example: A protein is digested into ten peptides. The mass spectrometer is fast enough to select all ten peptides for fragmentation in one duty cycle of a defined time period. In a labeling approach, this protein and its peptides are present in two versions, unlabeled and labeled. Hence, more precursors are present to be selected for fragmentation by the mass spectrometer, especially when extrapolating this to the whole mitochondrial proteome. This might result in a selection bias for high abundant peptides that most likely represent intraprotein cross-links. Less abundant interprotein cross-links that might have been identified in an unlabeled approach because the mass spectrometer can spend more time on their selection, might have been missed in the labeling approach. (ii) Also for the quantitative approach, the obtained spectra were searched against a database containing the 400 most abundant proteins for cross-linked peptides identification (discussed in detail in section 4.3). The difference compared to the qualitative data set is that the used database for the quantitative data set contains the most abundant proteins of both the glycerol and the glucose condition, while the number of entries (400 proteins) remained constant. The resulting database is even more biased towards high abundant proteins than the database used for the qualitative approach. (iii) The probability for an intraprotein cross-link to form within one protein is higher than for an interprotein cross-link to form between two different proteins. This in combination with a database containing high abundant proteins and the increased sample redundancy are possible reasons for the high number of intraprotein cross-links. A similar 
bias towards intraprotein cross-links was also observed in a quantitative XL-MS study on HeLa cells ${ }^{208}$. However, also differences in intraprotein cross-links can provide valuable information on conformational changes of protein regions ${ }^{232}$.

Another challenge are the so-called missing values. Missing values occur if a peptide(-pair) or its labeled counterpart is not identified among all replicates, increasing the uncertainty of the quantitation. This is due to the stochastic nature of data-dependent acquisition $^{163}$ (see also subsection 4.4.1) combined with the low yield of cross-linked peptides per $\mathrm{se}^{213}$ (see subsection 4.8.1). In this data set, $41 \%$ of unique residue-toresidue cross-links were identified in minimum three out of four replicates. With respect to interprotein residue-to-residue cross-links, only $16 \%$ were quantified in at least three replicates what further shifts the bias towards intraprotein cross-links. Therefore, the quantitative data sets were not as stringently filtered as the qualitative data sets, i.e. no CSM- and score-filtering was applied (see subsection 4.4.1). This would have increased the number of missing values. The significance of the data was calculated by a one-sample $t$ test on median-normalized data (see subsection 2.4.4). Other labeling strategies are discussed in subsection 4.6.3.

\subsubsection{Biological interpretation of the quantitative data}

The obtained quantitative cross-linking data are in agreement with the qualitative data. Also in the quantitative data set, the general trend is observable that cross-links showing higher abundances in one or the other condition are between proteins that have a higher copy number in one of the conditions per se. Most of the cross-links are intraprotein crosslinks ( $79 \%$ and $93 \%$ in the glycerol and glucose condition, respectively). The increased intensity induced by the different carbon sources of many of these intraprotein cross-links can be explained by the higher copy numbers of the involved proteins ${ }^{72}$ (see subsection 3.1.6, Figure 13). Especially in the glycerol condition, 45 out of 46 proteins whose residueto-residue cross-links were significantly increased showed also higher copy numbers in that specific condition. This, in turn, can be explained in most cases by glucose repression (see (sub)sections 4.3 and 4.4.5). For example, a high number of intraprotein cross-links within the mitochondrial aldehyde dehydrogenase Ald4 and also within the glycerol 3phosphate dehydrogenase Gut2 were identified. Both these proteins are involved in glycerol- and pyruvate-metabolism in yeast cells grown on glycerol-containing medium and the respective genes are repressed by glucose (see subsection 4.4.5). Other intraprotein and interprotein cross-links that showed higher intensities under glycerol condition belong mainly to the OXPHOS system, especially CV, and the TCA cycle - complexes whose proteins also have higher copy numbers under glycerol condition. Interestingly, also cross- 
links between Om45 and Nde1 showed higher intensities under glycerol condition (see subsection 4.4.6).

The quantitative results reflecting the cross-links with higher abundance in the glucose condition are more diverse. Besides the intraprotein and interprotein cross-links within or between proteins whose copy numbers are higher in the glucose condition such as proteins belonging to the amino acid biosynthesis pathways (e.g. Lys12, Ilv3, Lys4, Bat1; see (sub)sections 3.4, 4.3, and 4.4.3), there are several cross-links within and between proteins that showed similar copy numbers under both conditions. The most prominent example, the pyruvate dehydrogenase complex (PDH) was discussed in detail in subsection 4.4.5. Surprisingly, also Por1, the voltage-dependent anion channel (VDAC) located in the OM, showed higher intensities of intraprotein cross-links in the glucose condition, although the copy number of Por 1 is higher in the glycerol condition ${ }^{72}$. This might be explained by two different conformations of the channel that have been present in the moment of cross-linking. In 2008, three structures of VDAC1 were resolved by NMR (human VDAC1 $)^{424,425}$ and X-ray crystallography (murine VDAC1) ${ }^{403}$. Unfortunately, the structure of yeast VDAC1 - Por1 - has not been resolved yet but shows a sequence similarity of $94 \%$ to the murine VDAC1. All these structures display a channel consisting of $19 \beta$-barrel sheets and an $\mathrm{N}$-terminal $\alpha$-helix formed by the first $20-25$ amino acids. It was proposed that the $\mathrm{N}$-terminal segment is not part of the membrane-embedded ring structure but is located in the lumen of the channel where it might be involved in the gating mechanism. Indeed, the more abundant intraprotein cross-links identified in the glucose condition are within the first 95 amino acids, covering the $\mathrm{N}$-terminal $\alpha$-helix and the first five $\beta$-sheets. According to the structures, this might indicate a closed conformation of VDAC1. In an open conformation, the $\alpha$-helix would be outside the channel lumen or at least move more into the direction of $\beta$-sheets $15-18^{403}$ to enable the transport of metabolites such as ATP and ADP, and redox equivalents. Unfortunately, no cross-links that are higher abundant in the glycerol-condition could be identified to corroborate this hypothesis. It has been shown that a por mutant yeast strain is viable but respiratorydeficient ${ }^{426}$. This result emphasizes the importance of Por 1 in respiration and explains its higher copy number in yeast cells grown on glycerol-containing medium. Therefore, it might be more likely to cross-link Por1 in an open conformation in mitochondria isolated from yeast grown on glycerol-containing medium than in mitochondria isolated from yeast grown on glucose-containing medium. However, Professor Marco Colombini raised doubts about the correctness of the published structures ${ }^{427,428}$. He addressed the point that biochemical data do not fit with a $19 \beta$-barrel structure, but rather with a $13 \beta$-barrel structure with a membrane-integrated $\alpha$-helix. Against this background, the interpretation of the observed abundances of Por1's intraprotein cross-links is even more difficult. 


\subsubsection{Other labeling strategies in combination with XL-MS}

Instead of using isotopically labeled cross-linkers, the laboratory of Professor James Bruce combined metabolic SILAC labeling with XL-MS in studies of various complex samples. They analyzed differences in protein interaction patterns of Hsp90 and the effects of smallmolecule inhibitors ${ }^{429}$, in multidrug-resistant human carcinoma cells ${ }^{208}$ and in mitotic inhibitor-treated human cells ${ }^{430}$ while simultaneously gaining information about relative protein abundances. Several SILAC labeling protocols exist also for yeast strains ${ }^{431}$ and could have been used in this thesis as well. However, since this thesis deals exclusively with mitochondria, a SILAC study would have been disproportionately expensive. In this regard, the use of a labeled cross-linker is more straightforward and flexible, especially for systems where SILAC labeling is not applicable. One example for this is described in the $\mathrm{PhD}$ thesis of Dr. Iwan Parfentev, Max Planck Institute for Biophysical Chemistry, Göttingen, with the title 'Elucidation of protein interactions in complex samples by proteinprotein cross-linking of synaptosomes' (SUB Göttingen-Zentralbibliothek, restricted access until August 08, 2020). Dr. Iwan Parfentev used a labeled cross-linker to quantify changes of cross-linking patterns within resting and excited synaptosomes isolated from rat brains. SILAC experiments are also practicable with animals, e.g. labeled mice ${ }^{172}$, but prohibitively expensive if only one part of the animal is used.

The laboratory of Professor Lan Huang combined chemical TMT labeling with cross-linking ${ }^{233}$. They cross-linked cytochrome $c$ with the MS-cleavable cross-linker DSSO (see subsection 4.8.2) and subsequently labeled peptides with TMT after protein digestion. Regardless of the used cross-linker, this approach enables accurate quantitation of crosslinked peptides across multiple conditions. Although very beneficial, this approach still needs to be evaluated for more complex samples and larger batches. Recently, it was shown that TMT labeling of a large number of multiplexed samples introduces batch effects and an inflation of missing values ${ }^{432}$. Besides that, for accurate quantitation, an advanced tribrid mass spectrometer with MS3-capability is beneficial. TMT labeling is also performed at a late stage in the sample preparation workflow, and, hence, does not account for random errors occurring in previous steps ${ }^{170}$, e.g. during digestion and desalting. Related to that, using labeled cross-linkers as in this thesis can be seen as chemical labeling on protein level accounting for random errors, at least starting from the lysis.

Label-free approaches to quantify cross-linked peptides were introduced by Müller et al..$^{234,235}$ By cross-linking bovine serum albumin the authors could demonstrate the reproducibility and accuracy of quantifying cross-linked peptides on MS1 level via datadependent acquisition (DDA) ${ }^{234}$. The authors also set up a data-independent acquisition 
(DIA) strategy ${ }^{235}$. During DIA, precursors are not selected for fragmentation based on their intensity but all precursors in defined $m / z$ windows will be selected for fragmentation ${ }^{162,163}$. Quantitation is then preferably performed on MS2 level resulting in an increase of accuracy and therefore in decreased coefficients of variation (CV) among replicates ${ }^{161,163}$ even in cross-linked protein complexes ${ }^{235}$. However, after spiking cross-linked proteins into a noncross-linked $E$. coli lysate, the CV values of quantified cross-linked peptides were increased due to precursor interferences ${ }^{235}$. Since all precursors are fragmented in combination with a cross-linker, resulting spectra are highly chimeric and need to be evaluated very carefully. Label-free quantitation is the most error-prone strategy of the ones introduced in this subsection.

\subsection{Cross-linking of human mitoplasts with two different cross-linkers}

The cross-linking workflow that was established for yeast mitochondria was also applied to human mitoplasts with a few changes. In this section, the experimental rationale is emphasized, and results are discussed. Furthermore, it is also highlighted how the use of cross-linkers with different reaction chemistries provide complementary results by addressing different regions of proteins ${ }^{433,434}$.

\subsubsection{General comments on the experimental rationale}

Mitoplasts are mitochondria without an outer membrane and intermembrane space but with an intact inner membrane and matrix compartment. Human mitoplasts instead of fully functional mitochondria were cross-linked to reduce the sample complexity, hence, increasing the analytical depth for protein-protein cross-links within the IM and the matrix. Accordingly, the majority of cross-links were identified between proteins localized either to the IM or to the matrix (see section 3.6, Figure 30).

Instead of using the software pLink 1 in combination with a restricted database for cross-linked peptides identification, searches were performed by the successor software pLink $2^{258}$ which was published in 2018. Databases comprising all proteins that were identified in the sample based on their linear peptides were generated and used for the search (see subsection 2.4.2.1). pLink 2 uses an optimized algorithm for the analysis decreasing the analysis time by the factor 40 in comparison to pLink $1^{258}$. Therefore, searches against databases including more than 1000 proteins can be afforded in a feasible amount of time. For Homo sapiens, a regularly updated and curated list of mitochondrial proteins is available, the MitoCarta2.0435. This list comprises 1158 genes encoding human mitochondrial proteins so far (April 2020). In the beginning of the project 
it was discussed to use the MitoCarta2.0 as a database for the search of cross-linked peptides. However, so far undetected mitochondrial proteins or proteins whose exact location is still unclear would have been excluded. By including all identified proteins (approximately 1500 for the BS3 and 1900 for the EDC data set) also low abundant ones were part of the databases. A drawback is that also curated non-mitochondrial proteins such as histones were included in the database and found to be cross-linked most likely due to impurities during mitochondria isolation.

\subsubsection{Cross-linkers with different reaction chemistries}

Human mitoplasts were cross-linked with BS3 or EDC. While BS3 is a lysine-to-lysine cross-linker, EDC connects lysine residues with aspartate or glutamate residues. The two cross-linkers differ not only in their reaction chemistry, but also in spacer length. BS3 possesses a spacer with a length of $11.4 \AA$ resulting in a distance of approximately $30 \AA$ between $\mathrm{C} \alpha$-atoms of lysine residues, accounting for flexibility as calculated by a molecular dynamics simulation study ${ }^{187}$. EDC is a zero-length cross-linker imposing an average distance of approximately $14 \AA$ between Ca-atoms. The obtained cross-linking data are complementary to each other. 40 unique protein-protein cross-links were identified after BS3-cross-linking, 39 after EDC-cross-linking (see subsection 3.6.3, Figure 30). Only 5 of the protein-protein cross-links could be identified in both data sets indicating that different proteins and protein regions are addressed by these two cross-linkers. This is underlined by the tendency that EDC favors proteins with a slightly lower isoelectric point ( $\mathrm{pl}$ ) than BS3 that, in turn, cross-linked proteins with slightly higher pl values (see subsection 3.6.1, Figure 29). This can be explained by the different reaction chemistries of the two crosslinkers. EDC cross-links lysine residues to acidic residues like aspartate and glutamate that both have lower $\mathrm{pK}_{\mathrm{a}}$ values than lysine residues. However, the low overlap might also have been caused by the stochastic nature of data-dependent acquisition (see subsections 4.4.1 and 4.6.1).

Although the numbers of involved proteins and unique residue-to-residue crosslinks were higher in the BS3 data set, relatively more interprotein cross-links could be identified in the EDC data set (filtered, see subsection 3.6.1, Table 9). These findings have also been reported by Gutierrez et al. ${ }^{434}$ who cross-linked the human COP9 signalosome separately with three MS-cleavable cross-linkers with different reaction chemistries: DSSO $(\mathrm{K}-\mathrm{K})$, DHSO (D/E-D/E) and BMSO (C-C). First, the authors could also observe that crosslinkers with different chemistries increase the comprehensiveness of a protein-protein cross-linking network and that the obtained data are complementary to each other. Second, the authors also reported the highest number of identified cross-links for DSSO 
$(\mathrm{K}-\mathrm{K})$, but $\mathrm{DHSO}(\mathrm{D} / \mathrm{E}-\mathrm{D} / \mathrm{E})$ yielded more interprotein cross-links (in this regard, BMSO even outperformed DHSO). Similar trends were observed by Kim et al. ${ }^{433}$ who cross-linked the yeast nuclear pore complex with DSS (K-K) and EDC (K-D/E). Salt bridges formed between lysine residues and aspartate or glutamate residues that are well-distributed on protein surfaces and interaction interfaces stabilize protein interactions ${ }^{434,436-438}$. Against this background it is reasonable that, especially in case of the zero-length cross-linker EDC, a cross-link between a lysine residue and an acidic residue such as aspartate and glutamate occurs preferentially within interaction interfaces between two distinct proteins resulting in the identification of an interprotein cross-link.

\subsubsection{Intraprotein cross-links outnumbered interprotein cross-links}

The relative number of intraprotein versus interprotein cross-links on protein level was much higher for human mitoplasts than for yeast mitochondria. In the BS3 data set, $86 \%$ of the identified protein-protein cross-links represent intraprotein cross-links and $14 \%$ interprotein cross-links. In the EDC data set, $80 \%$ of the identified protein-protein crosslinks represent intraprotein cross-links and $20 \%$ interprotein cross-links. The increased relative number of interprotein cross-links after EDC cross-linking compared with BS3 cross-linking is in line with the results discussed in subsection 4.7.2. However, in comparison with the yeast data set in which the ratio of intraprotein to interprotein crosslinks is $\sim 60 \%$ to $\sim 40 \%$ on protein level, the human data sets provide much more intraprotein cross-links. Other XL-MS studies dealing with murine and yeast mitochondria also showed a higher number of interprotein cross-links (see subsection 4.4.8, Table 11). Surprisingly, in the XL-MS study on human mitochondria by Ryl et al. ${ }^{101}$ a similar tendency towards intraprotein cross-links was observed, similar to this thesis. The authors reported $90 \%$ intraprotein and $10 \%$ interprotein cross-links on protein level after cross-linking human mitochondria with the non-cleavable cross-linker DSS (a derivative of BS3 without the charged sulfo-groups). Ryl et al. constituted the high number of intraprotein versus interprotein cross-links by different FDR calculations. In their study, FDR calculation was applied separately on intraprotein and interprotein cross-links and not globally like in all other mitochondrial XL-MS studies. Indeed, this different strategy for FDR calculation reduces the number of interprotein cross-links justified by the higher accuracy of FDR calculation ${ }^{101}$. When Ryl et al. applied a global FDR calculation to their data set, they could also increase the ratio of interprotein cross-links to $16 \%$. However, this number is still lower compared to the other mitochondrial XL-MS studies, indicating that the FDR calculation cannot be the only reason for the decreased number of interprotein cross-links. To exclude a bias towards intraprotein cross-links induced by different software suites or 
database sizes, the data set of cross-linked mitochondria isolated from yeast grown on glycerol-containing medium obtained in this thesis was reanalyzed with pLink 2 applying the same settings as for the human mitoplasts data sets and with a database containing all identified proteins. This resulted in $\sim 70 \%$ intraprotein and $\sim 30 \%$ interprotein crosslinks on protein level, similar to all other non-human mitochondrial XL-MS studies. This indicates that biases induced by different software and database sizes can be excluded. Keller et al. ${ }^{439}$ predicted theoretical interprotein cross-link fractions in comparison to intraprotein cross-links by analyzing data sets of cross-linked cells and by mapping the cross-links to available 3D structures. The authors could demonstrate that the interprotein cross-link fraction is significantly higher for "in vivo" cross-linked HeLa cells compared to a cross-linked lysate of the same cell line, i.e. more proteins are assembled into complexes under native conditions than after lysis. While this result can be transferred to human mitoplasts, where protein complexes might have been disrupted due to digitonin treatment leading to less interprotein cross-links, cross-linking results from human mitochondria obtained by Ryl et al. need further evaluation to explain the low number of interprotein cross-links. A general explanation could be the higher volume of human mitochondria $\left(0.26 \mu \mathrm{m}^{3}\right.$ in HeLa cells $\left.{ }^{440}\right)$ compared to yeast mitochondria $\left(0.14-0.16 \mu \mathrm{m}^{3}\right.$ in diploid and haploid budding yeast, respectively ${ }^{441}$ ) leading to a stronger dilution of the cross-linker. However, a potential correlation between mitochondrial volume and the number of identified interprotein cross-links cannot be confirmed for rodent mitochondria $\left(0.27 \mu \mathrm{m}^{3}\right.$ in rats $\left.{ }^{442}\right)$.

\subsubsection{Biological interpretation of the identified protein-protein cross-links}

Based on the successful application of BS3 to cross-link proteins located in all subcompartments of yeast mitochondria, this cross-linker was also used to cross-link human mitoplasts. EDC, also a water-soluble cross-linker similar to BS3, was considered to cross-link proteins in all mitochondrial subcompartments as well. Accordingly, both cross-linkers could pass through the IM of human mitoplasts (see subsection 3.6.3, Figure 30 and discussion about BS3's ability to pass through mitochondrial membranes in section 4.2 that can also be applied to EDC). As discussed in subsection 4.7.3, the number of intraprotein cross-links outnumbered interprotein cross-links for both cross-linkers. Among the proteins that provide the most intraprotein cross-links is, for example, the chaperone HSPD1/HSPE1. It comprises $19 \%$ and $26 \%$ of all CSMs in the BS3 and the EDC data set, respectively. The chaperone is a soluble matrix protein and among the ten most abundant proteins based on the identifications of the linear peptides (data not shown). Additionally, $23 \%$ of its amino acid composition comprises $\mathrm{K}, \mathrm{D}$ and $\mathrm{E}$ which is above 
average (referring to an amino acid composition calculation of a set of globular proteins that calculated the average sum of $\mathrm{K}, \mathrm{D}$ and $\mathrm{E}$ within these proteins to $18 \%{ }^{443}$ ). The combination of high abundance, solubility and amino acid composition makes this chaperone a perfect candidate for BS3 and EDC cross-linking. The high number of intraprotein cross-links to this chaperone was also confirmed by Ryl et al. ${ }^{101}$ Besides the high number of intraprotein cross-links, 79 interprotein cross-links defining unique proteinprotein cross-links could be identified in total. Some of them will be discussed in the following. Like in yeast mitochondria, also the OXPHOS system in human mitoplasts was involved in cross-linking reactions. In contrast to yeast, mammalian mitochondria harbor a proton pumping $\mathrm{Cl}^{444}$. Human $\mathrm{Cl}$ consists of 45 subunits forming an L-like structure with one arm embedded in the IM and the other arm protruding into the matrix ${ }^{445}$. Eleven of these subunits formed interprotein cross-links, seven of the subunits belong to the soluble matrix-arm. The tendency to cross-link soluble protein domains is in agreement with the so far presented results and with the fact that membrane-embedded proteins are difficult to analyze with MS (see subsection 4.5.2). The assembly of $\mathrm{Cl}$ is aided by assembly factors ${ }^{446,447}$. In this thesis, two recently identified assembly factors of human $\mathrm{Cl}$ could be identified in the EDC data set, namely TIMMDC1 and DMAC10. TIMMDC1 cross-linked to NDUFA8, part of the V-module of the membrane-arm of $\mathrm{Cl}$. In an interaction proteomics study performed by Guarani et al. ${ }^{448}$, TIMMDC1 was shown to associate with $\mathrm{Cl}$ and the $\mathrm{Cl}$ assembly factor complex MCIA. The authors could also show that depletion of TIMMDC1 led to significantly reduced $\mathrm{Cl}$ activity and an accumulation of $\mathrm{Cl}$ assembly intermediates. This was also found by Andrews et al. ${ }^{449}$ who identified NDUFA8 among the proteins that interact with TIMMDC1 by performing co-immunoprecipitation in combination with MS. Andrews et al. proposed a model in that NDUFA8 and TIMMDC1 are assembled into the same $\mathrm{Cl}$ assembly intermediate. This is corroborated by the identified cross-link in this thesis. DMAC10 cross-linked to NDUFB10, also part of the membrane-arm of CI. In a study performed by Stroud et al. ${ }^{450}$, knockout of DMAC1 led to $\mathrm{Cl}$ assembly defects. In a pull-down experiment, the authors demonstrated that DMAC1 interacts with proteins of the ND4-module of $\mathrm{Cl}$ of which NDUFB10 is part of. While the exact function of TIMMDC1 and DMAC1 still remains elusive, the identified cross-links corroborate the hypothesis that these proteins are involved in the assembly of $\mathrm{Cl}$. Another cross-link of $\mathrm{Cl}$ was identified in the BS3 data set. The cross-link between NDUB4, a protein of $\mathrm{CI}$, to UQCR10, a protein of CIII, provided evidence for an ETC supercomplex formation in human mitoplasts. While ETC supercomplex formation in yeast was discussed in detail in subsection 4.4.4, evidence for supercomplex formation also in mammals was provided long time ago ${ }^{87,89,94}$. Recently, a structure for the human $\mathrm{CICIII}_{2} \mathrm{CIV}$ supercomplex and even a megacomplex structure $\mathrm{Cl}_{2} \mathrm{CIII}_{2} \mathrm{CIV}_{2}$ was proposed ${ }^{90}$. Since the cross-linking 
data in regard to interprotein cross-links for human mitoplasts are very sparse, unfortunately no further evidence could be provided for one of the proposed structures but only for the close proximity of $\mathrm{Cl}$ and $\mathrm{CIII}$. The identified cross-link between $\mathrm{Cl}$ and $\mathrm{CIII}$ induced by BS3 satisfies the distance constraint of $30 \AA$ (see subsection 3.6.3, Figure 31). Also Ryl et al. ${ }^{101}$ did not identify comprehensive interprotein cross-links within the $\mathrm{CICIII}_{2} \mathrm{CIV}$ supercomplex. While no cross-links between $\mathrm{Cl}$ and $\mathrm{CIII}$ were identified by the authors, they, in turn, could identify cross-links between CIII and CIV.

Cross-links between ROMO1 and PHB (EDC) as well as PHB2 (BS3) were identified in this thesis. Prohibitin exists in two homologs, PHB and PHB2, forming an approximately two megadalton large complex located in the IM with proposed functions in protein folding 451 and maintenance of mitochondrial morphology 452 . Prohibitins are transported into mitochondria and translocated via the TIM23 complex ${ }^{453}$. Remarkably, ROMO1 was recently described as a novel constituent of the TIM23 complex by Richter et al. ${ }^{299}$ Furthermore, both prohibitin homologs cross-linked to OCIAD1 (EDC: PHB; BS3: PHB2), a protein which was found to interact with PHB2 by Richter-Dennerlein et al. ${ }^{300}$ It was also shown recently that OCIAD1 interacts with $\mathrm{Cl}$ and regulates energy metabolism in human pluripotent stem cells ${ }^{454}$. However, a large-scale study on the human mitochondrial interactome by applying BiolD suggests dual localization of OCIAD1 in mitochondria as well as peroxisomes ${ }^{455}$ (Antonicka et al., bioRxiv, April 2020).

\subsection{Other cross-linking and analysis workflows}

$\mathrm{XL}-\mathrm{MS}$ is no longer restricted to the interrogation of interactions within purified protein complexes but expands its capabilities to analyze more complex samples and, ultimately, to identify large protein-protein cross-linking networks in organelles or cells. To achieve this goal, a reliable identification of cross-links is essential. Several strategies to enrich cross-linked peptides and to reduce the search space are becoming increasingly popular.

\subsubsection{Enrichment of cross-linked peptides to increase their identification rate}

The cross-linking reaction efficiency is estimated to be $1-5 \%{ }^{213}$. Therefore, non-crosslinked, i.e. linear, peptides greatly outnumber cross-linked peptides and it is necessary to enrich for cross-linked species prior to LC-MS/MS analysis. An increased sensitivity for cross-linked peptides will improve spectrum quality and, hence, the reliable identification of these peptides. Two approaches that take advantage of peptide properties are commonly used in the XL-MS community ${ }^{183}$, namely the enrichment by peptide size 
exclusion chromatography (peptide SEC) ${ }^{214}$ and by strong cation exchange chromatography $(\text { SCX })^{196,215,456}$. Peptide SEC, also used in this thesis, is based on the molecular weight. Cross-linked peptides are longer and bulkier as linear peptides, thus having a higher mass. Most of the spectra obtained during the course of this thesis were identified in the early fractions after peptide SEC. For example, in the first biological replicate of cross-linked mitochondria derived from yeast grown on glycerol-containing medium, $93 \%$ of all spectra were identified in the first five fractions out of ten. Enrichment by SCX is based on an increase in the peptide net charge. Cross-linked peptides possess more positive charges than linear peptides, at least two positively charged $\mathrm{N}$-termini as well as two positively charged lysine or arginine residues due to trypsin digestion. Accordingly, cross-linked peptide species will elute in the end of the gradient with higher salt concentrations that is used for elution. Due to the high salt concentrations in the SCXfractions containing the cross-linked peptides, an additional desalting step prior to LCMS/MS analysis is necessary. A combination of SCX and C18 material (mixed-mode cartridges) $)^{457}$ as well as two-diagonal SCX (ChaFRADIC) ${ }^{458}$ were also applied to protein complexes and improved the identification of cross-linked peptides. These combined approaches still need to be evaluated for more complex samples.

Another strategy to enrich for cross-linked peptides is to use trifunctional, enrichable cross-linkers. Here, in addition to the two reactive groups, an affinity tag such as biotin or an option for click-chemistry is synthesized to the cross-linker206,216,217,459. Recently developed cross-linkers also take advantage of phosphor-based tags ${ }^{213,219}$, suitable for immobilized metal affinity chromatography (IMAC) enrichment. These tags enable targeted enrichment of cross-linked peptide species and increase the sensitivity of their identification. However, due to the additional tag the cross-linker becomes bulkier. This might increase steric hindrance during the cross-linking reaction. Although the enrichment of tagged cross-linkers adds another step in the sample preparation workflow, it could also supersede the need for subsequent strategies such as peptide SEC or SCX ${ }^{213}$.

\subsubsection{Reducing the search space}

A restricted database as a strategy to reduce the search space for the identification of cross-linked peptides was discussed in section 4.3. Another strategy is becoming increasingly popular: the application of MS-cleavable cross-linkers for the analysis of complex samples ${ }^{221-223}$. Due to the labile bond that can be cleaved in the gas phase of the mass spectrometer and the formation of reporter ions that can be selected for additional MS3 fragmentation, a reliable identification of cross-linked peptides can be performed. Ultimately, cross-linked peptides can be searched against databases covering whole 
proteomes, since the $n^{2}$ search space is reduced to $2 n$. However, to benefit from the MS3 fragmentation, a tribrid mass spectrometer containing a quadrupole, an orbitrap, and an ion trap is necessary. Furthermore, the MS3 scan costs time and will increase the duty cycle of the instrument. On the one hand, this comes along with a more reliable identification of cross-links. On the other hand, sensitivity will be sacrificed due to a prolonged duty cycle, i.e. the total number of cross-linked peptides identifications per sample will be reduced. Since the reporter ions are still identifiable also with higher collision energies, an MS3 approach is not mandatory. MS-cleavable cross-linkers can also be analyzed with an MS2 fragmentation only. Nevertheless, in some cases the labile bond within the cross-linker does not break efficiently which renders a successful identification challenging ${ }^{460}$. Since spectra that are generated after cross-linking with MS-cleavable cross-linkers are either obtained on MS3 level or contain specific reporter ions, special software suites have to be used for reliable peptide identification. Software that are capable of analyzing these data are for example XlinkX ${ }^{461}$, MeroX ${ }^{462}$ or MetaMorpheus ${ }^{460}$.

Cross-linkers cannot only be cleaved within the mass spectrometer, but also during the workflow beforehand. Parfentev et al. ${ }^{463}$ used a thiol-cleavable cross-linker to generate a peptide-focused database containing only the sequences of these peptides that were modified with a cross-linker remnant after reduction of the cross-linker. This approach led to a significant reduction in search time. However, the generation of such a peptidefocused database and the initial cross-linking experiment with a non-cleavable cross-linker are two separate steps in the workflow, resulting in additional work and measurement time. Reduction of the search space based on the knowledge about peptides' "cross-linkability" was already introduced by Lima and co-workers implemented in their own software suite SIM-XL ${ }^{464}$ and by Buncherd et al. ${ }^{465}$ in combination with a two-dimensional SCX fractionation strategy.

Another way of reducing the search space is by combining a mixture of a crosslinker with its stable-isotopically labeled counterpart and a suitable software. This was demonstrated by Rinner and colleagues and their software $x$ Quest $^{215}$ for large databases. The software performs a pre-search that detects cross-linked peptide pairs indicating a mass shift induced by the heavy label. Only these pairs are considered for the final crosslinked peptides database search, the others are treated as linear peptides. While this strategy represents a very reliable way for the identification of cross-linked peptides, the introduced heavy label will have a negative effect on the total identifications. The instrument does not only need time to fragment a single cross-linked peptide pair but also must select the labeled counterpart for a successful identification while the duty cycle remains constant (see also section 4.6). 


\subsection{Other strategies for interrogating protein-protein interactions using mass spectrometry}

Chemical cross-linking in combination with mass spectrometry is able to characterize protein-protein interactions in their native environment. Benefits are the localization of interaction partners within a radius of a few Ångström and the identification of the exact amino acid residues that participated in the cross-linking reaction. Cross-linkers with different spacer lengths, reaction chemistries and solubilities are commercially available and with the increasing sensitivities of mass spectrometers and the power of analysis software XL-MS becomes more and more a routine work, even for elucidating large protein-protein cross-linking networks in complex systems ${ }^{205,206,208,217}$.

Besides XL-MS, also other techniques exist that are capable of interrogating PPIs in combination with mass spectrometry. Approaches such as immunoprecipitation (IP) or affinity purification (AP) in combination with MS for protein identification are low-throughput methods. Their success is strongly based on the availability of specific antibodies in case of $\mathrm{IP}^{320}$. In case of AP, an introduced tag alters the molecular weight of a protein and might influence its natural behavior, and so its interaction partners. Also, the mild isolation conditions make it difficult to tackle membrane proteins ${ }^{466}$ and usually cause a strong background ${ }^{320}$.

Another approach that came up in the recent years is proximity labeling. Here, the protein of interest is fused to an enzyme that adds tags to proteins that are in close proximity. Via these tags, proteins can be purified and identified. In BiolD ${ }^{178}$, the bacterial biotin ligase BirA transfers biotin to lysine residues. In APEX ${ }^{179}$, the monomeric ascorbate peroxidase is used to attach biotin via a mechanism based on radical formation to tyrosine, cysteine, histidine and tryptophan residues. While the reaction catalyzed by APEX occurs in the range of milliseconds, biotin labeling by BioID takes up to 24 hours, one of its major drawbacks ${ }^{466}$. APEX, in turn, needs hydroxide peroxide which can induce cellular stress and activate pathways that would not have been activated in the natural environment. Both approaches cover an interaction distance of 10-20 nm and, hence, provide a much lower resolution than XL-MS. From this it can be concluded that labeled proteins do not necessarily interact directly with the protein of interest ${ }^{466}$. Also, for both BiolD and APEX a genetic protein engineering step is necessary and are therefore low-throughput methods $^{320}$. A strong benefit of these techniques over XL-MS is its suitability in living animals ${ }^{467}$.

Complexome profiling is especially used to analyze the composition of protein complexes in mitochondria ${ }^{468-470}$. Here, complexes are mildly solubilized and separated by blue native-gel electrophoresis (BN-PAGE). The gel is then cut into slices, proteins are 
extracted, and detected as well as quantified by LC-MS/MS. While this approach is very powerful in identifying complex intermediates, it is very laborious if the workflow is not automated. To draw reasonable conclusions from the label-free quantitative data, extensive protein correlation profiling (PCP) 471 needs to be carried out. Furthermore, ingel workflows are prone to sample loss and suffer from reduced reproducibility ${ }^{472}$. BNPAGE can be replaced by protein SEC that was successfully applied to HEK cells leading to the characterization of 462 complexes $^{473}$.

\subsection{Summary and perspective}

In this thesis, protein-protein cross-linking networks of mitochondria derived from Saccharomyces cerevisiae grown on a fermentable and a non-fermentable carbon source were elucidated by chemical cross-linking in combination with mass spectrometry (XLMS). For this, the non-cleavable cross-linker BS3 was applied and databases including the 400 most abundant proteins were used for cross-linked peptides searches. Networks were qualitatively compared to each other. In this context, it could be shown that observed differences strongly correlate with the energy metabolism of the yeast cells affected by the carbon source. Glycerol as non-fermentable carbon source is primarily metabolized via the oxidative phosphorylation (OXPHOS) system located in mitochondria, while glucose is mainly fermented for ATP production. Consequently, cross-links within the OXPHOS system and the TCA cycle showed higher numbers in mitochondria under glycerol condition. In this context, the participation of $\mathrm{Ndi1}$ in an $\mathrm{Ndi1}_{1} \mathrm{Cll}_{2} \mathrm{CIV}_{2}$ electron transport chain supercomplex was proposed. Furthermore, interactions of so far uncharacterized proteins could be revealed. Amongst them, cross-links between Min8 and Cox12 were identified. Ultimately, Min8's association with the cytochrome $c$ oxidase was substantiated by biochemical approaches and its localization within the complex was proposed by docking experiments. Additionally, a quantitative cross-linking mass spectrometry approach was successfully applied that corroborated also statistically the observed differences in yeast mitochondria originating from yeast grown on glycerol- or glucosecontaining medium. However, the significance of this approach was limited by major changes of protein copy numbers induced by the different carbon sources. Therefore, significantly changed residue-to-residue cross-links were dominated by intraprotein crosslinks. Mitochondria derived from a $r \mathrm{ho}^{\circ}$ yeast strain were cross-linked to identify interactions within complexes such as TOM/TIM, MICOS or interactions of low abundant proteins that were barely identified in the wild type strains. Unfortunately, respective results did not significantly improve. A protein-protein cross-linking network in human mitochondria derived from HEK cells was also elucidated by using two cross-linkers with 
different reaction chemistries. Similar to the quantitative approach applied to yeast mitochondria, the majority of identified cross-links in human mitochondria were intraprotein cross-links. Nonetheless, XL-MS emerges as a powerful tool in structural and systems biology. Detection of conformational changes within proteins or protein complexes and the elucidation of protein-protein interactions on a proteome-wide scale are supported by the development of mass spectrometers with increasing sensitivity, by cleavable cross-linkers addressing different amino acid residues, by the possibility to also track changes quantitatively, and by improving algorithms for the identification of cross-linked peptides. With respect to the analysis of mitochondria, the analysis depth has to be increased to also identify low abundant proteins and complexes such as TOM/TIM and MICOS. To achieve this, high concentrations of MS-cleavable cross-linkers can be applied to mitochondria to both increase the number of cross-links and to search against whole proteomes. This is important to also detect interactions between mitochondria and other organelles, such as the endoplasmic reticulum or peroxisomes. A quantitative XL-MS approach combining MS-cleavable cross-linkers and labeling via TMT could be used to interrogate structural changes between mitochondria of healthy humans and humans suffering mitochondrial diseases, e.g. induced by CIV deficiencies. In this case, changes within protein copy numbers should be minor and therefore should not influence the significance of the quantitative approach. 


\section{Appendix}

\subsection{Supplementary figures}

\author{
Glycerol_unfiltered \\ unique residue-to-residue cross-links
}

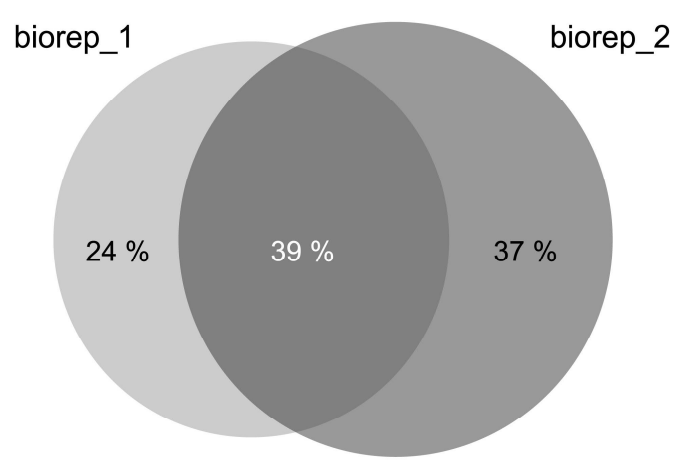

Glucose unfiltered

unique residue-to-residue cross-links

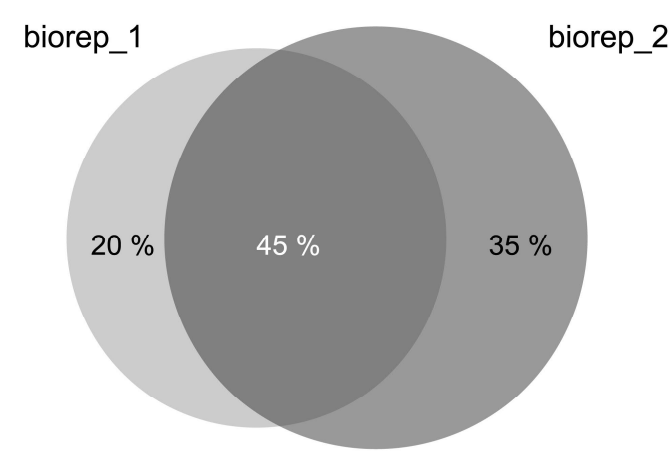

Glycerol_filtered

unique residue-to-residue cross-links

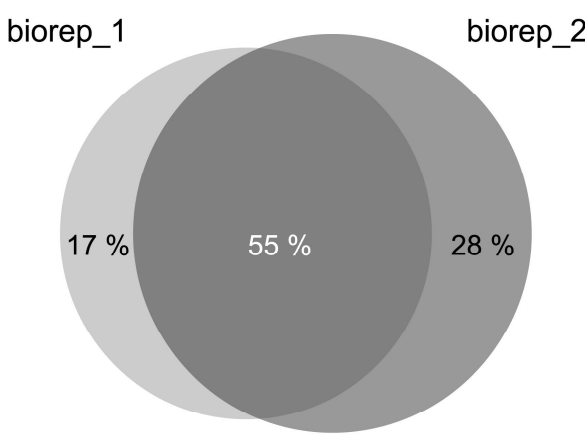

Glucose filtered unique residue-to-residue cross-links

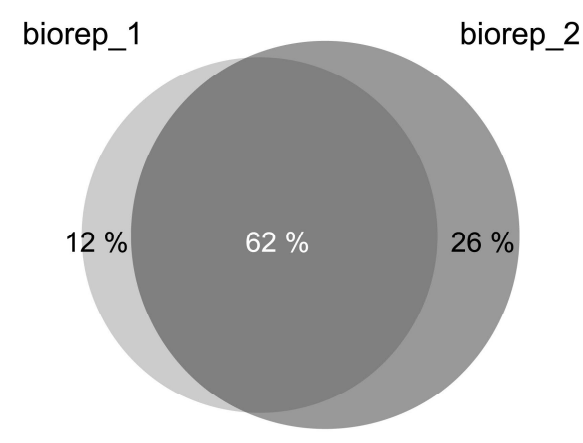

Supplementary Figure 1: Overlaps of unique residue-to-residue cross-links within biological replicates of cross-linked mitochondria derived from yeast grown on glycerol- or glucose containing medium. Unique residue-to-residue cross-links from the unfiltered and filtered data sets were compared between biological replicates of the glycerol (upper panel) and glucose condition (lower panel). Adapted from Linden et al. ${ }^{247}$ 


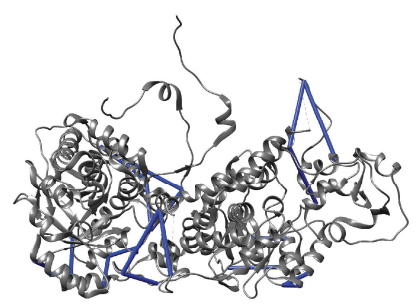

cytochrome b2 (Cyb2) intermembrane space (PDB: 1KBI)

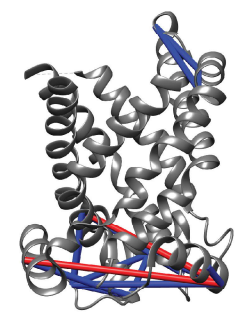

ADP,ATP carrier protein 2 (Pet9) inner membrane (PDB: 4C9G)

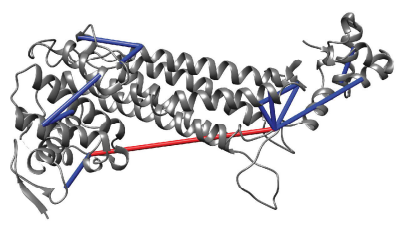

fumarate hydratase (Fum1) matrix (PDB: 1YFM)

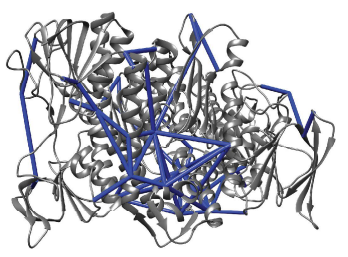

dihydrolipoyl dehydrogenase (Lpd1) matrix (PDB: 1V59)

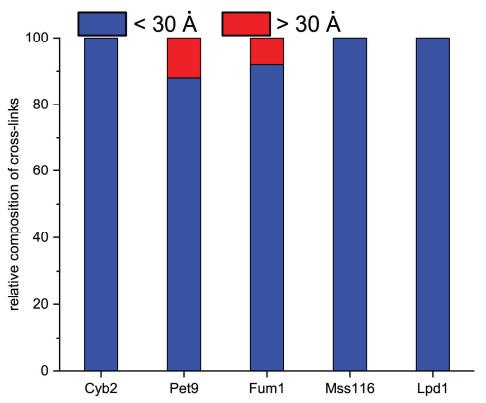

Supplementary Figure 2: Structural validation of selected cross-links identified in mitochondria derived from yeast grown on glycerol-containing medium. Identified cross-links were mapped onto available 3D structures of proteins located in different mitochondrial suborganellar compartments. Bar diagram shows the relative composition for each displayed structure. Blue bars, satisfying distance constraint $(<30 \AA)$; red bars, violated distance constraint $(>30 \AA)$. Structures were illustrated by UCSF Chimera ${ }^{274}$ in combination with Xlink Analyzer ${ }^{275}$. 


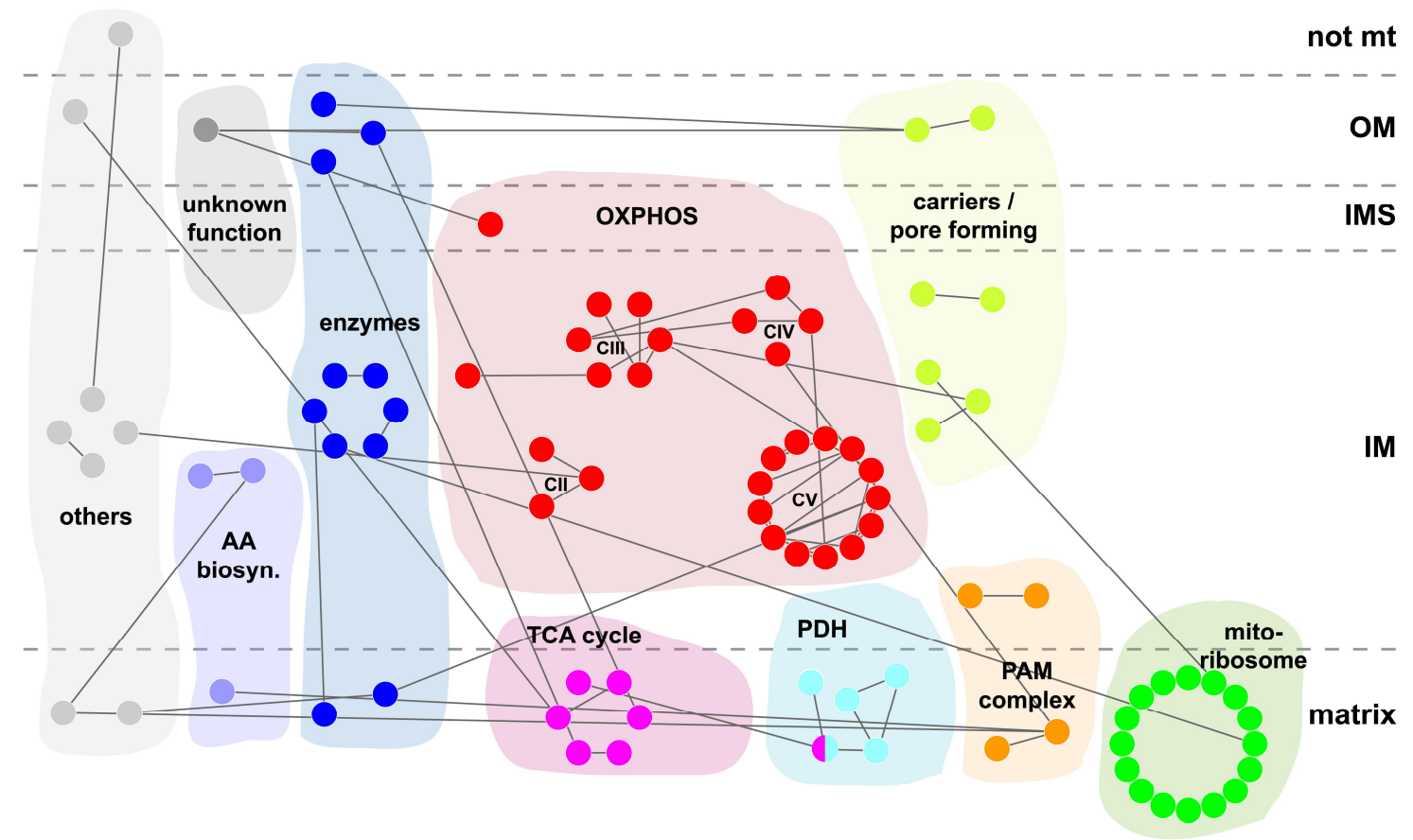

Supplementary Figure 3: Global interprotein cross-linking network identified in both mitochondria derived from yeast grown on glycerol- and glucose-containing medium. Localizations based on Vögtle et al. ${ }^{142}$ Thickness of the edges is proportional to the number of unique residue-to-residue cross-links. OM, outer membrane; IMS, intermembrane space; IM, inner membrane; ambig, ambiguous; not $\mathrm{mt}$, not mitochondrial; AA biosyn., amino acid biosynthesis; OXPHOS, oxidative phosphorylation system; TCA, tricarboxylic acid; PDH, pyruvate dehydrogenase complex; PAM, presequence translocase-associated motor, mitoribosome, mitochondrial ribosome. Networks visualized by Cytoscape ${ }^{269}$. Adapted from Linden et al. ${ }^{247}$ 

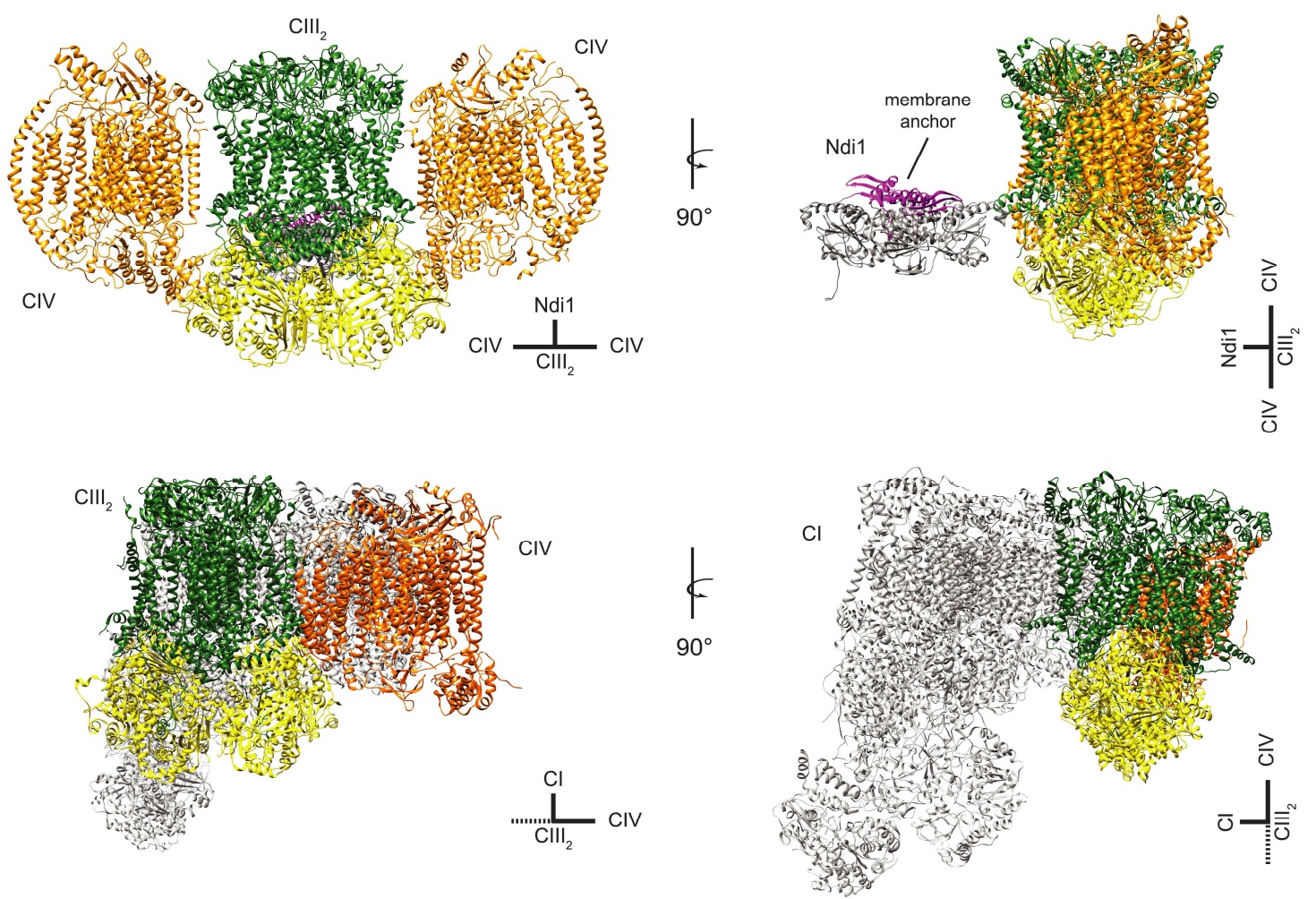

Supplementary Figure 4: Comparison of yeast and mammalian ETC supercomplex structures. Putative Ndi1CIII $\mathrm{CIV}_{2}$ supercomplex structure of yeast (upper panel) and the mammalian $\mathrm{CICIII}_{2} \mathrm{CIV}$ supercomplex (lower panel, Sus scrofa). Schematic T-pieces illustrate the orientation of all three complexes. Green, complex III; orange, complex IV; gray, Ndi1 and complex I; light purple, membrane anchor of Ndi1; yellow, subunits Cor1 and Qcr2 of CIII; IMS, intermembrane space; IM, inner membrane. PDB $\mathrm{Clll}_{2} \mathrm{CIV}_{2}$ supercomplex: 6HU991; PDB Ndi1: 4G73263; PDB $\mathrm{CICll}_{2} \mathrm{CIV}$ supercomplex: 5GUP92. Adapted from Linden et al.247 


\begin{tabular}{|c|c|c|c|c|c|}
\hline & o & ¿ & $0^{0}$ & 5 & 5 \\
\hline \multicolumn{6}{|c|}{ interprotein cross-links } \\
\hline \multirow[t]{2}{*}{ Min8 } & YPR010C-A & $*, * * *$ & Cox12 & 2 & 7 \\
\hline & & $* \star *$ & Pet9 & 1 & 4 \\
\hline Nat2 & YGR147C & & Mdh1 & 1 & 2 \\
\hline Rcf3 & YBR255C-A & ** & Cox 12 & 1 & 5 \\
\hline \multirow[t]{2}{*}{ Ycp4 } & YCR004C & & Hsp60 & 1 & 6 \\
\hline & & $* * *$ & Pst2 & 1 & 23 \\
\hline Aim17 & YHL021C & & Cpr3 & 1 & 5 \\
\hline Coq21 & YBR230W-A & * & Coq5 & 1 & 3 \\
\hline Dpi8 & YJL133C-A & $*, * \star *$ & Kgd1 & 1 & 34 \\
\hline \multirow[t]{2}{*}{ Fmp16 } & YDR070C & *** & Aco1 & 1 & 2 \\
\hline & YDR061W & & IIv5 & 2 & 10 \\
\hline \multicolumn{6}{|c|}{ intraprotein cross-links } \\
\hline Fmp10 & YER182W & $* * *$ & & 1 & 6 \\
\hline Fmp41 & YNL168C & $* * *$ & & 1 & 13 \\
\hline \multirow[t]{4}{*}{ Mco10 } & YOR020W-A & $* * *$ & & 1 & 4 \\
\hline & YDR061W & $* * *$ & & 1 & 5 \\
\hline & YGR266W & $* * *$ & & 1 & 2 \\
\hline & YKR070W & $* * *$ & & 1 & 2 \\
\hline Fmp10 & YER182W & $* * *$ & & 2 & 11 \\
\hline Fmp41 & YNL168C & $* * *$ & & 2 & 26 \\
\hline Ego4 & YNR034W-A & & & 1 & 7 \\
\hline Mco10 & YOR020W-A & $* * *$ & & 1 & 3 \\
\hline \multirow[t]{2}{*}{ Mcy1 } & YGR012W & $* * *$ & & 2 & 13 \\
\hline & YKR070W & *** & & 1 & 24 \\
\hline
\end{tabular}

Supplementary Figure 5: Uncharacterized proteins identified in mitochondria derived from yeast grown on glycerol- or glucose-containing medium. List of uncharacterized proteins that showed interprotein cross-links to other proteins (top part of the table) or intraprotein cross-links (bottom part of the table). Red, identified in the glycerol data set; blue, identified in the glucose data set. *, described in Morgenstern et al..$^{72}$; ${ }^{* *}$, described in Römpler et al. ${ }^{119}$; ***, identified in Makepeace et al. ${ }^{102}$; \#unique, number of unique residue-to-residue cross-links; \#CSMs, number of cross-linked peptides spectrum matches. Adapted from Linden et al. ${ }^{247}$ 


\subsection{Supplementary tables}

Supplementary Table 1: pLink 1 search results of the quantitative XL-MS approach for the comparison of residue-to-residue cross-links identified in mitochondria derived from yeast grown on glycerol- or glucose-containing medium. File provided on a CD-ROM attached to the hardcopy version of this thesis.

Supplementary Table 2: pLink 1 search results of the qualitative XL-MS approaches. Residue-toresidue cross-links identified in mitochondria derived from yeast grown on glycerol- or glucosecontaining medium and from the $r \mathrm{O}^{\circ}$ yeast strain. File provided on a CD-ROM attached to the hardcopy version of this thesis.

Supplementary Table 3: pLink 1 search results of the qualitative XL-MS approach with protein SEC as prefractionation. Residue-to-residue cross-links identified in mitochondria derived from yeast grown on glycerol-containing medium. File provided on a CD-ROM attached to the hardcopy version of this thesis.

Supplementary Table 4: Protein identifications and peptides per fraction after immunoprecipitation of Min8 ${ }^{F L A G}$ in combination with oxidative cross-linking. File provided on a CD-ROM attached to the hardcopy version of this thesis.

Supplementary Table 5: pLink 2 search results of the XL-MS approach of human mitoplasts. Residue-to-residue cross-links identified in mitoplasts generated from human mitochondria derived from HEK293T cells. File provided on a CD-ROM attached to the hardcopy version of this thesis.

Supplementary Table 6: Protein lists for generating databases including the 400 most abundant proteins. Databases were used for pLink 1 searches after cross-linking yeast mitochondria. File provided on a CD-ROM attached to the hardcopy version of this thesis.

Supplementary Table 7: Summary of so far known proteins that are part of the translocases of outer and inner membrane (TOM/TIM) complexes in $S$. cerevisiae ${ }^{21}$. ' $X$ ' indicates that the specific protein was identified as cross-linked in the respective data set $r h 0^{\circ}$, glucose or glycerol.

\begin{tabular}{|c|c|c|}
\hline \multirow[b]{2}{*}{ proteins } & \multicolumn{2}{|r|}{ cross-linked in } \\
\hline & $r h o^{\circ}$ & glucose glycerol \\
\hline \multicolumn{3}{|c|}{ TOM complex } \\
\hline $\begin{array}{l}\text { Tom5 } \\
\text { Tom6 } \\
\text { Tom7 }\end{array}$ & $X$ & \\
\hline
\end{tabular}




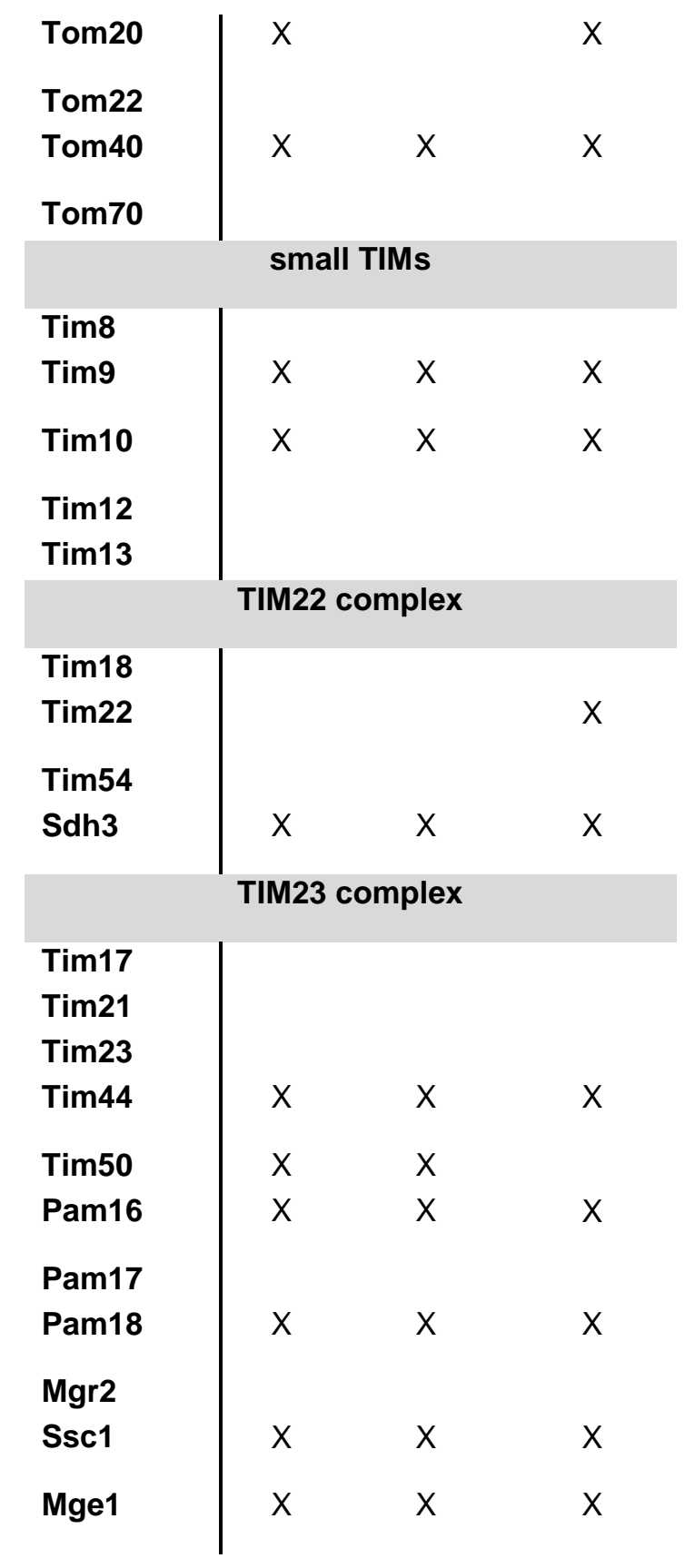


Supplementary Table 8: Raw files acquired and used for cross-linked peptide database search after cross-linking mitochondria derived from yeast grown on glycerol-containing medium.

\begin{tabular}{|c|c|c|}
\hline $\begin{array}{l}\text { C_Lee_011216_ymitos } \\
\text { WT_Gly_BS3_XL_10_ } \\
\text { R1.raw }\end{array}$ & $\begin{array}{l}\text { C_Lee_011216_ymitos_WT } \\
\text { _Gly_BS3_XL_16_R1.raw }\end{array}$ & $\begin{array}{l}\text { I_Parfentev_A_Linden_2606 } \\
\text { 17_180717_ymitos_WTGly_ } \\
\text { A10b.raw }\end{array}$ \\
\hline $\begin{array}{l}\text { C_Lee_011216_ymitos } \\
\text { WT_Gly_BS3_XL_10_ } \\
\text { R2.raw }\end{array}$ & $\begin{array}{l}\text { C_Lee_011216_ymitos_WT } \\
\text { _Gly_BS3_XL_16_R2.raw }\end{array}$ & $\begin{array}{l}\text { I_Parfentev_A_Linden_2606 } \\
\text { 17_180717_ymitos_WTGly_ } \\
\text { A11.raw }\end{array}$ \\
\hline $\begin{array}{l}\text { C_Lee_011216_ymitos } \\
\text { WT_Gly_BS3_XL_11_ } \\
\text { R1.raw }\end{array}$ & $\begin{array}{l}\text { C_Lee_011216_ymitos_WT } \\
\text { _Gly_BS3_XL_17_R1.raw }\end{array}$ & $\begin{array}{l}\text { I_Parfentev_A_Linden_2606 } \\
\text { 17_180717_ymitos_WTGly_ } \\
\text { A11b.raw }\end{array}$ \\
\hline $\begin{array}{l}\text { C_Lee_011216_ymitos } \\
\text { WT_Gly_BS3_XL_11_ } \\
\text { R2.raw }\end{array}$ & $\begin{array}{l}\text { C_Lee_011216_ymitos_WT } \\
\text { _Gly_BS3_XL_17_R2.raw }\end{array}$ & $\begin{array}{l}\text { I_Parfentev_A_Linden_2606 } \\
17 \text { 180717_ymitos_WTGly_ } \\
\text { A12.raw }\end{array}$ \\
\hline $\begin{array}{l}\text { C_Lee_011216_ymitos } \\
\text { WT_Gly_BS3_XL_12_ } \\
\text { R1.raw }\end{array}$ & $\begin{array}{l}\text { C_Lee_011216_ymitos_WT } \\
\text { _Gly_BS3_XL_18_R1.raw }\end{array}$ & $\begin{array}{l}\text { I_Parfentev_A_Linden_2606 } \\
17 \text { 180717_ymitos_WTGly_ } \\
\text { A12b.raw }\end{array}$ \\
\hline $\begin{array}{l}\text { C_Lee_011216_ymitos } \\
\text { WT_Gly_BS3_XL_12_ } \\
\text { R2.raw }\end{array}$ & $\begin{array}{l}\text { C_Lee_011216_ymitos_WT } \\
\text { _Gly_BS3_XL_18_R2.raw }\end{array}$ & $\begin{array}{l}\text { I_Parfentev_A_Linden_2606 } \\
\text { 17_180717_ymitos_WTGly_ } \\
\text { A13.raw }\end{array}$ \\
\hline $\begin{array}{l}\text { C_Lee_011216_ymitos } \\
\text { WT_Gly_BS3_XL_13_ } \\
\text { R1.raw }\end{array}$ & $\begin{array}{l}\text { C_Lee_011216_ymitos_WT } \\
\text { _Gly_BS3_XL_19to21_R1.r } \\
\text { aw }\end{array}$ & $\begin{array}{l}\text { I_Parfentev_A_Linden_2606 } \\
\text { 17_180717_ymitos_WTGly_ } \\
\text { A13b.raw }\end{array}$ \\
\hline $\begin{array}{l}\text { C_Lee_011216_ymitos } \\
\text { WT_Gly_BS3_XL_13 } \\
\text { R2a.raw }\end{array}$ & $\begin{array}{l}\text { C_Lee_011216_ymitos_WT } \\
\text { _Gly_BS3_XL_19to21_R2.r } \\
\text { aw }\end{array}$ & $\begin{array}{l}\text { I_Parfentev_A_Linden_2606 } \\
17 \_180717 \text { ymitos_WTGly_ } \\
\text { A14.raw }\end{array}$ \\
\hline $\begin{array}{l}\text { C_Lee_011216_ymitos } \\
\text { WT_Gly_BS3_XL_14_ } \\
\text { R1a.raw }\end{array}$ & $\begin{array}{l}\text { C_Lee_011216_ymitos_WT } \\
\text { _Gly_BS3_XL_7to9_R1.raw }\end{array}$ & $\begin{array}{l}\text { I_Parfentev_A_Linden_2606 } \\
\text { 17_180717_ymitos_WTGly_ } \\
\text { A14b.raw }\end{array}$ \\
\hline $\begin{array}{l}\text { C_Lee_011216_ymitos } \\
\text { WT_Gly_BS3_XL_14_ } \\
\text { R2a.raw }\end{array}$ & $\begin{array}{l}\text { C_Lee_011216_ymitos_WT } \\
\text { _Gly_BS3_XL_7to9_R2.raw }\end{array}$ & $\begin{array}{l}\text { I_Parfentev_A_Linden_2606 } \\
\text { 17_180717_ymitos_WTGly } \\
\text { A9.raw }\end{array}$ \\
\hline $\begin{array}{l}\text { C_Lee_011216_ymitos } \\
\text { WT_Gly_BS3_XL_15_ } \\
\bar{R} 1 \text {.raw }\end{array}$ & $\begin{array}{l}\text { I_Parfentev_A_Linden_2606 } \\
\text { 17_180717_ymitos_WTGly_ } \\
\text { A10.raw }\end{array}$ & $\begin{array}{l}\text { I_Parfentev_A_Linden_2606 } \\
17 \text { 180717_ymitos_WTGly } \\
\text { A9b.raw }\end{array}$ \\
\hline C Lee_011 & IT Glv BS3 XL 15 R2 ra & \\
\hline
\end{tabular}

Supplementary Table 9: Raw files acquired and used for cross-linked peptide database search after cross-linking mitochondria derived from yeast grown on glucose-containing medium.

\begin{tabular}{|l|l|l|}
\hline $\begin{array}{l}\text { C_Lee_261116_ymitos_W } \\
\text { T_Glu_BS3_XL_10_R1.ra } \\
\text { w }\end{array}$ & $\begin{array}{l}\text { C_Lee_261116_ymitos_WT } \\
\text { _Glu_BS3_XL_17_R1.raw }\end{array}$ & $\begin{array}{l}\text { C_Lee_300117_ymitos } \\
\text { Glu_BS3_XL_13_R1.raw }\end{array}$ \\
\hline $\begin{array}{l}\text { C_Lee_261116_ymitos_W } \\
\text { T_Glu_BS3_XL_10_R2.ra } \\
\text { w }\end{array}$ & $\begin{array}{l}\text { C_Lee_261116_ymitos_WT } \\
\text { _Glu_BS3_XL_17_R2.raw }\end{array}$ & $\begin{array}{l}\text { C_Lee_300117_ymitos_ } \\
\text { Glu_BS3_XL_13_R2.raw }\end{array}$ \\
\hline $\begin{array}{l}\text { C_Lee_261116_ymitos_W } \\
\text { T_Glu_BS3_XL_11_R1.ra } \\
\text { w }\end{array}$ & $\begin{array}{l}\text { C_Lee_261116_ymitos_WT } \\
\text {-Glu_BS3_XL_18_R1.raw }\end{array}$ & $\begin{array}{l}\text { C_Lee_300117_ymitos_ } \\
\text { Glu_BS3_XL_14_R1.raw }\end{array}$ \\
\hline $\begin{array}{l}\text { C_Lee_261116_ymitos_W } \\
\text { T_Glu_BS3_XL_11_R2.ra } \\
\text { w }\end{array}$ & $\begin{array}{l}\text { C_Lee_261116_ymitos_WT } \\
\text {-Glu_BS3_XL_18_R2.raw }\end{array}$ & $\begin{array}{l}\text { C_Lee_300117_ymitos_ } \\
\text { Glu_BS3_XL_14_R2.raw }\end{array}$ \\
\hline
\end{tabular}




\begin{tabular}{|c|c|c|}
\hline $\begin{array}{l}\text { C_Lee_261116_ymitos_W } \\
\text { T_Glu_BS3_XL_12_R1.ra } \\
\text { w }\end{array}$ & $\begin{array}{l}\text { C_Lee_261116_ymitos_WT } \\
\text { _Glu_BS3_XL_19to21_R1.r } \\
\text { aw }\end{array}$ & $\begin{array}{l}\text { C_Lee_300117_ymitos } \\
\text { Glu_BS3_XL_15_R1.raw }\end{array}$ \\
\hline $\begin{array}{l}\text { C_Lee_261116_ymitos_W } \\
\text { T_Glu_BS3_XL_12_R2.ra } \\
\text { w }\end{array}$ & $\begin{array}{l}\text { C_Lee_261116_ymitos_WT } \\
\text { _Glu_BS3_XL_19to21_R2.r } \\
\text { aw }\end{array}$ & $\begin{array}{l}\text { C_Lee_300117_ymitos } \\
\text { Glu_BS3_XL_15_R2.raw }\end{array}$ \\
\hline $\begin{array}{l}\text { C_Lee_261116_ymitos_W } \\
\text { T_Glu_BS3_XL_13_R1a.ra } \\
\text { w }\end{array}$ & $\begin{array}{l}\text { C_Lee_261116_ymitos_WT } \\
\text { _Glu_BS3_XL_7to9_R1.raw }\end{array}$ & $\begin{array}{l}\text { C_Lee_300117_ymitos } \\
\text { Glu_BS3_XL_16_R1.raw }\end{array}$ \\
\hline $\begin{array}{l}\text { C_Lee_261116_ymitos_W } \\
\text { T_Glu_BS3_XL_13_R2a.ra } \\
\text { w }\end{array}$ & $\begin{array}{l}\text { C_Lee_261116_ymitos_WT } \\
\text { _Glu_BS3_XL_7to9_R2.raw }\end{array}$ & $\begin{array}{l}\text { C_Lee_300117_ymitos } \\
\text { Glu_BS3_XL_16_R2.raw }\end{array}$ \\
\hline $\begin{array}{l}\text { C_Lee_261116_ymitos_W } \\
\text { T_Glu_BS3_XL_14_R1.ra } \\
\text { w }\end{array}$ & $\begin{array}{l}\text { C_Lee_300117_ymitos_Glu } \\
\text { _BS3_XL_10_R1.raw }\end{array}$ & $\begin{array}{l}\text { C_Lee_300117_ymitos } \\
\text { Glu_BS3_XL_17_R1.raw }\end{array}$ \\
\hline $\begin{array}{l}\text { C_Lee_261116_ymitos_W } \\
\text { T_Glu_BS3_XL_14_R2.ra } \\
\text { w }\end{array}$ & $\begin{array}{l}\text { C_Lee_300117_ymitos_Glu } \\
\text { _BS3_XL_10_R2.raw }\end{array}$ & $\begin{array}{l}\text { C_Lee_300117_ymitos } \\
\text { Glu_BS3_XL_17_R2.raw }\end{array}$ \\
\hline $\begin{array}{l}\text { C_Lee_261116_ymitos_W } \\
\text { T_Glu_BS3_XL_15_R1.ra } \\
\text { w }\end{array}$ & $\begin{array}{l}\text { C_Lee_300117_ymitos_Glu } \\
\text { _BS3_XL_11_R1.raw }\end{array}$ & $\begin{array}{l}\text { C_Lee_300117_ymitos } \\
\text { Glu_BS3_XL_18_R1.raw }\end{array}$ \\
\hline $\begin{array}{l}\text { C_Lee_261116_ymitos_W } \\
\text { T_Glu_BS3_XL_15_R2.ra } \\
\text { w }\end{array}$ & $\begin{array}{l}\text { C_Lee_300117_ymitos_Glu } \\
\text { _BS3_XL_11_R2.raw }\end{array}$ & $\begin{array}{l}\text { C_Lee_300117_ymitos } \\
\text { Glu_BS3_XL_18_R2.raw }\end{array}$ \\
\hline $\begin{array}{l}\text { C_Lee_261116_ymitos_W } \\
\text { T_Glu_BS3_XL_16_R1a.ra } \\
\text { w }\end{array}$ & $\begin{array}{l}\text { C_Lee_300117_ymitos_Glu } \\
\text { _BS3_XL_12_R1.raw }\end{array}$ & $\begin{array}{l}\text { C_Lee_300117_ymitos } \\
\text { Glu_BS3_XL_8to9_R1.ra } \\
\text { w }\end{array}$ \\
\hline $\begin{array}{l}\text { C_Lee_261116_ymitos_W } \\
\text { T_Glu_BS3_XL_16_R2.ra } \\
\text { w }\end{array}$ & $\begin{array}{l}\text { C_Lee_300117_ymitos_Glu } \\
\text { _BS3_XL_12_R2.raw }\end{array}$ & $\begin{array}{l}\text { C_Lee_300117_ymitos } \\
\text { Glu_BS3_XL_8to9_R2.ra } \\
\text { w }\end{array}$ \\
\hline
\end{tabular}

Supplementary Table 10: Raw files acquired and used for cross-linked peptide database search after cross-linking mitochondria derived from the $r \mathrm{o}^{\circ}$ yeast strain.

\begin{tabular}{|c|c|c|}
\hline $\begin{array}{l}\text { C_Lee_241116_ymitos_r } \\
\text { ho0_BS3_XL_10_R1.raw }\end{array}$ & $\begin{array}{l}\text { C_Lee_241116_ymitos_rho } \\
\text { 0_BS3_XL_17_R2.raw }\end{array}$ & $\begin{array}{l}\text { C_Lee_270117_ymitos_rho } \\
0 \text { BS3_XL_14_R1.raw }\end{array}$ \\
\hline $\begin{array}{l}\text { C_Lee_241116_ymitos_r } \\
\text { ho0_BS3 XL 10 R2.raw }\end{array}$ & $\begin{array}{l}\text { C_Lee_241116_ymitos_rho } \\
0 \text { BS3 XL_18_R1.raw }\end{array}$ & $\begin{array}{l}\text { C_Lee_270117_ymitos_rho } \\
0 \text { BS3_XL_14_R2.raw }\end{array}$ \\
\hline $\begin{array}{l}\text { C_Lee_241116_ymitos_r } \\
\text { ho0_BS3_XL_11_R1.raw }\end{array}$ & $\begin{array}{l}\text { C_Lee_241116_ymitos_rho } \\
\text { 0_BS3_XL_18_R2a.raw }\end{array}$ & $\begin{array}{l}\text { C_Lee_270117_ymitos_rho } \\
0 \text { BS3_XL_15_R1.raw }\end{array}$ \\
\hline $\begin{array}{l}\text { C_Lee_241116_ymitos_r } \\
\text { ho0_BS3_XL_11_R2.raw }\end{array}$ & $\begin{array}{l}\text { C_Lee_241116_ymitos_rho } \\
\text { 0_BS3_XL_19to21_R1a.ra } \\
\text { w }\end{array}$ & $\begin{array}{l}\text { C_Lee_270117_ymitos_rho } \\
\text { 0_BS3_XL_15_R2.raw }\end{array}$ \\
\hline $\begin{array}{l}\text { C_Lee_241116_ymitos_r } \\
\text { ho0_BS3 XL_12_R1.raw }\end{array}$ & $\begin{array}{l}\text { C_Lee_241116_ymitos_rho } \\
\text { 0_BS3_XL_19to21_R2.raw }\end{array}$ & $\begin{array}{l}\text { C_Lee_270117_ymitos_rho } \\
0 \text { BS3_XL_16_R1.raw }\end{array}$ \\
\hline $\begin{array}{l}\text { C_Lee_241116_ymitos_r } \\
\text { ho0_BS3 XL_12_R2.raw }\end{array}$ & $\begin{array}{l}\text { C_Lee_241116_ymitos_rho } \\
\text { 0_BS3_XL 7to9_R1.raw }\end{array}$ & $\begin{array}{l}\text { C_Lee_270117_ymitos_rho } \\
0 \text { BS3_XL 16_R2.raw }\end{array}$ \\
\hline $\begin{array}{l}\text { C_Lee_241116_ymitos_r } \\
\text { ho0_BS3_XL_13_R1.raw }\end{array}$ & $\begin{array}{l}\text { C_Lee_241116_ymitos_rho } \\
\text { 0_BS3_XL_7to9_R2.raw }\end{array}$ & $\begin{array}{l}\text { C_Lee_270117_ymitos_rho } \\
0 \text { _BS3_XL_17_R1.raw }\end{array}$ \\
\hline $\begin{array}{l}\text { C_Lee } 241116 \text { ymitos_r } \\
\text { ho0_BS3_XL_13_R2.raw }\end{array}$ & $\begin{array}{l}\text { C_Lee_270117_ymitos_rho } \\
0 \text { BS3_XL_10_R1.raw }\end{array}$ & $\begin{array}{l}\text { C_Lee_270117_ymitos_rho } \\
0 \text { BS3_XL 17 R2.raw }\end{array}$ \\
\hline
\end{tabular}




\begin{tabular}{|c|c|c|}
\hline $\begin{array}{l}\text { C_Lee_241116_ymitos_r } \\
\text { ho0_BS3_XL_14_R1.raw }\end{array}$ & $\begin{array}{l}\text { C_Lee_270117_ymitos_rho } \\
\text { 0_BS3_XL_10_R2.raw }\end{array}$ & $\begin{array}{l}\text { C_Lee_270117_ymitos_rho } \\
\text { 0_BS3_XL_18_R1.raw }\end{array}$ \\
\hline $\begin{array}{l}\text { C_Lee_241116_ymitos_r } \\
\text { ho0_BS3 XL_14_R2.raw }\end{array}$ & $\begin{array}{l}\text { C_Lee_270117_ymitos_rho } \\
0 \text { BS3_XL 11_R1.raw }\end{array}$ & $\begin{array}{l}\text { C_Lee_270117_ymitos_rho } \\
0 \text { BS3 XL 18_R2.raw }\end{array}$ \\
\hline $\begin{array}{l}\text { C_Lee_241116_ymitos_r } \\
\text { ho0_BS3_XL_15_R1.raw }\end{array}$ & $\begin{array}{l}\text { C_Lee_270117_ymitos_rho } \\
\text { 0_BS3_XL_11_R2.raw }\end{array}$ & $\begin{array}{l}\text { C_Lee_270117_ymitos_rho } \\
0 \_ \text {BS3_XL_19to21_R1_raw }\end{array}$ \\
\hline $\begin{array}{l}\text { C_Lee_241116_ymitos_r } \\
\text { ho0_BS3 XL_15_R2.raw }\end{array}$ & $\begin{array}{l}\text { C_Lee_270117_ymitos_rho } \\
0 \text { BS3_XL_12_R1.raw }\end{array}$ & $\begin{array}{l}\text { C_Lee_270117_ymitos_rho } \\
0 \text { BSS3XL_19to21_R2.raw }\end{array}$ \\
\hline $\begin{array}{l}\text { C_Lee_241116_ymitos_r } \\
\text { ho0_BS3_XL_16_R1.raw }\end{array}$ & $\begin{array}{l}\text { C_Lee_270117_ymitos_rho } \\
0 \_ \text {BS3_XL_12_R2.raw }\end{array}$ & $\begin{array}{l}\text { C_Lee_270117_ymitos_rho } \\
\text { 0_BS3_XL_8to9_R1.raw }\end{array}$ \\
\hline $\begin{array}{l}\text { C_Lee_241116_ymitos_r } \\
\text { ho0_BS3_XL_16_R2.raw }\end{array}$ & $\begin{array}{l}\text { C_Lee_270117_ymitos_rho } \\
\text { 0_BS3_XL_13_R1.raw }\end{array}$ & $\begin{array}{l}\text { C_Lee_270117_ymitos_rho } \\
\text { 0_BS3_XL_8to9_R2.raw }\end{array}$ \\
\hline $\begin{array}{l}\text { C_Lee_241116_ymitos_r } \\
\text { ho0 BS3 XL } 17 \text { R1.raw }\end{array}$ & \multicolumn{2}{|c|}{ C_Lee_270117_ymitos_rho0_BS3_XL_13_R2.raw } \\
\hline
\end{tabular}

Supplementary Table 11: Raw files acquired and used for cross-linked peptide database search after cross-linking mitochondria derived from yeast grown on glycerol-containing medium and prefractionation by protein size exclusion chromatography.

\begin{tabular}{|c|c|c|}
\hline $\begin{array}{l}\text { C_Lee_010916_ymitos_BS } \\
\text { 3_XL_A13_A15_10_Rep1. } \\
\text { raw }\end{array}$ & $\begin{array}{l}\text { C_Lee_010916_ymitos_BS } \\
\text { 3_XL_B3_B4_15_Rep2.ra } \\
\text { w }\end{array}$ & $\begin{array}{l}\text { C_Lee_090916_ymitos_B } \\
\text { S3_XL_B13_C1_11_Rep1 } \\
\text { raw }\end{array}$ \\
\hline $\begin{array}{l}\text { C_Lee_010916_ymitos_BS } \\
\text { 3_XL_A13_A15_10_Rep2. } \\
\text { raw }\end{array}$ & $\begin{array}{l}\text { C_Lee_010916_ymitos_BS } \\
\text { 3_XL_B3_B4_16to19_Rep } \\
\text { 1.raw }\end{array}$ & $\begin{array}{l}\text { C_Lee_090916_ymitos_B } \\
\text { S3_XL_B13_C1_11_Rep2 } \\
\text {.raw }\end{array}$ \\
\hline $\begin{array}{l}\text { C_Lee_010916_ymitos_BS } \\
\text { 3_XL_A13_A15_11_Rep1. } \\
\text { raw }\end{array}$ & $\begin{array}{l}\text { C_Lee_010916_ymitos_BS } \\
\text { 3_XL_B3_B4_16to19_Rep } \\
\text { 2.raw }\end{array}$ & $\begin{array}{l}\text { C_Lee_090916_ymitos_B } \\
\text { S3_XL_B13_C1_12_Rep1 } \\
\text {.raw }\end{array}$ \\
\hline $\begin{array}{l}\text { C_Lee_010916_ymitos_BS } \\
\text { 3_XL_A13_A15_11_Rep2. } \\
\text { raw }\end{array}$ & $\begin{array}{l}\text { C_Lee_010916_ymitos_BS } \\
\text { 3_XL_B3_B4_7to9_Rep1.r } \\
\text { aw }\end{array}$ & $\begin{array}{l}\text { C_Lee_090916_ymitos_B } \\
\text { S3_XL_B13_C1_12_Rep2 } \\
\text { raw }\end{array}$ \\
\hline $\begin{array}{l}\text { C_Lee_010916_ymitos_BS } \\
\text { 3_XL_A13_A15_12_Rep1. } \\
\text { raw }\end{array}$ & $\begin{array}{l}\text { C_Lee_010916_ymitos_BS } \\
\text { 3_XL_B3_B4_7to9_Rep2.r } \\
\text { aw }\end{array}$ & $\begin{array}{l}\text { C_Lee_090916_ymitos_B } \\
\text { S3_XL_B13_C1_13_Rep1 } \\
\text {.raw }\end{array}$ \\
\hline $\begin{array}{l}\text { C_Lee_010916_ymitos_BS } \\
\text { 3_XL_A13_A15_12_Rep2. } \\
\text { raw }\end{array}$ & $\begin{array}{l}\text { C_Lee_010916_ymitos_BS } \\
\text { 3_XL_B5_B6_10_Rep1.ra } \\
\text { w }\end{array}$ & $\begin{array}{l}\text { C_Lee_090916_ymitos_B } \\
\text { S3_XL_B13_C1_13_Rep2 } \\
\text {.raw }\end{array}$ \\
\hline $\begin{array}{l}\text { C_Lee_010916_ymitos_BS } \\
\text { 3_XL_A13_A15_13_Rep1. } \\
\text { raw }\end{array}$ & $\begin{array}{l}\text { C_Lee_010916_ymitos_BS } \\
\text { 3_XL_B5_B6_10_Rep2.ra } \\
\text { w }\end{array}$ & $\begin{array}{l}\text { C_Lee_090916_ymitos_B } \\
\text { S3_XL_B13_C1_14_Rep1 } \\
\text {.raw }\end{array}$ \\
\hline $\begin{array}{l}\text { C_Lee_010916_ymitos_BS } \\
\text { 3_XL_A13_A15_13_Rep2. } \\
\text { raw }\end{array}$ & $\begin{array}{l}\text { C_Lee_010916_ymitos_BS } \\
\text { 3_XL_B5_B6_11_Rep1.ra } \\
\text { w }\end{array}$ & $\begin{array}{l}\text { C_Lee_090916_ymitos_B } \\
\text { S3_XL_B13_C1_14_Rep2 } \\
\text {.raw }\end{array}$ \\
\hline $\begin{array}{l}\text { C_Lee_010916_ymitos_BS } \\
\text { 3_XL_A13_A15_14_Rep1. } \\
\text { raw }\end{array}$ & $\begin{array}{l}\text { C_Lee_010916_ymitos_BS } \\
\text { 3_XL_B5_B6_11_Rep2.ra } \\
\text { w }\end{array}$ & $\begin{array}{l}\text { C_Lee_090916_ymitos_B } \\
\text { S3_XL_B13_C11_15_Rep1 } \\
\text {.raw }\end{array}$ \\
\hline $\begin{array}{l}\text { C_Lee_010916_ymitos_BS } \\
\text { 3_XL_A13_A15_14_Rep2. } \\
\text { raw }\end{array}$ & $\begin{array}{l}\text { C_Lee_010916_ymitos_BS } \\
\text { 3_XL_B5_B6_12_Rep1.ra } \\
\text { w }\end{array}$ & $\begin{array}{l}\text { C_Lee_090916_ymitos_B } \\
\text { S3_XL_B13_C1_15_Rep2 } \\
\text {.raw }\end{array}$ \\
\hline $\begin{array}{l}\text { C_Lee_010916_ymitos_BS } \\
\text { 3_XL_A13_A15_15_Rep1. } \\
\text { raw }\end{array}$ & $\begin{array}{l}\text { C_Lee_010916_ymitos_BS } \\
\text { 3_XL_B5_B6_12_Rep2.ra } \\
\text { w }\end{array}$ & $\begin{array}{l}\text { C_Lee_090916_ymitos_B } \\
\text { S3_XL_B13_C1_16_Rep1 } \\
\text { raw }\end{array}$ \\
\hline
\end{tabular}




\begin{tabular}{|c|c|c|}
\hline $\begin{array}{l}\text { C_Lee_010916_ymitos_BS } \\
\text { 3_XL_A13_A15_15_Rep2. } \\
\text { raw }\end{array}$ & $\begin{array}{l}\text { C_Lee_010916_ymitos_BS } \\
\text { 3_XL_B5_B6_13_Rep1.ra } \\
\text { w }\end{array}$ & $\begin{array}{l}\text { C_Lee_090916_ymitos_B } \\
\text { S3_XL_B13_C1_16_Rep2 } \\
\text { raw }\end{array}$ \\
\hline $\begin{array}{l}\text { Lee_010916_ymitos_BS } \\
\text { XL_A13_A15_16_Rep1. }\end{array}$ & $\begin{array}{l}\text { C_Lee_010916_ymitos_BS } \\
\text { 3_XL_B5_B6_13_Rep2.ra } \\
\text { w }\end{array}$ & $\begin{array}{l}\text { C_Lee_090916_ymitos_B } \\
\text { S3_XL_B13_C1_17to19_ } \\
\text { Rep1.raw }\end{array}$ \\
\hline $\begin{array}{l}\text { C_Lee_010916_ymitos_BS } \\
\text { 3_XL_A13_A15_16_Rep2. } \\
\text { raw }\end{array}$ & $\begin{array}{l}\text { C_Lee_010916_ymitos_BS } \\
\text { 3_XL_B5_B6_14_Rep1.ra } \\
\text { w }\end{array}$ & $\begin{array}{l}\text { C_Lee_090916_ymitos_B } \\
\text { S3_XL_B13_C1_17to19_ } \\
\text { Rep2.raw }\end{array}$ \\
\hline $\begin{array}{l}\text { C_Lee_010916_ymitos_BS } \\
\text { 3_XL_A13_A15_17to19_R } \\
\text { ep1.raw }\end{array}$ & $\begin{array}{l}\text { C_Lee_010916_ymitos_BS } \\
\text { 3_XL_B5_B6_14_Rep2.ra } \\
\text { w }\end{array}$ & $\begin{array}{l}\text { C_Lee_090916_ymitos_B } \\
\text { S3_XL_B13_C1_7to9_Re } \\
\text { p1.raw }\end{array}$ \\
\hline $\begin{array}{l}\text { C_Lee_010916_ymitos_BS } \\
\text { 3_XL_A13_A15_17to19_R } \\
\text { ep2.raw }\end{array}$ & $\begin{array}{l}\text { C_Lee_010916_ymitos_BS } \\
\text { 3_XL_B5_B6_15_Rep1.ra } \\
\text { w }\end{array}$ & $\begin{array}{l}\text { C_Lee_090916_ymitos_B } \\
\text { S3_XL_B13_C1_7to9_Re } \\
\text { p2.raw }\end{array}$ \\
\hline $\begin{array}{l}\text { C_Lee_010 } \\
\text { 3_XL_A13_ } \\
\text { 1.raw }\end{array}$ & $\begin{array}{l}\text { C_Lee_010916_ymitos_BS } \\
\text { 3_XL_B5_B6_15_Rep2.ra } \\
\text { w }\end{array}$ & $\begin{array}{l}\text { C_Lee_090916_ymitos_B } \\
\text { S3_XL_B7_B8_11_Rep1.r } \\
\text { aw }\end{array}$ \\
\hline $\begin{array}{l}\text { C_Lee_010 } \\
\text { 3_XL_A } 13 \text {.raw } \\
\text { 2.raw }\end{array}$ & $\begin{array}{l}\text { C_Lee_010916_ymitos_BS } \\
\text { 3_XL_B5_B6_16to18_Rep } \\
\text { 1.raw }\end{array}$ & $\begin{array}{l}\text { C_Lee_090916_ymitos_B } \\
\text { S3_XL_B7_B8_11_Rep2.r } \\
\text { aw }\end{array}$ \\
\hline $\begin{array}{l}\text { C_Lee_010 } \\
\text { 3_XL_B1_E } \\
w\end{array}$ & $\begin{array}{l}\text { C_Lee_010916_y } \\
\text { 3_XL_B5_B6_16t } \\
\text { 2.raw }\end{array}$ & $\begin{array}{l}\text { C_Lee_090916_ymitos_B } \\
\text { S3_XL_B7_B8_12_Rep1.r } \\
\text { aw }\end{array}$ \\
\hline $\begin{array}{l}\text { C_Lee } 010 \\
\text { 3_XL_B1_B } \\
\text { w }\end{array}$ & $\begin{array}{l}\text { C_Lee_010916_y } \\
\text { 3_XL_B5_B6_6to } \\
\text { aw }\end{array}$ & $\begin{array}{l}\text { C_Lee_090916_ymitos_B } \\
\text { S3_XL_B7_B8_12_Rep2.r } \\
\text { aw }\end{array}$ \\
\hline $\begin{array}{l}\text { C_Lee } 010 \\
\text { 3_XL_B1_B } \\
\text { w }\end{array}$ & $\begin{array}{l}\text { C_Lee_010916_. } \\
\text { 3_XL_B5_B6_6tc } \\
\text { aw }\end{array}$ & $\begin{array}{l}\text { C_Lee_090916_ymitos_B } \\
\text { S3_XL_B7_B8_13_Rep1.r } \\
\text { aw }\end{array}$ \\
\hline $\begin{array}{l}\text { C_Lee_010 } \\
\text { 3_XL_B1_E } \\
\text { w }\end{array}$ & $\begin{array}{l}\text { C_Lee_010916_ymitos_BS } \\
\text { 3_XL_B5_B6_9_Rep1.raw }\end{array}$ & $\begin{array}{l}\text { C_Lee_09 } \\
\text { S3_XL_B7 } \\
\text { aw }\end{array}$ \\
\hline $\begin{array}{l}\text { C_Lee_01 } \\
3 \_X L \_B 1 \_ \\
\text {w }\end{array}$ & $\begin{array}{l}\text { C_Lee_010916_y } \\
\text { 3_XL_B5_B6_9_ }\end{array}$ & $\begin{array}{l}\text { C_Lee_09 } \\
\text { S3_XL_B7 } \\
\text { aw }\end{array}$ \\
\hline $\begin{array}{l}\text { C_Lee_010916 } \\
\text { 3_XL_B1_B2_12 } \\
\text { w }\end{array}$ & $\begin{array}{l}\text { C_Lee_070916 } \\
\text { 3_XL_B7_B8_10 } \\
\text { w }\end{array}$ & $\begin{array}{l}\text { C_Lee_090 } \\
\text { S3_XL_B7 } \\
\text { aw }\end{array}$ \\
\hline $\begin{array}{l}\text { C_Lee_010 } \\
\text { 3_XL_B1_B } \\
\text { w }\end{array}$ & $\begin{array}{l}\text { C_Lee_070916_y } \\
\text { 3_XL_B7_B8_10 } \\
\text { w }\end{array}$ & $\begin{array}{l}\text { C_Lee_090916_ymitos_B } \\
\text { S3_XL_B7_B8_15_Rep1.r } \\
\text { aw }\end{array}$ \\
\hline $\begin{array}{l}\text { C_Lee_010 } \\
\text { 3_XL_B1_E } \\
\text { w }\end{array}$ & $\begin{array}{l}\text { C_Lee_070916_. } \\
\text { 3_XL_B7_B8_6tc }\end{array}$ & $\begin{array}{l}\text { C_Lee_090 } \\
\text { S3_XL_B7 } \\
\text { aw }\end{array}$ \\
\hline $\begin{array}{l}\text { C_Lee_010 } \\
\text { 3_XL_B1_E } \\
w\end{array}$ & $\begin{array}{l}\text { C_Lee_070916_y } \\
\text { 3_XL_B7_B8_6to } \\
\text { aw }\end{array}$ & $\begin{array}{l}\text { C_Lee_090 } \\
\text { S3_XL_B7 } \\
\text { ep1.raw }\end{array}$ \\
\hline $\begin{array}{l}\text { C_Lee_010s } \\
\text { 3_XL_B1_B } \\
w\end{array}$ & $\begin{array}{l}\text { C_Lee_070916_ymitos_BS } \\
\text { 3_XL_B7_B8_9_Rep1.raw }\end{array}$ & $\begin{array}{l}\text { C_Lee_090916_ym } \\
\text { S3_XL_B7_B8_16t }\end{array}$ \\
\hline $\begin{array}{l}\text { C_Lee_010916_yl } \\
\text { 3_XL_B1_B2_15_ } \\
\text { w }\end{array}$ & $\begin{array}{l}\text { C_Lee_070916_ymitos_BS } \\
\text { 3_XL_B7_B8_9_Rep2.raw }\end{array}$ & $\begin{array}{l}\text { C_Lee_090916_ymitos_B } \\
\text { S3_XL_B9_B10_10_Rep1 } \\
\text {.raw }\end{array}$ \\
\hline
\end{tabular}




\begin{tabular}{|c|c|c|}
\hline $\begin{array}{l}\text { C_Lee_010916_ymitos_BS } \\
\text { 3_XL_B1_B2_15_Rep2.ra } \\
\text { w }\end{array}$ & $\begin{array}{l}\text { C_Lee_090916_ymitos_BS } \\
\text { 3_XL_B11_B12_10_Rep1. } \\
\text { raw }\end{array}$ & $\begin{array}{l}\text { C_Lee_090916_ymitos_B } \\
\text { S3_XL_B9_B10_10_Rep2 } \\
\text {.raw }\end{array}$ \\
\hline $\begin{array}{l}\text { C_Lee_010916_ymitos_BS } \\
\text { 3_XL_B1_B2_16_Rep1.ra } \\
\text { w }\end{array}$ & $\begin{array}{l}\text { C_Lee_090916_ymitos_BS } \\
\text { 3_XL_B11_B12_10_Rep2. } \\
\text { raw }\end{array}$ & $\begin{array}{l}\text { C_Lee_090916_ymitos_B } \\
\text { S3_XL_B9_B10_11_Rep1 } \\
\text {.raw }\end{array}$ \\
\hline $\begin{array}{l}\text { C_Lee_010916_ymitos_BS } \\
\text { 3_XL_B1_B2_16_Rep2.ra } \\
\text { w }\end{array}$ & $\begin{array}{l}\text { C_Lee_090916_ymitos_BS } \\
\text { 3_XL_B11_B12_11_Rep1. } \\
\text { raw }\end{array}$ & $\begin{array}{l}\text { C_Lee_090916_ymitos_B } \\
\text { S3_XL_B9_B10_11_Rep2 } \\
\text {.raw }\end{array}$ \\
\hline $\begin{array}{l}\text { C_Lee_010916_ymitos_BS } \\
\text { 3_XL_B1_B2_17to19_Rep } \\
\text { 1.raw }\end{array}$ & $\begin{array}{l}\text { C_Lee_090916_ymitos_BS } \\
\text { 3_XL_B11_B12_11_Rep2. } \\
\text { raw }\end{array}$ & $\begin{array}{l}\text { C_Lee_090916_ymitos_B } \\
\text { S3_XL_B9_B10_12_Rep1 } \\
\text { raw }\end{array}$ \\
\hline $\begin{array}{l}\text { C_Lee_010916_ymitos_BS } \\
\text { 3_XL_B1_B2_17to19_Rep } \\
\text { 2.raw }\end{array}$ & $\begin{array}{l}\text { C_Lee_090916_ymitos_BS } \\
\text { 3_XL_B11_B12_12_Rep1. } \\
\text { raw }\end{array}$ & $\begin{array}{l}\text { C_Lee_090916_ymitos_B } \\
\text { S3_XL_B9_B10_12_Rep2 } \\
\text { raw }\end{array}$ \\
\hline $\begin{array}{l}\text { C_Lee_010916_ymitos_BS } \\
\text { 3_XL_B1_B2_7to9_Rep1.r } \\
\text { aw }\end{array}$ & $\begin{array}{l}\text { C_Lee_090916_ymitos_BS } \\
\text { 3_XL_B11_B12_12_Rep2. } \\
\text { raw }\end{array}$ & $\begin{array}{l}\text { C_Lee_090916_ymitos_B } \\
\text { S3_XL_B9_B10_13_Rep1 } \\
\text {.raw }\end{array}$ \\
\hline $\begin{array}{l}\text { C_Lee_010916_ymitos_BS } \\
\text { 3_XL_B1_B2_7to9_Rep2.r } \\
\text { aw }\end{array}$ & $\begin{array}{l}\text { C_Lee_090916_ymitos_BS } \\
\text { 3_XL_B11_B12_13_Rep1. } \\
\text { raw }\end{array}$ & $\begin{array}{l}\text { C_Lee_090916_ymitos_B } \\
\text { S3_XL_B9_B10_13_Rep2 } \\
\text { raw }\end{array}$ \\
\hline $\begin{array}{l}\text { C_Lee_010916_ymitos_BS } \\
\text { 3_XL_B3_B4_10_Rep1.ra } \\
\text { w }\end{array}$ & $\begin{array}{l}\text { C_Lee_090916_ymitos_BS } \\
\text { 3_XL_B11_B12_13_Rep2. } \\
\text { raw }\end{array}$ & $\begin{array}{l}\text { C_Lee_090916_ymitos_B } \\
\text { S3_XL_B9_B10_14_Rep1 } \\
\text {.raw }\end{array}$ \\
\hline $\begin{array}{l}\text { C_Lee_010916_ymitos_BS } \\
\text { 3_XL_B3_B4_10_Rep2.ra } \\
\text { w }\end{array}$ & $\begin{array}{l}\text { C_Lee_090916_ymitos_BS } \\
\text { 3_XL_B11_B12_14_Rep1. } \\
\text { raw }\end{array}$ & $\begin{array}{l}\text { C_Lee_090916_ymitos_B } \\
\text { S3_XL_B9_B10_14_Rep2 } \\
\text {.raw }\end{array}$ \\
\hline $\begin{array}{l}\text { C_Lee_010916_ymitos_BS } \\
\text { 3_XL_B3_B4_11_Rep1.ra } \\
\text { w }\end{array}$ & $\begin{array}{l}\text { C_Lee_090916_ymitos_BS } \\
\text { 3_XL_B11_B12_14_Rep2. } \\
\text { raw }\end{array}$ & $\begin{array}{l}\text { C_Lee_090916_ymitos_B } \\
\text { S3_XL_B9_B10_15_Rep1 } \\
\text {.raw }\end{array}$ \\
\hline $\begin{array}{l}\text { C_Lee_010916_ymitos_BS } \\
\text { 3_XL_B3_B4_11_Rep2.ra } \\
\text { w }\end{array}$ & $\begin{array}{l}\text { C_Lee_090916_ymitos_BS } \\
\text { 3_XL_B11_B12_15to17_R } \\
\text { ep1.raw }\end{array}$ & $\begin{array}{l}\text { C_Lee_090916_ymitos_B } \\
\text { S3_XL_B9_B10_15_Rep2 } \\
\text {.raw }\end{array}$ \\
\hline $\begin{array}{l}\text { C_Lee_010916_ymitos_BS } \\
\text { 3_XL_B3_B4_12_Rep1.ra } \\
\text { w }\end{array}$ & $\begin{array}{l}\text { C_Lee_090916_ymitos_BS } \\
\text { 3_XL_B11_B12_15to17_R } \\
\text { ep2.raw }\end{array}$ & $\begin{array}{l}\text { C_Lee_090916_ymitos_B } \\
\text { S3_XL_B9_B10_16_Rep1 } \\
\text {.raw }\end{array}$ \\
\hline $\begin{array}{l}\text { C_Lee_010916_ymitos_BS } \\
\text { 3_XL_B3_B4_12_Rep2.ra } \\
\text { w }\end{array}$ & $\begin{array}{l}\text { C_Lee_090916_ymitos_BS } \\
\text { 3_XL_B11_B12_6to8_Rep } \\
\text { 1.raw }\end{array}$ & $\begin{array}{l}\text { C_Lee_090916_ymitos_B } \\
\text { S3_XL_B9_B10_16_Rep2 } \\
\text {.raw }\end{array}$ \\
\hline $\begin{array}{l}\text { C_Lee_010916_ymitos_BS } \\
\text { 3_XL_B3_B4_13_Rep1.ra } \\
\text { w }\end{array}$ & $\begin{array}{l}\text { C_Lee_090916_ymitos_BS } \\
\text { 3_XL_B11_B12_6to8_Rep } \\
\text { 2.raw }\end{array}$ & $\begin{array}{l}\text { C_Lee_090916_ymitos_B } \\
\text { S3_XL_B9_B10_17to19_ } \\
\text { Rep1.raw }\end{array}$ \\
\hline $\begin{array}{l}\text { C_Lee_010916_ymitos_BS } \\
\text { 3_XL_B3_B4_13_Rep2.ra } \\
\text { w }\end{array}$ & $\begin{array}{l}\text { C_Lee_090916_ymitos_BS } \\
\text { 3_XL_B11_B12_9_Rep1.r } \\
\text { aw }\end{array}$ & $\begin{array}{l}\text { C_Lee_090916_ymitos_B } \\
\text { S3_XL_B9_B10_17to19_ } \\
\text { Rep2.raw }\end{array}$ \\
\hline $\begin{array}{l}\text { C_Lee_010916_ymitos_BS } \\
\text { 3_XL_B3_B4_14_Rep1.ra } \\
\text { w }\end{array}$ & $\begin{array}{l}\text { C_Lee_090916_ymitos_BS } \\
\text { 3_XL_B11_B12_9_Rep2.r } \\
\text { aw }\end{array}$ & $\begin{array}{l}\text { C_Lee_090916_ymitos_B } \\
\text { S3_XL_B9_B10_7to9_Re } \\
\text { p1.raw }\end{array}$ \\
\hline $\begin{array}{l}\text { C_Lee_010916_ymitos_BS } \\
\text { 3_XL_B3_B4_14_Rep2.ra } \\
\text { w }\end{array}$ & $\begin{array}{l}\text { C_Lee_090916_ymitos_BS } \\
\text { 3_XL_B13_C1_10_Rep1.r } \\
\text { aw }\end{array}$ & $\begin{array}{l}\text { C_Lee_090916_ymitos_B } \\
\text { S3_XL_B9_B10_7to9_Re } \\
\text { p2.raw }\end{array}$ \\
\hline $\begin{array}{l}\text { C_Lee_010916_ymitos_BS } \\
\text { 3_XL_B3_B4_15_Rep1.ra } \\
\text { w }\end{array}$ & \multicolumn{2}{|c|}{$\begin{array}{l}\text { C_Lee_090916_ymitos_BS3_XL_B13_C1_10_Rep2.ra } \\
\text { w }\end{array}$} \\
\hline
\end{tabular}


Supplementary Table 12: Raw files acquired and used for cross-linked peptide database search after cross-linking crude mitochondrial extract derived from yeast grown on glycerol-containing medium with DSS and BS3-d4.

\begin{tabular}{|l|l|l|}
\hline $\begin{array}{l}\text { A_Linden_131118_211118 } \\
\text { _ymitos_crude_fresh_1to1 } \\
\text { 10.raw }\end{array}$ & $\begin{array}{l}\text { A_Linden_131118_21111 } \\
\text { 8_ymitos_crude_fresh_1 to } \\
\text { 1_12.raw }\end{array}$ & $\begin{array}{l}\text { A_Linden_131118_211118 } \\
\text { ymitos_crude_fresh_1to1_ } \\
\text { 14.raw }\end{array}$ \\
\hline $\begin{array}{l}\text { A_Linden_131118_211118 } \\
\text { _ymitos_crude_fresh_1to1 } \\
\text { 11.raw }\end{array}$ & $\begin{array}{l}\text { A_Linden_131118_21111 } \\
\text { 8_ymitos_crude_fresh_1to } \\
\text { 1_13.raw }\end{array}$ & $\begin{array}{l}\text { A_Linden_131118_211118 } \\
\text { ymitos_crude_fresh_1to1_ } \\
\text { 7to9.raw }\end{array}$ \\
\hline
\end{tabular}

Supplementary Table 13: Raw files acquired and used for cross-linked peptide database search for the quantitation experiments of yeast mitochondria.

\begin{tabular}{|c|c|c|}
\hline $\begin{array}{l}\text {-Linden_231018_24101 } \\
\text { ymitos_glyd0- } \\
\text { lud4_10.raw }\end{array}$ & A_Linden_231018_251018 & $\begin{array}{l}\text { ymitos_quant_n } \\
\text { lud0-Glyd4_10.ra }\end{array}$ \\
\hline $\begin{array}{l}\text { Linden_231018_24101 } \\
\text { ymitos_glyd0- } \\
\text { ud4_11.raw }\end{array}$ & $\begin{array}{l}\text { A_Linden_231018_251018 } \\
\text { ymitos_glud0- } \\
\text { lyd4_11.raw }\end{array}$ & $\begin{array}{l}\text { 18_271118 } \\
\text { new_3rd_G } \\
\text { aw }\end{array}$ \\
\hline $\begin{array}{l}\text { Linden_231018_24101 } \\
\text { _ymitos_glyd0- } \\
\text { ud4_6to8.raw }\end{array}$ & $\begin{array}{l}\text { H_Linden_231018_251018 } \\
\text { ymitos_glud0- } \\
\text { lyd4_12.raw }\end{array}$ & $\begin{array}{l}\text { 18_271118 } \\
\text { new_3rd_G } \\
\text { 3.raw }\end{array}$ \\
\hline $\begin{array}{l}\text { LLinden_231018_24101 } \\
\text { _ymitos_glyd0- } \\
\text { lud4_9.raw }\end{array}$ & $\begin{array}{l}\text { A_Linden_231018_251018 } \\
\text { _ymitos_glud0-glyd4_9.raw }\end{array}$ & $\frac{\mathrm{ymi}}{\mathrm{lud} 0}$ \\
\hline $\begin{array}{l}\text { en_231018_25101 } \\
\text { s_glyd0- } \\
\text { lob.raw }\end{array}$ & $\begin{array}{l}\text { _Linden_231018_251018 } \\
\text { ymitos_glud0- } \\
\text { ylyd4_10b.raw }\end{array}$ & 8_271 \\
\hline $\begin{array}{l}\text { s_231018_25101 } \\
\text { 1b.raw }\end{array}$ & $\begin{array}{l}\text { ymitinden_231018_251018 } \\
\text { ymitos_glud0- } \\
\text { lyd4_11b.raw }\end{array}$ & $\begin{array}{l}\text { A_Lin } \\
\text { ymit } \\
\text { lud0-C }\end{array}$ \\
\hline $\begin{array}{l}\text { Linden_231018_25101 } \\
\text { ymitos_glyd0- } \\
\text { lud4_12.raw }\end{array}$ & $\begin{array}{l}\text { A_Linden_231018_251018 } \\
\text { ymitos_glud0- } \\
\text { ylyd4_6to8.raw }\end{array}$ & $\begin{array}{l}\text { A_Linde } \\
\text { lumitos } \\
\text { lud0-Gl }\end{array}$ \\
\hline S_231018_25101 & $\begin{array}{l}\text { A_Linden_231018_251018 } \\
\text { ymitos_glud0- }\end{array}$ & $\begin{array}{l}\text { A_Lin } \\
\text { Iumit } \\
\text { lud0-( }\end{array}$ \\
\hline $\begin{array}{l}31018 \_25101 \\
\text { do- }\end{array}$ & den_231018_251018 & $\begin{array}{l}\text { A_Li } \\
\text { lumi }\end{array}$ \\
\hline $\begin{array}{l}\text { Iden_231018_25101 } \\
\text { itos_glyd0- }\end{array}$ & $\begin{array}{l}\text { nden_231018_251018 } \\
\text { tos_glud0- }\end{array}$ & $\begin{array}{l}8 \_271 \\
\text { ew_3 } \\
a w\end{array}$ \\
\hline $\begin{array}{l}\text { nden_231018_25101 } \\
\text { itos_glyd0- }\end{array}$ & $\begin{array}{l}\text { nden_231018_251018 } \\
\text { tos_glud0- } \\
\text { t_14.raw }\end{array}$ & 8_271 \\
\hline $\begin{array}{l}\text { A_Linden_231018_25101 } \\
\text { 8_ymitos_glyd0- }\end{array}$ & $\begin{array}{l}\text { A_Linden_231018_251018 } \\
\text { _ymitos_glud0- }\end{array}$ & $\begin{array}{l}\text { 18_271118 } \\
\text { new_3rd_G } \\
\text { raw }\end{array}$ \\
\hline $\begin{array}{l}\text { en_231018_25101 } \\
\text { os_glyd0- }\end{array}$ & $\begin{array}{l}\text { A_Linden_231018_251018 } \\
\text { ymitos_glud0- } \\
\text { glyd4_12b.raw }\end{array}$ & $18 \_271$ \\
\hline
\end{tabular}




\begin{tabular}{|c|c|c|}
\hline $\begin{array}{l}\text { A_Linden_231018_25101 } \\
\text { 8_ymitos_glyd0- } \\
\text { glud4_9b.raw }\end{array}$ & $\begin{array}{l}\text { A_Linden_231018_251018 } \\
\text { _ymitos_glud0- } \\
\text { glyd4_9b.raw }\end{array}$ & $\begin{array}{l}\text { A_Linden_261118_271118 } \\
\text { _ymitos_quant_new_3rd_G } \\
\text { lud0-Glyd4_9b.raw }\end{array}$ \\
\hline $\begin{array}{l}\text { A_Linden_261118_29111 } \\
\text { 8_ymitos_quant_new_4th } \\
\text { _Glyd0-Glud4_10.raw }\end{array}$ & $\begin{array}{l}\text { A_Linden_261118_291118 } \\
\text { ymitos_quant_new_4th_G } \\
\text { lyd0-Glud4_11b.raw }\end{array}$ & $\begin{array}{l}\text { A_Linden_261118_291118 } \\
\text { ymitos_quant_new_4th_G } \\
\text { lyd0-Glud4_14.raw }\end{array}$ \\
\hline $\begin{array}{l}\text { A_Linden_261118_29111 } \\
\text { 8_ymitos_quant_new_4th } \\
\text { _Glyd0-Glud4_11.raw }\end{array}$ & $\begin{array}{l}\text { A_Linden_261118_291118 } \\
\text { ymitos_quant_new_4th_G } \\
\text { lyd0-Glud4_6to8.raw }\end{array}$ & $\begin{array}{l}\text { A_Linden_261118_291118 } \\
\text { ymitos_quant_new_4th_G } \\
\text { lyd0-Glud4_14b.raw }\end{array}$ \\
\hline $\begin{array}{l}\text { A_Linden_261118_29111 } \\
\text { 8_ymitos_quant_new_4th } \\
\text { _Glyd0-Glud4_12.raw }\end{array}$ & $\begin{array}{l}\text { A_Linden_261118_291118 } \\
\text { ymitos_quant_new_4th_G } \\
\text { lyd0-Glud4_6to8b.raw }\end{array}$ & $\begin{array}{l}\text { A_Linden_261118_291118 } \\
\text { ymitos_quant_new_4th_G } \\
\text { lyd0-Glud4_12b.raw }\end{array}$ \\
\hline $\begin{array}{l}\text { A_Linden_261118_29111 } \\
\text { 8_ymitos_quant_new_4th } \\
\text { _Glyd0-Glud4_13.raw }\end{array}$ & $\begin{array}{l}\text { A_Linden_261118_291118 } \\
\text { _ymitos_quant_new_4th_G } \\
\text { lyd0-Glud4_9.raw }\end{array}$ & $\begin{array}{l}\text { A_Linden_261118_291118 } \\
\text { _ymitos_quant_new_4th_G } \\
\text { lyd0-Glud4_13b.raw }\end{array}$ \\
\hline $\begin{array}{l}\text { A_Linden_261118_29111 } \\
\text { 8_ymitos_quant_new_4th } \\
\text { _Glyd0-Glud4_10b.raw }\end{array}$ & \multicolumn{2}{|c|}{$\begin{array}{l}\text { A_Linden_261118_291118_ymitos_quant_new_4th_Gly } \\
\text { d0-Glud4_9b.raw }\end{array}$} \\
\hline
\end{tabular}

Supplementary Table 14: Raw files acquired and used for cross-linked peptide database search after cross-linking human mitoplasts with BS3 and EDC.

\begin{tabular}{|c|c|c|}
\hline $\begin{array}{l}\text { ALinden_121119_hMP } \\
\text { BS3 10.raw }\end{array}$ & $\begin{array}{l}\text { ALinden_141119_hMP_BS3 } \\
\text { gel 19.raw }\end{array}$ & $\begin{array}{l}\text { ALinden_281119_310120_h } \\
\text { MP EDC gel 21.raw }\end{array}$ \\
\hline $\begin{array}{l}\text { ALinden_121119_hMP } \\
\text { BS3 10b.raw }\end{array}$ & $\begin{array}{l}\text { ALinden_141119_hMP_BS3 } \\
\text { gel 2.raw }\end{array}$ & $\begin{array}{l}\text { ALinden_281119_310120_h } \\
\text { MP EDC gel 22.raw }\end{array}$ \\
\hline $\begin{array}{l}\text { ALinden_121119_hMP } \\
\text { BS3_11.raw }\end{array}$ & $\begin{array}{l}\text { ALinden_141119_hMP_BS3 } \\
\text { gel_20.raw }\end{array}$ & $\begin{array}{l}\text { ALinden_281119_310120_h } \\
\text { MP_EDC_gel_23.raw }\end{array}$ \\
\hline $\begin{array}{l}\text { ALinden_121119_hMP } \\
\text { BS3_11b.raw }\end{array}$ & $\begin{array}{l}\text { ALinden_141119_hMP_BS3 } \\
\text { gel_21.raw }\end{array}$ & $\begin{array}{l}\text { ALinden_281119_310120_h } \\
\text { MP_EDC_gel3.raw }\end{array}$ \\
\hline $\begin{array}{l}\text { ALinden_121119_hMP } \\
\text { BS3_12.raw }\end{array}$ & $\begin{array}{l}\text { ALinden_141119_hMP_BS3 } \\
\text { gel_22.raw }\end{array}$ & $\begin{array}{l}\text { ALinden_281119_310120_h } \\
\text { MP_EDC_gel_4.raw }\end{array}$ \\
\hline $\begin{array}{l}\text { ALinden_121119_hMP } \\
\text { BS3_13.raw }\end{array}$ & $\begin{array}{l}\text { ALinden_141119_hMP_BS3 } \\
\text { gel_23.raw }\end{array}$ & $\begin{array}{l}\text { ALinden_281119_310120_h } \\
\text { MP_EDC_gel5.raw }\end{array}$ \\
\hline $\begin{array}{l}\text { ALinden_121119_hMP } \\
\text { BS3_14.raw }\end{array}$ & $\begin{array}{l}\text { ALinden_141119_hMP_BS3 } \\
\text { gel_3.raw }\end{array}$ & $\begin{array}{l}\text { ALinden_281119_310120_h } \\
\text { MP_EDC_gel_6.raw }\end{array}$ \\
\hline $\begin{array}{l}\text { ALinden_121119_hMP } \\
\text { BS3_5to7.raw }\end{array}$ & $\begin{array}{l}\text { ALinden_141119_hMP_BS3 } \\
\text { gel_4.raw }\end{array}$ & $\begin{array}{l}\text { ALinden_281119_310120_h } \\
\text { MP_EDC_gel_7.raw }\end{array}$ \\
\hline $\begin{array}{l}\text { ALinden_121119_hMP } \\
\text { BS3_5to7b.raw }\end{array}$ & $\begin{array}{l}\text { ALinden_141119_hMP_BS3 } \\
\text { gel_5.raw }\end{array}$ & $\begin{array}{l}\text { ALinden_281119_310120_h } \\
\text { MP_EDC_gel_8.raw }\end{array}$ \\
\hline $\begin{array}{l}\text { ALinden_121119_hMP } \\
\text { BS3_8.raw }\end{array}$ & $\begin{array}{l}\text { ALinden_141119_hMP_BS3 } \\
\text { gel_6.raw }\end{array}$ & $\begin{array}{l}\text { ALinden_281119_310120_h } \\
\text { MP_EDC_gel_9.raw }\end{array}$ \\
\hline $\begin{array}{l}\text { ALinden_121119_hMP } \\
\text { BS3_8b.raw }\end{array}$ & $\begin{array}{l}\text { ALinden_141119_hMP_BS3 } \\
\text { gel_7.raw }\end{array}$ & $\begin{array}{l}\text { ALinden_281119_hMP_EDC } \\
\text { 10.raw }\end{array}$ \\
\hline $\begin{array}{l}\text { ALinden_121119_hMP } \\
\text { BS3_9.raw }\end{array}$ & $\begin{array}{l}\text { ALinden_141119_hMP_BS3 } \\
\text { gel_8.raw }\end{array}$ & $\begin{array}{l}\text { ALinden_281119_hMP_EDC } \\
\text { _10b.raw }\end{array}$ \\
\hline $\begin{array}{l}\text { ALinden_121119_hMP } \\
\text { BS3_9b.raw }\end{array}$ & $\begin{array}{l}\text { ALinden_141119_hMP_BS3 } \\
\text { gel_9.raw }\end{array}$ & $\begin{array}{l}\text { ALinden_281119_hMP_EDC } \\
\text { 11.raw }\end{array}$ \\
\hline $\begin{array}{l}\text { ALinden_141119_hMP } \\
\text { BS3_12b.raw }\end{array}$ & $\begin{array}{l}\text { ALinden_281119_310120_h } \\
\text { MP_EDC_gel_1.raw }\end{array}$ & $\begin{array}{l}\text { ALinden_281119_hMP_EDC } \\
\text { _11b.raw }\end{array}$ \\
\hline $\begin{array}{l}\text { ALinden_141119_hMP } \\
\text { BS3_13̄b.raw }\end{array}$ & $\begin{array}{l}\text { ALinden_281119_310120_h } \\
\text { MP_EDC_gel_10.raw }\end{array}$ & $\begin{array}{l}\text { ALinden_281119_hMP_EDC } \\
\text { 12.raw }\end{array}$ \\
\hline
\end{tabular}




\begin{tabular}{|l|l|l|}
\hline ALinden_141119_hMP & ALinden_281119_310120_h & ALinden_281119_hMP_EDC \\
BS3_14b.raw & MP_EDC_gel_11.raw & 12b.raw \\
\hline ALinden_141119_hMP & ALinden_281119_310120_h & ALinden_281119_hMP_EDC \\
BS3_gel_1.raw & MP_EDC_gel_12.raw & 13.raw \\
\hline ALinden_141119_hMP & ALinden_281119_310120_h & ALinden_281119_hMP_EDC \\
BS3_gel_10.raw & MP_EDC_gel_13.raw & 13b.raw \\
\hline ALinden_141119_hMP & ALinden_281119_310120_h & ALinden_281119_hMP_EDC \\
BS3_gel_11.raw & MP_EDC_gel_14.raw & 14.raw \\
\hline ALinden_141119_hMP & ALinden_281119_310120_h & ALinden_281119_hMP_EDC \\
BS3_gel_12.raw & MP_EDC_gel_15.raw & 14b.raw \\
\hline ALinden_141119_hMP & ALinden_281119_310120_h & ALinden_281119_hMP_EDC \\
BS3_gel_13.raw & MP_EDC_gel_16.raw & 15.raw \\
\hline ALinden_141119_hMP & ALinden_281119_310120_h & ALinden_281119_hMP_EDC \\
BS3_gel_14.raw & MP_EDC_gel_17.raw & 15b.raw \\
\hline ALinden_141119_hMP & ALinden_281119_310120_h & ALinden_2811119_hMP_EDC \\
BS3_gel_15.raw & MP_EDC_gel_18.raw & 7to8.raw \\
\hline ALinden_141119_hMP & ALinden_281119_310120_h & ALinden_281119_hMP_EDC \\
BS3_gel_16.raw & MP_EDC_gel_19.raw & 7to8b.raw \\
\hline ALinden_141119_hMP & ALinden_281119_310120_h & ALinden_281119_hMP_EDC \\
BS3_gel_17.raw & MP_EDC_gel_2.raw & 9.raw \\
\hline ALinden_141119_hMP & ALinden_281119_310120_h & ALinden_281119_hMP_EDC \\
BS3_gel_18.raw & MP_EDC_gel_20.raw & 9b.raw \\
\hline
\end{tabular}

Supplementary Table 15: Raw files acquired and used for analysis of Min8 immunoprecipitation after oxidative $\mathrm{Cu}(\mathrm{II})$-cross-linking.

\begin{tabular}{|l|l|l|}
\hline A_Linden_MDeckers_0702 & A_Linden_MDeckers_0702 & A_Linden_MDeckers_0702 \\
19_Min8-FLAG-IP_Cu- & 19_Min8-FLAG-IP_Cu- & 19_Min8-FLAG-IP_Cu- \\
XL_Ctrl1.raw & XL_XL3.raw & XL_XL6.raw \\
\hline A_Linden_MDeckers_0702 & A_Linden_MDeckers_0702 & A_Linden_MDeckers_0702 \\
19_Min8-FLAG-IP_Cu- & 19_Min8-FLAG-IP_Cu- & 19_Min8-FLAG-IP_Cu- \\
XL_Ctrl2.raw & XL_XL4.raw & XL_XL7.raw \\
\hline A_Linden_MDeckers_0702 & A_Linden_MDeckers_0702 & A_Linden_MDeckers_0702 \\
19_Min8-FLAG-IP_Cu- & 19__Min8-FLAG-IP_Cu- & 19_Min8-FLAG-IP_Cu- \\
XL_XL1.raw & XL_XL5.raw & XL_XL8.raw \\
\hline A_Linden_MDeckers_070219_Min8-FLAG-IP_Cu-XL_XL2.raw \\
\hline
\end{tabular}




\section{References}

1 Altmann, K., Durr, M. \& Westermann, B. Saccharomyces cerevisiae as a model organism to study mitochondrial biology: general considerations and basic procedures. Methods Mol Biol 372, 81-90 (2007).

2 Biddick, R. \& Young, E. T. The disorderly study of ordered recruitment. Yeast 26, 205-220 (2009).

3 Brocard-Masson, C. \& Dumas, B. The Fascinating World of Steroids:S. cerevisiaeas a Model Organism for the Study of Hydrocortisone Biosynthesis. Biotechnology and Genetic Engineering Reviews 22, 213-252 (2006).

4 Murakami, C. \& Kaeberlein, M. Quantifying Yeast Chronological Life Span by Outgrowth of Aged Cells. Journal of Visualized Experiments (2009).

5 Owsianowski, E., Walter, D. \& Fahrenkrog, B. Negative regulation of apoptosis in yeast. Biochimica et Biophysica Acta (BBA) - Molecular Cell Research 1783, 1303-1310 (2008).

$6 \quad$ Miller-Fleming, L., Giorgini, F. \& Outeiro, T. F. Yeast as a model for studying human neurodegenerative disorders. Biotechnology Journal 3, 325-338 (2008).

7 Foury, F. Human genetic diseases: a cross-talk between man and yeast. Gene 195, 1-10 (1997).

8 Gruhler, A., Olsen, J. V., Mohammed, S., Mortensen, P., Færgeman, N. J., Mann, M. \& Jensen, O. N. Quantitative Phosphoproteomics Applied to the Yeast Pheromone Signaling Pathway. Molecular \& Cellular Proteomics 4, 310-327 (2005).

9 Dannenmaier, S., Stiller, S. B., Morgenstern, M., Lubbert, P., Oeljeklaus, S., Wiedemann, N. \& Warscheid, B. Complete Native Stable Isotope Labeling by Amino Acids of Saccharomyces cerevisiae for Global Proteomic Analysis. Anal Chem 90, 10501-10509 (2018).

10 Murphy, J. P., Stepanova, E., Everley, R. A., Paulo, J. A. \& Gygi, S. P. Comprehensive Temporal Protein Dynamics during the Diauxic Shift inSaccharomyces cerevisiae. Molecular \& Cellular Proteomics 14, 2454-2465 (2015).

11 Schwikowski, B., Uetz, P. \& Fields, S. A network of protein-protein interactions in yeast. Nature Biotechnology 18, 1257-1261 (2000).

12 Scheffler, I. E. A century of mitochondrial research: achievements and perspectives. Mitochondrion 1, 3-31 (2001).

13 Chinnery, P. F. in GeneReviews $((R))$ (eds M. P. Adam, H. H. Ardinger, R. A. Pagon, S. E. Wallace, L. J. H. Bean, K. Stephens, \& A. Amemiya) (1993).

14 Onyango, I. G., Khan, S. M. \& Bennett, J. P., Jr. Mitochondria in the pathophysiology of Alzheimer's and Parkinson's diseases. Front Biosci (Landmark Ed) 22, 854-872 (2017).

15 Sagan, L. On the origin of mitosing cells. J Theor Biol 14, 255-274 (1967).

16 von der Malsburg, K., Müller, Judith M., Bohnert, M., Oeljeklaus, S., Kwiatkowska, P., Becker, T., Loniewska-Lwowska, A., Wiese, S., Rao, S., Milenkovic, D., Hutu, Dana P., Zerbes, Ralf M., Schulze-Specking, A., Meyer, Helmut E., Martinou, J.-C., Rospert, S., Rehling, P., Meisinger, C., Veenhuis, M., Warscheid, B., van der Klei, Ida J., Pfanner, N., Chacinska, A. \& van der Laan, M. Dual Role of Mitofilin in Mitochondrial Membrane Organization and Protein Biogenesis. Developmental Cell 21, 694-707 (2011).

17 Harner, M., Korner, C., Walther, D., Mokranjac, D., Kaesmacher, J., Welsch, U., Griffith, J., Mann, M., Reggiori, F. \& Neupert, W. The mitochondrial contact site complex, a determinant of mitochondrial architecture. EMBO J 30, 4356-4370 (2011). 
18 Arnold, I., Pfeiffer, K., Neupert, W., Stuart, R. A. \& Schagger, H. Yeast mitochondrial F1F0-ATP synthase exists as a dimer: identification of three dimerspecific subunits. EMBO J 17, 7170-7178 (1998).

19 Eydt, K., Davis, K. M., Behrendt, C., Wittig, I. \& Reichert, A. S. Cristae architecture is determined by an interplay of the MICOS complex and the F1Fo ATP synthase via Mic27 and Mic10. Microbial Cell 4, 259-272 (2017).

20 Paumard, P., Vaillier, J., Coulary, B., Schaeffer, J., Soubannier, V., Mueller, D. M., Brethes, D., di Rago, J. P. \& Velours, J. The ATP synthase is involved in generating mitochondrial cristae morphology. EMBO J 21, 221-230 (2002).

21 Pfanner, N., Warscheid, B. \& Wiedemann, N. Mitochondrial proteins: from biogenesis to functional networks. Nat Rev Mol Cell Biol 20, 267-284 (2019). Lill, R. Function and biogenesis of iron-sulphur proteins. Nature 460, 831-838 (2009).

23 Martinou, J. C. \& Youle, R. J. Mitochondria in apoptosis: Bcl-2 family members and mitochondrial dynamics. Dev Cell 21, 92-101 (2011).

24 de Zamaroczy, M. \& Bernardi, G. Sequence organization of the mitochondrial genome of yeast--a review. Gene 37, 1-17 (1985).

25 Anderson, S., Bankier, A. T., Barrell, B. G., de Bruijn, M. H., Coulson, A. R., Drouin, J., Eperon, I. C., Nierlich, D. P., Roe, B. A., Sanger, F., Schreier, P. H., Smith, A. J., Staden, R. \& Young, I. G. Sequence and organization of the human mitochondrial genome. Nature 290, 457-465 (1981).

26 O'Brien, T. W. \& Kalf, G. F. Ribosomes from rat liver mitochondria. I. Isolation procedure and contamination studies. J Biol Chem 242, 2172-2179 (1967).

27 Grivell, L. A., Reijnders, L. \& Borst, P. Isolation of yeast mitochondrial ribosomes highly active in protein synthesis. Biochim Biophys Acta 247, 91-103 (1971). De Silva, D., Tu, Y.-T., Amunts, A., Fontanesi, F. \& Barrientos, A. Mitochondrial ribosome assembly in health and disease. Cell Cycle 14, 2226-2250 (2015).

29 Cherry, J. M., Hong, E. L., Amundsen, C., Balakrishnan, R., Binkley, G., Chan, E. T., Christie, K. R., Costanzo, M. C., Dwight, S. S., Engel, S. R., Fisk, D. G., Hirschman, J. E., Hitz, B. C., Karra, K., Krieger, C. J., Miyasato, S. R., Nash, R. S., Park, J., Skrzypek, M. S., Simison, M., Weng, S. \& Wong, E. D. Saccharomyces Genome Database: the genomics resource of budding yeast. Nucleic Acids Res 40, D700-705 (2012).

30 Calvo, S. E. \& Mootha, V. K. The mitochondrial proteome and human disease. Annu Rev Genomics Hum Genet 11, 25-44 (2010).

31 Neupert, W. \& Herrmann, J. M. Translocation of Proteins into Mitochondria. Annual Review of Biochemistry 76, 723-749 (2007).

32 Wiedemann, N. \& Pfanner, N. Mitochondrial Machineries for Protein Import and Assembly. Annual Review of Biochemistry 86, 685-714 (2017).

33 Westermann, B. Mitochondrial fusion and fission in cell life and death. Nature Reviews Molecular Cell Biology 11, 872-884 (2010).

34 Wai, T. \& Langer, T. Mitochondrial Dynamics and Metabolic Regulation. Trends Endocrinol Metab 27, 105-117 (2016).

35 Egner, A., Jakobs, S. \& Hell, S. W. Fast 100-nm resolution three-dimensional microscope reveals structural plasticity of mitochondria in live yeast. Proceedings of the National Academy of Sciences 99, 3370-3375 (2002).

36 Chakraborty, A., Lyonnais, S., Battistini, F., Hospital, A., Medici, G., Prohens, R., Orozco, M., Vilardell, J. \& Sola, M. DNA structure directs positioning of the mitochondrial genome packaging protein Abf2p. Nucleic Acids Res 45, 951-967 (2017).

37 Foury, F., Roganti, T., Lecrenier, N. \& Purnelle, B. The complete sequence of the mitochondrial genome of Saccharomyces cerevisiae. FEBS Lett 440, 325-331 (1998). 
38 Capt, C., Passamonti, M. \& Breton, S. The human mitochondrial genome may code for more than 13 proteins. Mitochondrial DNA A DNA Mapp Seq Anal 27, 3098-3101 (2016).

39 Maleszka, R., Skelly, P. J. \& Clark-Walker, G. D. Rolling circle replication of DNA in yeast mitochondria. EMBO J 10, 3923-3929 (1991).

40 Diffley, J. F. \& Stillman, B. A close relative of the nuclear, chromosomal highmobility group protein HMG1 in yeast mitochondria. Proc Natl Acad Sci U S A 88, 7864-7868 (1991).

41 Ingles, C. J., Himmelfarb, H. J., Shales, M., Greenleaf, A. L. \& Friesen, J. D. Identification, molecular cloning, and mutagenesis of Saccharomyces cerevisiae RNA polymerase genes. Proc Natl Acad Sci U S A 81, 2157-2161 (1984).

42 Sanchez-Sandoval, E., Diaz-Quezada, C., Velazquez, G., Arroyo-Navarro, L. F., Almanza-Martinez, N., Trasvina-Arenas, C. H. \& Brieba, L. G. Yeast mitochondrial RNA polymerase primes mitochondrial DNA polymerase at origins of replication and promoter sequences. Mitochondrion 24, 22-31 (2015).

43 Alexeyev, M., Shokolenko, I., Wilson, G. \& LeDoux, S. The Maintenance of Mitochondrial DNA Integrity--Critical Analysis and Update. Cold Spring Harbor Perspectives in Biology 5, a012641-a012641 (2013).

44 Omar García-Lepe, U. \& Ma Bermúdez-Cruz, R. in DNA Repair- An Update Ch. Chapter 3, (2019).

45 Chen, X. J., Wang, X., Kaufman, B. A. \& Butow, R. A. Aconitase couples metabolic regulation to mitochondrial DNA maintenance. Science 307, 714-717 (2005).

46 Zelenaya-Troitskaya, O., Perlman, P. S. \& Butow, R. A. An enzyme in yeast mitochondria that catalyzes a step in branched-chain amino acid biosynthesis also functions in mitochondrial DNA stability. EMBO J 14, 3268-3276 (1995).

47 Contamine, V. \& Picard, M. Maintenance and integrity of the mitochondrial genome: a plethora of nuclear genes in the budding yeast. Microbiol Mol Biol Rev 64, 281-315 (2000).

48 Gray, M. W. Mitochondrial Evolution. Science 283, 1476-1481 (1999).

49 Neupert, W. A Perspective on Transport of Proteins into Mitochondria: A Myriad of Open Questions. Journal of Molecular Biology 427, 1135-1158 (2015).

50 Vögtle, F. N., Wortelkamp, S., Zahedi, R. P., Becker, D., Leidhold, C., Gevaert, K., Kellermann, J., Voos, W., Sickmann, A., Pfanner, N. \& Meisinger, C. Global Analysis of the Mitochondrial N-Proteome Identifies a Processing Peptidase Critical for Protein Stability. Cell 139, 428-439 (2009).

51 Dudek, J., Rehling, P. \& van der Laan, M. Mitochondrial protein import: Common principles and physiological networks. Biochimica et Biophysica Acta (BBA) Molecular Cell Research 1833, 274-285 (2013).

52 Araiso, Y., Tsutsumi, A., Qiu, J., Imai, K., Shiota, T., Song, J., Lindau, C., Wenz, L. S., Sakaue, H., Yunoki, K., Kawano, S., Suzuki, J., Wischnewski, M., Schutze, C., Ariyama, H., Ando, T., Becker, T., Lithgow, T., Wiedemann, N., Pfanner, N., Kikkawa, M. \& Endo, T. Structure of the mitochondrial import gate reveals distinct preprotein paths. Nature 575, 395-401 (2019).

53 Paschen, S. A., Waizenegger, T., Stan, T., Preuss, M., Cyrklaff, M., Hell, K., Rapaport, D. \& Neupert, W. Evolutionary conservation of biogenesis of betabarrel membrane proteins. Nature 426, 862-866 (2003).

54 Wiedemann, N., Kozjak, V., Chacinska, A., Schonfisch, B., Rospert, S., Ryan, M. T., Pfanner, N. \& Meisinger, C. Machinery for protein sorting and assembly in the mitochondrial outer membrane. Nature 424, 565-571 (2003).

55 Vasiljev, A., Ahting, U., Nargang, F. E., Go, N. E., Habib, S. J., Kozany, C., Panneels, V., Sinning, I., Prokisch, H., Neupert, W., Nussberger, S. \& Rapaport, D. Reconstituted TOM Core Complex and Tim9/Tim10 Complex of Mitochondria Are Sufficient for Translocation of the ADP/ATP Carrier across Membranes. Molecular Biology of the Cell 15, 1445-1458 (2004). 
56 Becker, T., Pfannschmidt, S., Guiard, B., Stojanovski, D., Milenkovic, D., Kutik, S., Pfanner, N., Meisinger, C. \& Wiedemann, N. Biogenesis of the Mitochondrial TOM Complex. Journal of Biological Chemistry 283, 120-127 (2008).

57 Popov-Čeleketić, J., Waizenegger, T. \& Rapaport, D. Mim1 Functions in an Oligomeric Form to Facilitate the Integration of Tom20 into the Mitochondrial Outer Membrane. Journal of Molecular Biology 376, 671-680 (2008).

58 Mesecke, N., Terziyska, N., Kozany, C., Baumann, F., Neupert, W., Hell, K. \& Herrmann, J. M. A Disulfide Relay System in the Intermembrane Space of Mitochondria that Mediates Protein Import. Cell 121, 1059-1069 (2005).

59 Sirrenberg, C., Endres, M., Folsch, H., Stuart, R. A., Neupert, W. \& Brunner, M. Carrier protein import into mitochondria mediated by the intermembrane proteins Tim10/Mrs11 and Tim12/Mrs5. Nature 391, 912-915 (1998).

60 Sirrenberg, C., Bauer, M. F., Guiard, B., Neupert, W. \& Brunner, M. Import of carrier proteins into the mitochondrial inner membrane mediated by Tim22.

Nature 384, 582-585 (1996).

61 Rehling, P. Protein Insertion into the Mitochondrial Inner Membrane by a TwinPore Translocase. Science 299, 1747-1751 (2003).

62 Okamoto, H., Miyagawa, A., Shiota, T., Tamura, Y. \& Endo, T. Intramolecular Disulfide Bond of Tim22 Protein Maintains Integrity of the TIM22 Complex in the Mitochondrial Inner Membrane. Journal of Biological Chemistry 289, 4827-4838 (2014).

63 Gomkale, R., Cruz-Zaragoza, L. D., Suppanz, I., Guiard, B., Montoya, J., Callegari, S., Pacheu-Grau, D., Warscheid, B. \& Rehling, P. Defining the Substrate Spectrum of the TIM22 Complex Identifies Pyruvate Carrier Subunits as Unconventional Cargos. Current Biology 30, 1119-1127.e1115 (2020).

64 Kinnally, K. W., Jensen, R. E. \& Lohret, T. A. Tim23, a Protein Import Component of the Mitochondrial Inner Membrane, Is Required for Normal Activity of the Multiple Conductance Channel, MCC. Journal of Cell Biology 137, 377-386 (1997).

65 Bauer, M. F., Sirrenberg, C., Neupert, W. \& Brunner, M. Role of Tim23 as Voltage Sensor and Presequence Receptor in Protein Import into Mitochondria. Cell 87, 33-41 (1996).

66 Albrecht, R., Rehling, P., Chacinska, A., Brix, J., Cadamuro, S. A., Volkmer, R., Guiard, B., Pfanner, N. \& Zeth, K. The Tim21 binding domain connects the preprotein translocases of both mitochondrial membranes. EMBO reports 7 , 1233-1238 (2006).

67 Chacinska, A., van der Laan, M., Mehnert, C. S., Guiard, B., Mick, D. U., Hutu, D. P., Truscott, K. N., Wiedemann, N., Meisinger, C., Pfanner, N. \& Rehling, P. Distinct Forms of Mitochondrial TOM-TIM Supercomplexes Define SignalDependent States of Preprotein Sorting. Molecular and Cellular Biology 30, 307318 (2010).

68 Truscott, K. N., Kovermann, P., Geissler, A., Merlin, A., Meijer, M., Driessen, A. J., Rassow, J., Pfanner, N. \& Wagner, R. A presequence- and voltage-sensitive channel of the mitochondrial preprotein translocase formed by Tim23. Nat Struct Biol 8, 1074-1082 (2001).

69 Denkert, N., Schendzielorz, A. B., Barbot, M., Versemann, L., Richter, F., Rehling, P. \& Meinecke, M. Cation selectivity of the presequence translocase channel Tim23 is crucial for efficient protein import. eLife 6 (2017).

70 Schulz, C. \& Rehling, P. Remodelling of the active presequence translocase drives motor-dependent mitochondrial protein translocation. Nat Commun 5, 4349 (2014).

71 Rehling, P., van der Laan, M., leva, R., Guiard, B., Clancy, A., Lytovchenko, O., Schulz, C. \& Schendzielorz, A. B. Two distinct membrane potential-dependent steps drive mitochondrial matrix protein translocation. Journal of Cell Biology 216, 83-92 (2017). 
72 Morgenstern, M., Stiller, S. B., Lubbert, P., Peikert, C. D., Dannenmaier, S., Drepper, F., Weill, U., Hoss, P., Feuerstein, R., Gebert, M., Bohnert, M., van der Laan, M., Schuldiner, M., Schutze, C., Oeljeklaus, S., Pfanner, N., Wiedemann, N. \& Warscheid, B. Definition of a High-Confidence Mitochondrial Proteome at Quantitative Scale. Cell Rep 19, 2836-2852 (2017).

73 Crabtree, H. G. Observations on the carbohydrate metabolism of tumours. Biochem J 23, 536-545 (1929).

74 Pronk, J. T., Yde Steensma, H. \& Van Dijken, J. P. Pyruvate metabolism in Saccharomyces cerevisiae. Yeast 12, 1607-1633 (1996).

75 Entian, K. D. Glucose repression: a complex regulatory system in yeast. Microbiol Sci 3, 366-371 (1986).

76 Gancedo, J. M. Carbon catabolite repression in yeast. Eur J Biochem 206, 297313 (1992).

77 Kayikci, Ö., Nielsen, J. \& Bolotin-Fukuhara, M. Glucose repression inSaccharomyces cerevisiae. FEMS Yeast Research 15 (2015).

78 Xiberras, J., Klein, M. \& Nevoigt, E. Glycerol as a substrate for Saccharomyces cerevisiae based bioprocesses - Knowledge gaps regarding the central carbon catabolism of this 'non-fermentable' carbon source. Biotechnology Advances 37 (2019).

79 Klein, M., Swinnen, S., Thevelein, J. M. \& Nevoigt, E. Glycerol metabolism and transport in yeast and fungi: established knowledge and ambiguities. Environ Microbiol 19, 878-893 (2017).

80 Boubekeur, S., Bunoust, O., Camougrand, N., Castroviejo, M., Rigoulet, M. \& Guérin, B. A Mitochondrial Pyruvate Dehydrogenase Bypass in the YeastSaccharomyces cerevisiae. Journal of Biological Chemistry 274, 2104421048 (1999).

81 Mitchell, P. Coupling of Phosphorylation to Electron and Hydrogen Transfer by a Chemi-Osmotic type of Mechanism. Nature 191, 144-148 (1961).

82 Saraste, M. Oxidative Phosphorylation at the fin de si\&egrave;cle. Science 283, 1488-1493 (1999).

83 Grandier-Vazeille, X., Bathany, K., Chaignepain, S., Camougrand, N., Manon, S. \& Schmitter, J. M. Yeast mitochondrial dehydrogenases are associated in a supramolecular complex. Biochemistry 40, 9758-9769 (2001).

84 Lenaz, G. \& Genova, M. L. Kinetics of integrated electron transfer in the mitochondrial respiratory chain: random collisions vs. solid state electron channeling. American Journal of Physiology-Cell Physiology 292, C1221-C1239 (2007).

85 Stuart, R. A. Supercomplex organization of the oxidative phosphorylation enzymes in yeast mitochondria. J Bioenerg Biomembr 40, 411-417 (2008).

86 Hackenbrock, C. R., Chazotte, B. \& Gupte, S. S. The random collision model and a critical assessment of diffusion and collision in mitochondrial electron transport. Journal of Bioenergetics and Biomembranes 18, 331-368 (1986).

87 Schagger, H. \& Pfeiffer, K. Supercomplexes in the respiratory chains of yeast and mammalian mitochondria. EMBO J 19, 1777-1783 (2000).

88 Cruciat, C. M., Brunner, S., Baumann, F., Neupert, W. \& Stuart, R. A. The cytochrome bc1 and cytochrome c oxidase complexes associate to form a single supracomplex in yeast mitochondria. J Biol Chem 275, 18093-18098 (2000). Acin-Perez, R., Fernandez-Silva, P., Peleato, M. L., Perez-Martos, A. \& Enriquez, J. A. Respiratory active mitochondrial supercomplexes. Mol Cell 32, 529-539 (2008).

90 Guo, R., Zong, S., Wu, M., Gu, J. \& Yang, M. Architecture of Human Mitochondrial Respiratory Megacomplex I2IIIIV2. Cell 170, 1247-1257 e1212 (2017). 
91 Hartley, A. M., Lukoyanova, N., Zhang, Y., Cabrera-Orefice, A., Arnold, S., Meunier, B., Pinotsis, N. \& Marechal, A. Structure of yeast cytochrome c oxidase in a supercomplex with cytochrome bc1. Nat Struct Mol Biol 26, 78-83 (2019).

92 Wu, M., Gu, J., Guo, R., Huang, Y. \& Yang, M. Structure of Mammalian Respiratory Supercomplex I1III2IV1. Cell 167, 1598-1609 e1510 (2016).

93 Mileykovskaya, E., Penczek, P. A., Fang, J., Mallampalli, V. K., Sparagna, G. C. \& Dowhan, W. Arrangement of the respiratory chain complexes in Saccharomyces cerevisiae supercomplex III2IV2 revealed by single particle cryoelectron microscopy. J Biol Chem 287, 23095-23103 (2012).

94 Wittig, I., Carrozzo, R., Santorelli, F. M. \& Schägger, H. Supercomplexes and subcomplexes of mitochondrial oxidative phosphorylation. Biochimica et Biophysica Acta (BBA) - Bioenergetics 1757, 1066-1072 (2006). van der Laan, M., Wiedemann, N., Mick, D. U., Guiard, B., Rehling, P. \& Pfanner, N. A Role for Tim21 in Membrane-Potential-Dependent Preprotein Sorting in Mitochondria. Current Biology 16, 2271-2276 (2006).

96 Pfanner, N., Rehling, P., Hutu, D. P., van der Laan, M. \& Wiedemann, N. Sorting switch of mitochondrial presequence translocase involves coupling of motor module to respiratory chain. Journal of Cell Biology 179, 1115-1122 (2007).

97 Dienhart, M. K. \& Stuart, R. A. The yeast Aac2 protein exists in physical association with the cytochrome bc1-COX supercomplex and the TIM23 machinery. Mol Biol Cell 19, 3934-3943 (2008).

98 Claypool, S. M., Oktay, Y., Boontheung, P., Loo, J. A. \& Koehler, C. M. Cardiolipin defines the interactome of the major ADP/ATP carrier protein of the mitochondrial inner membrane. J Cell Biol 182, 937-950 (2008).

99 Schweppe, D. K., Chavez, J. D., Lee, C. F., Caudal, A., Kruse, S. E., Stuppard, R., Marcinek, D. J., Shadel, G. S., Tian, R. \& Bruce, J. E. Mitochondrial protein interactome elucidated by chemical cross-linking mass spectrometry. Proc Natl Acad Sci U S A 114, 1732-1737 (2017).

100 Liu, F., Lossl, P., Rabbitts, B. M., Balaban, R. S. \& Heck, A. J. R. The interactome of intact mitochondria by cross-linking mass spectrometry provides evidence for coexisting respiratory supercomplexes. Mol Cell Proteomics 17, 216-232 (2018).

101 Ryl, P. S. J., Bohlke-Schneider, M., Lenz, S., Fischer, L., Budzinski, L., Stuiver, M., Mendes, M. M. L., Sinn, L., O'Reilly, F. J. \& Rappsilber, J. In Situ Structural Restraints from Crosslinking Mass Spectrometry in Human Mitochondria. $J$ Proteome Res 19, 327-336, (2019).

102 Makepeace, K. A. T., Mohammed, Y., Rudashevskaya, E. L., Petrotchenko, E. V., Voegtle, F. N., Meisinger, C., Sickmann, A. \& Borchers, C. H. Improving identification of in-organello protein-protein interactions using an affinityenrichable, isotopically-coded, and mass spectrometry-cleavable chemical crosslinker. Mol Cell Proteomics 19, 624-639 (2020).

103 Acin-Perez, R. \& Enriquez, J. A. The function of the respiratory supercomplexes: The plasticity model. Biochimica et Biophysica Acta (BBA) - Bioenergetics 1837, 444-450 (2014).

104 Lapuente-Brun, E., Moreno-Loshuertos, R., Acín-Pérez, R., Latorre-Pellicer, A., Colás, C., Balsa, E., Perales-Clemente, E., Quirós, P. M., Calvo, E., RodríguezHernández, M. A., Navas, P., Cruz, R., Carracedo, Á., López-Otín, C., PérezMartos, A., Fernández-Silva, P., Fernández-Vizarra, E. \& Enríquez, J. A. Supercomplex Assembly Determines Electron Flux in the Mitochondrial Electron Transport Chain. Science 340, 1567-1570 (2013).

105 Boumans, H., Grivell, L. A. \& Berden, J. A. The respiratory chain in yeast behaves as a single functional unit. J Biol Chem 273, $4872-4877$ (1998).

106 Gupte, S. S. \& Hackenbrock, C. R. The role of cytochrome c diffusion in mitochondrial electron transport. J Biol Chem 263, 5248-5253 (1988). 
107 Acín-Pérez, R., Bayona-Bafaluy, M. a. P., Fernández-Silva, P., MorenoLoshuertos, R., Pérez-Martos, A., Bruno, C., Moraes, C. T. \& Enríquez, J. A. Respiratory Complex III Is Required to Maintain Complex I in Mammalian Mitochondria. Molecular Cell 13, 805-815 (2004).

108 Guerrero-Castillo, S., Baertling, F., Kownatzki, D., Wessels, H. J., Arnold, S., Brandt, U. \& Nijtmans, L. The Assembly Pathway of Mitochondrial Respiratory Chain Complex I. Cell Metabolism 25, 128-139 (2017).

109 Boveris, A. Mitochondrial production of superoxide radical and hydrogen peroxide. Adv Exp Med Biol 78, 67-82 (1977).

110 Wong, G. H. W., Elwell, J. H., Oberley, L. W. \& Goeddel, D. V. Manganous superoxide dismutase is essential for cellular resistance to cytotoxicity of tumor necrosis factor. Cell 58, 923-931 (1989).

111 Zhang, M., Mileykovskaya, E. \& Dowhan, W. Gluing the Respiratory Chain Together. Journal of Biological Chemistry 277, 43553-43556 (2002).

112 Zhang, M., Mileykovskaya, E. \& Dowhan, W. Cardiolipin Is Essential for Organization of Complexes III and IV into a Supercomplex in Intact Yeast Mitochondria. Journal of Biological Chemistry 280, 29403-29408 (2005).

113 Mileykovskaya, E. \& Dowhan, W. Cardiolipin-dependent formation of mitochondrial respiratory supercomplexes. Chemistry and Physics of Lipids 179, 42-48 (2014).

114 Pfeiffer, K., Gohil, V., Stuart, R. A., Hunte, C., Brandt, U., Greenberg, M. L. \& Schägger, H. Cardiolipin Stabilizes Respiratory Chain Supercomplexes. Journal of Biological Chemistry 278, 52873-52880 (2003).

115 Vukotic, M., Oeljeklaus, S., Wiese, S., Vogtle, F. N., Meisinger, C., Meyer, H. E., Zieseniss, A., Katschinski, D. M., Jans, D. C., Jakobs, S., Warscheid, B., Rehling, P. \& Deckers, M. Rcf1 mediates cytochrome oxidase assembly and respirasome formation, revealing heterogeneity of the enzyme complex. Cell Metab 15, 336347 (2012).

116 Strogolova, V., Furness, A., Robb-McGrath, M., Garlich, J. \& Stuart, R. A. Rcf1 and Rcf2, members of the hypoxia-induced gene 1 protein family, are critical components of the mitochondrial cytochrome bc1-cytochrome c oxidase supercomplex. Mol Cell Biol 32, 1363-1373 (2012).

117 Chen, Y.-C., Taylor, Eric B., Dephoure, N., Heo, J.-M., Tonhato, A., Papandreou, I., Nath, N., Denko, Nicolas C., Gygi, Steven P. \& Rutter, J. Identification of a Protein Mediating Respiratory Supercomplex Stability. Cell Metabolism 15, 348360 (2012).

118 Garlich, J., Strecker, V., Wittig, I. \& Stuart, R. A. Mutational Analysis of the QRRQ Motif in the Yeast Hig1 Type 2 Protein Rcf1 Reveals a Regulatory Role for the CytochromecOxidase Complex. Journal of Biological Chemistry 292, 5216-5226 (2017).

119 Rompler, K., Muller, T., Juris, L., Wissel, M., Vukotic, M., Hofmann, K. \& Deckers, M. Overlapping Role of Respiratory Supercomplex Factor Rcf2 and Its N-terminal Homolog Rcf3 in Saccharomyces cerevisiae. J Biol Chem 291, 23769-23778 (2016).

120 Hartley, A. M., Meunier, B., Pinotsis, N. \& Maréchal, A. Rcf2 revealed in cryo-EM structures of hypoxic isoforms of mature mitochondrial III-IV supercomplexes. Proceedings of the National Academy of Sciences (2020).

121 Rak, M., Bénit, P., Chrétien, D., Bouchereau, J., Schiff, M., El-Khoury, R., Tzagoloff, A. \& Rustin, P. Mitochondrial cytochrome c oxidase deficiency. Clinical Science 130, 393-407 (2016).

122 Mick, D. U., Fox, T. D. \& Rehling, P. Inventory control: cytochrome c oxidase assembly regulates mitochondrial translation. Nat Rev Mol Cell Biol 12, 14-20 (2011). 
123 Soto, I. C., Fontanesi, F., Liu, J. \& Barrientos, A. Biogenesis and assembly of eukaryotic cytochrome c oxidase catalytic core. Biochimica et Biophysica Acta (BBA) - Bioenergetics 1817, 883-897 (2012).

124 Levchenko, M., Wuttke, J. M., Rompler, K., Schmidt, B., Neifer, K., Juris, L., Wissel, M., Rehling, P. \& Deckers, M. Cox26 is a novel stoichiometric subunit of the yeast cytochrome c oxidase. Biochim Biophys Acta 1863, 1624-1632 (2016).

125 Strecker, V., Kadeer, Z., Heidler, J., Cruciat, C. M., Angerer, H., Giese, H., Pfeiffer, K., Stuart, R. A. \& Wittig, I. Supercomplex-associated Cox26 protein binds to cytochrome c oxidase. Biochim Biophys Acta 1863, 1643-1652 (2016).

126 Fontanesi, F., Soto, I. C. \& Barrientos, A. Cytochrome c oxidase biogenesis: new levels of regulation. IUBMB Life 60, 557-568 (2008).

127 Stiburek, L., Vesela, K., Hansikova, H., Pecina, P., Tesarova, M., Cerna, L., Houstek, J. \& Zeman, J. Tissue-specific cytochrome c oxidase assembly defects due to mutations in SCO2 and SURF1. Biochem J 392, 625-632 (2005).

128 Horan, S., Bourges, I., Taanman, J. W. \& Meunier, B. Analysis of COX2 mutants reveals cytochrome oxidase subassemblies in yeast. Biochem J 390, 703-708 (2005).

129 Church, C., Goehring, B., Forsha, D., Wazny, P. \& Poyton, R. O. A role for Pet $100 p$ in the assembly of yeast cytochrome c oxidase: interaction with a subassembly that accumulates in a pet 100 mutant. J Biol Chem 280, 1854-1863 (2005).

130 Timón-Gómez, A., Nývltová, E., Abriata, L. A., Vila, A. J., Hosler, J. \& Barrientos, A. Mitochondrial cytochrome c oxidase biogenesis: Recent developments. Seminars in Cell \& Developmental Biology 76, 163-178 (2018).

131 Massa, V., Fernandez-Vizarra, E., Alshahwan, S., Bakhsh, E., Goffrini, P., Ferrero, I., Mereghetti, P., D'Adamo, P., Gasparini, P. \& Zeviani, M. Severe infantile encephalomyopathy caused by a mutation in COX6B1, a nucleusencoded subunit of cytochrome c oxidase. Am J Hum Genet 82, 1281-1289 (2008).

132 Taanman, J. W. \& Capaldi, R. A. Subunit Vla of yeast cytochrome c oxidase is not necessary for assembly of the enzyme complex but modulates the enzyme activity. Isolation and characterization of the nuclear-coded gene. $\mathrm{J}$ Biol Chem 268, 18754-18761 (1993).

133 Mick, D. U., Vukotic, M., Piechura, H., Meyer, H. E., Warscheid, B., Deckers, M. \& Rehling, P. Coa3 and Cox14 are essential for negative feedback regulation of COX1 translation in mitochondria. J Cell Biol 191, 141-154 (2010).

134 Mick, D. U., Wagner, K., van der Laan, M., Frazier, A. E., Perschil, I., Pawlas, M., Meyer, H. E., Warscheid, B. \& Rehling, P. Shy1 couples Cox1 translational regulation to cytochrome c oxidase assembly. EMBO J 26, 4347-4358 (2007).

135 Pierrel, F., Bestwick, M. L., Cobine, P. A., Khalimonchuk, O., Cricco, J. A. \& Winge, D. R. Coa1 links the Mss51 post-translational function to Cox1 cofactor insertion in cytochrome c oxidase assembly. EMBO J 26, 4335-4346 (2007).

136 Pierrel, F., Khalimonchuk, O., Cobine, P. A., Bestwick, M. \& Winge, D. R. Coa2 is an assembly factor for yeast cytochrome $c$ oxidase biogenesis that facilitates the maturation of Cox1. Mol Cell Biol 28, 4927-4939 (2008).

137 Wilkins, M. R., Sanchez, J.-C., Gooley, A. A., Appel, R. D., Humphery-Smith, I., Hochstrasser, D. F. \& Williams, K. L. Progress with Proteome Projects: Why all Proteins Expressed by a Genome Should be Identified and How To Do It. Biotechnology and Genetic Engineering Reviews 13, $19-50$ (1996).

138 Aebersold, R. \& Mann, M. Mass-spectrometric exploration of proteome structure and function. Nature 537, 347-355 (2016).

139 Sickmann, A., Reinders, J., Wagner, Y., Joppich, C., Zahedi, R., Meyer, H. E., Schonfisch, B., Perschil, I., Chacinska, A., Guiard, B., Rehling, P., Pfanner, N. \& Meisinger, C. The proteome of Saccharomyces cerevisiae mitochondria. Proc Natl Acad Sci U S A 100, 13207-13212 (2003). 
140 Reinders, J., Zahedi, R. P., Pfanner, N., Meisinger, C. \& Sickmann, A. Toward the Complete Yeast Mitochondrial Proteome: Multidimensional Separation Techniques for Mitochondrial Proteomics. Journal of Proteome Research 5, 1543-1554 (2006).

141 Gonczarowska-Jorge, H., Zahedi, R. P. \& Sickmann, A. The proteome of baker's yeast mitochondria. Mitochondrion 33, 15-21 (2017).

142 Vogtle, F. N., Burkhart, J. M., Gonczarowska-Jorge, H., Kucukkose, C., Taskin, A. A., Kopczynski, D., Ahrends, R., Mossmann, D., Sickmann, A., Zahedi, R. P. \& Meisinger, C. Landscape of submitochondrial protein distribution. Nat Commun 8, 290 (2017).

143 Vogtle, F. N., Burkhart, J. M., Rao, S., Gerbeth, C., Hinrichs, J., Martinou, J. C., Chacinska, A., Sickmann, A., Zahedi, R. P. \& Meisinger, C. Intermembrane space proteome of yeast mitochondria. Mol Cell Proteomics 11, 1840-1852 (2012).

144 Zahedi, R. P., Sickmann, A., Boehm, A. M., Winkler, C., Zufall, N., Schonfisch, B., Guiard, B., Pfanner, N. \& Meisinger, C. Proteomic analysis of the yeast mitochondrial outer membrane reveals accumulation of a subclass of preproteins. Mol Biol Cell 17, 1436-1450 (2006).

145 Di Bartolomeo, F., Malina, C., Campbell, K., Mormino, M., Fuchs, J., Vorontsov, E., Gustafsson, C. M. \& Nielsen, J. Absolute yeast mitochondrial proteome quantification reveals trade-off between biosynthesis and energy generation during diauxic shift. Proceedings of the National Academy of Sciences 117, 75247535 (2020).

146 Renvoise, M., Bonhomme, L., Davanture, M., Valot, B., Zivy, M. \& Lemaire, C. Quantitative variations of the mitochondrial proteome and phosphoproteome during fermentative and respiratory growth in Saccharomyces cerevisiae. $J$ Proteomics 106, 140-150 (2014).

147 Ohlmeier, S., Hiltunen, J. K. \& Bergmann, U. Protein phosphorylation in mitochondria --a study on fermentative and respiratory growth of Saccharomyces cerevisiae. Electrophoresis 31, 2869-2881 (2010).

148 Ohlmeier, S., Kastaniotis, A. J., Hiltunen, J. K. \& Bergmann, U. The yeast mitochondrial proteome, a study of fermentative and respiratory growth. $\mathrm{J} \mathrm{Biol}$ Chem 279, 3956-3979 (2004).

149 Steen, H. \& Mann, M. The abc's (and xyz's) of peptide sequencing. Nature Reviews Molecular Cell Biology 5, 699-711 (2004).

150 Washburn, M. P., Wolters, D. \& Yates, J. R. Large-scale analysis of the yeast proteome by multidimensional protein identification technology. Nature Biotechnology 19, 242-247 (2001).

151 Motoyama, A. \& Yates, J. R. Multidimensional LC Separations in Shotgun Proteomics. Analytical Chemistry 80, 7187-7193 (2008).

152 Fenn, J., Mann, M., Meng, C., Wong, S. \& Whitehouse, C. Electrospray ionization for mass spectrometry of large biomolecules. Science 246, 64-71 (1989).

153 Hu, Q., Noll, R. J., Li, H., Makarov, A., Hardman, M. \& Graham Cooks, R. The Orbitrap: a new mass spectrometer. Journal of Mass Spectrometry 40, 430-443 (2005).

154 Michalski, A., Damoc, E., Hauschild, J.-P., Lange, O., Wieghaus, A., Makarov, A., Nagaraj, N., Cox, J., Mann, M. \& Horning, S. Mass Spectrometry-based Proteomics Using Q Exactive, a High-performance Benchtop Quadrupole Orbitrap Mass Spectrometer. Molecular \& Cellular Proteomics 10 (2011).

155 Hoffmann, E. d. \& Stroobant, V. Mass spectrometry : principles and applications. 2nd edn, (Wiley, 2001).

156 Makarov, A. Electrostatic Axially Harmonic Orbital Trapping: A HighPerformance Technique of Mass Analysis. Analytical Chemistry 72, 1156-1162 (2000). 
157 Wells, J. M. \& McLuckey, S. A. Collision-induced dissociation (CID) of peptides and proteins. Methods Enzymol 402, 148-185 (2005).

158 Olsen, J. V., Macek, B., Lange, O., Makarov, A., Horning, S. \& Mann, M. Higherenergy C-trap dissociation for peptide modification analysis. Nat Methods 4, 709712 (2007).

159 Roepstorff, P. \& Fohlman, J. Proposal for a common nomenclature for sequence ions in mass spectra of peptides. Biomed Mass Spectrom 11, 601 (1984).

160 Picotti, P. \& Aebersold, R. Selected reaction monitoring-based proteomics: workflows, potential, pitfalls and future directions. Nat Methods 9, 555-566 (2012).

161 Gillet, L. C., Navarro, P., Tate, S., Rost, H., Selevsek, N., Reiter, L., Bonner, R. \& Aebersold, R. Targeted data extraction of the MS/MS spectra generated by dataindependent acquisition: a new concept for consistent and accurate proteome analysis. Mol Cell Proteomics 11, 0111016717 (2012).

162 Venable, J. D., Dong, M. Q., Wohlschlegel, J., Dillin, A. \& Yates, J. R. Automated approach for quantitative analysis of complex peptide mixtures from tandem mass spectra. Nat Methods 1, 39-45 (2004).

163 Egertson, J. D., MacLean, B., Johnson, R., Xuan, Y. \& MacCoss, M. J. Multiplexed peptide analysis using data-independent acquisition and Skyline. Nat Protoc 10, 887-903 (2015).

164 Wolff, M. M. \& Stephens, W. E. A Pulsed Mass Spectrometer with Time Dispersion. Review of Scientific Instruments 24, 616-617 (1953).

165 Senko, M. W., Remes, P. M., Canterbury, J. D., Mathur, R., Song, Q., Eliuk, S. M., Mullen, C., Earley, L., Hardman, M., Blethrow, J. D., Bui, H., Specht, A., Lange, O., Denisov, E., Makarov, A., Horning, S. \& Zabrouskov, V. Novel Parallelized Quadrupole/Linear Ion Trap/Orbitrap Tribrid Mass Spectrometer Improving Proteome Coverage and Peptide Identification Rates. Analytical Chemistry 85, 11710-11714 (2013).

166 Eng, J. K., McCormack, A. L. \& Yates, J. R. An approach to correlate tandem mass spectral data of peptides with amino acid sequences in a protein database. J Am Soc Mass Spectrom 5, 976-989 (1994).

167 Perkins, D. N., Pappin, D. J., Creasy, D. M. \& Cottrell, J. S. Probability-based protein identification by searching sequence databases using mass spectrometry data. Electrophoresis 20, 3551-3567 (1999).

168 Käll, L., Storey, J. D., MacCoss, M. J. \& Noble, W. S. Assigning Significance to Peptides Identified by Tandem Mass Spectrometry Using Decoy Databases. Journal of Proteome Research 7, 29-34 (2008).

169 Elias, J. E. \& Gygi, S. P. Target-decoy search strategy for increased confidence in large-scale protein identifications by mass spectrometry. Nat Methods 4, 207214 (2007).

170 Bantscheff, M., Lemeer, S., Savitski, M. M. \& Kuster, B. Quantitative mass spectrometry in proteomics: critical review update from 2007 to the present. Anal Bioanal Chem 404, 939-965 (2012).

171 Ong, S. E. \& Mann, M. Stable isotope labeling by amino acids in cell culture for quantitative proteomics. Methods Mol Biol 359, 37-52 (2007).

172 Zanivan, S., Krueger, M. \& Mann, M. In vivo quantitative proteomics: the SILAC mouse. Methods Mol Biol 757, 435-450 (2012).

173 Oeljeklaus, S., Schummer, A., Suppanz, I. \& Warscheid, B. in Stable Isotope Labeling by Amino Acids in Cell Culture (SILAC) Methods in Molecular Biology Ch. Chapter 3, 23-46 (2014).

174 Thompson, A., Schafer, J., Kuhn, K., Kienle, S., Schwarz, J., Schmidt, G., Neumann, T., Johnstone, R., Mohammed, A. K. \& Hamon, C. Tandem mass tags: a novel quantification strategy for comparative analysis of complex protein mixtures by MS/MS. Anal Chem 75, 1895-1904 (2003). 
175 Ting, L., Rad, R., Gygi, S. P. \& Haas, W. MS3 eliminates ratio distortion in isobaric multiplexed quantitative proteomics. Nat Methods 8, 937-940 (2011).

176 Lazar, C., Gatto, L., Ferro, M., Bruley, C. \& Burger, T. Accounting for the Multiple Natures of Missing Values in Label-Free Quantitative Proteomics Data Sets to Compare Imputation Strategies. J Proteome Res 15, 1116-1125 (2016).

177 Webb-Robertson, B. J., Wiberg, H. K., Matzke, M. M., Brown, J. N., Wang, J., McDermott, J. E., Smith, R. D., Rodland, K. D., Metz, T. O., Pounds, J. G. \& Waters, K. M. Review, evaluation, and discussion of the challenges of missing value imputation for mass spectrometry-based label-free global proteomics. $J$ Proteome Res 14, 1993-2001 (2015).

178 Roux, K. J., Kim, D. I., Raida, M. \& Burke, B. A promiscuous biotin ligase fusion protein identifies proximal and interacting proteins in mammalian cells. $\mathrm{J}$ Cell Biol 196, 801-810 (2012).

179 Rhee, H. W., Zou, P., Udeshi, N. D., Martell, J. D., Mootha, V. K., Carr, S. A. \& Ting, A. Y. Proteomic Mapping of Mitochondria in Living Cells via Spatially Restricted Enzymatic Tagging. Science 339, 1328-1331 (2013).

180 Rappsilber, J. The beginning of a beautiful friendship: cross-linking/mass spectrometry and modelling of proteins and multi-protein complexes. J Struct Biol 173, 530-540 (2011).

181 Leitner, A., Faini, M., Stengel, F. \& Aebersold, R. Crosslinking and Mass Spectrometry: An Integrated Technology to Understand the Structure and Function of Molecular Machines. Trends in Biochemical Sciences 41, 20-32 (2016).

182 Schmidt, C. \& Urlaub, H. Combining cryo-electron microscopy (cryo-EM) and cross-linking mass spectrometry (CX-MS) for structural elucidation of large protein assemblies. Curr Opin Struct Biol 46, 157-168 (2017).

183 Steigenberger, B., Albanese, P., Heck, A. J. R. \& Scheltema, R. A. To Cleave or Not To Cleave in XL-MS? J Am Soc Mass Spectrom 31, 196-206 (2020).

184 Swaim, C. L., Smith, J. B. \& Smith, D. L. Unexpected products from the reaction of the synthetic cross-linker 3,3'-dithiobis(sulfosuccinimidyl propionate), DTSSP with peptides. J Am Soc Mass Spectrom 15, 736-749 (2004).

185 Leavell, M. D., Novak, P., Behrens, C. R., Schoeniger, J. S. \& Kruppa, G. H. Strategy for selective chemical cross-linking of tyrosine and lysine residues. J Am Soc Mass Spectrom 15, 1604-1611 (2004).

186 Kalkhof, S. \& Sinz, A. Chances and pitfalls of chemical cross-linking with aminereactive N-hydroxysuccinimide esters. Anal Bioanal Chem 392, 305-312 (2008).

187 Merkley, E. D., Rysavy, S., Kahraman, A., Hafen, R. P., Daggett, V. \& Adkins, J. $\mathrm{N}$. Distance restraints from crosslinking mass spectrometry: Mining a molecular dynamics simulation database to evaluate lysine-lysine distances. Protein Science 23, 747-759 (2014).

188 Paramelle, D., Miralles, G., Subra, G. \& Martinez, J. Chemical cross-linkers for protein structure studies by mass spectrometry. Proteomics 13, 438-456 (2013).

189 Novak, P. \& Kruppa, G. H. Intra-molecular cross-linking of acidic residues for protein structure studies. Eur J Mass Spectrom (Chichester) 14, 355-365 (2008).

190 Giese, S. H., Belsom, A. \& Rappsilber, J. Optimized Fragmentation Regime for Diazirine Photo-Cross-Linked Peptides. Analytical Chemistry 88, 8239-8247 (2016).

191 Kovalenko, O. V., Yang, X. H. \& Hemler, M. E. A Novel Cysteine Cross-linking Method Reveals a Direct Association between Claudin-1 and Tetraspanin CD9. Molecular \& Cellular Proteomics 6, 1855-1867 (2007).

192 Sinz, A. Chemical cross-linking and mass spectrometry for mapping threedimensional structures of proteins and protein complexes. Journal of Mass Spectrometry 38, 1225-1237 (2003).

193 Young, M. M., Tang, N., Hempel, J. C., Oshiro, C. M., Taylor, E. W., Kuntz, I. D., Gibson, B. W. \& Dollinger, G. High throughput protein fold identification by using 
experimental constraints derived from intramolecular cross-links and mass spectrometry. Proc Natl Acad Sci U S A 97, 5802-5806 (2000).

194 Rappsilber, J., Siniossoglou, S., Hurt, E. C. \& Mann, M. A generic strategy to analyze the spatial organization of multi-protein complexes by cross-linking and mass spectrometry. Anal Chem 72, 267-275 (2000).

195 Maiolica, A., Cittaro, D., Borsotti, D., Sennels, L., Ciferri, C., Tarricone, C., Musacchio, A. \& Rappsilber, J. Structural analysis of multiprotein complexes by cross-linking, mass spectrometry, and database searching. Mol Cell Proteomics 6, 2200-2211 (2007).

196 Chen, Z. A., Jawhari, A., Fischer, L., Buchen, C., Tahir, S., Kamenski, T., Rasmussen, M., Lariviere, L., Bukowski-Wills, J. C., Nilges, M., Cramer, P. \& Rappsilber, J. Architecture of the RNA polymerase II-TFIIF complex revealed by cross-linking and mass spectrometry. EMBO J 29, 717-726 (2010).

197 Agafonov, D. E., Kastner, B., Dybkov, O., Hofele, R. V., Liu, W. T., Urlaub, H., Luhrmann, R. \& Stark, H. Molecular architecture of the human U4/U6.U5 trisnRNP. Science 351, 1416-1420 (2016).

198 Bertram, K., Agafonov, D. E., Dybkov, O., Haselbach, D., Leelaram, M. N., Will, C. L., Urlaub, H., Kastner, B., Lührmann, R. \& Stark, H. Cryo-EM Structure of a Pre-catalytic Human Spliceosome Primed for Activation. Cell 170, 701-713.e711 (2017).

199 Plaschka, C., Larivière, L., Wenzeck, L., Seizl, M., Hemann, M., Tegunov, D., Petrotchenko, E. V., Borchers, C. H., Baumeister, W., Herzog, F., Villa, E. \& Cramer, P. Architecture of the RNA polymerase II-Mediator core initiation complex. Nature 518, 376-380 (2015).

200 Kosinski, J., Mosalaganti, S., von Appen, A., Teimer, R., DiGuilio, A. L., Wan, W., Bui, K. H., Hagen, W. J. H., Briggs, J. A. G., Glavy, J. S., Hurt, E. \& Beck, M. Molecular architecture of the inner ring scaffold of the human nuclear pore complex. Science 352, 363-365 (2016).

201 Russel, D., Lasker, K., Webb, B., Velázquez-Muriel, J., Tjioe, E., SchneidmanDuhovny, D., Peterson, B. \& Sali, A. Putting the Pieces Together: Integrative Modeling Platform Software for Structure Determination of Macromolecular Assemblies. PLoS Biology 10 (2012).

202 Dominguez, C., Boelens, R. \& Bonvin, A. M. J. J. HADDOCK: A Protein-Protein Docking Approach Based on Biochemical or Biophysical Information. Journal of the American Chemical Society 125, 1731-1737 (2003).

203 Fernandez-Fuentes, N., Kahraman, A., Herzog, F., Leitner, A., Rosenberger, G., Aebersold, R. \& Malmström, L. Cross-Link Guided Molecular Modeling with ROSETTA. PLOS ONE 8 (2013).

204 Herzog, F., Kahraman, A., Boehringer, D., Mak, R., Bracher, A., Walzthoeni, T., Leitner, A., Beck, M., Hartl, F. U., Ban, N., Malmstrom, L. \& Aebersold, R.

Structural probing of a protein phosphatase $2 \mathrm{~A}$ network by chemical cross-linking and mass spectrometry. Science 337, 1348-1352 (2012).

205 Goetze, M., lacobucci, C., Ihling, C. H. \& Sinz, A. A Simple Cross-Linking/Mass Spectrometry Workflow to Study System-Wide Protein Interactions. Anal Chem 91, 10236-10244 (2019).

206 Weisbrod, C. R., Chavez, J. D., Eng, J. K., Yang, L., Zheng, C. \& Bruce, J. E. In vivo protein interaction network identified with a novel real-time cross-linked peptide identification strategy. J Proteome Res 12, 1569-1579 (2013).

207 Zhang, H., Tang, X., Munske, G. R., Tolic, N., Anderson, G. A. \& Bruce, J. E. Identification of Protein-Protein Interactions and Topologies in Living Cells with Chemical Cross-linking and Mass Spectrometry. Molecular \& Cellular Proteomics 8, 409-420 (2009).

208 Chavez, J. D., Schweppe, D. K., Eng, J. K., Zheng, C., Taipale, A., Zhang, Y., Takara, K. \& Bruce, J. E. Quantitative interactome analysis reveals a chemoresistant edgotype. Nat Commun 6, 7928 (2015). 
209 Chavez, J. D., Weisbrod, C. R., Zheng, C., Eng, J. K. \& Bruce, J. E. Protein Interactions, Post-translational Modifications and Topologies in Human Cells. Molecular \& Cellular Proteomics 12, 1451-1467 (2013).

210 Chavez, J. D., Lee, C. F., Caudal, A., Keller, A., Tian, R. \& Bruce, J. E. Chemical Crosslinking Mass Spectrometry Analysis of Protein Conformations and Supercomplexes in Heart Tissue. Cell Systems 6, 136-141.e135 (2018).

211 Fürsch, J., Kammer, K. M., Kreft, S. G., Beck, M. \& Stengel, F. Proteome-wide structural probing of low-abundant protein interactions by crosslinking mass spectrometry. Anal Chem 92, 4016-4022 (2020).

212 Rozbesky, D., Rosulek, M., Kukacka, Z., Chmelik, J., Man, P. \& Novak, P. Impact of Chemical Cross-Linking on Protein Structure and Function. Anal Chem 90, 1104-1113 (2018).

213 Steigenberger, B., Pieters, R. J., Heck, A. J. R. \& Scheltema, R. A. PhoX: An IMAC-Enrichable Cross-Linking Reagent. ACS Cent Sci 5, 1514-1522 (2019).

214 Leitner, A., Reischl, R., Walzthoeni, T., Herzog, F., Bohn, S., Forster, F. \& Aebersold, R. Expanding the chemical cross-linking toolbox by the use of multiple proteases and enrichment by size exclusion chromatography. Mol Cell Proteomics 11, M111 014126 (2012).

215 Rinner, O., Seebacher, J., Walzthoeni, T., Mueller, L. N., Beck, M., Schmidt, A., Mueller, M. \& Aebersold, R. Identification of cross-linked peptides from large sequence databases. Nat Methods 5, 315-318 (2008).

216 Petrotchenko, E. V., Serpa, J. J. \& Borchers, C. H. An isotopically coded CIDcleavable biotinylated cross-linker for structural proteomics. Mol Cell Proteomics 10, M110 001420 (2011).

217 Kaake, R. M., Wang, X., Burke, A., Yu, C., Kandur, W., Yang, Y., Novtisky, E. J., Second, T., Duan, J., Kao, A., Guan, S., Vellucci, D., Rychnovsky, S. D. \& Huang, L. A new in vivo cross-linking mass spectrometry platform to define protein-protein interactions in living cells. Mol Cell Proteomics 13, 3533-3543 (2014).

218 Tan, D., Li, Q., Zhang, M.-J., Liu, C., Ma, C., Zhang, P., Ding, Y.-H., Fan, S.-B., Tao, L., Yang, B., Li, X., Ma, S., Liu, J., Feng, B., Liu, X., Wang, H.-W., He, S.-M., Gao, N., Ye, K., Dong, M.-Q. \& Lei, X. Trifunctional cross-linker for mapping protein-protein interaction networks and comparing protein conformational states. eLife 5 (2016).

219 Huang, R., Zhu, W., Wu, Y., Chen, J., Yu, J., Jiang, B., Chen, H. \& Chen, W. A novel mass spectrometry-cleavable, phosphate-based enrichable and multitargeting protein cross-linker. Chem Sci 10, 6443-6447 (2019).

220 Yilmaz, S., Shiferaw, G. A., Rayo, J., Economou, A., Martens, L. \& Vandermarliere, E. Cross-linked peptide identification: A computational forest of algorithms. Mass Spectrom Rev 37, 738-749 (2018).

221 Kao, A., Chiu, C. L., Vellucci, D., Yang, Y., Patel, V. R., Guan, S., Randall, A., Baldi, P., Rychnovsky, S. D. \& Huang, L. Development of a novel cross-linking strategy for fast and accurate identification of cross-linked peptides of protein complexes. Mol Cell Proteomics 10, M110 002212 (2011).

222 Muller, M. Q., Dreiocker, F., Ihling, C. H., Schafer, M. \& Sinz, A. Cleavable crosslinker for protein structure analysis: reliable identification of cross-linking products by tandem MS. Anal Chem 82, 6958-6968 (2010).

223 Tang, X. \& Bruce, J. E. A new cross-linking strategy: protein interaction reporter (PIR) technology for protein-protein interaction studies. Mol Biosyst 6, 939-947 (2010).

224 Tran, B. Q., Goodlett, D. R. \& Goo, Y. A. Advances in protein complex analysis by chemical cross-linking coupled with mass spectrometry (CXMS) and bioinformatics. Biochimica et Biophysica Acta (BBA) - Proteins and Proteomics 1864, 123-129 (2016). 
225 Chen, Z. A. \& Rappsilber, J. Protein Dynamics in Solution by Quantitative Crosslinking/Mass Spectrometry. Trends in Biochemical Sciences 43, 908-920 (2018).

226 Fischer, L., Chen, Z. A. \& Rappsilber, J. Quantitative cross-linking/mass spectrometry using isotope-labelled cross-linkers. J Proteomics 88, 120-128 (2013).

227 Schmidt, C. \& Robinson, C. V. A comparative cross-linking strategy to probe conformational changes in protein complexes. Nat Protoc 9, 2224-2236 (2014).

228 Tomko, Robert J., Taylor, David W., Chen, Zhuo A., Wang, H.-W., Rappsilber, J. \& Hochstrasser, M. A Single a Helix Drives Extensive Remodeling of the Proteasome Lid and Completion of Regulatory Particle Assembly. Cell 163, 432444 (2015).

229 Yu, C., Mao, H., Novitsky, E. J., Tang, X., Rychnovsky, S. D., Zheng, N. \& Huang, L. Gln40 deamidation blocks structural reconfiguration and activation of SCF ubiquitin ligase complex by Nedd8. Nature Communications 6 (2015).

230 Boelt, S. G., Norn, C., Rasmussen, M. I., André, I., Čiplys, E., Slibinskas, R., Houen, G. \& Højrup, P. Mapping the $\mathrm{Ca} 2+$ induced structural change in calreticulin. Journal of Proteomics 142, 138-148 (2016).

231 Chen, Z. A., Pellarin, R., Fischer, L., Sali, A., Nilges, M., Barlow, P. N. \& Rappsilber, J. Structure of Complement C3(H2O) Revealed By Quantitative Cross-Linking/Mass Spectrometry And Modeling. Mol Cell Proteomics 15, 27302743 (2016).

232 Chen, Z., Fischer, L., Tahir, S., Bukowski-Wills, J. C., Barlow, P. \& Rappsilber, J. Quantitative cross-linking/mass spectrometry reveals subtle protein conformational changes. Wellcome Open Res 1, 5 (2016).

233 Yu, C., Huszagh, A., Viner, R., Novitsky, E. J., Rychnovsky, S. D. \& Huang, L. Developing a Multiplexed Quantitative Cross-Linking Mass Spectrometry Platform for Comparative Structural Analysis of Protein Complexes. Anal Chem 88, 1030110308 (2016).

234 Muller, F., Fischer, L., Chen, Z. A., Auchynnikava, T. \& Rappsilber, J. On the Reproducibility of Label-Free Quantitative Cross-Linking/Mass Spectrometry. $J$ Am Soc Mass Spectrom 29, 405-412 (2018).

235 Muller, F., Kolbowski, L., Bernhardt, O. M., Reiter, L. \& Rappsilber, J. Dataindependent Acquisition Improves Quantitative Cross-linking Mass Spectrometry. Mol Cell Proteomics 18, 786-795 (2019).

236 Chen, Z. A. \& Rappsilber, J. Quantitative cross-linking/mass spectrometry to elucidate structural changes in proteins and their complexes. Nat Protoc 14, 171201 (2019).

237 Knop, M., Siegers, K., Pereira, G., Zachariae, W., Winsor, B., Nasmyth, K. \& Schiebel, E. Epitope tagging of yeast genes using a PCR-based strategy: more tags and improved practical routines. Yeast 15, 963-972 (1999).

238 Simon, M. \& Faye, G. Steps in processing of the mitochondrial cytochrome oxidase subunit I pre-mRNA affected by a nuclear mutation in yeast. Proceedings of the National Academy of Sciences 81, 8-12 (1984).

239 Meisinger, C., Pfanner, N. \& Truscott, K. N. Isolation of yeast mitochondria. Methods Mol Biol 313, 33-39 (2006).

240 Ryan, M. T., Voos, W. \& Pfanner, N. Assaying protein import into mitochondria. Methods Cell Biol 65, 189-215 (2001).

241 Bareth, B., Dennerlein, S., Mick, D. U., Nikolov, M., Urlaub, H. \& Rehling, P. The Heme a Synthase Cox15 Associates with Cytochrome c Oxidase Assembly Intermediates during Cox1 Maturation. Molecular and Cellular Biology 33, 41284137 (2013).

242 Schagger, H. \& von Jagow, G. Blue native electrophoresis for isolation of membrane protein complexes in enzymatically active form. Anal Biochem 199, 223-231 (1991). 
243 Schagger, H., Cramer, W. A. \& von Jagow, G. Analysis of molecular masses and oligomeric states of protein complexes by blue native electrophoresis and isolation of membrane protein complexes by two-dimensional native electrophoresis. Anal Biochem 217, 220-230 (1994).

244 Gallagher, S., Winston, S. E., Fuller, S. A. \& Hurrell, J. G. Immunoblotting and immunodetection. Curr Protoc Neurosci Chapter 5, Unit 519 (2004).

245 Lavdovskaia, E., Kolander, E., Steube, E., Mai, M. M.-Q., Urlaub, H. \& RichterDennerlein, R. The human Obg protein GTPBP10 is involved in mitoribosomal biogenesis. Nucleic Acids Research 46, 8471-8482 (2018).

246 Callegari, S., Richter, F., Chojnacka, K., Jans, D. C., Lorenzi, I., Pacheu-Grau, D., Jakobs, S., Lenz, C., Urlaub, H., Dudek, J., Chacinska, A. \& Rehling, P. TIM29 is a subunit of the human carrier translocase required for protein transport. FEBS Lett 590, 4147-4158 (2016).

247 Linden, A., Deckers, M., Parfentev, I., Pflanz, R., Homberg, B., Neumann, P., Ficner, R., Rehling, P. \& Urlaub, H. A cross-linking mass spectrometry approach defines protein interactions in yeast mitochondria. Molecular \& Cellular Proteomics 19, 1161-1178 (2020).

248 Kobashi, K. Catalytic oxidation of sulfhydryl groups by o-phenanthroline copper complex. Biochim Biophys Acta 158, 239-245 (1968).

249 Capelo, J. L., Carreira, R., Diniz, M., Fernandes, L., Galesio, M., Lodeiro, C., Santos, H. M. \& Vale, G. Overview on modern approaches to speed up protein identification workflows relying on enzymatic cleavage and mass spectrometrybased techniques. Analytica Chimica Acta 650, 151-159 (2009).

250 Laemmli, U. K. Cleavage of structural proteins during the assembly of the head of bacteriophage T4. Nature 227, 680-685 (1970).

251 Shevchenko, A., Tomas, H., Havlis, J., Olsen, J. V. \& Mann, M. In-gel digestion for mass spectrometric characterization of proteins and proteomes. Nat Protoc 1, 2856-2860 (2006).

252 Rappsilber, J., Ishihama, Y. \& Mann, M. Stop and go extraction tips for matrixassisted laser desorption/ionization, nanoelectrospray, and LC/MS sample pretreatment in proteomics. Anal Chem 75, 663-670 (2003).

253 Searle, B. C. Scaffold: a bioinformatic tool for validating MS/MS-based proteomic studies. Proteomics 10, 1265-1269 (2010).

254 Cox, J. \& Mann, M. MaxQuant enables high peptide identification rates, individualized p.p.b.-range mass accuracies and proteome-wide protein quantification. Nat Biotechnol 26, 1367-1372 (2008).

255 Yang, B., Wu, Y. J., Zhu, M., Fan, S. B., Lin, J., Zhang, K., Li, S., Chi, H., Li, Y. X., Chen, H. F., Luo, S. K., Ding, Y. H., Wang, L. H., Hao, Z., Xiu, L. Y., Chen, S., Ye, K., He, S. M. \& Dong, M. Q. Identification of cross-linked peptides from complex samples. Nat Methods 9, 904-906 (2012).

256 Fan, S. B., Meng, J. M., Lu, S., Zhang, K., Yang, H., Chi, H., Sun, R. X., Dong, M. Q. \& He, S. M. Using pLink to Analyze Cross-Linked Peptides. Curr Protoc Bioinformatics 49, 821 21-19 (2015).

257 Chen, Z. A., Fischer, L., Cox, J. \& Rappsilber, J. Quantitative Cross-linking/Mass Spectrometry Using Isotope-labeled Cross-linkers and MaxQuant. Mol Cell Proteomics 15, 2769-2778 (2016).

258 Chen, Z. L., Meng, J. M., Cao, Y., Yin, J. L., Fang, R. Q., Fan, S. B., Liu, C., Zeng, W. F., Ding, Y. H., Tan, D., Wu, L., Zhou, W. J., Chi, H., Sun, R. X., Dong, M. Q. \& He, S. M. A high-speed search engine pLink 2 with systematic evaluation for proteome-scale identification of cross-linked peptides. Nat Commun 10, 3404 (2019).

259 Alford, R. F., Koehler Leman, J., Weitzner, B. D., Duran, A. M., Tilley, D. C., Elazar, A. \& Gray, J. J. An Integrated Framework Advancing Membrane Protein Modeling and Design. PLoS Comput Biol 11, e1004398 (2015). 
260 Gray, J. J., Moughon, S., Wang, C., Schueler-Furman, O., Kuhlman, B., Rohl, C. A. \& Baker, D. Protein-protein docking with simultaneous optimization of rigidbody displacement and side-chain conformations. J Mol Biol 331, 281-299 (2003).

261 Chaudhury, S., Berrondo, M., Weitzner, B. D., Muthu, P., Bergman, H. \& Gray, J. $\mathrm{J}$. Benchmarking and analysis of protein docking performance in Rosetta v3.2. PLoS One 6, e22477 (2011).

262 Lomize, M. A., Pogozheva, I. D., Joo, H., Mosberg, H. I. \& Lomize, A. L. OPM database and PPM web server: resources for positioning of proteins in membranes. Nucleic Acids Res 40, D370-376 (2012).

263 Feng, Y., Li, W., Li, J., Wang, J., Ge, J., Xu, D., Liu, Y., Wu, K., Zeng, Q., Wu, J. W., Tian, C., Zhou, B. \& Yang, M. Structural insight into the type-II mitochondrial NADH dehydrogenases. Nature 491, 478-482 (2012).

264 Ruprecht, J. J., Hellawell, A. M., Harding, M., Crichton, P. G., McCoy, A. J. \& Kunji, E. R. Structures of yeast mitochondrial ADP/ATP carriers support a domain-based alternating-access transport mechanism. Proc Natl Acad Sci U S A 111, E426-434 (2014).

265 Barth, P., Wallner, B. \& Baker, D. Prediction of membrane protein structures with complex topologies using limited constraints. Proc Natl Acad Sci U S A 106, 1409-1414 (2009).

266 Yarov-Yarovoy, V., Schonbrun, J. \& Baker, D. Multipass membrane protein structure prediction using Rosetta. Proteins 62, 1010-1025 (2006).

267 Viklund, H. \& Elofsson, A. OCTOPUS: improving topology prediction by two-track ANN-based preference scores and an extended topological grammar. Bioinformatics 24, 1662-1668 (2008).

268 Emsley, P., Lohkamp, B., Scott, W. G. \& Cowtan, K. Features and development of Coot. Acta Crystallogr D Biol Crystallogr 66, 486-501 (2010).

269 Shannon, P., Markiel, A., Ozier, O., Baliga, N. S., Wang, J. T., Ramage, D., Amin, N., Schwikowski, B. \& Ideker, T. Cytoscape: a software environment for integrated models of biomolecular interaction networks. Genome Res 13, 24982504 (2003).

270 Combe, C. W., Fischer, L. \& Rappsilber, J. xiNET: cross-link network maps with residue resolution. Mol Cell Proteomics 14, 1137-1147 (2015).

271 Grimm, M., Zimniak, T., Kahraman, A. \& Herzog, F. xVis: a web server for the schematic visualization and interpretation of crosslink-derived spatial restraints. Nucleic Acids Res 43, W362-369 (2015).

272 Tyanova, S., Temu, T., Sinitcyn, P., Carlson, A., Hein, M. Y., Geiger, T., Mann, M. \& Cox, J. The Perseus computational platform for comprehensive analysis of (prote)omics data. Nat Methods 13, 731-740 (2016).

273 van Zundert, G. C. \& Bonvin, A. M. DisVis: quantifying and visualizing accessible interaction space of distance-restrained biomolecular complexes. Bioinformatics 31, 3222-3224 (2015).

274 Pettersen, E. F., Goddard, T. D., Huang, C. C., Couch, G. S., Greenblatt, D. M., Meng, E. C. \& Ferrin, T. E. UCSF Chimera--a visualization system for exploratory research and analysis. J Comput Chem 25, 1605-1612 (2004).

275 Kosinski, J., von Appen, A., Ori, A., Karius, K., Muller, C. W. \& Beck, M. Xlink Analyzer: software for analysis and visualization of cross-linking data in the context of three-dimensional structures. J Struct Biol 189, 177-183 (2015).

276 Yuan, C., Gao, J., Guo, J., Bai, L., Marshall, C., Cai, Z., Wang, L. \& Xiao, M. Dimethyl sulfoxide damages mitochondrial integrity and membrane potential in cultured astrocytes. PLoS One 9, e107447 (2014).

277 Kanehisa, M. \& Goto, S. KEGG: kyoto encyclopedia of genes and genomes. Nucleic Acids Res 28, 27-30 (2000). 
278 Yaffe, M. P., Jensen, R. E. \& Guido, E. C. The major 45-kDa protein of the yeast mitochondrial outer membrane is not essential for cell growth or mitochondrial function. J Biol Chem 264, 21091-21096 (1989).

279 Lauffer, S., Mabert, K., Czupalla, C., Pursche, T., Hoflack, B., Rodel, G. \& Krause-Buchholz, U. Saccharomyces cerevisiae porin pore forms complexes with mitochondrial outer membrane proteins Om14p and Om45p. J Biol Chem 287, 17447-17458 (2012).

280 Lawson, J. E., Behal, R. H. \& Reed, L. J. Disruption and mutagenesis of the Saccharomyces cerevisiae PDX1 gene encoding the protein $\mathrm{X}$ component of the pyruvate dehydrogenase complex. Biochemistry 30, 2834-2839 (1991).

281 Smith, R. C., Reed, V. D. \& Hill, W. E. Oxidation of Thiols by Copper(li). Phosphorus Sulfur 90, 147-154 (1994).

282 Wu, F. \& Minteer, S. Krebs cycle metabolon: structural evidence of substrate channeling revealed by cross-linking and mass spectrometry. Angew Chem Int Ed Engl 54, 1851-1854 (2015).

283 Kim, J. M., Yoshikawa, H. \& Shirahige, K. A member of the YER057c/yjgf/Uk114 family links isoleucine biosynthesis and intact mitochondria maintenance in Saccharomyces cerevisiae. Genes Cells 6, 507-517 (2001).

284 Meisinger, C., Brix, J., Model, K., Pfanner, N. \& Ryan, M. T. The preprotein translocase of the outer mitochondrial membrane: receptors and a general import pore. Cell Mol Life Sci 56, 817-824 (1999).

285 Koehler, C. M., Jarosch, E., Tokatlidis, K., Schmid, K., Schweyen, R. J. \& Schatz, G. Import of mitochondrial carriers mediated by essential proteins of the intermembrane space. Science 279, 369-373 (1998).

286 Koehler, C. M., Merchant, S., Oppliger, W., Schmid, K., Jarosch, E., Dolfini, L., Junne, T., Schatz, G. \& Tokatlidis, K. Tim9p, an essential partner subunit of Tim10p for the import of mitochondrial carrier proteins. EMBO J 17, 6477-6486 (1998).

287 Schneider, H. C., Westermann, B., Neupert, W. \& Brunner, M. The nucleotide exchange factor MGE exerts a key function in the ATP-dependent cycle of mtHsp70-Tim44 interaction driving mitochondrial protein import. EMBO J 15, 57965803 (1996).

288 Esnault, Y., Blondel, M. O., Deshaies, R. J., Scheckman, R. \& Kepes, F. The yeast SSS1 gene is essential for secretory protein translocation and encodes a conserved protein of the endoplasmic reticulum. EMBO J 12, 4083-4093 (1993).

289 Yofe, I., Weill, U., Meurer, M., Chuartzman, S., Zalckvar, E., Goldman, O., BenDor, S., Schutze, C., Wiedemann, N., Knop, M., Khmelinskii, A. \& Schuldiner, M. One library to make them all: streamlining the creation of yeast libraries via a SWAp-Tag strategy. Nat Methods 13, 371-378 (2016).

290 Rampelt, H., Bohnert, M., Zerbes, R. M., Horvath, S. E., Warscheid, B., Pfanner, N. \& van der Laan, M. Mic10, a Core Subunit of the Mitochondrial Contact Site and Cristae Organizing System, Interacts with the Dimeric F 1 F o -ATP Synthase. Journal of Molecular Biology 429, 1162-1170 (2017).

291 Thevelein, J. M. \& Hohmann, S. Trehalose synthase: guard to the gate of glycolysis in yeast? Trends Biochem Sci 20, 3-10 (1995).

292 Pernambuco, M. B., Winderickx, J., Crauwels, M., Griffioen, G., Mager, W. H. \& Thevelein, J. M. Glucose-triggered signalling in Saccharomyces cerevisiae: different requirements for sugar phosphorylation between cells grown on glucose and those grown on non-fermentable carbon sources. Microbiology 142 ( Pt 7), 1775-1782 (1996).

293 Slavov, N. \& Botstein, D. Coupling among growth rate response, metabolic cycle, and cell division cycle in yeast. Mol Biol Cell 22, 1997-2009 (2011).

294 Visser, W., van Spronsen, E. A., Nanninga, N., Pronk, J. T., Gijs Kuenen, J. \& van Dijken, J. P. Effects of growth conditions on mitochondrial morphology in Saccharomyces cerevisiae. Antonie Van Leeuwenhoek 67, 243-253 (1995). 
295 Dejean, L., Beauvoit, B., Guerin, B. \& Rigoulet, M. Growth of the yeast Saccharomyces cerevisiae on a non-fermentable substrate: control of energetic yield by the amount of mitochondria. Biochim Biophys Acta 1457, 45-56 (2000).

296 Kirchman, P. A., Kim, S., Lai, C. Y. \& Jazwinski, S. M. Interorganelle signaling is a determinant of longevity in Saccharomyces cerevisiae. Genetics 152, 179-190 (1999).

297 Woo, D. K. \& Poyton, R. O. The absence of a mitochondrial genome in rho0 yeast cells extends lifespan independently of retrograde regulation. Exp Gerontol 44, 390-397 (2009).

298 Kozlowski, L. P. Proteome-pl: proteome isoelectric point database. Nucleic Acids Res 45, D1112-D1116 (2017).

299 Richter, F., Dennerlein, S., Nikolov, M., Jans, D. C., Naumenko, N., Aich, A., MacVicar, T., Linden, A., Jakobs, S., Urlaub, H., Langer, T. \& Rehling, P. ROMO1 is a constituent of the human presequence translocase required for YME1L protease import. J Cell Biol 218, 598-614 (2019).

300 Richter-Dennerlein, R., Korwitz, A., Haag, M., Tatsuta, T., Dargazanli, S., Baker, M., Decker, T., Lamkemeyer, T., Rugarli, E. I. \& Langer, T. DNAJC19, a mitochondrial cochaperone associated with cardiomyopathy, forms a complex with prohibitins to regulate cardiolipin remodeling. Cell Metab 20, 158-171 (2014).

301 Meisinger, C., Sommer, T. \& Pfanner, N. Purification of Saccharomcyes cerevisiae mitochondria devoid of microsomal and cytosolic contaminations. Anal Biochem 287, 339-342 (2000).

302 Packer, L., Pollak, J. K., Munn, E. A. \& Greville, G. D. Effect of high sucrose concentrations on mitochondria: analysis of mitochondrial populations by densitygradient centrifugation after fixation with glutaraldehyde. J Bioenerg 2, 305-316 (1971).

303 Notman, R., Noro, M., O'Malley, B. \& Anwar, J. Molecular basis for dimethylsulfoxide (DMSO) action on lipid membranes. J Am Chem Soc 128, 13982-13983 (2006).

304 Jacob, S. W. \& de la Torre, J. C. Pharmacology of dimethyl sulfoxide in cardiac and CNS damage. Pharmacol Rep 61, 225-235 (2009).

305 Mannella, C. A. The 'ins' and 'outs' of mitochondrial membrane channels. Trends Biochem Sci 17, 315-320 (1992).

306 Benz, R. Permeation of hydrophilic solutes through mitochondrial outer membranes: review on mitochondrial porins. Biochim Biophys Acta 1197, 167196 (1994).

307 Hiller, S., Abramson, J., Mannella, C., Wagner, G. \& Zeth, K. The 3D structures of VDAC represent a native conformation. Trends Biochem Sci 35, 514-521 (2010).

308 Choudhary, O. P., Paz, A., Adelman, J. L., Colletier, J. P., Abramson, J. \& Grabe, M. Structure-guided simulations illuminate the mechanism of ATP transport through VDAC1. Nat Struct Mol Biol 21, 626-632 (2014).

309 Koulintchenko, M., Konstantinov, Y. \& Dietrich, A. Plant mitochondria actively import DNA via the permeability transition pore complex. EMBO J 22, 1245-1254 (2003).

310 Kruger, V., Becker, T., Becker, L., Montilla-Martinez, M., Ellenrieder, L., Vogtle, F. N., Meyer, H. E., Ryan, M. T., Wiedemann, N., Warscheid, B., Pfanner, N., Wagner, R. \& Meisinger, C. Identification of new channels by systematic analysis of the mitochondrial outer membrane. J Cell Biol 216, 3485-3495 (2017).

311 Klingenberg, M. Molecular aspects of the adenine nucleotide carrier from mitochondria. Arch Biochem Biophys 270, 1-14 (1989).

312 LêQuôc, K. \& LêQuôc, D. Involvement of the ADPATP carrier in calcium-induced perturbations of the mitochondrial inner membrane permeability: Importance of the orientation of the nucleotide binding site. Archives of Biochemistry and Biophysics 265, 249-257 (1988). 
313 Brustovetsky, N., Tropschug, M., Heimpel, S., Heidkämper, D. \& Klingenberg, M. A Large Ca2+-Dependent Channel Formed by Recombinant ADP/ATP Carrier fromNeurospora crassaResembles the Mitochondrial Permeability Transition Pore†. Biochemistry 41, 11804-11811 (2002).

314 Giorgio, V., von Stockum, S., Antoniel, M., Fabbro, A., Fogolari, F., Forte, M., Glick, G. D., Petronilli, V., Zoratti, M., Szabo, I., Lippe, G. \& Bernardi, P. Dimers of mitochondrial ATP synthase form the permeability transition pore. Proceedings of the National Academy of Sciences 110, 5887-5892 (2013).

315 Urbani, A., Giorgio, V., Carrer, A., Franchin, C., Arrigoni, G., Jiko, C., Abe, K., Maeda, S., Shinzawa-Itoh, K., Bogers, J. F. M., McMillan, D. G. G., Gerle, C., Szabo, I. \& Bernardi, P. Purified F-ATP synthase forms a Ca(2+)-dependent highconductance channel matching the mitochondrial permeability transition pore. Nat Commun 10, 4341 (2019).

316 Hunter, D. R., Haworth, R. A. \& Southard, J. H. Relationship between configuration, function, and permeability in calcium-treated mitochondria. J Biol Chem 251, 5069-5077 (1976).

317 Antonenko, Y. N., Kinnally, K. W. \& Tedeschi, H. Identification of anion and cation pathways in the inner mitochondrial membrane by patch clamping of mouse liver mitoplasts. J Membr Biol 124, 151-158 (1991).

318 Szabo, I. \& Zoratti, M. The mitochondrial megachannel is the permeability transition pore. J Bioenerg Biomembr 24, 111-117 (1992).

319 Kamei, Y., Koushi, M., Aoyama, Y. \& Asakai, R. The yeast mitochondrial permeability transition is regulated by reactive oxygen species, endogenous $\mathrm{Ca}(2+)$ and Cpr3, mediating cell death. Biochim Biophys Acta Bioenerg 1859, 1313-1326 (2018).

320 Gonzalez-Lozano, M. A., Koopmans, F., Sullivan, P. F., Protze, J., Krause, G., Verhage, M., Li, K. W., Liu, F. \& Smit, A. B. Stitching the synapse: Cross-linking mass spectrometry into resolving synaptic protein interactions. Sci Adv 6, eaax5783 (2020).

321 de Winde, J. H. \& Grivell, L. A. Global regulation of mitochondrial biogenesis in Saccharomyces cerevisiae. Prog Nucleic Acid Res Mol Biol 46, 51-91 (1993).

322 Vos, S. M., Farnung, L., Boehning, M., Wigge, C., Linden, A., Urlaub, H. \& Cramer, P. Structure of activated transcription complex Pol II-DSIF-PAF-SPT6. Nature 560, 607-612 (2018).

323 van der Bliek, A. M., Sedensky, M. M. \& Morgan, P. G. Cell Biology of the Mitochondrion. Genetics 207, 843-871 (2017).

324 Blondin, G. A. \& Green, D. E. The mechanism of mitochondrial swelling. Proc Natl Acad Sci U S A 58, 612-619 (1967).

325 Gleason, N. J., Vostrikov, V. V., Greathouse, D. V. \& Koeppe, R. E. Buried lysine, but not arginine, titrates and alters transmembrane helix tilt. Proceedings of the National Academy of Sciences 110, 1692-1695 (2013).

326 Schey, K. L., Grey, A. C. \& Nicklay, J. J. Mass spectrometry of membrane proteins: a focus on aquaporins. Biochemistry 52, 3807-3817 (2013).

327 Mendes, M. L., Fischer, L., Chen, Z. A., Barbon, M., O'Reilly, F. J., Giese, S. H., Bohlke-Schneider, M., Belsom, A., Dau, T., Combe, C. W., Graham, M., Eisele, M. R., Baumeister, W., Speck, C. \& Rappsilber, J. An integrated workflow for crosslinking mass spectrometry. Mol Syst Biol 15, e8994 (2019).

328 Grauslund, M. \& Ronnow, B. Carbon source-dependent transcriptional regulation of the mitochondrial glycerol-3-phosphate dehydrogenase gene, GUT2, from Saccharomyces cerevisiae. Can J Microbiol 46, 1096-1100 (2000).

329 Trumbly, R. J. Glucose repression in the yeast Saccharomyces cerevisiae. Mol Microbiol 6, 15-21 (1992).

330 Hatefi, Y. \& Rieske, J. S. in Oxidation and Phosphorylation Methods in Enzymology 225-231 (1967). 
331 Tisdale, H. D. in Oxidation and Phosphorylation Methods in Enzymology 213215 (1967).

332 Rathore, S., Berndtsson, J., Marin-Buera, L., Conrad, J., Carroni, M., Brzezinski, P. \& Ott, M. Cryo-EM structure of the yeast respiratory supercomplex. Nat Struct Mol Biol 26, 50-57 (2019).

333 Matus-Ortega, M. G., Cardenas-Monroy, C. A., Flores-Herrera, O., MendozaHernandez, G., Miranda, M., Gonzalez-Pedrajo, B., Vazquez-Meza, H. \& Pardo, J. P. New complexes containing the internal alternative NADH dehydrogenase (Ndi1) in mitochondria of Saccharomyces cerevisiae. Yeast 32, 629-641 (2015).

334 Grandier-Vazeille, X. \& Guérin, M. Separation by Blue Native and Colorless Native Polyacrylamide Gel Electrophoresis of the Oxidative Phosphorylation Complexes of Yeast Mitochondria Solubilized by Different Detergents: Specific Staining of the Different Complexes. Analytical Biochemistry 242, 248-254 (1996).

335 Wittig, I. \& Schagger, H. Supramolecular organization of ATP synthase and respiratory chain in mitochondrial membranes. Biochim Biophys Acta 1787, 672680 (2009).

336 Boumans, H., Grivell, L. A. \& Berden, J. A. The Respiratory Chain in Yeast Behaves as a Single Functional Unit. Journal of Biological Chemistry 273, 48724877 (1998).

337 O'Malley, K., Pratt, P., Robertson, J., Lilly, M. \& Douglas, M. G. Selection of the nuclear gene for the mitochondrial adenine nucleotide translocator by genetic complementation of the op1 mutation in yeast. J Biol Chem 257, 2097-2103 (1982).

338 Pfaff, E., Heldt, H. W. \& Klingenberg, M. Adenine nucleotide translocation of mitochondria. Kinetics of the adenine nucleotide exchange. Eur J Biochem 10, 484-493 (1969).

339 Pfaff, E. \& Klingenberg, M. Adenine nucleotide translocation of mitochondria. 1. Specificity and control. Eur J Biochem 6, 66-79 (1968).

340 Klingenberg, M. The ADP and ATP transport in mitochondria and its carrier. Biochim Biophys Acta 1778, 1978-2021 (2008).

341 Lawson, J. E., Gawaz, M., Klingenberg, M. \& Douglas, M. G. Structure-function studies of adenine nucleotide transport in mitochondria. I. Construction and genetic analysis of yeast mutants encoding the ADP/ATP carrier protein of mitochondria. J Biol Chem 265, 14195-14201 (1990).

342 Ogunbona, O. B., Baile, M. G. \& Claypool, S. M. Cardiomyopathy-associated mutation in the ADP/ATP carrier reveals translation-dependent regulation of cytochrome c oxidase activity. Mol Biol Cell 29, 1449-1464 (2018).

343 LaMarche, A. E., Abate, M. I., Chan, S. H. \& Trumpower, B. L. Isolation and characterization of COX12, the nuclear gene for a previously unrecognized subunit of Saccharomyces cerevisiae cytochrome c oxidase. J Biol Chem 267, 22473-22480 (1992).

344 Carr, H. S. \& Winge, D. R. Assembly of cytochrome c oxidase within the mitochondrion. Acc Chem Res 36, 309-316 (2003).

345 Herrmann, J. M. \& Funes, S. Biogenesis of cytochrome oxidase-sophisticated assembly lines in the mitochondrial inner membrane. Gene 354, 43-52 (2005).

346 Fontanesi, F., Clemente, P. \& Barrientos, A. Cox25 teams up with Mss51, Ssc1, and Cox14 to regulate mitochondrial cytochrome $c$ oxidase subunit 1 expression and assembly in Saccharomyces cerevisiae. J Biol Chem 286, 555-566 (2011).

347 Glerum, D. M., Koerner, T. J. \& Tzagoloff, A. Cloning and characterization of COX14, whose product is required for assembly of yeast cytochrome oxidase. $J$ Biol Chem 270, 15585-15590 (1995).

348 McStay, G. P., Su, C. H. \& Tzagoloff, A. Stabilization of Cox1p intermediates by the Cox14p-Coa3p complex. FEBS Lett 587, 943-949 (2013). 
349 Perez-Martinez, X., Broadley, S. A. \& Fox, T. D. Mss51p promotes mitochondrial Cox1p synthesis and interacts with newly synthesized Cox1p. EMBO J 22, 59515961 (2003).

350 Manthey, G. M. \& McEwen, J. E. The product of the nuclear gene PET309 is required for translation of mature mRNA and stability or production of introncontaining RNAs derived from the mitochondrial COX1 locus of Saccharomyces cerevisiae. EMBO J 14, $4031-4043$ (1995).

351 Fontanesi, F., Jin, C., Tzagoloff, A. \& Barrientos, A. Transcriptional activators HAP/NF-Y rescue a cytochrome c oxidase defect in yeast and human cells. Hum Mol Genet 17, 775-788 (2008).

352 Garlich, J., Strecker, V., Wittig, I. \& Stuart, R. A. Mutational Analysis of the QRRQ Motif in the Yeast Hig1 Type 2 Protein Rcf1 Reveals a Regulatory Role for the Cytochrome c Oxidase Complex. J Biol Chem 292, 5216-5226 (2017).

353 McEwen, J. E., Ko, C., Kloeckner-Gruissem, B. \& Poyton, R. O. Nuclear functions required for cytochrome c oxidase biogenesis in Saccharomyces cerevisiae.

Characterization of mutants in 34 complementation groups. J Biol Chem 261, 11872-11879 (1986).

354 Chacinska, A., Pfannschmidt, S., Wiedemann, N., Kozjak, V., Sanjuan Szklarz, L. K., Schulze-Specking, A., Truscott, K. N., Guiard, B., Meisinger, C. \& Pfanner, N. Essential role of Mia40 in import and assembly of mitochondrial intermembrane space proteins. EMBO J 23, 3735-3746 (2004).

355 Lu, H., Allen, S., Wardleworth, L., Savory, P. \& Tokatlidis, K. Functional TIM10 Chaperone Assembly Is Redox-regulatedin Vivo. Journal of Biological Chemistry 279, 18952-18958 (2004).

356 Sideris, D. P., Petrakis, N., Katrakili, N., Mikropoulou, D., Gallo, A., Ciofi-Baffoni, S., Banci, L., Bertini, I. \& Tokatlidis, K. A novel intermembrane space-targeting signal docks cysteines onto Mia40 during mitochondrial oxidative folding. The Journal of Cell Biology 187, 1007-1022 (2009).

357 van Dijken, J. P., van den Bosch, E., Hermans, J. J., de Miranda, L. R. \& Scheffers, W. A. Alcoholic fermentation by 'non-fermentative' yeasts. Yeast 2, 123-127 (1986).

358 Holzer, H. \& Goedde, H. W. [Two ways from pyruvate to acetyl-coenzyme A in yeast]. Biochem $Z$ 329, 175-191 (1957).

359 Ring, J., Sommer, C., Carmona-Gutierrez, D., Ruckenstuhl, C., Eisenberg, T. \& Madeo, F. The metabolism beyond programmed cell death in yeast. Exp Cell Res 318, 1193-1200 (2012).

360 Kresze, G. B. \& Ronft, H. Pyruvate dehydrogenase complex from baker's yeast. 1. Purification and some kinetic and regulatory properties. Eur J Biochem 119, 573-579 (1981).

361 Boiteux, A. \& Hess, B. Allosteric properties of yeast pyruvate decarboxylase. FEBS Lett 9, 293-296 (1970).

362 van Urk, H., Schipper, D., Breedveld, G. J., Mak, P. R., Scheffers, W. A. \& van Dijken, J. P. Localization and kinetics of pyruvate-metabolizing enzymes in relation to aerobic alcoholic fermentation in Saccharomyces cerevisiae CBS 8066 and Candida utilis CBS 621. Biochim Biophys Acta 992, 78-86 (1989).

363 Hohmann, S. Characterization of PDC6, a third structural gene for pyruvate decarboxylase in Saccharomyces cerevisiae. J Bacteriol 173, 7963-7969 (1991).

364 Lawson, J. E., Niu, X. D. \& Reed, L. J. Functional analysis of the domains of dihydrolipoamide acetyltransferase from Saccharomyces cerevisiae. Biochemistry 30, 11249-11254 (1991).

365 Miran, S. G., Lawson, J. E. \& Reed, L. J. Characterization of PDH beta 1, the structural gene for the pyruvate dehydrogenase beta subunit from Saccharomyces cerevisiae. Proc Natl Acad Sci U S A 90, 1252-1256 (1993).

366 Wenzel, T. J., Berg, M. A., Visser, W., Berg, J. A. \& Steensma, H. Y. Characterization of Saccharomyces cerevisiae mutants lacking the E1alpha 
subunit of the pyruvate dehydrogenase complex. European Journal of Biochemistry 209, 697-705 (1992).

367 Ronnow, B. \& Kielland-Brandt, M. C. GUT2, a gene for mitochondrial glycerol 3phosphate dehydrogenase of Saccharomyces cerevisiae. Yeast 9, 1121-1130 (1993).

368 Pavlik, P., Simon, M., Schuster, T. \& Ruis, H. The glycerol kinase (GUT1) gene of Saccharomyces cerevisiae: cloning and characterization. Curr Genet 24, 21-25 (1993).

369 Grauslund, M., Lopes, J. M. \& Ronnow, B. Expression of GUT1, which encodes glycerol kinase in Saccharomyces cerevisiae, is controlled by the positive regulators Adr1p, Ino2p and Ino4p and the negative regulator Opi1p in a carbon source-dependent fashion. Nucleic Acids Res 27, 4391-4398 (1999).

370 Pronk, J. T., Wenzel, T. J., Luttik, M. A. H., Klaassen, C. C. M., Scheffers, W. A., Steensma, H. Y. \& van Dijken, J. P. Energetic aspects of glucose metabolism in a pyruvate-dehydrogenase-negative mutant of Saccharomyces cerevisiae.

Microbiology 140, 601-610 (1994).

371 Boubekeur, S., Camougrand, N., Bunoust, O., Rigoulet, M. \& Guerin, B. Participation of acetaldehyde dehydrogenases in ethanol and pyruvate metabolism of the yeast Saccharomyces cerevisiae. Eur J Biochem 268, 50575065 (2001).

372 Dickinson, J. R., Roy, D. J. \& Dawes, I. W. A mutation affecting lipoamide dehydrogenase, pyruvate dehydrogenase and 2-oxoglutarate dehydrogenase activities in Saccharomyces cerevisiae. Mol Gen Genet 204, 103-107 (1986).

373 Repetto, B. \& Tzagoloff, A. In vivo assembly of yeast mitochondrial alphaketoglutarate dehydrogenase complex. Mol Cell Biol 11, 3931-3939 (1991).

374 DeRisi, J. L., lyer, V. R. \& Brown, P. O. Exploring the metabolic and genetic control of gene expression on a genomic scale. Science 278, 680-686 (1997).

375 Brauer, M. J., Saldanha, A. J., Dolinski, K. \& Botstein, D. Homeostatic adjustment and metabolic remodeling in glucose-limited yeast cultures. Mol Biol Cell 16, 2503-2517 (2005).

376 Roberts, G. G., 3rd \& Hudson, A. P. Rsf1p is required for an efficient metabolic shift from fermentative to glycerol-based respiratory growth in $\mathrm{S}$. cerevisiae. Yeast 26, 95-110 (2009).

377 Kulkarni, M. S. \& Sherman, F. NAT2, an essential gene encoding methionine N alpha-acetyltransferase in the yeast Saccharomyces cerevisiae. J Biol Chem 269, 13141-13147 (1994).

378 Van Damme, P., Hole, K., Pimenta-Marques, A., Helsens, K., Vandekerckhove, J., Martinho, R. G., Gevaert, K. \& Arnesen, T. NatF contributes to an evolutionary shift in protein N-terminal acetylation and is important for normal chromosome segregation. PLoS Genet 7, e1002169 (2011).

379 Lienhart, W. D., Gudipati, V. \& Macheroux, P. The human flavoproteome. Arch Biochem Biophys 535, 150-162 (2013).

380 Matouschek, A., Rospert, S., Schmid, K., Glick, B. S. \& Schatz, G. Cyclophilin catalyzes protein folding in yeast mitochondria. Proc Natl Acad Sci U S A 92, 6319-6323 (1995).

381 Stefely, J. A. \& Pagliarini, D. J. Biochemistry of Mitochondrial Coenzyme Q Biosynthesis. Trends Biochem Sci 42, 824-843 (2017).

382 Oxelmark, E., Marchini, A., Malanchi, I., Magherini, F., Jaquet, L., Hajibagheri, M. A., Blight, K. J., Jauniaux, J. C. \& Tommasino, M. Mmf1p, a novel yeast mitochondrial protein conserved throughout evolution and involved in maintenance of the mitochondrial genome. Mol Cell Biol 20, 7784-7797 (2000).

383 Bauer, B. E., Wolfger, H. \& Kuchler, K. Inventory and function of yeast ABC proteins: about sex, stress, pleiotropic drug and heavy metal resistance. Biochim Biophys Acta 1461, 217-236 (1999). 
384 Kehrein, K., Schilling, R., Moller-Hergt, B. V., Wurm, C. A., Jakobs, S., Lamkemeyer, T., Langer, T. \& Ott, M. Organization of Mitochondrial Gene Expression in Two Distinct Ribosome-Containing Assemblies. Cell Rep 10, 843853 (2015).

385 Bukau, B. \& Horwich, A. L. The Hsp70 and Hsp60 chaperone machines. Cell 92, 351-366 (1998).

386 Jagow, G. \& Klingenberg, M. Pathways of Hydrogen in Mitochondria of Saccharomyces carlsbergensis. European Journal of Biochemistry 12, 583-592 (1970).

387 Rapaport, D. The mitochondrial protein OM45 is exposed to the cytosol. J Biol Chem 287, 27415; author reply 27416 (2012).

388 Lauffer, S., Rödel, G. \& Krause-Buchholz, U. Reply to Rapaport: The Mitochondrial Protein OM45 Is Exposed to the Cytosol. Journal of Biological Chemistry 287, 27416-27416 (2012).

389 Riezman, H., Hay, R., Gasser, S., Daum, G., Schneider, G., Witte, C. \& Schatz, G. The outer membrane of yeast mitochondria: isolation of outside-out sealed vesicles. EMBO J 2, 1105-1111 (1983).

390 Jensen, R. E. \& Sesaki, H. UGO1 Encodes an Outer Membrane Protein Required for Mitochondrial Fusion. Journal of Cell Biology 152, 1123-1134 (2001).

391 Wenz, L. S., Opalinski, L., Schuler, M. H., Ellenrieder, L., leva, R., Bottinger, L., Qiu, J., van der Laan, M., Wiedemann, N., Guiard, B., Pfanner, N. \& Becker, T. The presequence pathway is involved in protein sorting to the mitochondrial outer membrane. EMBO Rep 15, 678-685 (2014).

392 Song, J., Tamura, Y., Yoshihisa, T. \& Endo, T. A novel import route for an Nanchor mitochondrial outer membrane protein aided by the TIM23 complex. EMBO Rep 15, 670-677 (2014).

393 Overkamp, K. M., Bakker, B. M., Kötter, P., van Tuijl, A., de Vries, S., van Dijken, J. P. \& Pronk, J. T. In Vivo Analysis of the Mechanisms for Oxidation of Cytosolic NADH by Saccharomyces cerevisiaeMitochondria. Journal of Bacteriology 182, 2823-2830 (2000).

394 Lee, A. C., Xu, X., Blachly-Dyson, E., Forte, M. \& Colombini, M. The Role of Yeast VDAC Genes on the Permeability of the Mitochondrial Outer Membrane. Journal of Membrane Biology 161, 173-181 (1998).

395 Nunnari, J., Weissman, J. S., Schuldiner, M., Westermann, B., Lackner, L. L., DeVay, R. M., Hummel, E., Cassidy-Stone, A., Collins, S. R. \& Hoppins, S. A mitochondrial-focused genetic interaction map reveals a scaffold-like complex required for inner membrane organization in mitochondria. Journal of Cell Biology 195, 323-340 (2011).

396 Alkhaja, A. K., Jans, D. C., Nikolov, M., Vukotic, M., Lytovchenko, O., Ludewig, F., Schliebs, W., Riedel, D., Urlaub, H., Jakobs, S., Deckers, M. \& Fox, T. D. MINOS1 is a conserved component of mitofilin complexes and required for mitochondrial function and cristae organization. Molecular Biology of the Cell 23, 247-257 (2012).

397 Blum, T. B., Hahn, A., Meier, T., Davies, K. M. \& Kühlbrandt, W. Dimers of mitochondrial ATP synthase induce membrane curvature and self-assemble into rows. Proceedings of the National Academy of Sciences 116, 4250-4255 (2019).

398 Arselin, G., Vaillier, J., Salin, B., Schaeffer, J., Giraud, M. F., Dautant, A., Brethes, D. \& Velours, J. The modulation in subunits e and $g$ amounts of yeast ATP synthase modifies mitochondrial cristae morphology. J Biol Chem 279, 40392-40399 (2004).

399 Minauro-Sanmiguel, F., Wilkens, S. \& Garcia, J. J. Structure of dimeric mitochondrial ATP synthase: novel F0 bridging features and the structural basis of mitochondrial cristae biogenesis. Proc Natl Acad Sci U S A 102, 12356-12358 (2005). 
400 Cabezon, E., Arechaga, I., Jonathan, P., Butler, G. \& Walker, J. E. Dimerization of bovine F1-ATPase by binding the inhibitor protein, IF1. J Biol Chem 275, 28353-28355 (2000).

401 Dienhart, M., Pfeiffer, K., Schagger, H. \& Stuart, R. A. Formation of the yeast F1F0-ATP synthase dimeric complex does not require the ATPase inhibitor protein, Inh1. J Biol Chem 277, 39289-39295 (2002).

402 Pfanner, N., van der Laan, M., Amati, P., Capaldi, R. A., Caudy, A. A., Chacinska, A., Darshi, M., Deckers, M., Hoppins, S., Icho, T., Jakobs, S., Ji, J., Kozjak-Pavlovic, V., Meisinger, C., Odgren, P. R., Park, S. K., Rehling, P., Reichert, A. S., Sheikh, M. S., Taylor, S. S., Tsuchida, N., van der Bliek, A. M., van der Klei, I. J., Weissman, J. S., Westermann, B., Zha, J., Neupert, W. \& Nunnari, J. Uniform nomenclature for the mitochondrial contact site and cristae organizing system. The Journal of Cell Biology 204, 1083-1086 (2014).

403 Ujwal, R., Cascio, D., Colletier, J. P., Faham, S., Zhang, J., Toro, L., Ping, P. \& Abramson, J. The crystal structure of mouse VDAC1 at 2.3 A resolution reveals mechanistic insights into metabolite gating. Proc Natl Acad Sci U S A 105, 17742-17747 (2008).

404 Stanley, S., Dias, J. A., D'Arcangelis, D. \& Mannella, C. A. Peptide-specific antibodies as probes of the topography of the voltage-gated channel in the mitochondrial outer membrane of Neurospora crassa. J Biol Chem 270, 1669416700 (1995).

405 De Pinto, V., Prezioso, G., Thinnes, F., Link, T. A. \& Palmieri, F. Peptide-specific antibodies and proteases as probes of the transmembrane topology of the bovine heart mitochondrial porin. Biochemistry 30, 10191-10200 (1991).

406 Engelhardt, H., Meins, T., Poynor, M., Adams, V., Nussberger, S., Welte, W. \& Zeth, K. High-level expression, refolding and probing the natural fold of the human voltage-dependent anion channel isoforms I and II. J Membr Biol 216, 93105 (2007).

407 Demarquoy, J. \& Le Borgne, F. Crosstalk between mitochondria and peroxisomes. World J Biol Chem 6, 301-309 (2015).

408 Kalies, K. U., Görlich, D. \& Rapoport, T. A. Binding of ribosomes to the rough endoplasmic reticulum mediated by the Sec61p-complex. The Journal of Cell Biology 126, 925-934 (1994).

409 Corsi, A. K. \& Schekman, R. Mechanism of polypeptide translocation into the endoplasmic reticulum. J Biol Chem 271, 30299-30302 (1996).

410 Kornmann, B., Currie, E., Collins, S. R., Schuldiner, M., Nunnari, J., Weissman, J. S. \& Walter, P. An ER-mitochondria tethering complex revealed by a synthetic biology screen. Science 325, 477-481 (2009).

411 Liu, F., Lossl, P., Scheltema, R., Viner, R. \& Heck, A. J. R. Optimized fragmentation schemes and data analysis strategies for proteome-wide cross-link identification. Nat Commun 8, 15473 (2017).

412 Giese, S. H., Fischer, L. \& Rappsilber, J. A Study into the Collision-induced Dissociation (CID) Behavior of Cross-Linked Peptides. Mol Cell Proteomics 15, 1094-1104 (2016).

413 Fischer, L. \& Rappsilber, J. Quirks of Error Estimation in Cross-Linking/Mass Spectrometry. Analytical Chemistry 89, 3829-3833 (2017).

414 Zara, V., Conte, L. \& Trumpower, B. L. Identification and characterization of cytochrome bc(1) subcomplexes in mitochondria from yeast with single and double deletions of genes encoding cytochrome bc(1) subunits. FEBS J 274, 4526-4539 (2007).

415 Hägerhäll, C. Succinate: quinone oxidoreductases. Biochimica et Biophysica Acta (BBA) - Bioenergetics 1320, 107-141 (1997).

416 Elzinga, S. D., Bednarz, A. L., van Oosterum, K., Dekker, P. J. \& Grivell, L. A. Yeast mitochondrial NAD(+)-dependent isocitrate dehydrogenase is an RNAbinding protein. Nucleic Acids Res 21, 5328-5331 (1993). 
417 MacAlpine, D. M., Perlman, P. S. \& Butow, R. A. The high mobility group protein Abf2p influences the level of yeast mitochondrial DNA recombination intermediates in vivo. Proc Natl Acad Sci U S A 95, 6739-6743 (1998).

418 Hutu, D. P., Guiard, B., Chacinska, A., Becker, D., Pfanner, N., Rehling, P., van der Laan, M. \& Glick, B. Mitochondrial Protein Import Motor: Differential Role of Tim44 in the Recruitment of Pam17 and J-Complex to the Presequence Translocase. Molecular Biology of the Cell 19, 2642-2649 (2008).

419 Vial, S., Lu, H., Allen, S., Savory, P., Thornton, D., Sheehan, J. \& Tokatlidis, K. Assembly of Tim9 and Tim10 into a functional chaperone. J Biol Chem 277, 36100-36108 (2002).

420 Sakaue, H., Shiota, T., Ishizaka, N., Kawano, S., Tamura, Y., Tan, K. S., Imai, K., Motono, C., Hirokawa, T., Taki, K., Miyata, N., Kuge, O., Lithgow, T. \& Endo, T. Porin Associates with Tom22 to Regulate the Mitochondrial Protein Gate Assembly. Molecular Cell 73, 1044-1055.e1048 (2019).

421 Ellenrieder, L., Dieterle, M. P., Doan, K. N., Mårtensson, C. U., Floerchinger, A., Campo, M. L., Pfanner, N. \& Becker, T. Dual Role of Mitochondrial Porin in Metabolite Transport across the Outer Membrane and Protein Transfer to the Inner Membrane. Molecular Cell 73, 1056-1065.e1057 (2019).

422 Shiota, T., Imai, K., Qiu, J., Hewitt, V. L., Tan, K., Shen, H. H., Sakiyama, N., Fukasawa, Y., Hayat, S., Kamiya, M., Elofsson, A., Tomii, K., Horton, P., Wiedemann, N., Pfanner, N., Lithgow, T. \& Endo, T. Molecular architecture of the active mitochondrial protein gate. Science 349, 1544-1548 (2015).

423 Bausewein, T., Mills, D. J., Langer, J. D., Nitschke, B., Nussberger, S. \& Kühlbrandt, W. Cryo-EM Structure of the TOM Core Complex from Neurospora crassa. Cell 170, 693-700.e697 (2017).

424 Hiller, S., Garces, R. G., Malia, T. J., Orekhov, V. Y., Colombini, M. \& Wagner, G. Solution structure of the integral human membrane protein VDAC-1 in detergent micelles. Science 321, 1206-1210 (2008).

425 Bayrhuber, M., Meins, T., Habeck, M., Becker, S., Giller, K., Villinger, S., Vonrhein, C., Griesinger, C., Zweckstetter, M. \& Zeth, K. Structure of the human voltage-dependent anion channel. Proc Natl Acad Sci U S A 105, 15370-15375 (2008).

426 Dihanich, M., Suda, K. \& Schatz, G. A yeast mutant lacking mitochondrial porin is respiratory-deficient, but can recover respiration with simultaneous accumulation of an 86-kd extramitochondrial protein. EMBO J 6, 723-728 (1987).

427 Colombini, M. The published 3D structure of the VDAC channel: native or not? Trends in Biochemical Sciences 34, 382-389 (2009).

428 Colombini, M. VDAC structure, selectivity, and dynamics. Biochimica et Biophysica Acta (BBA) - Biomembranes 1818, 1457-1465 (2012).

429 Chavez, J. D., Schweppe, D. K., Eng, J. K. \& Bruce, J. E. In Vivo Conformational Dynamics of Hsp90 and Its Interactors. Cell Chem Biol 23, 716-726 (2016).

430 Chavez, J. D., Keller, A., Zhou, B., Tian, R. \& Bruce, J. E. Cellular Interactome Dynamics during Paclitaxel Treatment. Cell Rep 29, 2371-2383 e2375 (2019).

431 de Godoy, L. M. SILAC yeast: from labeling to comprehensive proteome quantification. Methods Mol Biol 1156, 81-109 (2014).

432 Brenes, A., Hukelmann, J., Bensaddek, D. \& Lamond, A. I. Multibatch TMT Reveals False Positives, Batch Effects and Missing Values. Mol Cell Proteomics 18, 1967-1980 (2019).

433 Kim, S. J., Fernandez-Martinez, J., Nudelman, I., Shi, Y., Zhang, W., Raveh, B., Herricks, T., Slaughter, B. D., Hogan, J. A., Upla, P., Chemmama, I. E., Pellarin, R., Echeverria, I., Shivaraju, M., Chaudhury, A. S., Wang, J., Williams, R., Unruh, J. R., Greenberg, C. H., Jacobs, E. Y., Yu, Z., de la Cruz, M. J., Mironska, R., Stokes, D. L., Aitchison, J. D., Jarrold, M. F., Gerton, J. L., Ludtke, S. J., Akey, C. W., Chait, B. T., Sali, A. \& Rout, M. P. Integrative structure and functional anatomy of a nuclear pore complex. Nature 555, 475-482 (2018). 
434 Gutierrez, C., Chemmama, I. E., Mao, H., Yu, C., Echeverria, I., Block, S. A., Rychnovsky, S. D., Zheng, N., Sali, A. \& Huang, L. Structural dynamics of the human COP9 signalosome revealed by cross-linking mass spectrometry and integrative modeling. Proceedings of the National Academy of Sciences 117, 4088-4098 (2020).

435 Calvo, S. E., Clauser, K. R. \& Mootha, V. K. MitoCarta2.0: an updated inventory of mammalian mitochondrial proteins. Nucleic Acids Res 44, D1251-1257 (2016).

436 Yu, C. \& Huang, L. Cross-Linking Mass Spectrometry: An Emerging Technology for Interactomics and Structural Biology. Anal Chem 90, 144-165 (2018).

437 Horovitz, A., Serrano, L., Avron, B., Bycroft, M. \& Fersht, A. R. Strength and cooperativity of contributions of surface salt bridges to protein stability. Journal of Molecular Biology 216, 1031-1044 (1990).

438 Nawrocki, G., Karaboga, A., Sugita, Y. \& Feig, M. Effect of protein-protein interactions and solvent viscosity on the rotational diffusion of proteins in crowded environments. Physical Chemistry Chemical Physics 21, 876-883 (2019).

439 Keller, A., Chavez, J. D., Felt, K. C. \& Bruce, J. E. Prediction of an Upper Limit for the Fraction of Interprotein Cross-Links in Large-Scale In Vivo Cross-Linking Studies. J Proteome Res 18, 3077-3085 (2019).

440 Posakony, J. W., England, J. M. \& Attardi, G. Mitochondrial growth and division during the cell cycle in HeLa cells. J Cell Biol 74, 468-491 (1977).

441 Grimes, G. W., Mahler, H. R. \& Perlman, R. S. Nuclear gene dosage effects on mitochondrial mass and DNA. J Cell Biol 61, 565-574 (1974).

442 Schwerzmann, K., Cruz-Orive, L. M., Eggman, R., Sanger, A. \& Weibel, E. R. Molecular architecture of the inner membrane of mitochondria from rat liver: a combined biochemical and stereological study. J Cell Biol 102, 97-103 (1986).

443 Gromiha, M. M. in Protein Bioinformatics 29-62 (2010).

444 Moser, C. C., Farid, T. A., Chobot, S. E. \& Dutton, P. L. Electron tunneling chains of mitochondria. Biochimica et Biophysica Acta (BBA) - Bioenergetics 1757, 1096-1109 (2006).

445 Fiedorczuk, K., Letts, J. A., Degliesposti, G., Kaszuba, K., Skehel, M. \& Sazanov, L. A. Atomic structure of the entire mammalian mitochondrial complex I. Nature 538, 406-410 (2016).

446 Vogel, R. O., Smeitink, J. A. M. \& Nijtmans, L. G. J. Human mitochondrial complex I assembly: A dynamic and versatile process. Biochimica et Biophysica Acta (BBA) - Bioenergetics 1767, 1215-1227 (2007).

447 Formosa, L. E., Dibley, M. G., Stroud, D. A. \& Ryan, M. T. Building a complex complex: Assembly of mitochondrial respiratory chain complex I. Seminars in Cell \& Developmental Biology 76, 154-162 (2018).

448 Guarani, V., Paulo, J., Zhai, B., Huttlin, E. L., Gygi, S. P. \& Harper, J. W. TIMMDC1/C3orf1 Functions as a Membrane-Embedded Mitochondrial Complex I Assembly Factor through Association with the MCIA Complex. Molecular and Cellular Biology 34, 847-861 (2014).

449 Andrews, B., Carroll, J., Ding, S., Fearnley, I. M. \& Walker, J. E. Assembly factors for the membrane arm of human complex I. Proceedings of the National Academy of Sciences 110, 18934-18939 (2013).

450 Stroud, D. A., Surgenor, E. E., Formosa, L. E., Reljic, B., Frazier, A. E., Dibley, M. G., Osellame, L. D., Stait, T., Beilharz, T. H., Thorburn, D. R., Salim, A. \& Ryan, M. T. Accessory subunits are integral for assembly and function of human mitochondrial complex I. Nature 538, 123-126 (2016).

451 Nijtmans, L. G., de Jong, L., Artal Sanz, M., Coates, P. J., Berden, J. A., Back, J. W., Muijsers, A. O., van der Spek, H. \& Grivell, L. A. Prohibitins act as a membrane-bound chaperone for the stabilization of mitochondrial proteins. $E M B O J$ J 19, 2444-2451 (2000). 
452 Berger, K. H. \& Yaffe, M. P. Prohibitin family members interact genetically with mitochondrial inheritance components in Saccharomyces cerevisiae. Mol Cell Biol 18, 4043-4052 (1998).

453 Tatsuta, T., Model, K. \& Langer, T. Formation of Membrane-bound Ring Complexes by Prohibitins in Mitochondria. Molecular Biology of the Cell 16, 248259 (2005).

454 Shetty, D. K., Kalamkar, K. P. \& Inamdar, M. S. OCIAD1 Controls Electron Transport Chain Complex I Activity to Regulate Energy Metabolism in Human Pluripotent Stem Cells. Stem Cell Reports 11, 128-141 (2018).

455 Antonicka, H., Lin, Z.-Y., Janer, A., Weraarpachai, W., Gingras, A.-C. \& Shoubridge, E. A. A high-density human mitochondrial proximity interaction network. bioRxiv (2020). https://doi.org/10.1101/2020.04.01.020479.

456 Fritzsche, R., Ihling, C. H., Gotze, M. \& Sinz, A. Optimizing the enrichment of cross-linked products for mass spectrometric protein analysis. Rapid Commun Mass Spectrom 26, 653-658 (2012).

457 Schmidt, R. \& Sinz, A. Improved single-step enrichment methods of cross-linked products for protein structure analysis and protein interaction mapping. Anal Bioanal Chem 409, 2393-2400 (2017).

458 Tinnefeld, V., Venne, A. S., Sickmann, A. \& Zahedi, R. P. Enrichment of CrossLinked Peptides Using Charge-Based Fractional Diagonal Chromatography (ChaFRADIC). J Proteome Res 16, 459-469 (2017).

459 Stadlmeier, M., Runtsch, L. S., Streshnev, F., Wuhr, M. \& Carell, T. A ClickChemistry-Based Enrichable Crosslinker for Structural and Protein Interaction Analysis by Mass Spectrometry. Chembiochem 21, 103-107 (2020).

460 Lu, L., Millikin, R. J., Solntsev, S. K., Rolfs, Z., Scalf, M., Shortreed, M. R. \& Smith, L. M. Identification of MS-Cleavable and Noncleavable Chemically CrossLinked Peptides with MetaMorpheus. J Proteome Res 17, 2370-2376 (2018).

461 Liu, F., Rijkers, D. T., Post, H. \& Heck, A. J. Proteome-wide profiling of protein assemblies by cross-linking mass spectrometry. Nat Methods 12, 1179-1184 (2015).

462 Gotze, M., Pettelkau, J., Fritzsche, R., Ihling, C. H., Schafer, M. \& Sinz, A. Automated assignment of MS/MS cleavable cross-links in protein 3D-structure analysis. J Am Soc Mass Spectrom 26, 83-97 (2015).

463 Parfentev, I., Schilbach, S., Cramer, P. \& Urlaub, H. An experimentally generated peptide database increases the sensitivity of XL-MS with complex samples. $J$ Proteomics 220, 103754 (2020).

464 Lima, D. B., de Lima, T. B., Balbuena, T. S., Neves-Ferreira, A. G. C., Barbosa, V. C., Gozzo, F. C. \& Carvalho, P. C. SIM-XL: A powerful and user-friendly tool for peptide cross-linking analysis. J Proteomics 129, 51-55 (2015).

465 Buncherd, H., Roseboom, W., Ghavim, B., Du, W., de Koning, L. J., de Koster, C. G. \& de Jong, L. Isolation of cross-linked peptides by diagonal strong cation exchange chromatography for protein complex topology studies by peptide fragment fingerprinting from large sequence databases. J Chromatogr $A$ 1348, 34-46 (2014).

466 Béganton, B., Solassol, I., Mangé, A. \& Solassol, J. Protein interactions study through proximity-labeling. Expert Review of Proteomics 16, 717-726 (2019).

467 Chen, C. L., Hu, Y., Udeshi, N. D., Lau, T. Y., Wirtz-Peitz, F., He, L., Ting, A. Y., Carr, S. A. \& Perrimon, N. Proteomic mapping in live Drosophila tissues using an engineered ascorbate peroxidase. Proc Natl Acad Sci U S A 112, 12093-12098 (2015).

468 Wessels, H. J., Vogel, R. O., van den Heuvel, L., Smeitink, J. A., Rodenburg, R. J., Nijtmans, L. G. \& Farhoud, M. H. LC-MS/MS as an alternative for SDS-PAGE in blue native analysis of protein complexes. Proteomics 9, 4221-4228 (2009).

469 Wessels, H. J., Vogel, R. O., Lightowlers, R. N., Spelbrink, J. N., Rodenburg, R. J., van den Heuvel, L. P., van Gool, A. J., Gloerich, J., Smeitink, J. A. \& Nijtmans, 
L. G. Analysis of 953 human proteins from a mitochondrial HEK293 fraction by complexome profiling. PLoS One 8, e68340 (2013).

470 Heide, H., Bleier, L., Steger, M., Ackermann, J., Drose, S., Schwamb, B., Zornig, M., Reichert, A. S., Koch, I., Wittig, I. \& Brandt, U. Complexome profiling identifies TMEM126B as a component of the mitochondrial complex I assembly complex. Cell Metab 16, 538-549 (2012).

471 Andersen, J. S., Wilkinson, C. J., Mayor, T., Mortensen, P., Nigg, E. A. \& Mann, M. Proteomic characterization of the human centrosome by protein correlation profiling. Nature 426, 570-574 (2003).

472 Rosenfeld, J., Capdevielle, J., Guillemot, J. C. \& Ferrara, P. In-gel digestion of proteins for internal sequence analysis after one- or two-dimensional gel electrophoresis. Anal Biochem 203, 173-179 (1992).

473 Heusel, M., Bludau, I., Rosenberger, G., Hafen, R., Frank, M., Banaei-Esfahani, A., van Drogen, A., Collins, B. C., Gstaiger, M. \& Aebersold, R. Complex-centric proteome profiling by SEC-SWATH-MS. Mol Syst Biol 15, e8438 (2019). 


\section{Acknowledgements}

Science is always teamwork. A lot of people supported me to finally give this work a common thread. Therefore, I would like to thank all friends and colleagues.

First, I thank Prof. Dr. Henning Urlaub who recognized something in me at the 'Advanced Proteomics' summer school in Brixen, 2016, and gave me the opportunity to graduate as part of his group. I remember when we discussed my first side project and he explained to me how to precipitate proteins, because I was ignorant and never did it before. I am still ignorant, but with a little bit more knowledge about cross-linking mass spectrometry - and protein precipitation.

I would like to thank Prof. Dr. Peter Rehling for his patience with this project that started long time ago. I was always astonished by his passion for science that regularly leaped over to me in numerous meetings with him, especially in challenging times.

I thank Prof. Dr. Jürgen Wienands and Dr. Alexander Stein for being part of my thesis advisory committee and for fruitful discussions about experiments and further plans, regardless of the project. Professor Wienands, I am sorry that not a single word about Bcells made it to this thesis as it was initially planned. Thanks to Dr. Ricarda RichterDennerlein and Prof. Dr. Stefan Jakobs for being members of my extended committee.

Special thanks go to Dr. Markus Deckers and Bettina Homberg for explaining to me the wonderful world of yeast mitochondria - theoretically and at the bench - in countless meetings and emails. The same holds true for Dr. Ricarda Richter-Dennerlein and Elena Lavdovskaia for their support with human mitochondria.

I am grateful for being part of the GGNB program "Biomolecules: Structure - Function Dynamics". It provides a supportive framework for all its students. It was always fun - also outside of the GGNB - with all the fellow PhD candidates, some of them who I can call friends nowadays.

I am very thankful for all the people of the Bioanalytical Mass Spectrometry Group, especially Monika Raabe, Uwe Pleßmann, Dr. Ralf Pflanz and Dr. Sabine König who made the daily work with mass spectrometers and organizations of side projects a lot more endurable. I want to thank Dr. Chung-Tien Lee who generated a lot of the data sets. Rest in peace. 
A special "thank you" goes to Dr. Iwan Parfentev who not only was a perfect scientific guide from the beginning to the very end of my path to the doctoral degree but who also listened to my problems apart from science when it was needed - and to techno music.

I am grateful that Dr. Iwan Parfentev, Dr. Andreia Cepeda, Bettina Homberg and Dr. Martijn Stax proofread parts of my thesis and that Hanna llse gave valuable advice for making high-quality figures. You really improved this work!

I would like to thank Aleksandar Chernev and Dr. Kuan-Ting Pan for sharing their almost endless knowledge about mass spectrometry and Dr. Ralf Pflanz for supporting me in wrapping up the results. I would like to thank Iwan, Aleks, Momchil, Alex, Fanni, Ralf, Ivan, Sofia, Pan, Yanlong, Kuan-Ting, Andreia, Luisa, Juliane, Christof, Uwe, Moni, Sabine, Selay, Christian, Olex, Sunit and Alexandra for a nice and fruitful working environment.

I am thankful for my flat mates Nadine, Hanna, Martijn and Marco who supported me in the last year of my $\mathrm{PhD}$ and especially in the last weeks of this journey, in which I was certainly not always the most pleasant flat mate.

I will also thank my friends at home, the "Jote Jungens" Thomas, Thomas, Mario, Pascal, Marc, Stefan and Maurice, Jenny and Ben, Sarah, Marc, my former fellow students Christine, Thuva, Pascal, Marius and Aylin, Nadine and Britta, my conference buddy Carolin, Daniel, Eric and Lukas, and Patricia and Jan-Dieter for remembering me that there is still a life apart from science.

And, most importantly, I thank my parents, for their unconditional love and support. Always. 